\title{
Detector Development for the High Luminosity Large Hadron Collider
}

\author{
Dissertation \\ zur Erlangung des mathematisch-naturwissenschaftlichen Doktorgrades \\ „Doctor rerum naturalium“ \\ der Georg-August-Universität Göttingen \\ im Promotionsprogramm ProPhys \\ der Georg-August University School of Science (GAUSS)
}

\author{
vorgelegt von \\ Julia Rieger \\ aus Münden
}

Göttingen, 2016 
Betreuungsausschuss

Prof. Dr. Arnulf Quadt

II. Physikalisches Institut, Georg-August-Universität Göttingen

PD Dr. Jörn Große-Knetter

II. Physikalisches Institut, Georg-August-Universität Göttingen

Mitglieder der Prüfungskommission:

Referent: Prof. Dr. Arnulf Quadt

II. Physikalisches Institut, Georg-August-Universität Göttingen

Koreferent: Prof. Dr. Claus Gößling

Lehrstuhl für Experimentelle Physik IV, Technische Universität Dortmund

Weitere Mitglieder der Prüfungskommission:

PD Dr. Ralf Bernhard

II. Physikalisches Institut, Georg-August-Universität Göttingen

Prof. Dr. Stan Lai

II. Physikalisches Institut, Georg-August-Universität Göttingen

apl. Prof. Dr. Michael Seibt

IV. Physikalisches Institut, Georg-August-Universität Göttingen

Jun.-Prof. Dr. Steffen Schumann

II. Physikalisches Institut, Georg-August-Universität Göttingen

Tag der mündlichen Prüfung: 02.08.2016 


\title{
Detector Development for the
}

\section{High Luminosity Large Hadron Collider}

\author{
Julia Rieger
}

\begin{abstract}
To maximise the discovery potential of the Large Hadron Collider, it will be upgraded to the High Luminosity Large Hadron Collider in 2024. New detector challenges arise from the higher instantaneous luminosity and the higher particle flux. The new ATLAS Inner Tracker will replace the current tracking detector to be able to cope with these challenges. Many pixel detector technologies exist for particle tracking, but their suitability for the ATLAS Inner Tracker needs to be studied. Active high-voltage CMOS sensors, which are produced in industrialised processes, offer a fast readout and radiation tolerance. In this thesis the HV2FEI4v2 sensor, which is capacitively coupled to the ATLAS Pixel FE-I4 readout chip, is characterised for the usage in the outer layers of the ATLAS Inner Tracker. Key quantities of this prototype module are studied, such as the hit efficiency and the subpixel encoding. The early HV2FEI4v2 prototype shows promising results as a starting point for further module developments. Active CMOS sensors serve as possible candidates for a cost effective pixel detector for the High Luminosity Large Hadron Collider.
\end{abstract}

II.Physik-UniGö-Diss-2016/01

II. Physikalisches Institut

Georg-August-Universität Göttingen 



\title{
Detector Development for the
}

\section{High Luminosity Large Hadron Collider}

\author{
Julia Rieger
}

\section{Zusammenfassung}

Um das Entdeckungspotential des Large Hadron Colliders auszunutzen, wird er beginnend 2024 zum High Luminosity Large Hadron Collider ausgebaut. Neue Detektorherausforderungen entstehen durch die höhere instantane Luminosität und den höheren Teilchenfluss. Der neue ATLAS Inner Tracker wird den aktuellen Spurdetektor ersetzen, um mit diesen Herausforderungen umzugehen. Es gibt viele Pixeldetektortechnologien zur Teilchenspurerkennung, jedoch muss ihre Eignung für den ATLAS Inner Tracker untersucht werden. Aktive Hochspannungs-CMOS-Sensoren, die in industriellen Prozessen produziert werden, bieten eine schnelle Auslese und Strahlenhärte. In dieser Arbeit wird der HV2FEI4v2-Sensor, der kapazitiv mit dem ATLAS-FE-I4-Auslesechip gekoppelt ist, dahingehend charakterisiert, ob er für eine Verwendung in einer der äußeren Lagen des ATLAS Inner Tracker geeignet ist. Schlüsselgrößen des Prototypens, wie die Treffereffizienz und die Subpixelentschlüsselung, werden untersucht. Der frühe HV2FEI4v2Prototyp zeigt vielversprechende Ergebnisse, die als Ausgangspunkt für weitere Entwicklungen dienen. Aktive CMOS-Sensoren stellen einen möglichen Kandidaten für einen kosteneffizienten Detektor für den High Luminosity Large Hadron Collider.

II.Physik-UniGö-Diss-2016/01

II. Physikalisches Institut

Georg-August-Universität Göttingen 



\section{Contents}

1 Introduction 1

2 The Large Hardron Collider and the ATLAS Experiment 3

2.1 Physics at the LHC 3

2.1.1 Standard Model of Particle Physics 3

2.1.2 Limitations of the Standard Model 4

2.2 The Large Hardron Collider 5

2.3 The ATLAS Experiment 7

2.3.1 Detector Requirements 7

2.3.2 Coordinate System 8

2.3.3 Trigger System 8

2.3.4 Muon Spectrometer 9

2.3.5 Calorimetry 9

2.3.6 Tracking 10

2.4 Upgrades of LHC and ATLAS 11

2.4.1 Physics Goals 12

2.4.2 Phase-0 Upgrade 12

2.4.3 Phase-I Upgrade 12

2.4.4 Phase-II Upgrade 13

\section{Silicon Pixel Detectors 15}

3.1 Interaction of Particles with Matter 15

3.1.1 Charged Particles in Matter 15

3.1.2 Photons in Matter 17

3.1.3 Multiple Scattering 17

3.2 Vertex Measurement 18

3.2.1 Spatial Resolution 18

3.2.2 Vertex Resolution 18

3.3 Signal Generation 19

3.3.1 Fundamental Properties of Silicon 19

3.3.2 pn-Junction 19

3.3.3 Charge Collection 20

3.3.4 Radiation Damage in the Sensor Material 21 
3.4 Readout Electronics 23

3.4.1 Noise in Analogue Readout Electronics 24

3.4.2 Radiation Damage in the Readout Electronics 24

4 Pixel Detector Technologies 27

4.1 Current ATLAS Pixel Detector 27

4.1.1 Sensor 28

4.1.2 Readout Chip 29

4.2 Insertable B-Layer 30

4.2.1 Sensor 31

4.2.2 Readout chip 32

4.3 Four-Chip Modules 33

4.4 Active High-Voltage CMOS Sensors 39

5 Readout System Integration and Measurement Set-Up 43

5.1 USBpix Readout System 43

5.1.1 STcontrol 44

5.1.2 Standard Scans 45

5.2 CMOS Sensor Integration into STcontrol 47

5.2.1 PixModule 47

5.2.2 PixController 48

5.2.3 USBpixdll 48

5.2.4 Newly Implemented Scans 49

5.3 Test Beam Set-Up 49

5.3.1 Test Beam Facility 50

5.3.2 Telescope Sensors 50

5.3.3 Triggering and Data Synchronisation 51

5.3.4 Data Acquisition Software 52

5.3.5 Track Reconstruction 52

5.3.6 Analysis Framework 53

6 HV2FEI4 Characterisation 55

6.1 Configuration 56

6.2 Parameter Studies 60

6.2.1 HV2FEI4 Amplifier Characterisation 60

6.2.2 Validation of the STcontrol Implementation 62

6.2.3 HV2FEI4 Comparator Threshold Studies 64

6.2.4 ToT Studies towards Subpixel Decoding 70

6.2.5 IV Measurement 73

6.3 Hit Efficiency Studies with One Subpixel Type 74

6.3.1 Comparison of the Three Subpixel Types 74

6.3.2 Studies with Subpixel Type 184 
6.4 Subpixel Decoding 88

6.4.1 Subpixel Position 88

6.4.2 Subpixel Mapping 90

6.4.3 Comparison of Cut and Likelihood Method for Subpixel Mapping 94

6.5 Hit Efficiency Studies with Complete Sensor 104

7 Conclusion and Outlook 111

7.1 Conclusion 111

7.1.1 Four-Chip Module 111

7.1.2 CCPD Module with HV2FEI4v2 Sensor 112

7.2 Outlook 113

A Additional Plots 115

Bibliography 123

Acknowledgements 129 



\section{Introduction}

When you open your eyes in the morning, you see all objects around you. Your eyes detect the light that is scattered off the objects. Generally, electromagnetic radiation interacts with matter. Yet there is more around you. A huge amount of elementary particles pass unnoticed through your body every day. About two muons per second pass through your hand the incredible number of about 100 billion neutrinos per second cross an area as small as your thumbnail. These particles are not noticed by our human senses. Though, via interaction with matter these particles can be detected. Thus, particle detectors yield sensory access to an invisible part of reality.

Depending on their environment, particle detectors serve many purposes. The measurement of the trajectory of charged particles is one application. One of the first Nobel Prizes in Physics for a particle detector was given to C.T.R. Wilson in 1927 "for his method of making the paths of electrically charged particles visible by condensation of vapour" [1] The cloud chamber subsequently became an important tool in physics.

In the experiments for particle physics, particle properties such as energy, trajectory and momentum are measured. Experiments like ATLAS (see Chapter 2) combine many different detector technologies to identify all passing particles. For high-resolution tracking of particles in the innermost layers, silicon pixel detectors are used. The interactions of the particles with matter generate a signal in the detectors, which is then read out (see Chapter 3).

High energy physics investigates the nature of the elementary particles and their interactions. Our current knowledge of particle physics is reflected in the Standard Model (see Chapter 2). It is a powerful theory describing the phenomena in particle physics, but some limitations indicate that it needs to be extended. In particle collisions at the Large Hadron Collider at the European Organisation for Nuclear Research (CERN), conditions are recreated matching one millionth of a millionth of a second after the beginning of the universe. At the Large Hadron Collider particles collide and the Standard Model of Particle Physics is probed with the results from the detectors. The achievements by the Large Hadron Collider experiments, especially the discovery of the Higgs boson motivate further measurements. To maximise the discovery potential of the Large Hadron Collider, it will be upgraded to the High Luminosity Large Hadron Collider.

Many different pixel detector technologies exist for particle tracking (see Chapter 4). The decision for a certain technology is based on challenges defined by the detector environment, such as the high particle flux at the Large Hadron Collider. Furthermore, specific detector functionalities are required including a high detector granularity and fast readout 
electronics. Thus, the signatures can be identified that are characteristic of the physics processes.

To determine the feasibility and to characterise the performance of different prototype modules, laboratory and test beam measurements (see Chapter 5) are performed in the context of this thesis. This thesis' focus is on active high-voltage CMOS sensors (see Chapter 6), which are industrially available and feature high breakdown voltages. These high voltages allow the creation of a depletion zone with a thickness in the order of $10-100 \mu \mathrm{m}$, which is suitable for particle detection. Sensor productions on larger wafers are possible and potentially reduce the costs compared to passive hybrid detectors. 


\section{The Large Hardron Collider and the ATLAS Experiment}

\subsection{Physics at the LHC}

\subsubsection{Standard Model of Particle Physics}

The Standard Model of Particle Physics (SM) [2-5] reflects our current knowledge of all elementary particles and their interactions found in the last decades. Since the formulation of the SM in the 1970s, its predictions and all performed measurements agree with a high level of accuracy. Starting from separate theories for electromagnetic, weak and strong force, the SM developed into a unified quantum field theory based on a local $S U(3)_{C} \times S U(2)_{L} \times U(1)_{Y}$ gauge symmetry.

Divided into fermions (half-integer spin) and bosons (integer spin), the SM contains 17 particles (and their respective antiparticles). Figure 2.1 shows all SM particles and their masses, charges and spins. Gauge particles are spin-1 bosons, which mediate three

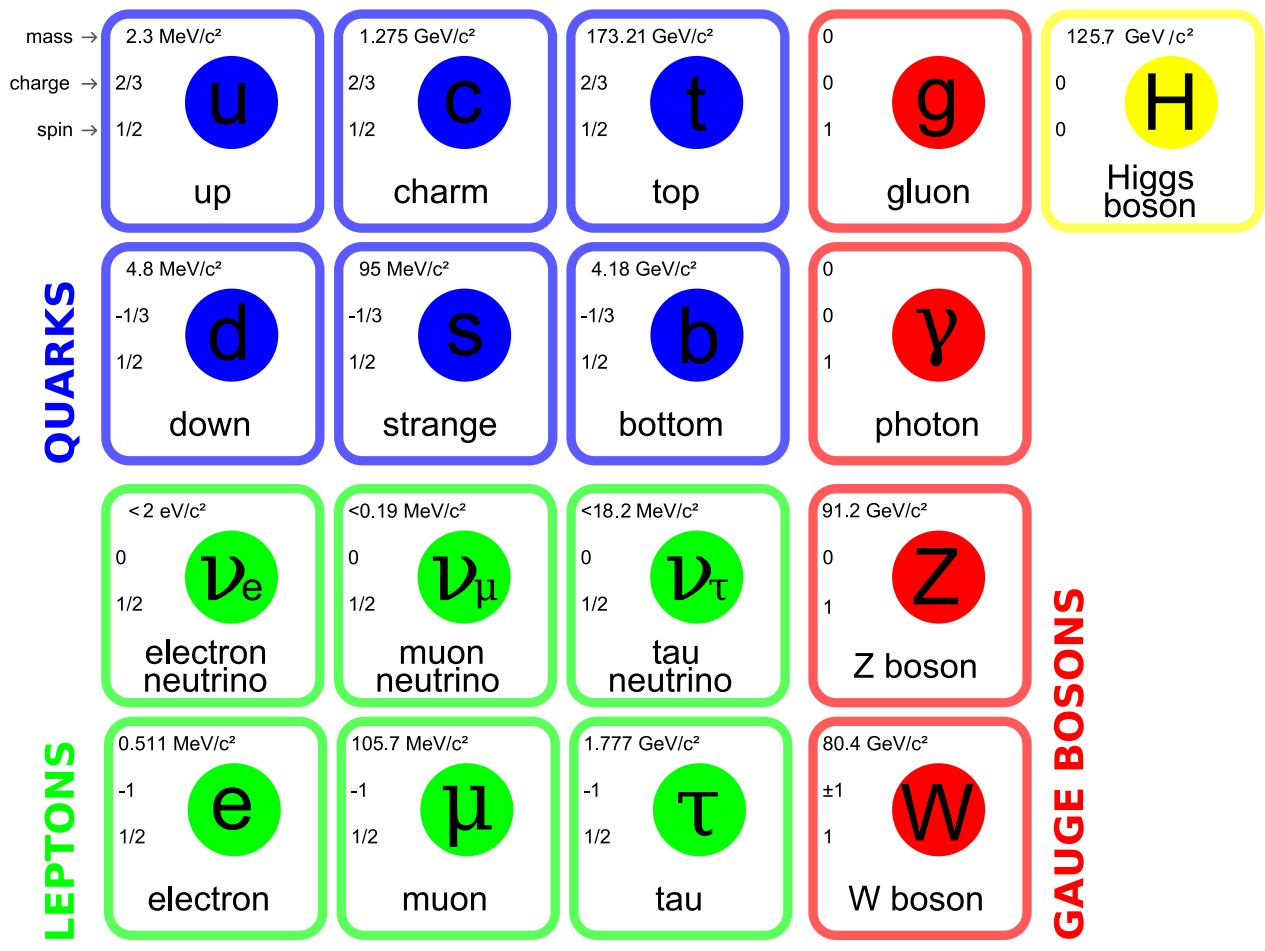

Figure 2.1: Elementary particles in the SM. The mass values are taken from [6]. 
out of four fundamental interactions. Gravity is not included in the SM, but can be neglected at the mass scale of the elementary particles. The mediator of the electromagnetic force is the massless photon $(\gamma)$, coupling to electrical charge. The massive $W^{ \pm}$and $Z^{0}$ bosons are the mediators for the electroweak interaction. In addition, the eight massless gluons $(g)$, which are responsible for the interactions via the strong force, represent the possible combinations of colour and anticolour.

The last experimentally discovered SM particle is the Higgs boson, which has spin 0 . In 2012, the Higgs boson was discovered by the ATLAS and CMS experiments $[7,8]$, confirming the Higgs mechanism [9-11]. This process is responsible for electroweak symmetry breaking and mass generation.

The fermions are subdivided into leptons and quarks, which are arranged in generations with increasing masses. The higher mass of the second and third generation fermions explains, why they are unstable and have a finite lifetime, whereas the first generation particles are stable and form everyday-matter. During the short lifetime of the particles, they can travel a significant distance at the speed of light. For example, B-hadrons, having a lifetime of the order of $10^{-12} \mathrm{~s}$, travel up to several $\mathrm{mm}$ before decaying. These decay vertices can be used to identify such particles.

Depending on their properties, the particles interact via different forces. All fermions interact through the weak interaction. Particles that carry an electrical charge can participate in electromagnetic interactions, as there are charged leptons with an electrical charge of -1 and quarks having an electrical charge of $\frac{2}{3}$ or $-\frac{1}{3}$. In addition, quarks come in three different colours (and anti-colours). The colour is an additional quantum number and is required for interactions via the strong force. Hadronic matter is colourless, hence quarks group to baryons (three-quark state) or mesons (quark-antiquark state).

\subsubsection{Limitations of the Standard Model}

The SM is a powerful theory describing the physical phenomena in particle physics. But some limitations indicate that it needs to be extended or embedded into a larger theory. This theory possibly includes gravity or unifies the strong and the electroweak interaction. The observation of neutrino oscillation shows that neutrinos have a non-zero mass $[12,13]$, which contradicts the SM assumption of massless neutrinos. Furthermore, astrophysical observations infer that dark matter exists. For example, the behaviour of the rotation velocity of rotating galaxies for large radii can only be explained, if dark matter is considered in addition to the visible mass of the galaxies [14]. Moreover, direct empirical proof of the existence of dark matter exists from gravitational lensing measurements [15]. The measurements of the cosmic microwave background temperature fluctuations show that only about $5 \%$ of the universe is SM matter and about $25 \%$ is dark matter [16].

Many different theories extend the SM, such as Supersymmetry (SUSY), which introduces a symmetry between bosons and fermions. The SM is an incomplete theory, thus new physics must exist at the TeV-scale. To answer the open questions, the Large Hadron Collider was built, having large discovery potential. Collider-based direct dark matter searches and searches for SUSY particles are performed among many other searches and measurements. 


\subsection{The Large Hardron Collider}

The Large Hardron Collider (LHC) [17] is located at the European Organisation for Nuclear Research (CERN) close to Geneva. It is a proton-proton collider with a design centre-of-mass energy of $14 \mathrm{TeV}$. Furthermore, the LHC can collide heavy ions. Situated in the former Large Electron-Positron Collider (LEP) tunnel, the LHC is about $100 \mathrm{~m}$ below ground and has a circumference of about $27 \mathrm{~km}$.

Radio frequency superconducting cavities accelerate protons with an accelerating gradient of $16 \mathrm{MV} / \mathrm{m}$. Because of this technique no continuous beam is possible, but the protons are grouped into bunches. The design values for the number of protons in one bunch and the number of colliding bunches are $N_{p}=1.15 \cdot 10^{11}$ and $n_{b}=2808$, respectively. The bunches are separated by $25 \mathrm{~ns}$, which corresponds to a collision rate of $f=40 \mathrm{MHz}$.

Superconducting bending and focusing magnets, providing a magnetic field of about $8.4 \mathrm{~T}$, guide the beam particles around the ring. The particles circulate in two separate beam pipes in opposite directions before they collide. These magnets are cooled with superfluid helium to a temperature of $1.9 \mathrm{~K}$ to keep the superconducting state.

The key parameters of the circular machine are the particle energy and the luminosity. The latter is a machine parameter given by

$$
L=\frac{N_{p} \cdot f \cdot n_{b}}{4 \pi \cdot \sigma_{x} \cdot \sigma_{y}}
$$

with $N_{p}$ the number of protons per bunch, $f$ the revolution frequency, $n_{b}$ the number of bunches per beam and $\sigma_{x} \cdot \sigma_{y}$ the beam dimensions at the interaction point.

To observe rare events, the luminosity must be high enough. For instance, the crosssections of the Higgs boson productions are more than three orders of magnitude smaller than the cross-sections of $W$ or $Z$ boson production. In general, the event rate is

$$
\frac{\mathrm{d} N}{\mathrm{~d} t}=L \times \sigma
$$

with $\mathrm{d} N$ the number of events per given time interval $\mathrm{d} t$ depending on the luminosity $L$ and the cross-section $\sigma$. If a total cross-section of 100 mbarn $\left(1 \mathrm{barn}=10^{-24} \mathrm{~cm}^{2}\right)$ is assumed for proton-proton collisions, the event rate for a design luminosity of $10^{34} \mathrm{~cm}^{-2}$ $\mathrm{s}^{-1}$ is in the order of $10^{9}$ events per second. Considering these parameters, the number of colliding proton pairs per bunch crossing is about 25 .

In addition, because of the nature of proton-proton collisions, cross-sections of rare processes are many orders of magnitude smaller compared to jet production cross-sections. This nature is a challenge for the LHC experiments.

The total number of particles created at an accelerator is proportional to the integrated 


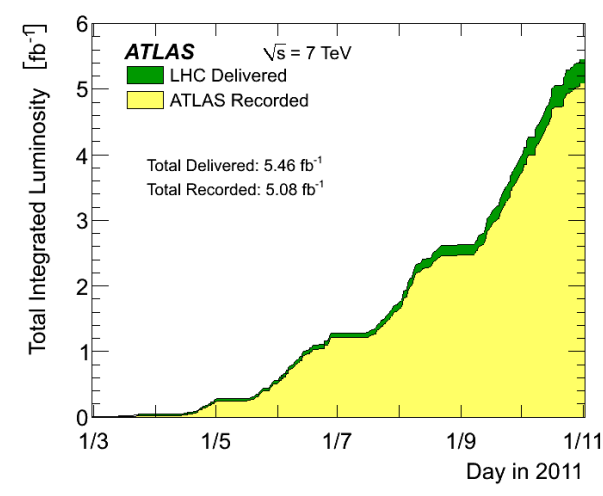

(a) 2011 [18].

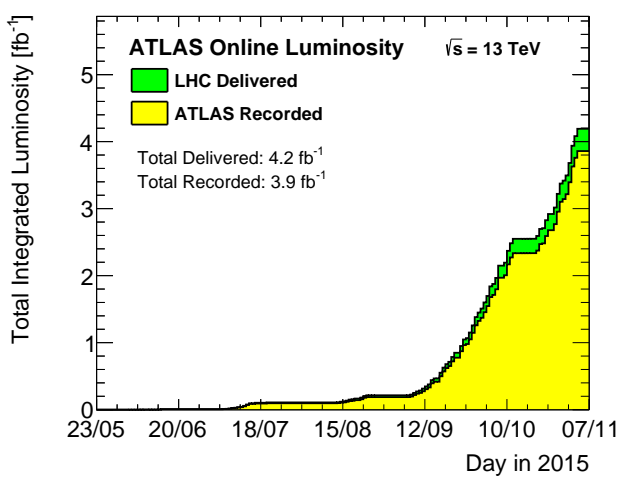

(c) 2015 [19].

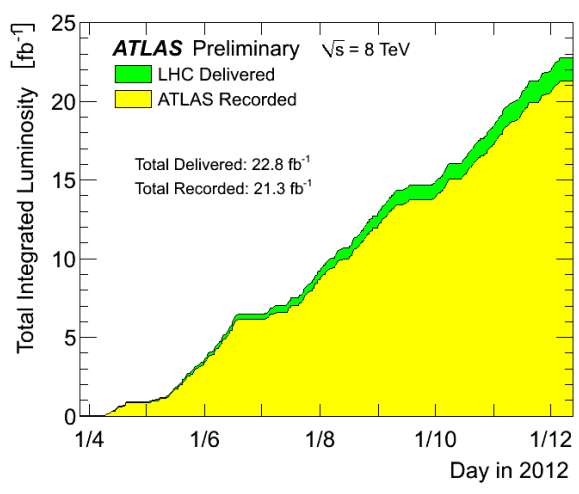

(b) 2012 [18].

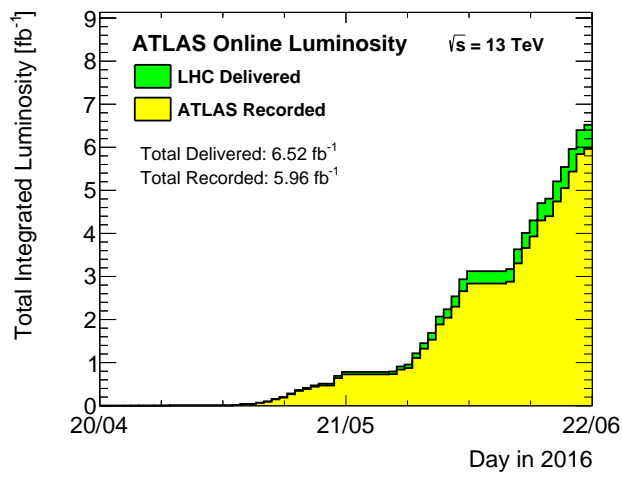

(d) 2016 [19].

Figure 2.2: Total integrated luminosity versus time delivered to (green) and recorded by ATLAS (yellow) during stable beams and for proton-proton collisions at $7 \mathrm{TeV}$ centre-of-mass energy in 2011 (a), at $8 \mathrm{TeV}$ center-of-mass energy in 2012 (b) and at 13 TeV centre-of-mass energy in 2015 (c) and 2016 (d).

luminosity

$$
\mathscr{L}=\int L(t) \mathrm{d} t
$$

with its unit $\mathrm{cm}^{-2}$ expressed in inverse femtobarn $\left(\mathrm{fb}^{-1}\right)$.

The first collisions took place in 2009 with a centre-of-mass energy of $900 \mathrm{GeV}$, which was increased to $7 \mathrm{TeV}$ for 2010 and 2011. In 2012 the energy was increased to $8 \mathrm{TeV}$ and since 2015 the protons collide at a centre-of-mass energy of $13 \mathrm{TeV}$. The total integrated luminosity that was delivered by the LHC and recorded by the ATLAS Experiment in 2011, 2012, 2015 and 2016 can be seen in Figure 2.2.

With this luminosity rare processes like the Higgs boson production were observed. To detect these events, fast responding, finely segmented and radiation hard detectors are needed. The locations of the main detectors are at the four interaction points of the LHC. The two multi-purpose detectors ATLAS (A Toroidal LHC ApparatuS, see Section 2.3) and 


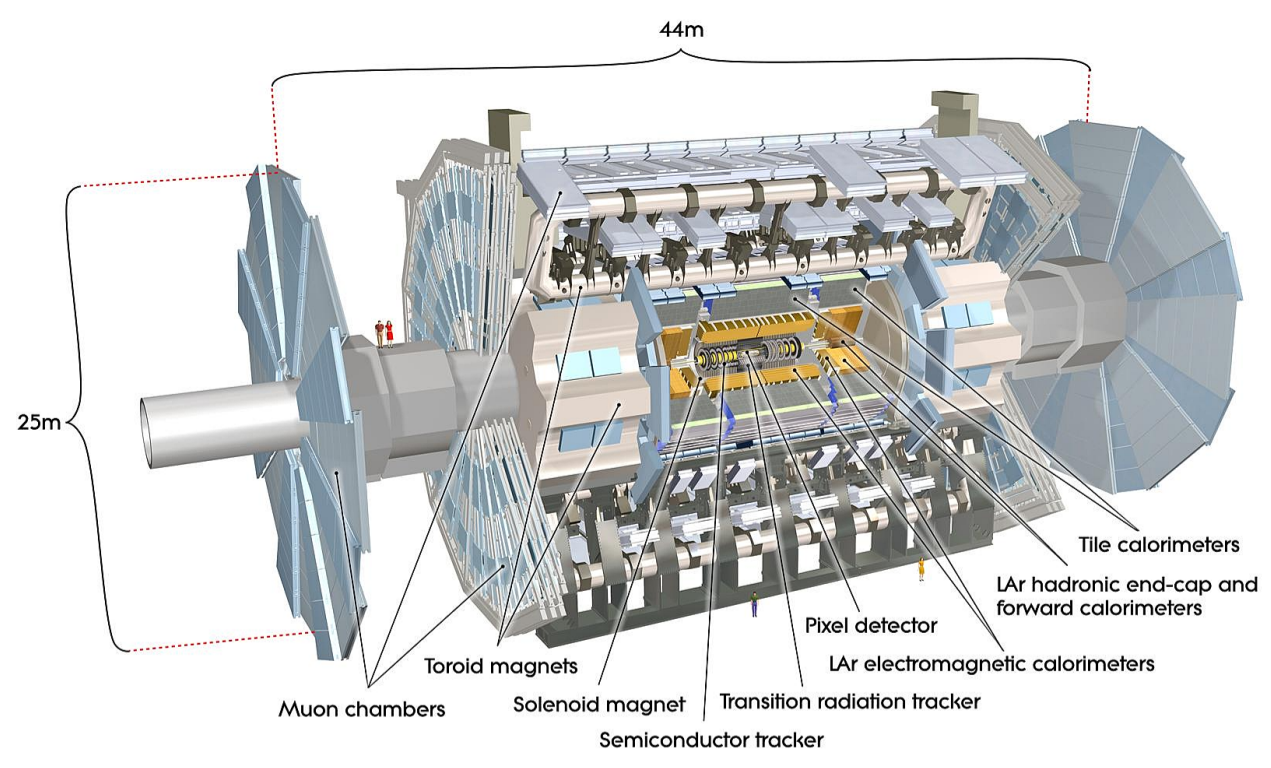

Figure 2.3: Schematic view of the ATLAS Detector. The dimensions and the different subdetectors are indicated. Further details can be found in the text.

CMS (Compact Muon Solenoid) perform high precision measurements of the SM physics, the Higgs boson and new physics. LHCb (Large Hadron Collider beauty) concentrates on matter-antimatter balance and ALICE (A Large Ion Collider Experiment) on heavy ion collisions.

\subsection{The ATLAS Experiment}

The ATLAS Detector [20] is the largest detector at the LHC, with a width of $44 \mathrm{~m}$, a diameter of $25 \mathrm{~m}$ and a weight of about $7000 \mathrm{t}$. It consists of several layers of tracking, calorimetry and muon detectors (see Figure 2.3). Together with the magnet and trigger system, it is capable of dealing with the large challenges of the LHC, mentioned in Section 2.2 and fully exploit the physics capability.

\subsubsection{Detector Requirements}

The main detector requirements are the identification of experimental signatures characteristic of the physics processes, such as secondary vertices or missing transverse momentum. Furthermore, excellent particle-identification capabilities are required. To cope with the high particle flux, fast, radiation-hard electronics and sensor elements are essential. Because of the nature of proton-proton collisions several proton pairs collide per interaction. To reduce the influence of these overlapping events, a high detector granularity is necessary. A large acceptance in pseudorapidity with almost full azimuthal angle coverage 
is required to detect all particles and to identify neutrinos and new particles escaping the detector.

To separate particles coming from different interaction processes, it is essential for the tracking system to have a good reconstruction efficiency and charged-particle momentum resolution. For offline identification of $\tau$-leptons and $b$-jets, tracking detectors for vertex reconstruction close to the interaction region are required to observe secondary vertices. The calorimeter system precisely measures the particle energy and enables the reconstruction of the total transverse momentum. For electron and photon identification and measurements, very good electromagnetic calorimetry is required as well as full-coverage hadronic calorimetry for the jet measurements. Good muon identification and momentum resolution over a wide range of momenta is necessary. With the magnetic field the charge of muons is determined. In addition, a highly efficient triggering system is essential to achieve an acceptable trigger rate for most physics processes of interest.

\subsubsection{Coordinate System}

For an explicit geometrical description of the detector a right-handed coordinate system is used with the origin at the nominal interaction point. The $z$-axis is along the beam direction. The $x-y$-plane is transverse to the beam direction with the positive $x$-axis pointing from the interaction point to the centre of the LHC ring. The positive $y$-axis points upwards. The azimuthal angle $\phi$ is measured around the beam axis and the polar angle $\theta$ is the angle from the beam axis. The rapidity is defined as

$$
y=\frac{1}{2} \ln \left(\frac{E-p_{z}}{E+p_{z}}\right)
$$

with the energy $E$ and the $z$ momentum component $p_{z}$. The advantage of this description is that rapidity differences are invariant with respect to Lorentz boosts along the z-axis. For relativistic particles with $p>>m$ it is more handy to use the pseudorapidity

$$
\eta=-\ln \left(\tan \frac{\theta}{2}\right)
$$

For massless objects these two quantities are equivalent.

\subsubsection{Trigger System}

Considering the collision rate of the proton bunches, about a billion proton-proton events are produced per second. Most events just contain well-known proton scattering events and the size per event is approximately 1.3 MB. The resulting total data to be recorded exceeds the amount of data that can be stored permanently, which is in the order of 200 events per second. Therefore, the ATLAS Trigger System [20] decides, which events to keep. It reduces the data rate from $40 \mathrm{MHz}$ to about $200 \mathrm{~Hz}$ in three steps.

The first step is the hardware-based Level 1 Trigger (L1), which decreases the rate from $40 \mathrm{MHz}$ to $75 \mathrm{kHz}$ using calorimeter and muon system information. The data are stored in pipeline buffers inside the detector until the L1 decision is made, which takes about $2.5 \mu$ s. From the accepted objects regions of interest (RoI) are built, which are small 
regions in the $\eta-\phi$ space centred around the object.

The Level 2 Trigger (L2) is a software trigger that restricts itself to the RoI and uses the full resolution and granularity of all detectors in this region. After the average event processing time of about $40 \mathrm{~ms}$ and a reduction of the rate from $75 \mathrm{kHz}$ to about $2 \mathrm{kHz}$, an event is built.

The last step of the trigger system is the Event Filter (EF), which reduces the rate to about $200 \mathrm{~Hz}$. The full event information is accessed and offline analysis algorithms are used. An average event processing time is of the order of $4 \mathrm{~s}$. These data sets are then sent to mass storage.

\subsubsection{Muon Spectrometer}

The two functionalities provided by the ATLAS Muon Spectrometer [20] are fast triggering of muons, originating from the interaction point, and the measurement of the muon momentum with high precision using the track curvature. The muon spectrometer is the outermost part of the ATLAS Detector and only muons can reach this part. All other known particles, besides neutrinos, are stopped in the calorimeters. The layout of the muon system is based on the magnetic deflection of the muon trajectories in the large superconducting air-core toroid magnets, whose field reaches up to $|\eta|=2.7$. The magnetic field has an average value of $0.5 \mathrm{~T}$ in the barrel region and can reach up to $3.5 \mathrm{~T}$ in the endcap region.

To manage the diverse tasks of triggering and tracking in different detector environments, four detector types exist. Detectors for triggering need to be very fast and to have a good time resolution. Precise tracking detectors with a good spatial resolution are needed to measure the bending of the muon track in the magnetic field. In addition, the special requirements at positions close to the beam pipe have to be taken in account. Because of the higher particle rate at high $\eta$ regions different detectors are needed there.

For triggering in the central region $(|\eta|<1.05)$ Resistive Plate Chambers are used, which are finely segmented gaseous parallel electrode-plates, operating in avalanche mode and having an intrinsic time resolution of $1.5 \mathrm{~ns}$. The Thin Gap Chambers in the forward regions $(1.05<|\eta|<2.7)$ are multi-wire proportional chambers. They can cope with higher counting rates, but have an intrinsic time resolution of $4 \mathrm{~ns}$. In addition to triggering, the Resistive Plate Chambers and Thin Gap Chambers are also used to provide tracking information.

Drift tubes filled with an $\mathrm{Ar} / \mathrm{CO}_{2}$-gas mixture, which are called Monitored Drift Tubes, are used in the barrel region $(|\eta|<2)$ for high resolution tracking with a stand-alone spatial resolution of $35 \mu \mathrm{m}$. Cathode Strip Chambers, which are multi-wire proportional chambers with strip cathodes, are utilised in the region $2<|\eta|<2.7$ having a spatial resolution of $40 \mu \mathrm{m}$.

\subsubsection{Calorimetry}

The calorimeter system measures the deposited energy of particles by absorbing them. It is divided into an electromagnetic and a hadronic part. The electromagnetic calorimeter 
is specifically designed to measure the energy of particles that interact primarily via the electromagnetic interaction. To measure particles that interact via the strong nuclear force, a hadronic calorimeter is used.

In the ATLAS Detector, both parts are sampling calorimeters with active and passive material, so the detector does not become too large. The passive material needs to have a high density, so that the high energetic particles initiate a cascade of secondary particles. During this particle shower low-energy particles are produced, which are then stopped and absorbed. The active material detects the shower. To measure the total energy of the initial particles, it must provide good containment for electromagnetic and hadronic showers and limit the punch-through into the muon system. In addition, a good energy resolution and a good linearity must be achieved in the measurement performance over a large energy range.

The electromagnetic calorimeter, covering the pseudorapidity region $|\eta|<3.2$, measures the energy of electrons and photons. Photons do not cause hits in the tracking detectors, so that their direction can only be determined by the point of impact in the calorimeter. Thus, the innermost calorimeter layer has a very fine granularity to perform precision measurements. Liquid argon, used as active material, is intrinsically radiation hard and is read out quickly. Lead is the absorber material of the electromagnetic calorimeter.

All hadrons are detected by hadronic calorimeters using different techniques suited for the widely varying requirements. The Tile Calorimeter covering $|\eta|<1.7$ uses steel as absorber and plastic scintillator tiles as active material. The higher pseudorapidity region $1.5<|\eta|<3.2$ is covered by the Hadronic End-cap Calorimeter. It shares the liquid argon cryostat with the electromagnetic calorimeter, but it uses copper as absorber. Because of its intrinsic radiation tolerance, the liquid argon technology was chosen again for the high pseudorapidity regions. Finally, the Forward Calorimeter covers the high pseudorapidity regions of $3.1<|\eta|<4.9$, which uses liquid argon and a dense tungsten matrix.

The total thickness of the electromagnetic calorimeter is more than 22 radiation lengths $\left(X_{0}\right)$ in the barrel and more than $24 X_{0}$ in the endcaps. The approximately ten interaction lengths $(\lambda)$ of the hadronic calorimeter provide good resolution for high energy jets.

\subsubsection{Tracking}

Stretching over a length of $6.2 \mathrm{~m}$ with a diameter of $2.1 \mathrm{~m}$, the Inner Detector (ID) is the innermost component of the ATLAS Detector. It is surrounded by a $2 \mathrm{~T}$ magnetic field generated by the central solenoid. At every bunch crossing about 1000 particles emerge from the collision point within $|\eta|<2.5$ entering the ID. Considering this large track density, fine granularity detectors are needed to achieve the required momentum and vertex resolution. Main challenges for the ID are high particle rates and the resulting radiation, which lead to further requirements for readout electronics and sensor material. The ID is divided into three subdetectors, which all need to be reliable, affordable and have as little material as possible in order not to influence the energy measurement in the calorimeter system.

The outermost part is the Transition Radiation Tracker (TRT), which is composed of about 300,000 straw-tubes embedded in a passive material and filled with a xenon-based gas 
mixture. On average, a particle crosses 36 straws for $|\eta|<2.1$ while passing the TRT, which has a spatial resolution of ca $130 \mu \mathrm{m}$. The TRT is not only for tracking, but it can also be used for electron identification. Electrons passing the passive material emit transition radiation due to different dielectric constants. This radiation can be detected by the straws.

With four barrel layers and nine endcaps on either side filling an area of about $60 \mathrm{~m}^{2}$, the Semiconductor Tracker (SCT) is the second tracking detector. Every module consists of two layers of single sided p-in-n microstrip sensors, which are rotated against each other by a stereo angle of $40 \mathrm{mrad}$ to get two-dimensional hit information. The resulting spatial resolution is $16 \mu \mathrm{m}$ in $R \phi$-direction and about $580 \mu \mathrm{m}$ along the $z$-direction.

Finally, the innermost component of the ID is the ATLAS Pixel Detector, which is needed for track and vertex reconstruction and b-tagging. It is divided into three barrel-shaped layers and three discs on either side. Because of its position closest to the beam pipe it faces the highest particle flux. The spatial resolution in $R \phi$-direction is $12 \mu \mathrm{m}$ and along the $z$-direction about $90 \mu \mathrm{m}$. Its total active area is about $1.7 \mathrm{~m}^{2}$ and it has about 80 million channels. A detailed description of the ATLAS Pixel Detector can be found in Section 4.1.

\subsection{Upgrades of LHC and ATLAS}

The achievements by the LHC experiments and especially the discovery of the Higgs boson motivate further measurements. To maximise the discovery potential of the LHC, it will be upgraded towards the High Luminosity Large Hardon Collider (HL-LHC). The plan is ultimately to achieve the luminosity of $7.5 \times 10^{34} \mathrm{~cm}^{-2} \mathrm{~s}^{-1}$ leading to up to 200 inelastic proton-proton collisions per bunch crossing [21]. Consequently, a total integrated luminosity of $3000 \mathrm{fb}^{-1}$ is supposed to be accumulated by 2035 . Figure 2.4 shows the upgrade schedule. The accelerator and the detector upgrades will be progressively installed during the next two major shut-downs, defined as LS2 (2019-2020) and LS3 (2024-2026) according to current planning.

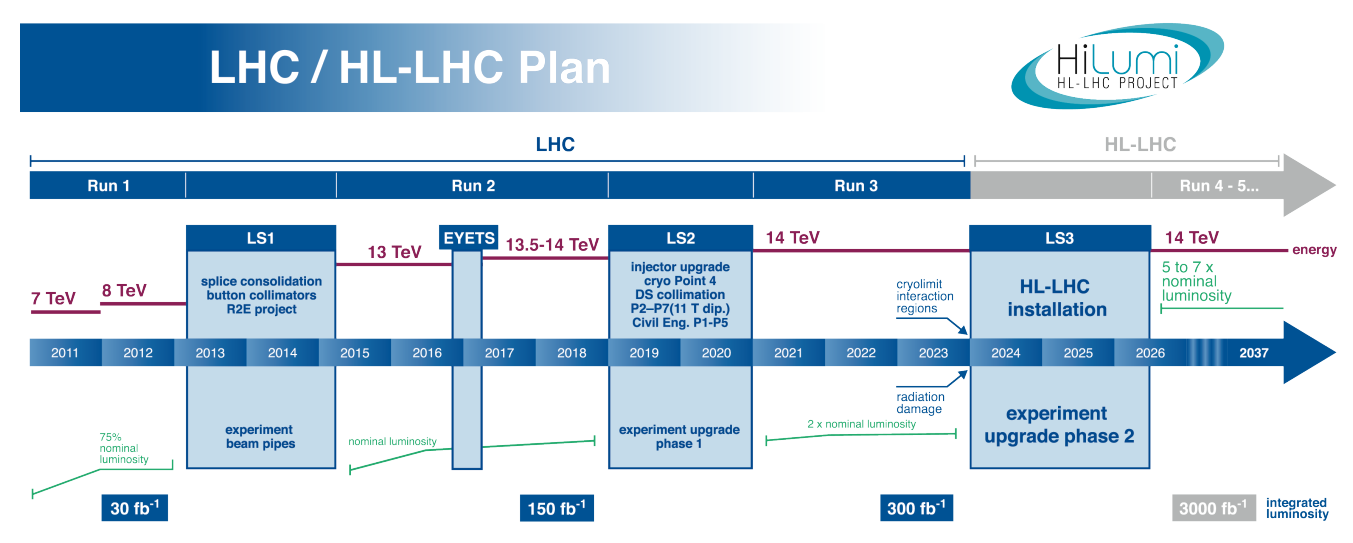

Figure 2.4: High Luminosity LHC plan including the foreseen centre-of-mass energy and luminosity [22]. 


\subsubsection{Physics Goals}

The benchmark physics processes that define the requirements on the detector components are electroweak and Higgs boson physics, searches for Supersymmetry and new physics processes. Precision measurements of the Higgs boson are performed through studies of all accessible Higgs boson production processes and decay final states. The SM predicts the coupling constants for the particles and a significant deviation would hint to new physics.

For example, the $t \bar{t} H$-process is the only way to directly study the top quark Yukawa coupling. To observe this process, a good b-tagging performance is necessary. Studies of the Higgs bosons produced via vector-boson fusion (VBF) with Higgs boson decays into gauge bosons $\left(H \rightarrow Z Z^{*} \rightarrow \ell \ell \ell \ell, H \rightarrow W W^{*} \rightarrow \ell v \ell v\right)$ require the reconstruction of the forward jets to distinguish it from the gluon fusion mode. Thus, the performance of the calorimeter and tracker systems in this very-forward region is essential. Furthermore, an excellent vertex measurement enables the rejection of background processes from different interactions. In addition, the measurement of the Higgs self-coupling and the subsequent reconstruction of the Higgs potential are important. The detection of the Higgs boson pair production via gluon-gluon fusion gives an insight into this process. The decay $H H \rightarrow b \bar{b} \gamma \gamma$ is one of the cleanest modes, but it has a small branching ratio. A good photon identification together with an again excellent b-tagging is required. For searches for new physics, the discovery relies on the performance of lepton triggers, missing transverse momentum reconstruction and b-jet identification. A more extensive discussion and performance studies can be found in [21] and [23].

The physics goals and consequently, the high luminosity with up to 200 interactions per beam crossing define the detector challenges. Furthermore, the forward region of the detector plays an important role. To acquire the statistics needed for rare physics processes, the upgrade of the triggering system and the particle identification and reconstruction is essential.

\subsubsection{Phase-0 Upgrade}

During the LS1 the magnet interconnections of the LHC machine were consolidated to run with the full design energy and prevent incidents.

The diameter of the beam pipe within the ATLAS Experiment was reduced and, using this space, a new innermost pixel layer, the Insertable-B-Layer (IBL, see Section 4.2) was introduced to improve the vertex measurement and b-jet identification. Furthermore, the complete Pixel Detector was revised and the full functionality was recovered.

\subsubsection{Phase-I Upgrade}

In the LS2 the LHC will be prepared to increase its luminosity to $2.2 \times 10^{34} \mathrm{~cm}^{-2} \mathrm{~s}^{-1}$, which is two times the design luminosity and up to 80 interactions per bunch-crossing are expected. To achieve this luminosity, the injector system needs to be replaced and the collimation system will be improved.

The ATLAS Experiment will improve the trigger system to keep a low trigger threshold for isolated leptons, required for a good physics performance. To handle the up to 80 
pile-up events, the background from jets misidentified as electrons must be reduced. Thus, the trigger electronics of the calorimeter trigger system will be changed, resulting in an improvement of the granularity of the calorimeter information for electron and jet reconstruction by a factor of 10 . The New Small Wheel will replace the first endcap of the muon spectrometer to enable muon tracking in the forward region and to improve the triggering. In addition, the Fast Track Trigger will implement a track-based trigger decision. With these improvements the collected data sample is expected to be approximately $300 \mathrm{fb}^{-1}$.

\subsubsection{Phase-II Upgrade}

The Phase-II Upgrade during the LS3 is the final step towards the HL-LHC. As already introduced, the luminosity is supposed to be $7.5 \times 10^{34} \mathrm{~cm}^{-2} \mathrm{~s}^{-1}$. The machine technologies to realise this high luminosity are under investigation and development and will require changes in all components from the superconducting cavities to the focusing magnets. The HL-LHC environment together with the physics program with the large variety of opportunities for discoveries and precision measurements define the detector requirements. It is essential that all detector components maintain and improve their performance in triggering and precisely reconstructing the full range of physics objects over as large a pseudorapidity region as possible.

To preserve the acceptance of key signatures such as $W$ and $Z$ bosons and $t \bar{t}$-pairs, a low trigger threshold needs to be kept. Consequently, a first trigger rate of at least $500 \mathrm{kHz}$ is required. A Level 0 trigger will be introduced, fulfilling these criteria, followed by a Level 1 trigger, using information from the tracking system and reducing the rate to about $200 \mathrm{kHz}$. The readout electronics of all detector components will be replaced. In the following, the consequences for the tracking detector will be introduced. For details and the upgrades of other detector components see [23].

The presence of up to 200 pile-up events will produce a multiplicity of more than 1000 tracks per unit of rapidity in the tracking detector. Facing this immense occupancy, the end of lifetime criteria for the components of the Inner Detector will be reached and a complete replacement of the tracking system is needed. The requirements on the detector depend on the distance to the interaction point. Figure 2.5 shows a baseline layout of the new all silicon Inner Tracker (ITk). In the Letter of Interest [23], it is suggested to have four pixel layers at small radii for pattern recognition and vertex measurements followed by three short-strip layers and two long-strip layers. The forward regions will be covered by six pixel disks and seven strip disks extending up to $|\eta|=2.7$. Different layout scenarios are under investigation reaching a rapidity coverage up to $|\eta|=4$.0. The layout is optimised to achieve the required momentum resolution by maximising the length of the trajectory inside the magnet. To cope with the occupancy, a significantly finer granularity is needed with smaller pixel sizes of $25 \times 150 \mu \mathrm{m}^{2}$ and thinner detectors. Consequently, the number of tracks per pixel is reduced. For a better precision tracking, tracking inefficiency and multiple scattering need to be limited by minimising the overall tracker material.

Due to huge particle fluences in the regions closest to the interaction point, the detector 


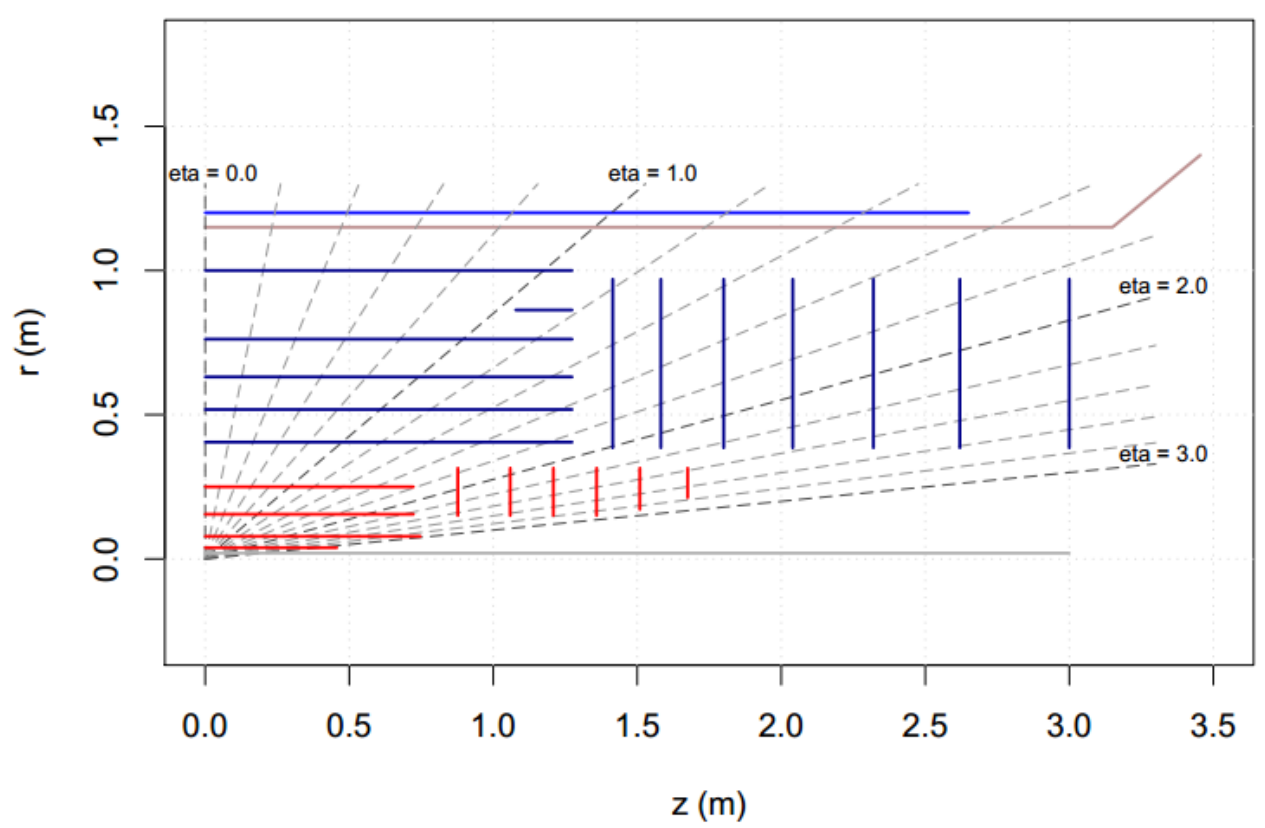

Figure 2.5: The baseline layout of the new ATLAS Inner Tracker showing the coverage of the pixel detector in red and strip detector in blue. The outermost blue line represents the magnet [23].

requirements on radiation hardness are extreme. Two important quantities of interest for understanding radiation damage in the silicon detectors and electronics are the $1 \mathrm{MeV}$ neutron equivalent fluence and the ionising dose. The predictions for the maximum fluence and ionising dose for $3000 \mathrm{fb}^{-1}$ in the centre of the innermost barrel layer is $1.4 \times 10^{16} n_{\mathrm{eq}} / \mathrm{cm}^{2}$ and 7.7 MGy [23]. In the outer layers of the pixel detector the fluence is approximately one order of magnitude smaller, $1.7 \times 10^{15} n_{\mathrm{eq}} / \mathrm{cm}^{2}$ and the ionising dose decreases to $0.9 \mathrm{MGy}$. Thus, the usage of different pixel technologies is motivated for the inner and outer layer. Especially, in view of the large pixel detector area of about $8.2 \mathrm{~m}^{2}$ with more than 600 million readout channels, more cost effective detectors will be needed. 


\section{Silicon Pixel Detectors}

Silicon pixel detectors are chosen for high-resolution particle tracking detectors in high energy physics. They generate the necessary signal height from passing particles and ensure a fast readout. Furthermore, they are mechanically stable, but have a small material budget, which is required for good vertex resolution.

\subsection{Interaction of Particles with Matter}

Particles behave differently while passing through matter. Depending on the properties of the particles such as charge, mass and energy, various processes determine their signatures in matter. These mechanisms are shown in the following.

\subsubsection{Charged Particles in Matter}

If a charged particle with a mass well above the electron mass $\left(M \gg m_{e}\right)$ penetrates the material, the main process of energy loss is ionisation. The average energy loss per distance is described by the Bethe-Bloch formula [24,25]

$$
-\left\langle\frac{\mathrm{d} E}{\mathrm{~d} x}\right\rangle=2 \pi N_{A} r_{e}^{2} m_{e} c^{2} \frac{Z}{A} \frac{z^{2}}{\beta^{2}}\left\{\ln \left(\frac{2 m_{e} c^{2} \beta^{2} \gamma^{2} W_{\max }}{I^{2}}\right)-2 \beta^{2}-\delta-2 \frac{C}{Z}\right\},
$$

where $r_{e}$ is the classical electron radius, $m_{e}$ the electron mass, $N_{A}$ Avogadro's number, $I$ the mean excitation potential, $Z$ the atomic number of the absorbing material, $A$ atomic weight of absorbing material, $z$ the charge of an incident particle in units of $e, \delta$ the density correction, $C$ the shell correction and finally the maximum energy transfer $W_{\max }$ in a single collision. By usual convention, the average energy loss is normalised to the density $\rho$ of the material without explicit notification.

The dependency of $\left\langle\frac{\mathrm{d} E}{\mathrm{~d} x}\right\rangle$ on $\beta \gamma$ is illustrated in Figure 3.1. For $\beta \gamma<0.1$ other processes than ionisation dominate the energy loss. After the maximum the $1 / \beta^{2}$-term describes the behaviour until a minimum at $\beta \gamma=3.5$ is reached. Particles with a momentum causing this energy loss of $\left\langle\frac{\mathrm{d} E}{\mathrm{~d} x}\right\rangle \approx 1.5 \frac{\mathrm{MeV} \mathrm{cm}}{\mathrm{g}}$ are called minimum ionising particles (MIP). The slow rise following the minimum is proportional to $\ln \beta \gamma$.

The Bethe-Bloch formula is derived assuming that particles passing the material are heavier than the shell electrons. For electrons a modification is necessary, because of the equal mass of the interacting particles, as well as the fact that the incident electron and the shell electron are quantum mechanically indistinguishable.

A contribution from Bremsstrahlung needs to be considered. This electromagnetic radiation is emitted if an electron is scattered in the field of a nucleus. Because of the acceleration of the electron, radiation is emitted. Bremsstrahlung is the dominating mech- 


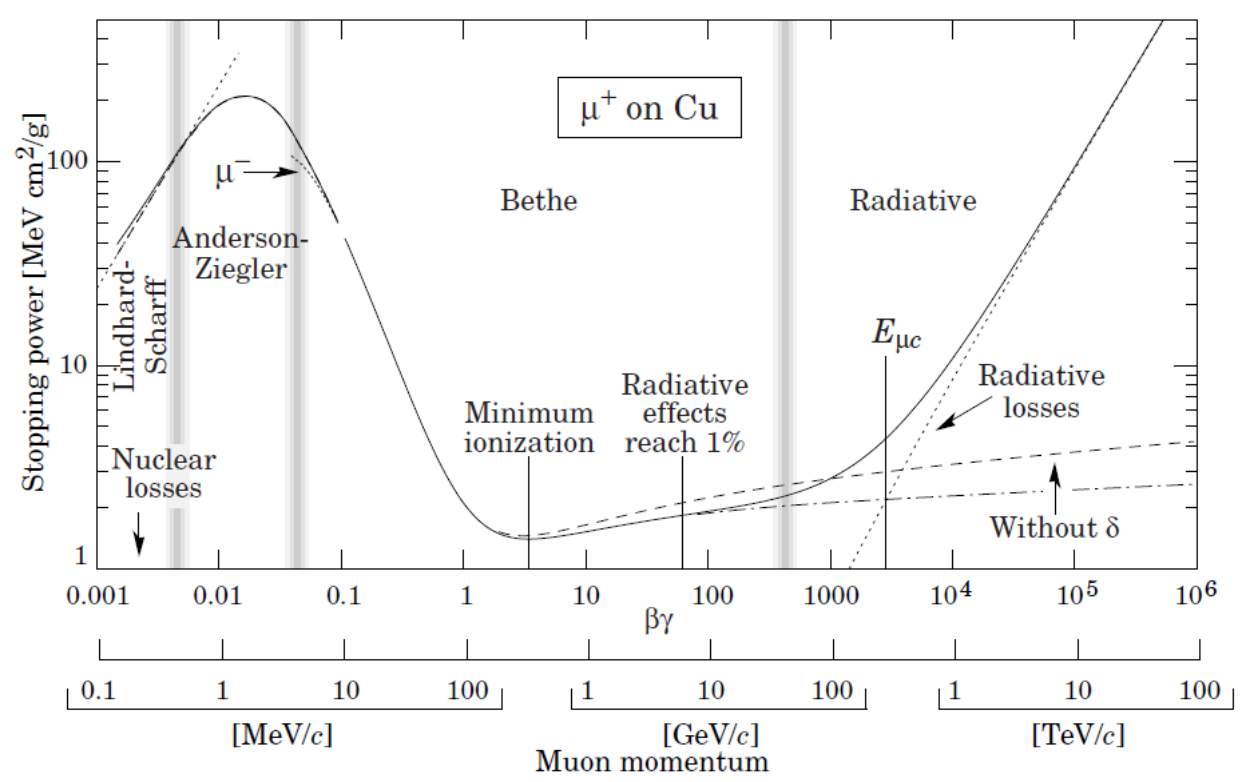

Figure 3.1: Stopping power $\left(=-\left\langle\frac{\mathrm{d} E}{\mathrm{~d} x}\right\rangle\right)$ for positive muons in copper as a function of $\beta \gamma=p / M c[6]$.

anism of energy loss for electrons at high energies. The energy loss by Bremsstrahlung is proportional to the energy $E$ of the incident particle and is given by

$$
-\left\langle\frac{\mathrm{d} E}{\mathrm{~d} x}\right\rangle_{\mathrm{rad}}=\frac{E}{X_{0}}
$$

The constant of proportionality depends only on the material and is called radiation length $X_{0}$. It describes the mean distance over which high energetic electrons lose $\frac{1}{e}$ of their energy via Bremsstrahlung alone. The mean free path due to pair creation $\lambda$ is proportional to the radiation length. To decrease the possibility of pair creation, it is necessary to keep the radiation length for tracking detectors as small as possible.

The Bethe-Bloch formula describes the mean energy loss $\mathrm{d} E$ in a material of thickness $\mathrm{d} x$. The energy loss of a particle in matter is a statistical process. For thick layers it is described by a Gaussian distribution with a mean value given by the Bethe-Bloch formula. For particles passing through only thin material layers $(\mathscr{O}(100 \mu \mathrm{m}))$ the energy loss is better described by a Landau distribution [26], because the number of collisions, while passing the material, is smaller. This distribution has a long tail towards high energy loss that is mainly caused by direct collisions with electrons. These $\delta$-electrons receive enough energy in the collision to ionise additional atoms. Because of the high-energy tail, the mean energy loss value is higher than the most probable value. 


\subsubsection{Photons in Matter}

The behaviour of photons in matter is different from the mechanisms described above for charged particles. Because they are not charged, there are no inelastic collisions with electrons. The three most important processes are the photoelectric effect, Compton scattering and pair production.

In the photoelectric effect and pair production the electron is absorbed and a beam of photons is attenuated while passing through matter. The intensity of a beam decreases exponentially with the thickness of the passed material $x$ as

$$
I(x)=I_{0} e^{-\mu x},
$$

with the initial intensity $I_{0}$ and the material-specific and energy-dependent mass attenuation coefficient $\mu$. During Compton scattering the photon is not absorbed, but the energy of the photon is changed.

The cross section of these processes depends on the photon energy and the material. If the photon energy is between the $\mathscr{O}(\mathrm{eV})$ and $\mathscr{O}(100 \mathrm{keV})$, the photoelectric effect is the most probable process. An electron is emitted by absorbing a photon, whose energy has to be larger than the binding energy of the electron. The remaining photon energy contributes to the kinetic energy of the electron. These low energetic photons are available in radioactive sources and laser set-ups. Because of their well known energy deposition they are used to characterise and test the properties of prototype modules for tracking devices.

At photon energies between $\mathscr{O}(100 \mathrm{keV})$ and $\mathscr{O}(10 \mathrm{MeV})$ the Compton effect is dominant. The photon scatters on a quasi-free electron and transfers a part of its energy to the electron. Neither the electron nor the photon provides information about the initial photon. For high energy photons, pair production is the most important process. If the photon energy is higher than twice the electron mass $(\mathscr{O}(\mathrm{MeV}))$, an electron-positron pair is produced. As mentioned above, the photon is absorbed and does not enter the calorimeter system. Only a reconstruction from the electron-positron pair is possible, but challenging.

\subsubsection{Multiple Scattering}

A charged particle passing material scatters elastically off the nuclei. This process, called multiple scattering, additionally influences the detector performance. The angular distribution of scattering processes is described by the Rutherford formula [27]. The total scattering angle $\theta$ after passing a material layer with the thickness $l$ is given by the sum of independent scatterings. It is approximated by a Gaussian distribution with a mean value of $0^{\circ}$. The standard deviation $\sigma_{\theta}$ of this distribution is given by

$$
\sigma_{\theta} \approx \frac{13,6 \mathrm{MeV}}{p v} \sqrt{\frac{l}{X_{0}}}
$$

with the momentum $p$ and velocity $v$ of the incident particle and the radiation length $X_{0}$. Especially for low momentum incident particles, multiple scattering limits the tracking precision. By reducing the material thickness and using material with higher radiation 
length, the standard deviation is decreased. For example, during testbeam measurements this effect has to be considered.

\subsection{Vertex Measurement}

The strategy for vertex measurement is to have finely segmented detector layers close to the interaction point. The data from the layers are analysed and pattern recognition and tracking programs transform the single points into particle tracks. Thus, the particle momentum is determined and in combination with other tracks the primary vertex as well as potential secondary vertices are identified. The spatial resolution of the vertex and the precision of the momentum measurements is influenced by the design of the tracking detector.

\subsubsection{Spatial Resolution}

The one-dimensional spatial resolution in one position is determined by the segmentation width of the sensitive element. Assuming a uniform particle occupancy, a binary readout and perfect module efficiency, the occupancy distribution in the segment is described by a rectangular function with the segment width $d$. The spatial resolution, given by the standard deviation $\sigma$, is the uncertainty on the position measurement calculated as

$$
\sigma=\frac{d}{\sqrt{12}}
$$

Effects by signal sharing between neighbouring segments and the threshold of the readout electronics are not considered.

\subsubsection{Vertex Resolution}

The vertex resolution depends on several detector parameters. Assuming two segmented detector layer at radii $r_{1}$ and $r_{2}$ with $r_{2}>r_{1}$ and a beam pipe wall at radius $r_{0}$, the vertex resolution is given by [28]

$$
\sigma_{\mathrm{vtx}}=\sqrt{\frac{r_{1}^{2}}{\left(r_{2}-r_{1}\right)^{2}} \sigma_{1}^{2}+\sigma_{2}^{2}+\left(2 r_{1}-r_{0}\right)^{2} \sigma_{\theta}^{2}} .
$$

To keep the vertex resolution small, a fine segmentation resulting in small one-dimensional spatial resolutions $\sigma_{1}$ and $\sigma_{2}$ is needed. Furthermore, multiple scattering in the beam pipe and the detector layers affects the vertex resolution. By having as little material as possible in the tracking detector the multiple scattering standard deviation $\sigma_{\theta}$ is reduced. In addition, a large lever arm of the detector (large $r_{2}-r_{1}$ ) and an inner layer as close as possible to the beam pipe (small $r_{1}$ and small $2 r_{1}-r_{0}$ ) improves the vertex resolution. These design criteria are considered while designing a vertex and tracking detector. 


\subsection{Signal Generation}

The basic operating principle of semiconductor detectors is the creation of electron-hole pairs due to the passage of ionising radiation. The charge carriers induce a signal, while drifting in an electric field towards the electrodes. Because of its properties and the good availability of silicon, a crystalline solid-state semiconductor, it is the commonly used material for semiconductor detectors.

\subsubsection{Fundamental Properties of Silicon}

In the energy-band theory, a material is described by introducing a valence band and a conduction band. Valence electrons are bound to individual atoms, whereas electrons in the conduction band move freely in the atomic lattice. In metal, these two bands overlap, which explains the good conductivity, whereas an insulator has a large gap between the bands, usually larger than $3 \mathrm{eV}$. The band gap of a semiconductor is usually smaller than $3 \mathrm{eV}$ and thus atoms are ionised with a small amount of energy leading to mobile ("free") charge carriers in the sensor.

Silicon is an indirect semiconductor and the average energy required to lift an electron to the conduction band is $I_{0}=3.62 \mathrm{eV}$. This energy is about 10 times smaller than the energy required for gas ionisation [29]. The radiation length of silicon is $21.82 \mathrm{~g} \mathrm{~cm}^{-2}$ and the density is $2.329 \mathrm{~g} \mathrm{~cm}^{-3}$ [6].

With the density $\rho$ and the average energy for electron-hole creation $I_{0}$ of silicon, the signal charge $Q$ of a MIP is calculated as

$$
Q=\frac{\left\langle\frac{\mathrm{d} E}{\mathrm{~d} x}\right\rangle \rho d}{I_{0}} .
$$

Assuming a sensor with a thickness of $d=250 \mu \mathrm{m}$, the average generated charge is $Q=24000$ e. The current induced by the electron-hole pairs created by a MIP is much smaller than the one induced by thermal charge carriers. At room temperature ( $T=300 \mathrm{~K}$ ) the density of intrinsic free charge carriers is $9.65 \cdot 10^{9} \mathrm{~cm}^{-3}$ [30]. In a pixel with a size of $50 \mu \mathrm{m} \times 400 \mu \mathrm{m}$, about $5 \cdot 10^{7}$ free electrons exist. Thus, pure silicon is not suitable as sensor material.

\subsection{2 pn-Junction}

A semiconductor is doped to modulate its electrical properties. Doping intentionally inserts impurities into the material. Silicon, which is a Group IV element, is doped with either Group V elements to add an extra valence electron (donor) or Group III elements, which are missing the fourth valence electron (acceptor).

A donor atom introduces states close to the conduction band. Electrons in these states are easily excited to become free electrons. In contrast, acceptor atoms provide holes, which are unoccupied states in the valence band. Semiconductors doped with donor impurities are called n-type, whereas those doped with acceptor impurities are known as p-type. The names indicate the type of the majority charge carriers.

Typical doping concentrations are in the range between $10^{13} \mathrm{~cm}^{-3}$ and $10^{18} \mathrm{~cm}^{-3}$, whereas 
the density of intrinsic free charge carriers is as already mentioned $9.65 \cdot 10^{9} \mathrm{~cm}^{-3}$. Hence, in contrast to pure silicon, in doped silicon, the additional electrons and holes become the most important charge carriers.

A combination of n-type and p-type silicon is used to produce a sensor with very low leakage current and low free charge carrier concentration. At the emerging pn-junction the majority charge carriers diffuse into the oppositely doped side due to the concentration difference. They recombine with the other charge carriers. An electrical field caused by the remaining space charge compensates the diffusion process. An equilibrium configuration is reached and the pn-junction region is depleted of free charge carriers.

The width of the depletion zone $d$ and thus the number of electron-hole pairs contributing to the signal is increased by applying a bias voltage $U_{\text {bias }}$. It is calculated by [31]

$$
d=\sqrt{\frac{2 \epsilon \epsilon_{0}}{N_{\mathrm{D}} e} U_{\text {bias }}}
$$

with the donor concentration $N_{\mathrm{D}}$. Ideally, the width of the depletion zone stretches over the whole sensor material. The bias voltage needed to achieve this state is referred to as depletion voltage.

By applying the depletion voltage, the sensor material is depleted of free charge carriers. But because of thermal generation of charge carriers, a volume current occurs, which is highly dependent on the temperature. This leakage current is a source for shot noise in the readout electronics.

If the bias voltage is too high a junction break-down can happen. It is characterised by a huge increase of the leakage current. There are multiple possible sources for a break-down: thermal instability, tunnelling and avalanche multiplication.

\subsubsection{Charge Collection}

The movement of free charge carriers in a semiconductor is caused by drift and diffusion. Diffusion is a random thermal movement. In the presence of a concentration gradient the charge carriers diffuse into areas with a lower charge carrier concentration. Charge collection via diffusion is possible, but because of its random-walk nature the charge collection time is longer compared to drift collection.

In the presence of an electrical field, charge carriers are separated and drift to the electrodes. While drifting the charge carriers induce immediately a current signal on the electrodes described by Ramo's theorem [32]. This signal collection via drift results in an instantaneous induced signal and a fast collection time.

In most tracking detectors a magnetic field is present in addition to the electrical field. Because of the Lorentz force the charge carriers that drift towards the electrodes are deflected. The resulting drift direction differs by the Lorentz angle from the original direction. Consequently, a magnetic field leads to a systematic shift of the detected position. This deviation is compensated in most particle detectors by inclining the detector with respect to the nominal particle incidence. 


\subsubsection{Radiation Damage in the Sensor Material}

Silicon detectors suffer from radiation damage, which is caused by the high particle flux as for example at the LHC. Non-ionising interactions of incident particles with silicon atoms change the properties of the sensor material. The displacement of atoms modifies the silicon crystal structure. Point defects occur like vacancies, which are missing atoms in the lattice, interstitials, which are extra silicon atoms, and substitutionals, meaning an impurity atom on a silicon lattice site. Furthermore, more complex defects, like cluster defects, are produced if enough energy was transferred to the recoil atoms. Figure 3.2 shows a schematic view of some possible defects.

Because of the defects additional localised states emerge in the band gap. Depending on the position of these energy levels sensor properties are influenced. The main effects of radiation damage caused by bulk defects are an increase of the leakage current, a reduction of the signal height and a change in the effective doping concentration. In the following, the origin for these detector effects is briefly summarised. A more detailed description can be found in [33].

Radiation damage caused by different particles varies in the resulting effects and hence it is difficult to compare. To scale the radiation damage to a standard irradiation, the Non-Ionising Energy Loss (NIEL) [34] hypothesis is used. The basic assumption of the hypothesis is that any change in the material caused by radiation damage scales linearly with the amount of energy deposited in the material. The NIEL hypothesis works very well to scale the radiation damage of any particle to the damage of $1 \mathrm{MeV}$ neutrons.

A defect in the middle between valance band and conduction band increases the thermal generation rate of charge carriers. The defect acts as a rest stop, which divides the band gap in two smaller energy steps. A bound electron is thermally excited to the defect centre and then excited to the conduction band. A free electron-hole pair is created, which causes an increase of the leakage current.

Recombination of electron-hole pairs is a well known process, which decreases the signal

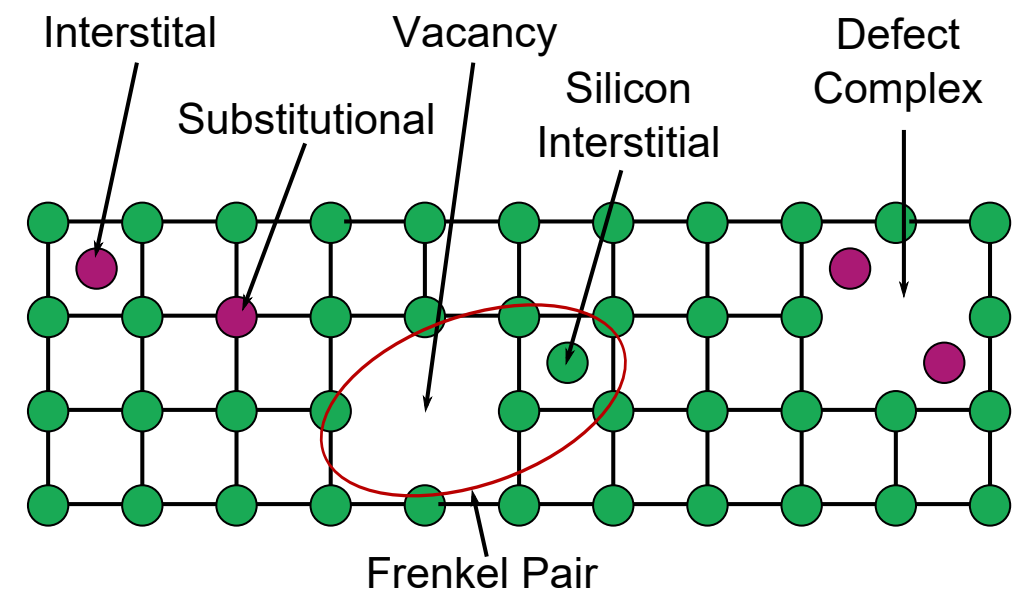

Figure 3.2: Possible defects that occur in a crystal lattice. 
amplitude. Defect centres capture charge carriers of opposite sign and consequently increase the recombination rate. The life time of a charge carrier decreases as well as the signal size. In addition, radiation induced defects act as scattering centres, which reduce the mobility of the charge carriers. As a consequence, the drift velocity and the induced current decrease and again a smaller signal is measured. Moreover, defect centres temporary trap charge carriers at a typically shallow level. Trapped charge carriers are released with a delay of the order of $\mu$, which is longer than the readout time of the sensor. Thus, these charges are not detected in time and the signal amplitude is reduced.

The compensation of donors or acceptors by radiation-induced centres is an effect resulting in a changing doping concentration. It is more probable to compensate donor states than acceptor states. For example, in n-type material free electrons are compensated by radiation-induced acceptors resulting in a reduced concentration of negative charge carriers. The removal of charge carriers leads to a type inversion. The effective doping concentration changes from positive to negative, converting an n-type doped material into a material, which has acceptor-like states similar to a p-type material. This change has a direct influence on the depletion depth and the voltage needed for full depletion. For the innermost layer of the present ATLAS Pixel Detector the type inversion took place in 2012 [35]. Figure 3.3 shows the mean value of the effective depletion voltage as a function of the fluence. At a fluence of about $2 \times 10^{13} n_{\mathrm{eq}} / \mathrm{cm}^{2}$ the type inversion takes place, which can be seen in the vanishing depletion voltage.

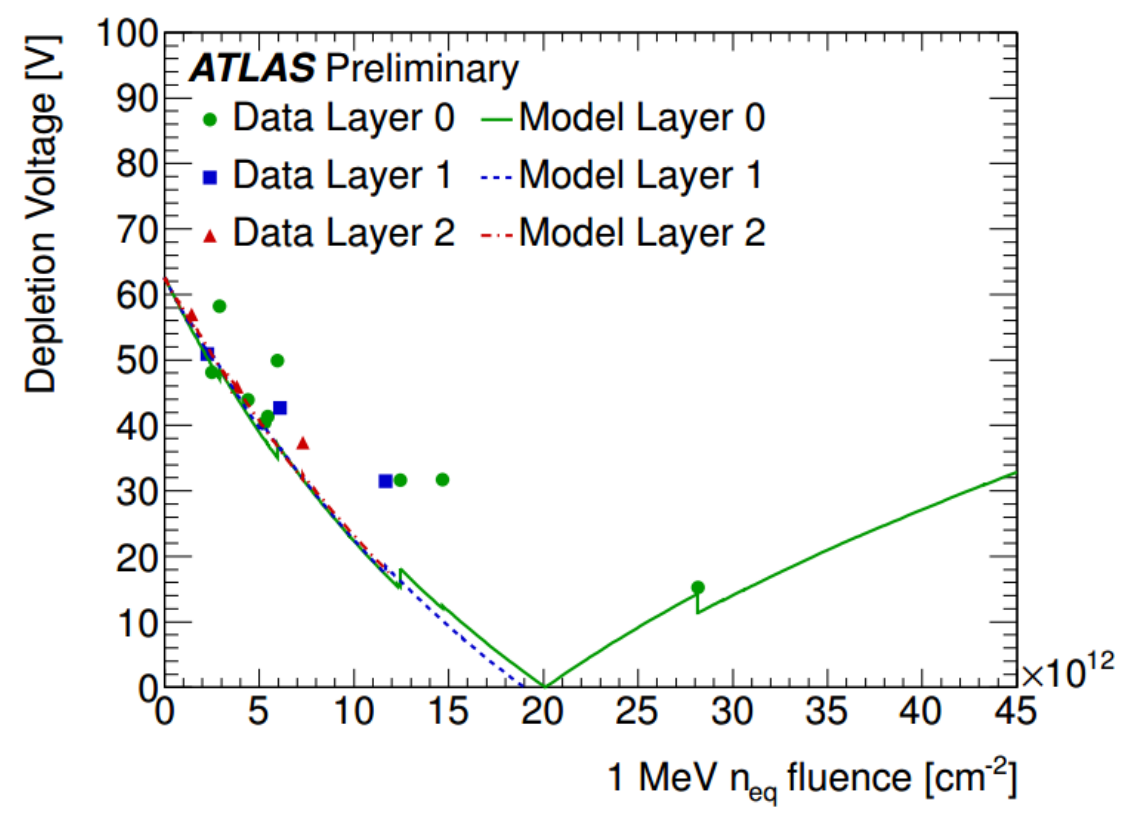

Figure 3.3: Mean value of the effective depletion voltage as a function of the $1 \mathrm{MeV}$ neutron equivalent fluence shown for all three ATLAS Pixel Detector layers. Model predictions for each layer are superimposed [35]. 
In summary, the presence of defects caused by radiation leads to higher leakage currents and a reduced signal. An increase of the noise is one result of a larger leakage current. Together with a smaller signal, the signal to noise ratio decreases significantly. By cooling the detector modules, the thermal generation of charge carriers is reduced and consequently, the leakage current is decreased. In addition, various sensor designs like thinner sensor thickness and 3D electrode geometries are used for detector upgrades, to prevent a reduced signal due to trapping in high radiation environments.

\subsection{Readout Electronics}

The last section described the signal charge generation in a sensor. The readout electronics handles the measurement of this signal charge and the signal processing. Usually, the readout electronics consists of an analogue part for signal amplification and shaping and a digital processing logic. The direct measurement of the charge signal is typically realised with a charge-sensitive amplifier (CSA). The signal charge is collected on a feedback capacitor, which needs to be reset after the signal from the sensor is processed. As feedback circuitry a constant current source is added in parallel to the feedback capacitor to obtain an approximately linear falling edge. The CSA signal is often required to be further amplified or shaped.

The number of channels in a vertex detector is so large that a digitisation is required, turning the analogue information into a bit stream. One example of an analogue to digital conversion is a discriminator. The amplifier output signal is compared to a threshold voltage. As it can be seen in Figure 3.4, the discriminator output is on the logic high level as long as the amplifier output signal is larger than the threshold voltage. The time between the rising and the falling edge of the discriminator output is referenced as timeover-threshold (ToT). The ToT is in first order proportional to the charge at the input of the CSA if its output signal has a triangular shape with a constant linear falling edge. If the shape of the output signal differs from the described shape, the relation between
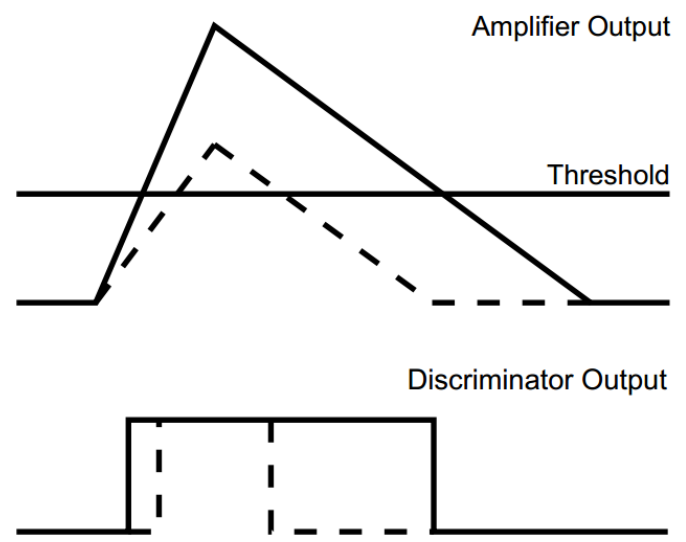

Figure 3.4: Amplifier output and discriminator response for a small (dashed line) and a large (solid line) charge signal on the amplifier input. 
ToT and charge is more complex than linear. The signal amplitude and the ToT are not connected via the intercept theorem any more.

Taking a closer look at Figure 3.4, a discrepancy in the discriminator rise times is noted for the different signal amplitudes of the amplifier output. This time walk is caused by the finite amplifier rise-time. A hit with a low charge is detected too late and not associated with the correct trigger, if the time walk is too large. Consequently, the time walk needs to be kept as low as possible.

The discriminator decision limits the data for further processing to only signals with a hit reported. This process is called zero-suppression.

\subsubsection{Noise in Analogue Readout Electronics}

In the analogue part of the readout electronics the three main sources of noise need to be considered and minimised. Shot noise is a consequence of the discrete nature of electric charge and the statistical fluctuation of the number of charge carriers that is linked to it. The leakage current of the sensor produces shot noise at the input of the amplifier. Thermal noise is caused by the random thermal motion of charge carriers. It is proportional to the temperature and appears at all frequencies. Low-frequency (or $1 / f$ ) noise occurs in almost all electronic devices and results from a variety of effects. Unlike shot and thermal noise the $1 / f$ noise depends on the frequency.

Usually, the noise is quoted as equivalent noise charge (ENC), which relates the noise to a charge that is needed on the readout input to create an output signal of the same amplitude. Not all electronics components are perfectly identical in a pixel detector, e.g. due to fluctuations in the production (layer thickness, geometrical size, etc.). Small differences in the pixels threshold are caused and consequently a dispersion of the threshold distribution of the pixels originates. The threshold dispersion yields a similar effect as the noise sources described above. The noise of a pixel detector module is defined as the quadratic sum of the ENC and the dispersion. The threshold-to-noise ratio specifies the possibility to measure hits caused by noise, which is a key measure.

\subsubsection{Radiation Damage in the Readout Electronics}

Readout electronics suffers from radiation damage caused by ionisation, but not from bulk damages like the sensor material, which have a non-ionising nature. The active devices in complementary metal-oxide-semiconductor (CMOS) logic have a much higher doping concentration and are not influenced by the changes in the effective doping concentration. Furthermore, a semiconductor and an insulator differ in the width of the band gap and the material structure is different. A semiconductor has a regular crystal structure, whereas the oxide structure is irregular. Consequently, the displacement of atoms due to irradiation damage has no effect in the CMOS logic.

Surface damages close to the semiconductor-insulator interface (e.g. $\mathrm{Si}^{-\mathrm{SiO}_{2}}$ ) are of importance. This ionising energy loss is caused by any type of radiation, because it depends primarily on the absorbed energy. Irradiation damage is proportional to the energy absorption per unit volume, the so-called dose. 


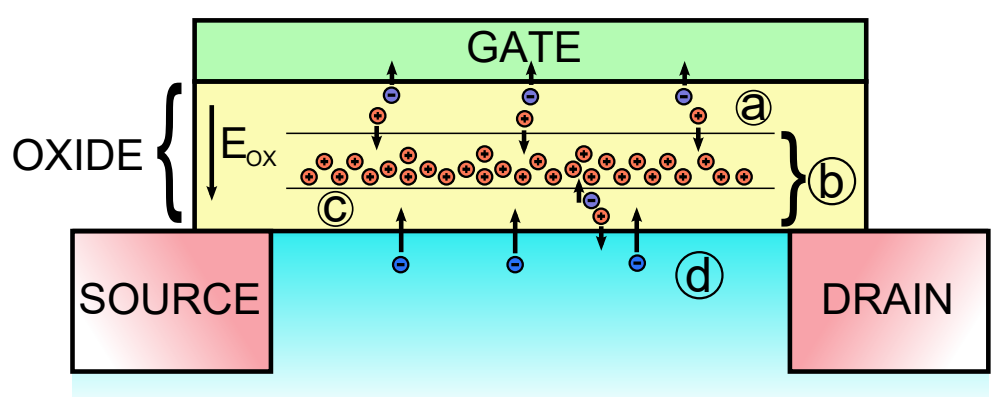

Figure 3.5: Schematic cross-section of a MOS transistor and enlarged the processes in the oxide layer. a) Electron-hole pairs are formed by radiation. b) Holes are trapped. c) Traps are cleared by tunnelling electrons from Si. d) Electrons are injected from Si. The figure is adapted from [36].

Figure 3.5 shows a schematic cross-section of a MOS transistor and enlarged the processes in the oxide layer. The ionising radiation creates charge carriers in the interface region that diffuse or drift to other locations. The electrons move several orders of magnitude faster than the holes and leave the insulator, whereas the holes move slower towards the $\mathrm{Si}_{-} \mathrm{SiO}_{2}$-interface. In regions with a higher defect density trapping is more likely. A fraction of the holes is captured in traps, which are deep because of the large band gap of the oxide. Trapped holes in the few nm close to the silicon are rapidly cleared by electrons tunnelling from the silicon. The trapped holes change the concentration of charge and modify the field distribution. The result is a shift in the gate-source voltage and the threshold voltage of the transistor changes. The operating point of the transistor is modified, which influences the whole readout electronics.

To decrease the effects by surface radiation damage, transistors with very thin oxide layers are used. Because the number of holes available for trapping decreases with the oxide thickness, thin layers reduce the trapping possibility. Furthermore, the region that is free of trapped holes due to tunnelling is used. 



\section{Pixel Detector Technologies}

Many different pixel detector technologies exist for particle tracking. The decision for a certain technology is based on requirements, which the detector environment defines, like the high particle flux at the LHC. Furthermore, specific detector functionalities are required, such as a high detector granularity and fast readout electronics to identify the signatures characteristic of the physics processes.

The current ATLAS Pixel Detector is a so-called hybrid detector. It consists, in contrast to a monolithic pixel detector, of a pixellated sensor and a readout chip, which are separate entities, connected pixel-by-pixel via bump bonds. With this hybrid concept, the two components can be developed separately of each other and different technologies can be used for the tasks of signal generation and processing. The sensor and the readout chip of the current ATLAS Pixel Detector and the Insertable B-Layer will be explained in the following.

With new challenges that come with the upgrade towards the HL-LHC, new detector technologies are investigated. Four-chip modules, which cover an area of approximately $16 \mathrm{~cm}^{2}$, and their characteristics are described in this chapter. Larger modules require less handling steps during assembly, which drive the costs per area.

A new pixel detector technology is a hybrid module with an active high-voltage CMOS sensor, which is produced in an industrial process and aims for a cost effective production. The concept is introduced at the end of this chapter.

\subsection{Current ATLAS Pixel Detector}

The ATLAS Pixel Detector [20,37] is the innermost part of the ATLAS Experiment. It plays a major role in fulfilling the detector performance requirements (see Section 2.3.1), which drive the design choices. The Pixel Detector consists of three barrel layers at radii of $50.5 \mathrm{~mm}, 88.5 \mathrm{~mm}$ and $122.5 \mathrm{~mm}$, and three endcap discs per side (see Figure 4.1). Therefore, it provides two-dimensional hit information for at least three points per charged track. The full pseudorapidity range of $|\eta|<2.5$ is covered by the tracker. The minimum radius for the innermost layer of $50.5 \mathrm{~mm}$ is set because of practical limitations by the beam pipe vacuum system. The nominal pixel size is $50 \times 400 \mu \mathrm{m}^{2}$, which on the one hand satisfies the requirements arising from the high particle flux and on the other hand are set by the electronics design limitations. With this pixel size, the maximum occupancy is approximately $5 \cdot 10^{-4}$ per pixel area and a proper readout is ensured.

The basic units of the ATLAS Pixel Detector are 1744 modules of silicon sensors and readout chips. The roughly $2 \times 6 \mathrm{~cm}^{2}$ large silicon sensors are connected to 16 readout chips, which are arranged in two rows. Every sensor pixel is connected to a readout pixel using a bump bond. The total active area is approximately $1.7 \mathrm{~m}^{2}$ and the total number of pixels is approximately $8 \cdot 10^{7}$. To handle the distribution of commands and collection 


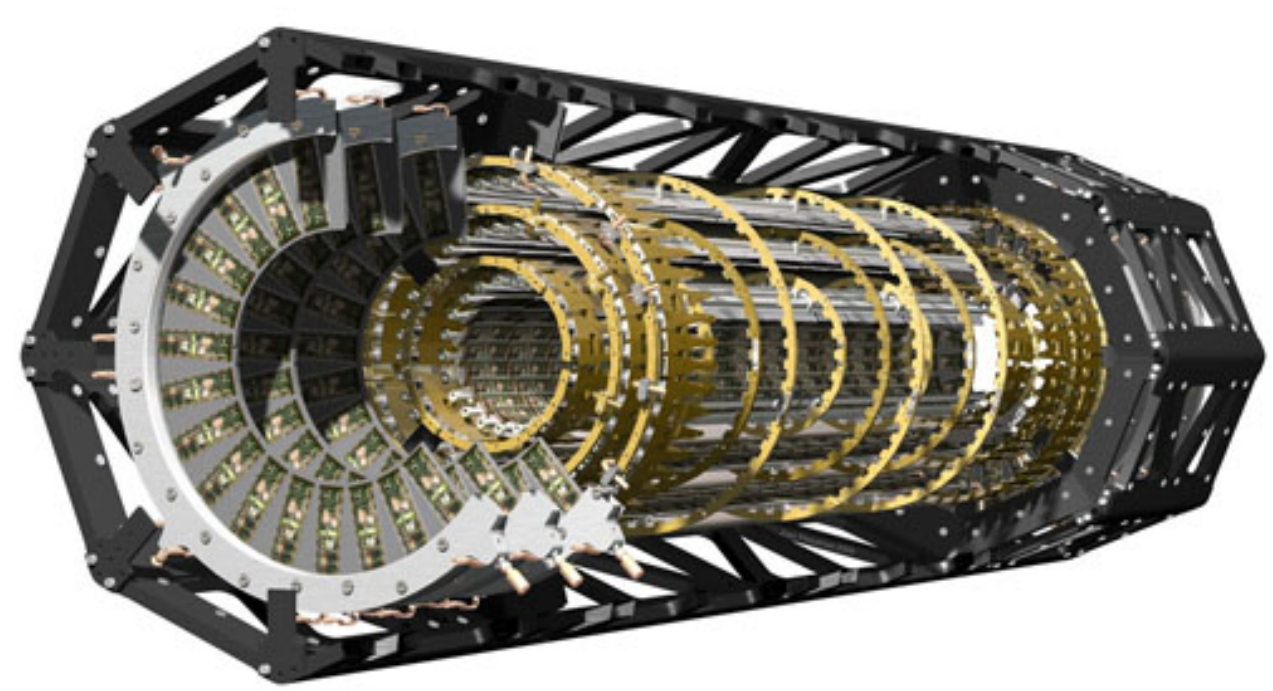

Figure 4.1: Cut-away view of the ATLAS Pixel Detector showing barrel layers and endcap discs including the support structures.

of data for all 16 readout chips, each module is equipped with a Module Control Chip. To keep the material budget low, the readout chips and the Module Control Chip are thinned down to thicknesses below $200 \mu \mathrm{m}$. The total material budget of the barrel is approx. $11 \% X_{0}$ for $\eta=0$ and under normal incidence, of which ca. $3.5 \% X_{0}$ come from each barrel layer, the rest is caused by supports and services. The carbon support structures hold the modules and are tilted by $20^{\circ}$ with respect to the radial direction to compensate for the Lorentz angle caused by the magnetic field. The modules are cooled to $-7^{\circ} \mathrm{C}$ by cooling pipes.

\subsubsection{Sensor}

The silicon sensors used for the ATLAS Pixel Detector are $250 \mu \mathrm{m}$ thick with $\mathrm{n}^{+}$-doped pixels in an n-type bulk material. The bias voltage is connected to the p-doped electrode on the back side, which is surrounded by a multi-guard ring structure (see Figure 4.2). This structure locates the main voltage drop on the sensor side opposite to the bump connection and protects the front-end chip from discharges.

The n-in-n design fulfils the requirement of high charge collection efficiency even after a fluence of $10^{15} n_{\mathrm{eq}} / \mathrm{cm}^{2}$, which corresponds to the expected lifetime dose. Radiation damage causes type inversion (see Section 3.3.4) and gradually increases the depletion voltage. For unirradiated sensors the depletion zone starts to grow from the sensor backside, which is p-type doped. The pixels are not isolated from each other until full depletion (see Figure 4.2). After type inversion the n-in-n design allows the detector to operate with a good charge collection efficiency, because the depleted region propagates from the $\mathrm{n}^{+}$-pixel side towards the p-implantation. The pixels are insulated from each other, even if the sensor is only partly depleted. This operation mode is especially important if the maximum sensor bias voltage is reached. 

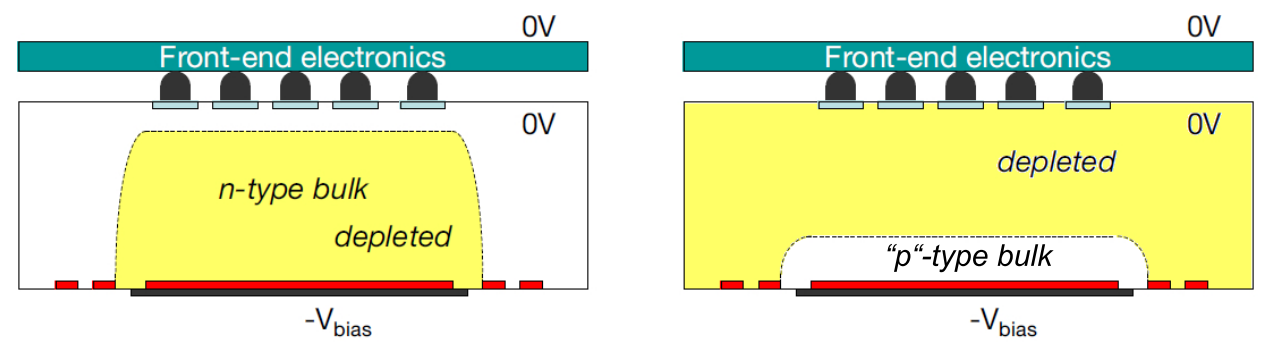

Figure 4.2: Development of the depletion zone in an $n$-in- $n$ sensor before (left) and after (right) type inversion. The depletion zone grows from different sides of the sensor. Before type inversion from the p-side, afterwards from the n-pixel side, which allows operation even if the sensor is only partly depleted [37].

For the sensor production, diffusion oxygenated float zone silicon is used, because it has an increased radiation tolerance compared with standard silicon [38]. Consequently, it has an improved charge collection after type inversion and lower depletion voltage, which is important considering the maximum allowed sensor bias voltage of $-600 \mathrm{~V}$.

\subsubsection{Readout Chip}

The readout chip of the ATLAS Pixel Detector is the Front-End I3 (FE-I3) [39], which is implemented in the $250 \mathrm{~nm}$ CMOS process at IBM. Measurements show good signal to noise performance even after $500 \mathrm{kGy}$, which is the expected dose for the innermost layer after approximately five years of LHC operation.

The FE-I3 is designed to digitise the charge signal received from the sensor pixel. The 2880 readout cells, one per sensor pixel, are arranged in 18 columns and 160 rows. The chip size is $7.2 \times 10.8 \mathrm{~mm}^{2}$, of which $75 \%$ are active area and the rest is inactive periphery. Each readout cell has an analogue block and a digital readout part operating at a $40 \mathrm{MHz}$ clock. The analogue part of a readout cell contains a high-gain, fast preamplifier followed by a second amplifier stage and a differential discriminator. The digital readout part transfers the hit pixel address, a hit time stamp and a charge information to the chip periphery.

The parameters of the analogue components are tuned to ensure a uniform response of all pixels from a given charge. The most important digital to analogue converter (DAC) parameters are the discriminator threshold and feedback current. The threshold is set globally using the 5-bit DAC (GDAC) and individually per pixel with the 7-bit trim DAC (TDAC). The value of the feedback current is globally adjusted using the 8-bit IF DAC and individually using the 3-bit feedback DAC (FDAC). The individual adjustment is necessary to minimise pixel to pixel variations.

The implemented readout electronics provides an approximately linear relation between the charge of the detected signal and the ToT. The charge information can be used to increase the spatial resolution in case of charge sharing between two or more pixels. The 8-bit ToT information and the pixel address is temporarily stored in the digital circuitry until further processing. 


\subsection{Insertable B-Layer}

The Insertable B-Layer (IBL) [40] is a fourth pixel layer, which was added to the existing ATLAS Pixel Detector in 2014 (see Figure 4.3). It is located at a radius of $33.4 \mathrm{~mm}$ between

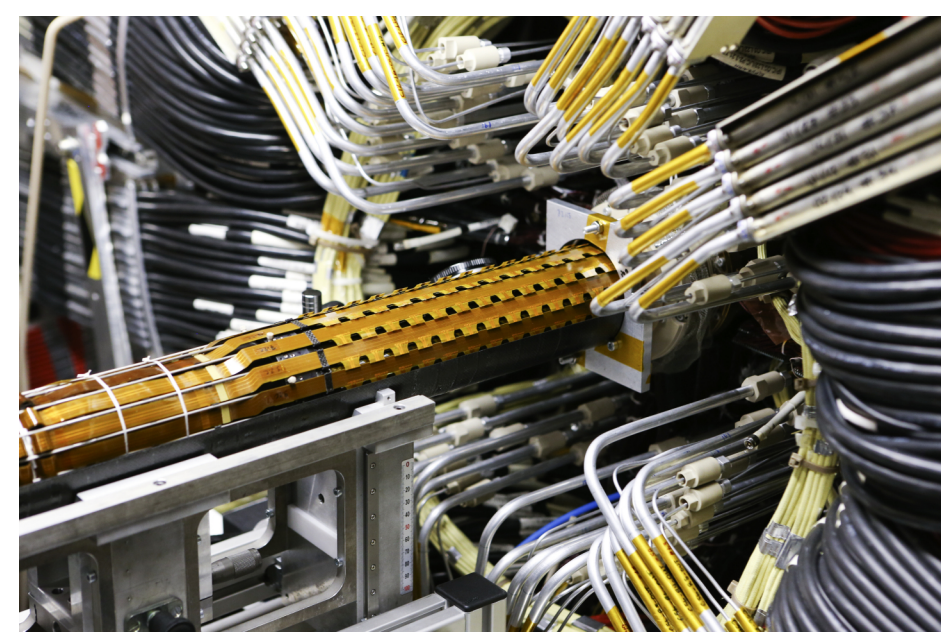

Figure 4.3: Photograph of the IBL installation into the Inner Detector of the ATLAS Experiment [41].

a new beam pipe with a smaller radius and the current inner pixel layer. The main motivation of the IBL is to provide a robust tracking performance as the instantaneous luminosity of the LHC increases and the radiation damage compromises the performance of the inner pixel layers. The small IBL radius improves the vertex resolution and the b-tagging performance.

At the same time, the location close to the interaction point required more radiation hard sensors and readout electronics, which need to withstand with fluences of up to $5 \times 10^{15} n_{\mathrm{eq}} / \mathrm{cm}^{2}$ and $2.5 \mathrm{MGy}$ total ionising dose, respectively. To cope with the high occupancy, the pixel size was reduced to $50 \times 250 \mu \mathrm{m}^{2}$ and a more efficient readout chip was necessary. The available space between the new beam pipe and the inner pixel layer does not allow module overlaps in the direction along the beam. Thus, geometrical inefficiencies of the modules needed to be reduced. In the radial direction the modules are inclined by $14^{\circ}$.

The IBL comprises two module layouts, which use the same readout chip, the Front-End I4B. They are bump-bonded to two sensor technologies that differ in the readout electrode geometry: planar and 3D electrodes. The roughly $2 \times 4 \mathrm{~cm}^{2}$ silicon sensors with planar electrodes are connected to two readout chips, forming double-chip modules. These 168 modules are located in the middle of the barrel and represent $75 \%$ of the total area. The other $25 \%$ of the IBL area are covered with 122 silicon sensors with 3D electrodes, which are connected to only one readout chip, so-called single-chip modules. They are placed at the outer barrel regions. The total area of the IBL is approximately $0.18 \mathrm{~m}^{2}$ with a total number of approximately $12 \cdot 10^{6}$ pixels.

To optimise the tracking and vertexing performance, the material budget of the IBL was minimised. The thickness of the sensors and the readout chips was reduced and support 
structures of low density carbon foam were used. The radiation length is below $2 \% X_{0}$ for perpendicular traversing tracks. Evaporative cooling is used to cool the modules to $-15^{\circ} \mathrm{C}$.

\subsubsection{Sensor}

As mentioned above, two different sensor technologies are used for the IBL. The socalled planar modules are $200 \mu \mathrm{m}$ thick and have planar $\mathrm{n}^{+}$-doped implants in an n-type bulk of diffusion oxygenated float zone silicon. They are similar to the sensors of the Pixel Detector. To reduce geometrical inefficiencies, the outermost pixels are extended to $500 \mu \mathrm{m}$ and a new guard ring structure was introduced [42]. The number of guard rings was reduced to 13 and the innermost eleven rings are placed below the edge pixel implants (see Figure 4.4). Consequently, the inactive edges were reduced to less than $450 \mu \mathrm{m}$ to match the design requirements. Studies showed that the charge collection efficiency is above the required $97 \%$ after the IBL fluence, since the voltage limit for IBL was increased to $-1000 \mathrm{~V}[40]$.

The 3D silicon sensors are $230 \mu \mathrm{m}$ thick and have a p-type bulk material. Pillars are etched orthogonal to the surface into the bulk and filled with doped silicon to form electrodes. This design decouples sensor thickness and charge collection distance and increases the radiation hardness. Figure 4.5 shows details of the 3D sensor layout from the two manufacturers. The main difference between the layouts is the column depth.

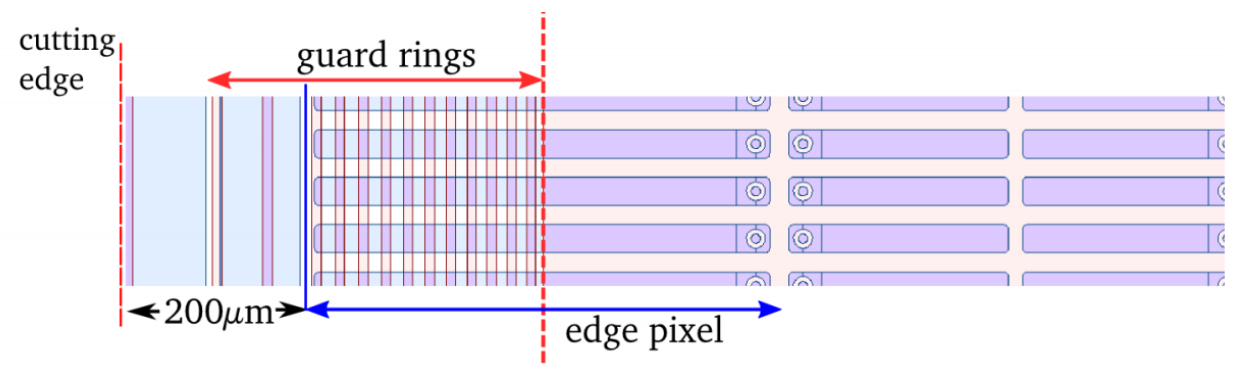

Figure 4.4: Schematic drawing guard ring structure of the IBL planar sensor. The guard rings are located on the opposite side of the pixel implant. They are shifted below the edge pixel [43].
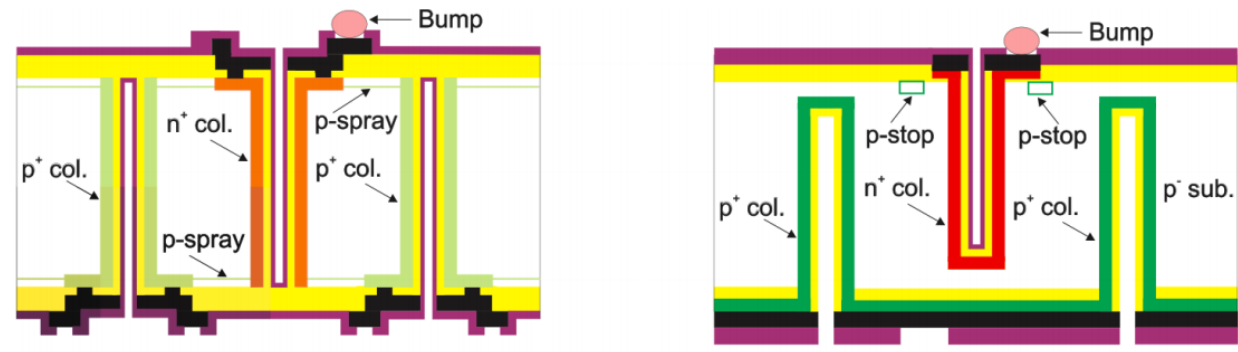

Figure 4.5: Schematic drawing of the IBL 3D pixel sensor designs of two manufacturers [43]. 
Because of the short distance between the bias and the charge collecting electrode $(\approx$ $75 \mu \mathrm{m})$ the depletion voltage after irradiation is below $-200 \mathrm{~V}$. The production of 3D design sensors needs non-standard processing, which results in higher costs and a lower yield. The problem of irradiation-induced defects is still present, although the depletion voltage and thus leakage current is significantly reduced.

\subsubsection{Readout chip}

The readout chip for the IBL is the Front-End I4 (FE-I4), which has a feature size of $130 \mathrm{~nm}$. This CMOS technology improves the radiation tolerance because of the thin gate oxide transistors. Thus, the FE-I4 fulfils the requirement to withstand 2.5 MGy total ionising dose. The new readout chip has an area of $20.2 \times 18.8 \mathrm{~mm}^{2}$, which is five times the area of the FE-I3 (see Figure 4.6), and the active area covers $90 \%$ of the chip. The pixel array

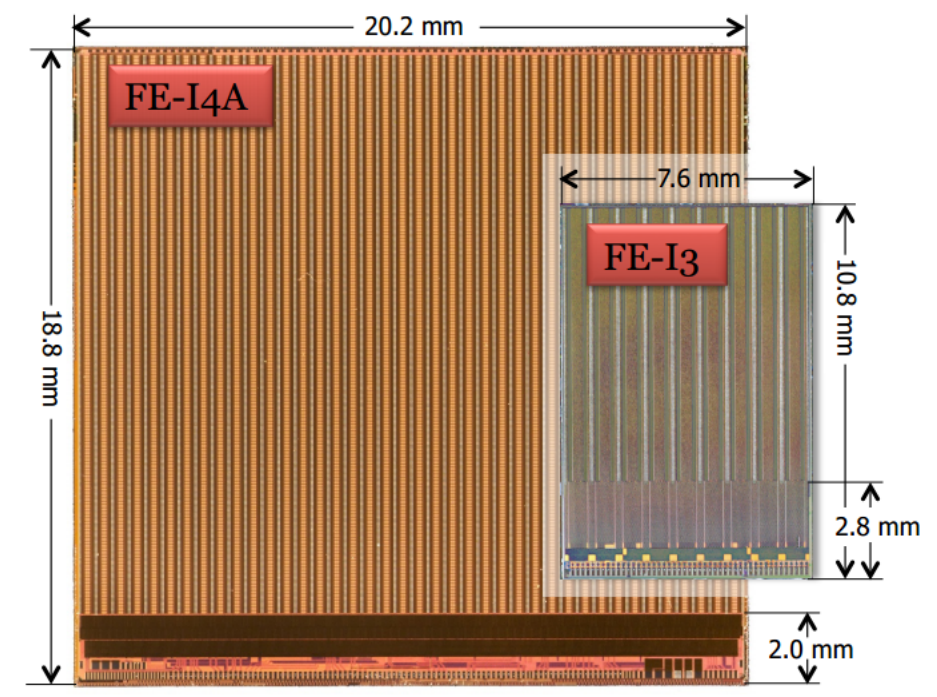

Figure 4.6: Picture of the FE-14 and the FE-I3 to compare the sizes [43].

consists of $80 \times 336$ pixels with an analogue and a digital circuitry. The analogue part is comparable to the FE-I3. Both readout chips employ a two-stage amplifier, but in contrast to the FE-I3, the second stage of the FE-I4 amplifier is AC coupled to the first stage.

The digital architecture was improved to cope with the high particle rates. Instead of a column drain readout, placing the trigger logic and data storage in the periphery of the chip, the FE-I4 has a four-pixel digital region, which stores the data locally until the trigger decision. The readout speed was increased to up to $160 \mathrm{MHz}$.

Figure 4.7 sketches the analogue pixel logic. The amplifier has two stages, a fast preamplifier and an AC-coupled second amplifier. The preamplifier integrates the induced charge of the sensor using a feedback capacitor, which is discharged by a constant feedback current. The global feedback current is set by the 8-bit DAC PrmpVbpf. For in-pixel adjustment of the feedback current the 4-bit DAC, the so-called FDAC, is used. Subsequently, the discriminator compares the input signal with the adjustable threshold. The threshold can be controlled by the temperature compensated DAC, Vthin_Alt, which is composed 


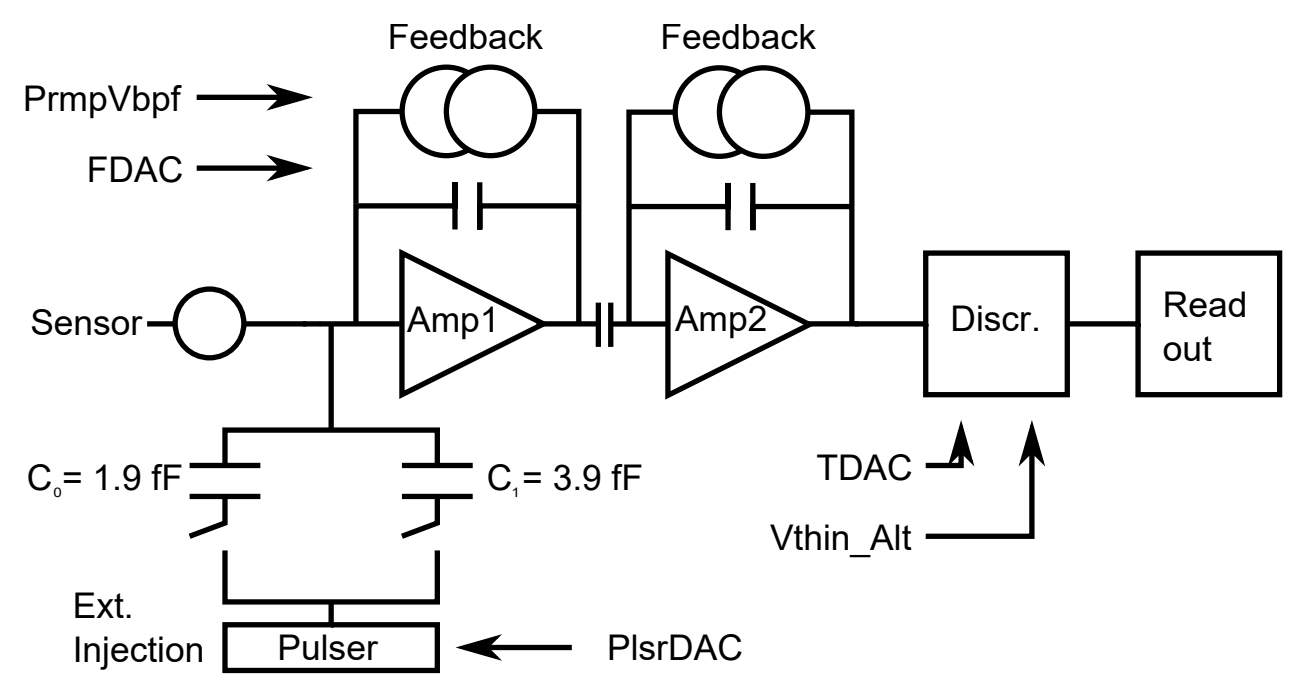

Figure 4.7: Schematic view of the analogue pixel cell. Signals are shaped by a twostage amplifier, digitised by a discriminator and then passed on to the digital readout logic. The most important configuration parameters are named.

of two 8-bit values for coarse and fine adjustment. Besides the global control, there is a 5-bit in-pixel adjustment named TDAC. The ToT information was reduced to 4-bit with respect to FE-I3.

To test the readout chip, an external charge injection mechanism is implemented in the chip architecture. The charge is generated by applying a voltage pulse to different capacitors. The amplitude is controlled by a 10-bit PlsrDAC value. Two capacitors can be selected $C_{0}$ and $C_{1}$, which have design values of approximately $1.9 \mathrm{fF}$ and $3.9 \mathrm{fF}$, respectively.

The FE-I4 chip is able read out a time window of $16 \times 25 \mathrm{~ns}$. When an external trigger is received, the chip generates up to 16 consecutive internal triggers, each with a length of $25 \mathrm{~ns}$. The resulting timing distribution is referred to as Level 1 (Lvl1) distribution.

\subsection{Four-Chip Modules}

As described in Section 2.4.4, the detector requirements for the new ITk for the HL-LHC depend on the distance to the interaction point. The large area of the outer layers is one of the challenges and it drives the module design criteria. The fluence and dose will be comparable to the IBL environment. Thus, the technologies used for the IBL are investigated for feasibility in the ITk.

The readout chip for IBL, the FE-I4B, was tested to withstand 2.5 MGy total ionising dose and hence, it is usable for the outer layers of ITk. To cover an area of several square metres, larger sensors are investigated, connected to several readout chips. These fourchip modules cover an area of approximately $4 \times 4 \mathrm{~cm}^{2}$ and the sensor is read out by four FE-I4B chips. Larger modules have the advantage of fewer handling steps during production, which is reduces the costs. 
A first step towards a four-chip module is the usage of two double-chip modules as one pseudo four-chip module. Figure 4.8a shows a wafer, which contains two double-chip modules. Normally, they would be diced out as two separate modules. To obtain a pseudo four-chip module, they are cut out as one large sensor. As shown in Figure 4.8b, the

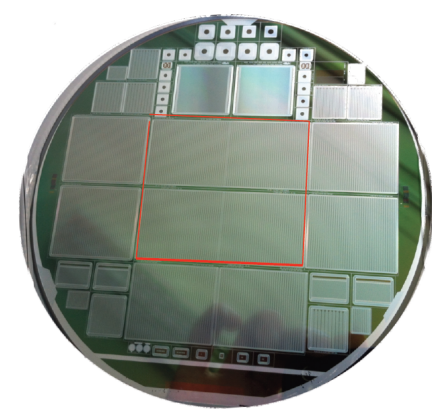

(a) Picture of a 4" silicon wafer. The red square indicates the pseudo four-chip module.

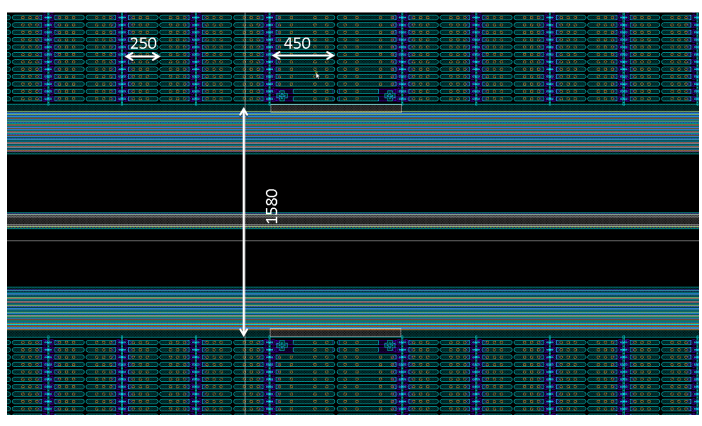

(b) Schematic drawing of the pixel layout between the two double-chip modules.

Figure 4.8: Geometry of the pseudo four-chip module [44].

distance between the two double-chip modules is approximately $1.6 \mathrm{~mm}$, which is an inactive area in the middle of the pseudo four-chip module. The standard pixel dimension is $250 \times 50 \mu \mathrm{m}^{2}$ and the two pixel columns between the two readout chips of one doublechip sensor are $450 \mu \mathrm{m}$ long. The active area of the four-chip module is $40.4 \times 34.0 \mathrm{~mm}^{2}$ and it is a planar n-in-p sensor design.

$\mathrm{N}$-in-p sensors have a p-type bulk material, which does not suffer from type inversion. Furthermore, it contains $\mathrm{n}^{+}$-implants on the pixel side, which are isolated from each other even at low depletion voltages. From the beginning of operation, the depletion zone grows from the pixel side towards the back side implant and not only after type inversion (compare with 4.1.1). On the back side of the sensor, there is one large p-type implantation so that the sensor only needs one structured side, which potentially reduces the processing costs. Besides this advantage in processing, the performance of single-chip modules with an n-in-p sensor is similar to ones with an n-in-n sensor [45].

The production of the pseudo four-chip module is part of a collaboration between the Max Planck Institute for Physics and the University of Göttingen. During this thesis, the characteristics of an unirradiated module were measured. In lab measurements, the current-voltage characteristic was measured, shown in Figure 4.9. The leakage current of the module was investigated down to $-150 \mathrm{~V}$. Down to this voltage no breakdown was observed. The module was operated at $10^{\circ} \mathrm{C}$, because the four configured readout chips produce heat that increases the leakage current and noise.

Furthermore, the discriminator thresholds of the readout chips were determined (for a detailed description of the tests see Section 5.1.2). Figure 4.10 shows the threshold distri- 


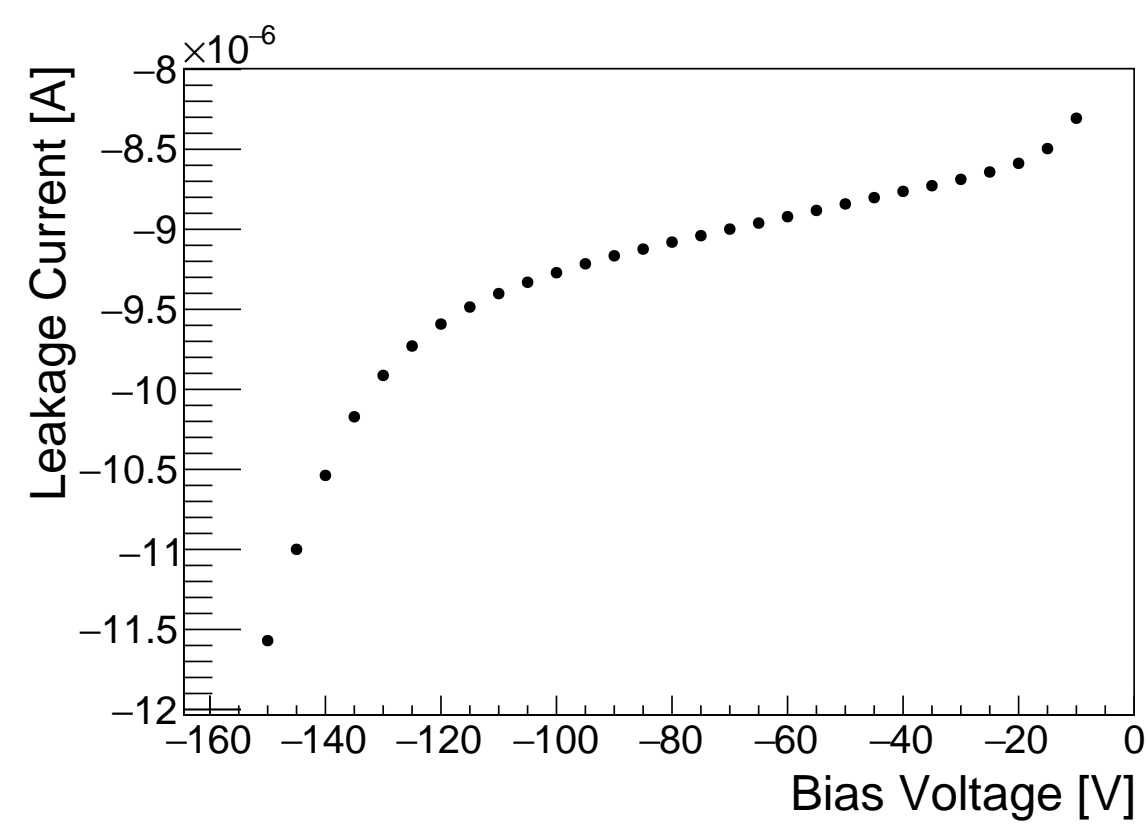

Figure 4.9: Current-voltage characteristic for an unirradiated pseudo four-chip module. The error bars are too small to be seen.

bution for the pseudo four-chip module, which was tuned to 2000e. The dispersion among the pixels is approximately 50e, showing that the tuning succeeded to yield a uniform discriminator threshold for all pixels. The equivalent noise charge of the module is shown in Figure $4.10 \mathrm{~b}$ and is approximately $140 e$. The threshold-to-noise ratio is 13.4 , which yields a small possibility to measure hits caused by noise. These values are comparable to the single-chip modules.

In measurements with a radioactive source the bump bond quality of the module was investigated. Strontium-90, which is a $\beta^{-}$-source emitting electrons, was used. The module was triggered using a scintillator, which was located below the assembly. Only high energetic electrons were able to pass the sample and reach the scintillator, causing an energy loss in the sensor comparable to that of a MIPs. In the measurement $10^{7}$ external triggers were collected. Considering the number of pixels, this number of triggers is required so that every pixel registers approximately 100 hits. This calculation assumes a homogeneous irradiation of all four chips and does not take the solid angle of the point-shaped source into account. The hit map of the source scan can be seen in Figure 4.11. The beam spot of the source is clearly visible. The pixels in between the double-chip modules have more hits due to their larger size as expected.

In Figure 4.12a the distribution of the clustered ToT is plotted, in which clustered means that the ToT values of all pixels that belong to the same incident particle are summed up. This clustered ToT corresponds to the collected charge. The histogram was fitted with a convolution of a Gaussian and a Landau distribution (see Section 3.1.1). The most probable value is at a ToT of approximately 6 . The module was tuned to respond with 

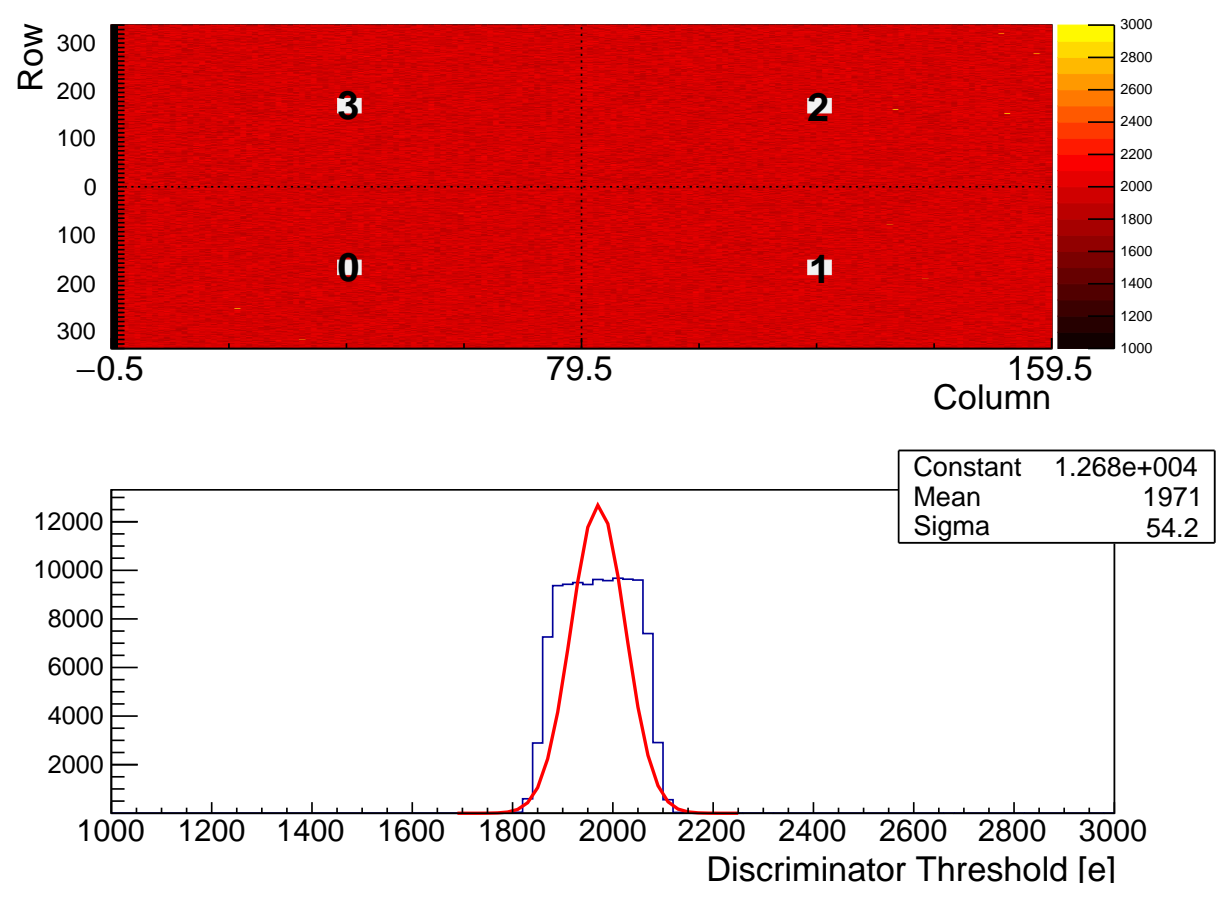

(a) Threshold.

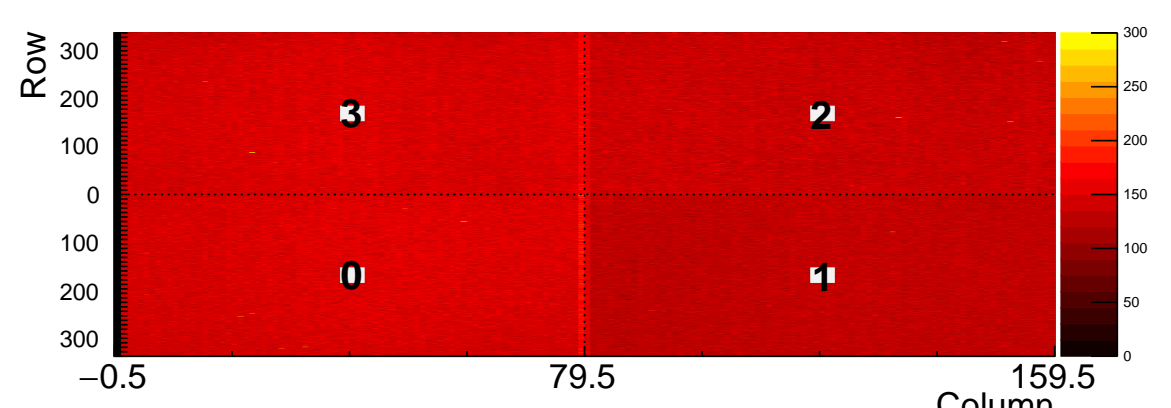

Column

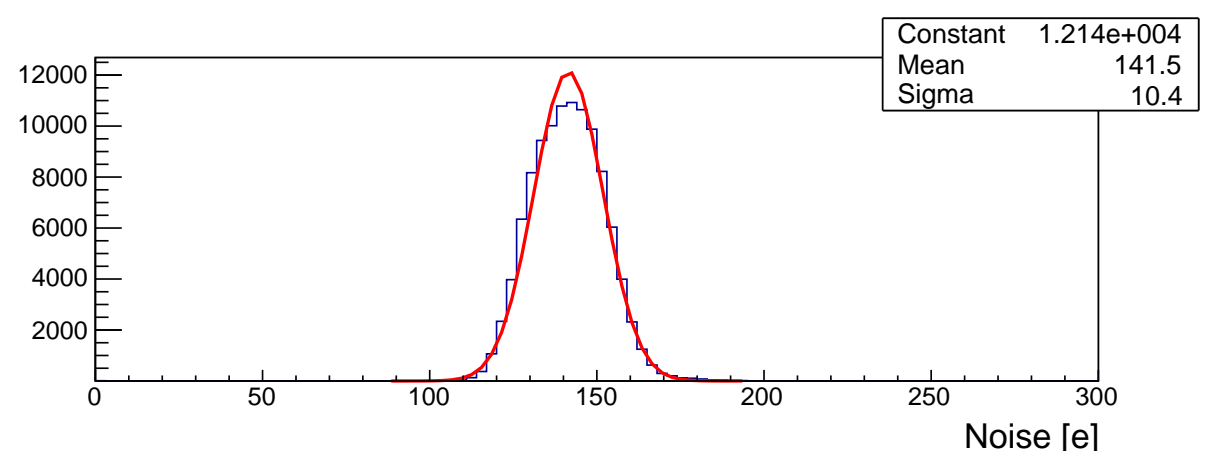

(b) Equivalent Noise Charge.

Figure 4.10: Distribution of the discriminator threshold after a tuning to $2000 e$ with a pseudo four-chip module. The top plots show a pixel map and the bottom plots all pixel values in one histogram. 


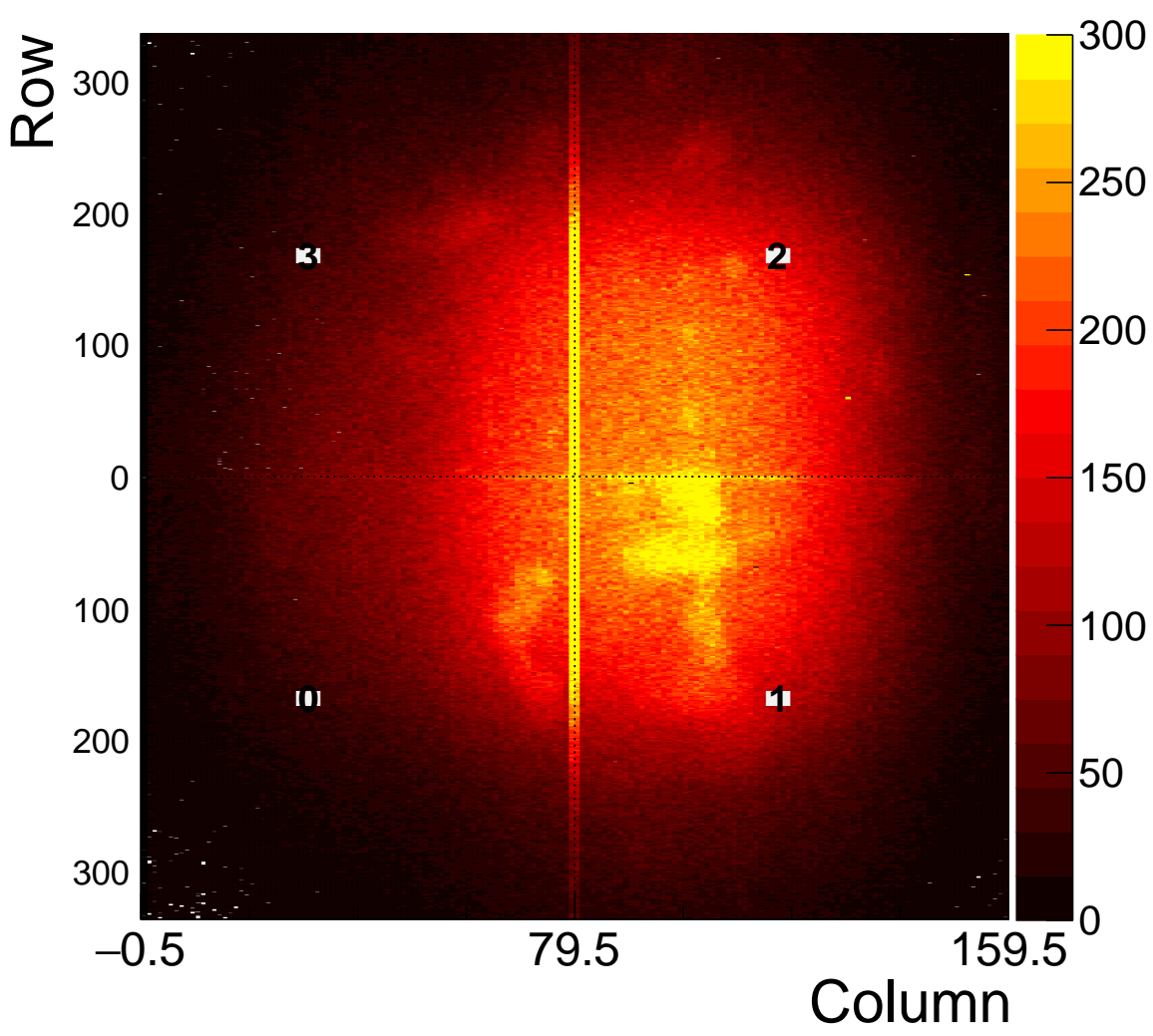

Figure 4.11: Hit map of the pseudo four-chip module after a measurement with a Sr-90 source. The beam spot is visible and the larger pixels have more hits.

a ToT of 7 to a deposited charge of 14000 e, which is the charge produced by a MIP in $200 \mu \mathrm{m}$ of silicon. Thus, the ToT response is close to the expected value.

The Lvl1 distribution is shown in Figure 4.12b. The distribution around the target value is caused by the readout clock jitter and time walk effects. The few entries, which are found in all bins, are caused by noise hits not correlated to the external trigger signals. Approximately 60 pixels were disabled for the measurement, because they were noisy and showed hits even without a source. The statistics at the edges of the module is so low that in this scan a few pixels have no hits. Less than $0.1 \%$ of all pixels have no proper bump-bond connection. Thus, the bump-bond quality is very good. Bump bonding of a $200 \mu \mathrm{m}$ thick sensor to four readout chips is possible with good quality.

In addition, the depletion voltage of the sensor was measured, using the Strontium-90 source. The energy loss of the electrons depends on the passed, depleted length in the sensor (see Section 3.1.1). The depletion zone grows with the square root of the applied bias voltage (see Section 3.3.2). Figure 4.13 shows the sensor bias voltage as a function of the squared clustered ToT, which translates into the collected charge. In turn, the collected charge is proportional to the width of the depletion zone. If the ToT saturates, the whole 


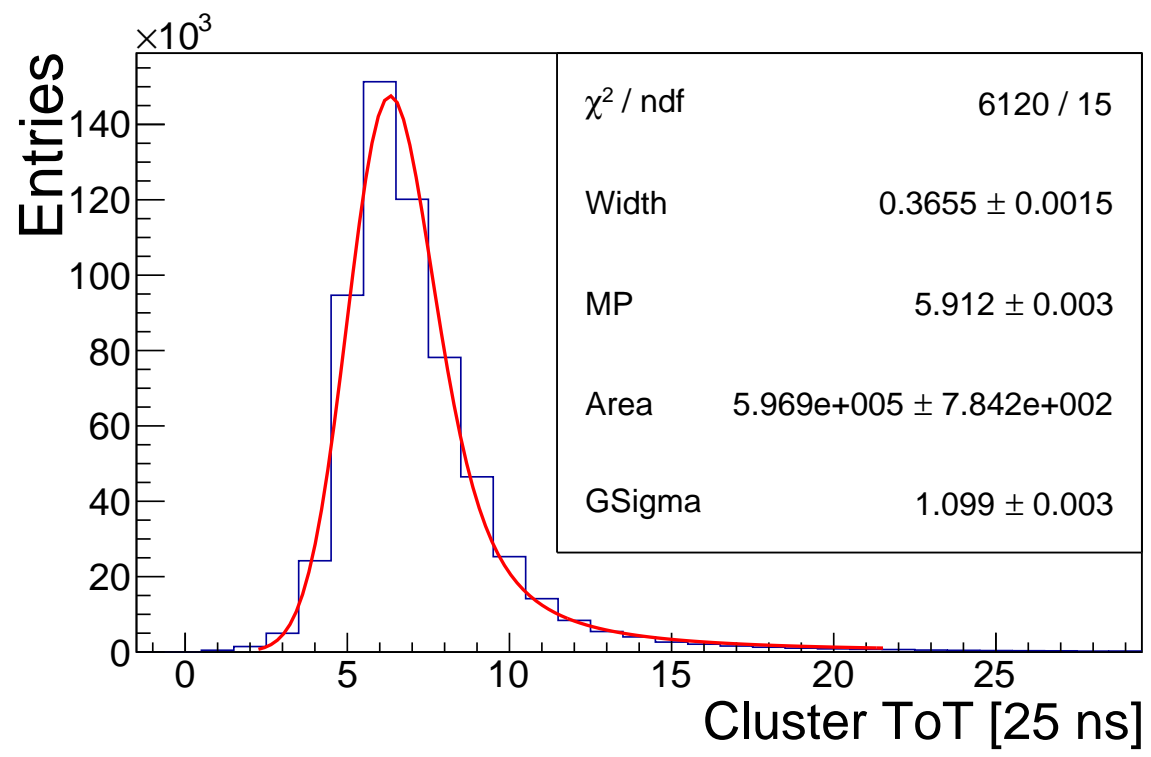

(a) ToT.

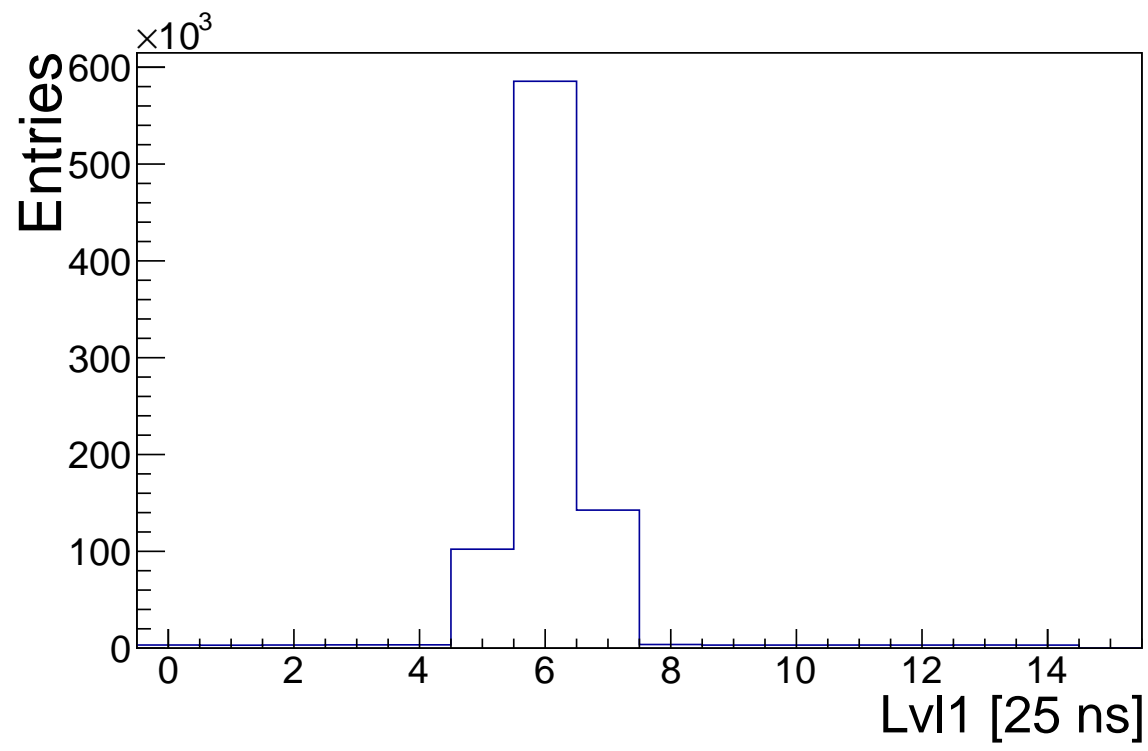

(b) $L v / 1$.

Figure 4.12: $T O T$ and $L v / 1$ distribution of a source measurement with Strontium-90. 


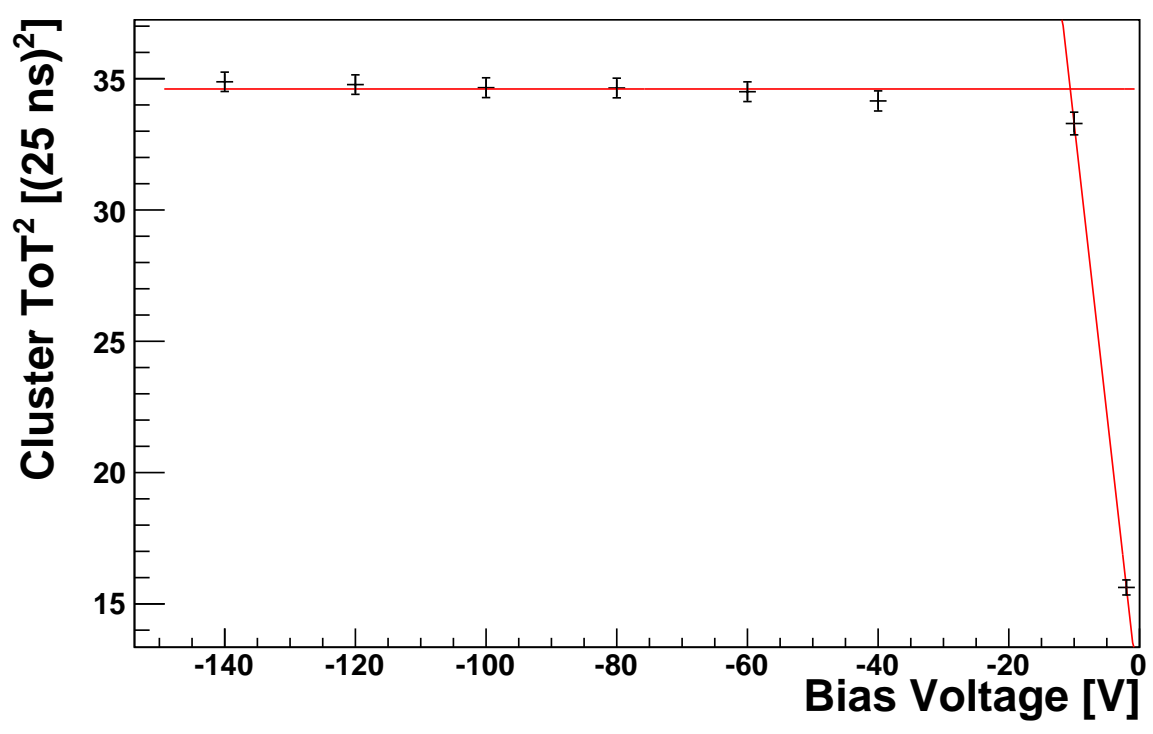

Figure 4.13: Squared clustered TOT as a function of the sensor bias voltage for the pseudo four-chip module. From the intersection of the constant and the linear function the depletion voltage was found to be approximately $-11 \mathrm{~V}$. The pixel dispersion is the stated errorbar.

sensor is depleted. Figure 4.13 was fitted with a constant function for the saturated region and a linear function in the region, in which the depletion zone grows. The intersection of these two functions is defined as the depletion voltage. For the investigated pseudo four-chip module, the depletion voltage was found to be approximately $-11 \mathrm{~V}$.

The hit efficiency was measured in test beam measurement with $4 \mathrm{GeV}$ electrons at DESY together with the Max Planck Institute for Physics. The detailed results are presented in [46]. The hit efficiency for the pseudo four-chip module was found to be above $98 \%$.

\subsection{Active High-Voltage CMOS Sensors}

Besides hybrid module concepts based on well-known passive sensor designs, like the four-chip modules (see Section 4.3), new approaches are considered for the outer layers of ITk. The large area requires cost effective detectors. Active high-voltage CMOS sensors are investigated for the usage in ITk, integrated in hybrid modules.

The CMOS sensor technology has been successfully developed for more than 20 years, but in Monolithic Active Pixel Sensors (MAPS), as they are used in cameras. In contrast to a hybrid detector, a MAPS detector combines sensor and signal processing with inpixel electronics in one silicon wafer. In a low-doped epitaxial layer the charge collection happens mainly through diffusion. Thus, MAPS detectors are used in low rate and low radiation environments like the STAR Experiment [47] at Relativistic Heavy Ion Collider in Brookhaven. 


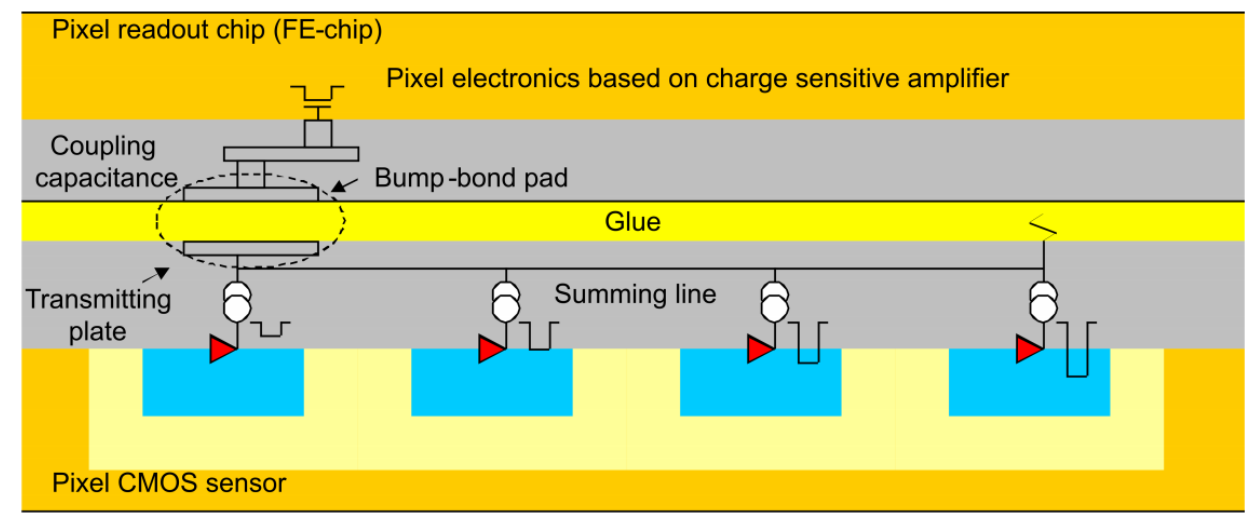

Figure 4.14: Pixel cross-section of a capacitively coupled pixel detector with a hybrid detector [51]. The readout chip (top) is glued to an active CMOS pixel sensor (bottom).

To use active CMOS sensors in a high rate and high radiation environment, like the HL-LHC, a new high-voltage CMOS technology [48] is used. The high-voltage technology allows a combination of standard low-voltage CMOS transistors, which are used to implement internal electronics, and the bias of the sensor with more than $-50 \mathrm{~V}$. Consequently, a depleted zone of about $10-100 \mu \mathrm{m}$ is formed, depending on the CMOS process and the bulk resistivity. The charges generated by ionizing particles in the depleted area are collected by drift to allow a fast read out that fulfils the HL-LHC requirements.

The high-voltage CMOS technology is available in a large variety of CMOS processes, which are industrially available from several vendors. The industrialised production is an advantage with regard to a cost effective detector. Sensor productions on larger wafers with sizes of 8 " or even 12 " are available and industrial machinery could be exploited to process and handle the wafers. Active CMOS sensors can reduce the costs per square centimetre of the sensor by a factor of two [49]. Together with more cost effective interconnections, like wafer to wafer bonding or gluing techniques, active high-voltage CMOS pixel sensors are a promising candidate for future use.

For the future operation in the ATLAS ITk, the present hybrid pixel detector is the baseline design. The current passive sensor is replaced with a capacitively coupled active CMOS sensor [50] (see Figure 4.14). The usage of a dedicated readout chip makes more complex electronics possible. Because of the first amplification stage in the sensor the coupling to the readout chip can be capacitive. The sensor is glued to the readout chip and a complex bump bond connection is not necessary. The total hybrid module costs could be decreased by a factor four [49].

The CPIX demonstrator programme was started to demonstrate the suitability of commercial high-voltage CMOS technologies for ITk. A number of prototypes with different layouts and using different technologies from several foundries are characterised to evaluate the usability. In this thesis the HV2FEI4v2 [51] is studied, which is implemented 


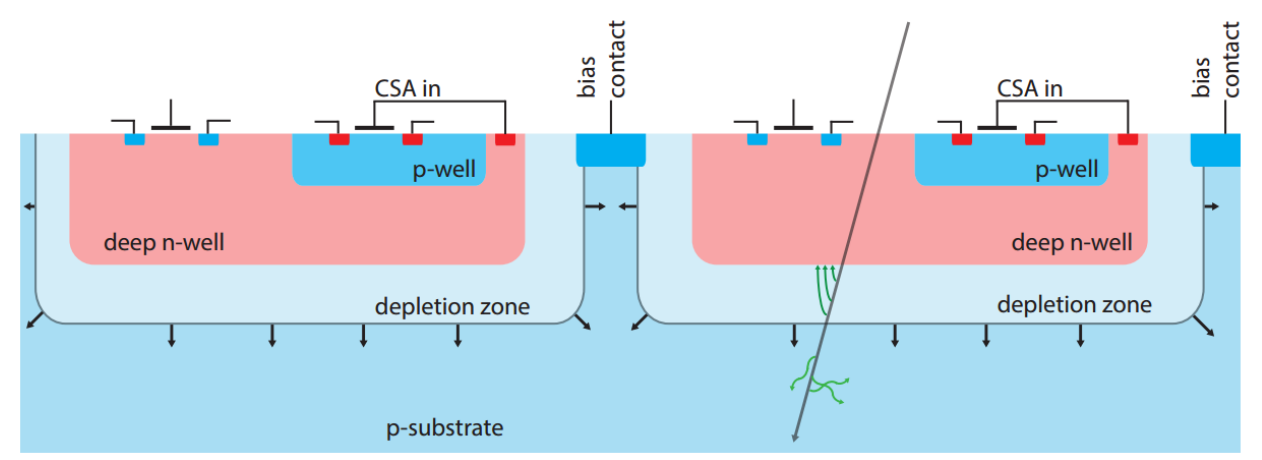

Figure 4.15: Schematic cross-section of an active high-voltage CMOS sensor with the multiple-well structure [52]. For details see text.

in the Austria Microsystems (AMS) $180 \mathrm{~nm}$ high-voltage CMOS process. It is glued to the FE-I4 readout chip (see Section 4.2.2), building a capacitively coupled pixel detector (CCPD).

A schematic cross section of an active high-voltage CMOS sensor is shown in Figure 4.15. It is based on the multiple-well structure, which houses the CMOS electronics. The deep $\mathrm{n}$-well in a p-substrate, which has a resistivity of above $10 \Omega \mathrm{cm}$, is used as the chargecollecting electrode. If a reversed bias voltage of about $-60 \mathrm{~V}$ is applied, the depth of the depletion zone is about $15 \mu \mathrm{m}$. The PMOS transistors are implemented directly inside the deep n-well, whereas the NMOS transistors are located in p-wells that are embedded in the deep n-well.

The CMOS electronics in the sensor build a charge-sensitive amplifier, a comparator with the possibility of pixel-by-pixel threshold adjustment and an output stage. The transistor parameters are set by a global and a pixel shift register. In addition to the command lines, the active sensor needs three supply voltages and four tunable reference voltages to set the transistor working points.

The HV2FEI4v2 sensor prototype has a size of about $2.2 \times 4.4 \mathrm{~mm}^{2}$, which is much smaller than the FE-I4 (see Figure 4.16). The sensor pixels are arranged in 240 unit cells,

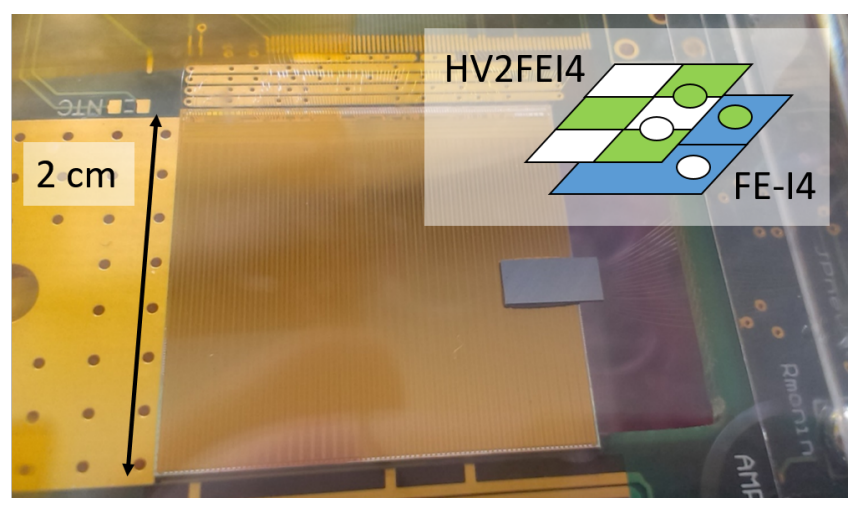

Figure 4.16: Picture of HV2FEI4V2 sensor glued to an FE-14 readout chip. The inset shows schematic drawing of the subpixel mapping for one unit cell. 
each containing six pixels. Three sensor pixels are connected to one FE-I4 readout chip pixel. The schematic drawing in the upper right corner of Figure 4.16 shows the subpixel mapping for one unit cell. Details concerning the subpixel encoding will be explained in Section 6.4 . 


\section{Readout System Integration and Measurement Set-Up}

\subsection{USBpix Readout System}

In order to perform characterisation measurements with prototype modules, the compact USBpix system [53,54] is used. It is an existing readout system for FE-I3 and FE-I4 readout chips. The hardware components are modular providing large flexibility. Figure 5.1 shows the USBpix components: the Multi-IO board and the available adapter cards. These components are described in the following.

The Multi-IO board (see Figure 5.1a) is a multi-purpose Field Programmable Gate Array (FPGA) card. This digital interface board holds a microcontroller, which controls the data transfer of the USBpix system. The USB connection to the host PC is established via the USB 2.0 interface. The FPGA handles the histogramming of the data, holds the trigger logic and manages the clocking of the module. The on-board memory for histogramming and buffering is a 2 MByte static random-access memory (SRAM).

Different adapter cards were designed to connect the USBpix Multi-IO board to specific detector modules providing electronic functionality dedicated to the module type. The adapter card to connect FE-I4 single chip modules (see Figure 5.1b) handles logic level shifting and conversion and the powering of the module.

To establish a simultaneous connection to up to four FE-I4 chips, the Burn-In Adapter Card (see Figure 5.1c) is used. The readout chips share the same clock and command outputs and parallel readout is possible [55]. Thus, the Burn-In Adapter Card is used to read out four-chip modules as one big module.

The General Purpose Adapter Card (see Figure 5.1d) extends the digital in- and output capabilities of the Multi-IO board. It has analogue blocks including four power supply channels, four voltage and twelve current source channels and an injection pulse generator with programmable voltage levels. In addition, level shifters for CMOS signals and lowvoltage differential signalling transmitters are available. All analogue settings and voltage and current readback are controlled via the I2C interface of the Multi-IO board.

The USBpix readout system is the standard system for Insertable B-layer (IBL) prototype module tests. Operation of FE-I4 chips is fully implemented and well tested. Furthermore, the USBpix readout system is completely integrated with the EUDET/AIDA telescope [56] including the Trigger Logic Unit (TLU), which allows test beam measurements (see Section 5.3). 


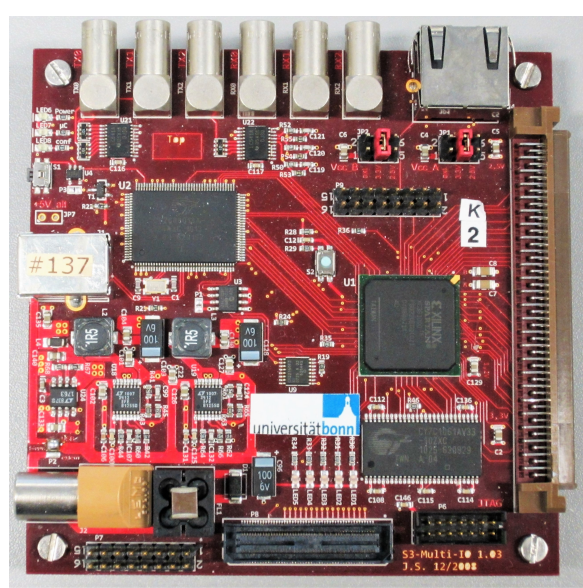

(a) Multi-IO board.

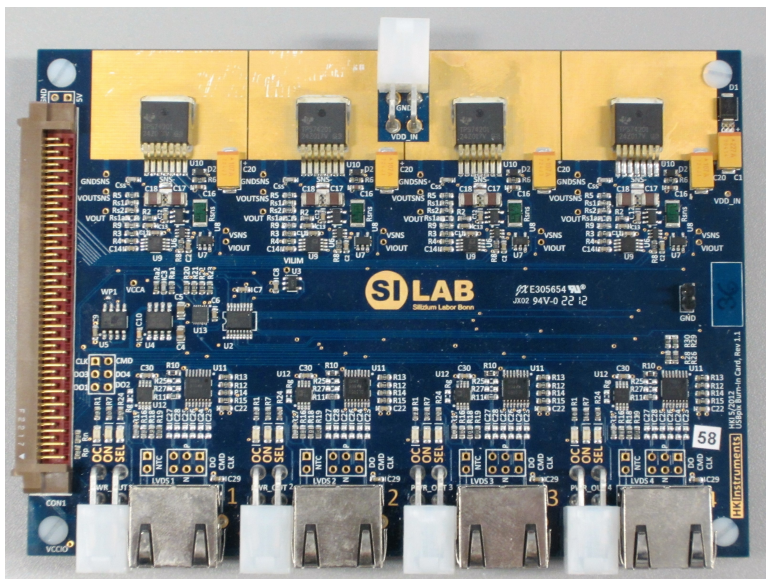

(c) Burn-In Adapter Card.

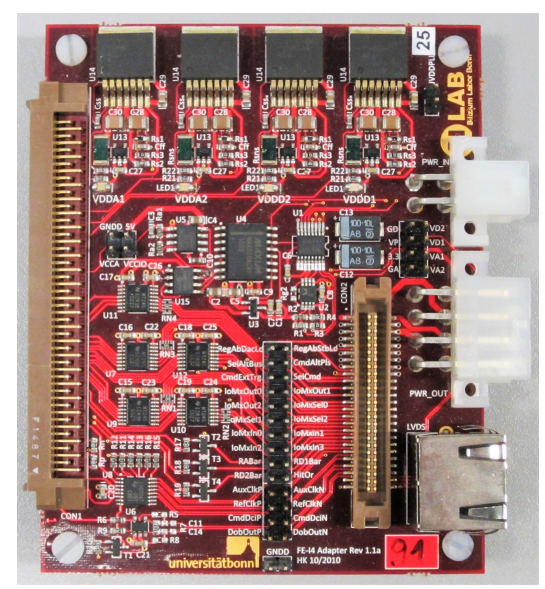

(b) FE-14 Adapter Card.

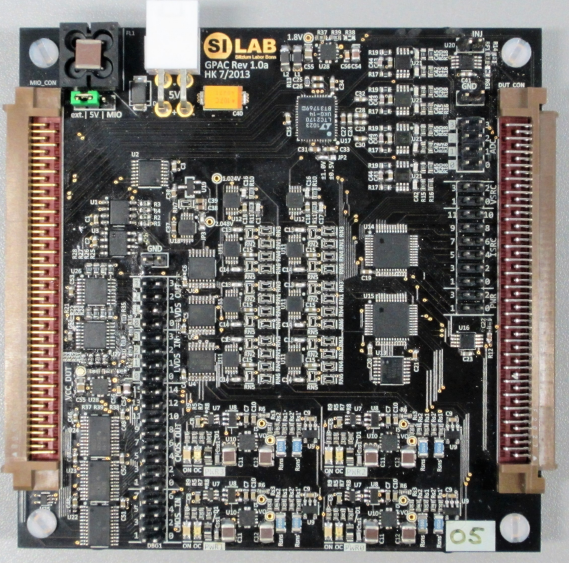

(d) General Purpose Adapter Card.

Figure 5.1: Photographs of the USBpix readout system Multi-IO board and the available adapter cards.

\subsubsection{STcontrol}

To access the hardware, the existing application software for the USBpix readout system, STcontrol is used. STcontrol is a collection of $\mathrm{C}++$ classes based on libraries used in the ATLAS Pixel readout software, providing an interface to configure and operate the connected assembly. The graphical user interface (GUI) is based on ROOT and Qt and the software package is platform-independent. A data analysis tool called Module Analysis completes the software package.

STcontrol enables the user to load, access and change the chip configurations in the GUI. In addition, various scan routines are available. Two different types of scans are implemented: calibration and source scans. In the latter, the charge is injected by an external source (e.g. radioactive source or laser), whereas in the first, the readout chip internal injection mechanism is used. During the scans it is possible to vary parameters like configuration, timing or power parameters in an adjustable range in different loops. At the end of every parameter loop, specific actions, e.g. parameter settings, can be 
executed. In addition, every loop can optionally be performed for certain masks or trigger conditions.

The resulting front-end data is received by the FPGA of the Multi-IO board, where it is synchronised, decoded and hit histograms are produced. These histograms are further analysed in STcontrol. Furthermore, it is possible to obtain the raw front-end data stream with trigger data inserted.

\subsubsection{Standard Scans}

As described in the previous section, various scan routines are implemented in STcontrol, which are used to characterise prototypes in a standardised way. A selection of these scans will be explained in the following, including the FE-I4 readout chip parameters (see Section 4.2.2), which are varied during the scans.

During the Analog and Digital Test the functionality of the analogue and the digital part of each pixel cell is tested. Via the internal charge injection circuit, a defined charge is injected multiple times directly into the discriminator (Digital Test) or the amplifier (Analog Test) of each pixel. Typically, the charge is chosen to be large enough to cause a hit. The number of hits that is read out is supposed to match the number of injections for a fully functioning module.

The charge is not injected into all pixels at the same time, but only into a selection of all pixels to not exceed the maximum readout chip occupancy. The preset masks for Digital and Analog Tests enable charge injection into every sixth pixel at the same time. The mask is shifted by one pixel in the next loop and the whole readout chip is scanned after six steps. These two scans test if the module is working properly and responds as expected to basic signals.

The discriminator threshold of the readout chip is determined by performing several Analog Tests with different injection charges. This scanning routine is called Threshold Scan and the injected charge is controlled by the PlsrDAC value. The scan result is the occupancy as a function of the injected charge. Ideally, the occupancy follows a step function with no hits below the threshold charge and above the threshold all injections cause a hit. But due to noise, the step function is smeared out. Figure 5.2 shows a possible result of a Threshold Scan. The data is fitted by a convolution of a step function and a Gaussian, describing the hit probability $p_{\text {hit }}$

$$
p_{\text {hit }}(Q)=\frac{1}{2} \operatorname{Erfc}\left(\frac{Q_{\text {thresh }}-Q}{\sqrt{2} \sigma_{\text {noise }}}\right),
$$

in which $Q$, the charge of the injected pulse, is given in DAC units, $Q_{\text {thresh }}$ is the charge that corresponds to the discriminator threshold, and $\sigma_{\text {noise }}$ is the equivalent noise charge (ENC) of the detector-amplifier system. Erfc is the complementary error function defined by

$$
\operatorname{Erfc}(x)=2 \int_{x}^{\infty} e^{-x^{\prime 2}} \mathrm{~d} x^{\prime} / \sqrt{\pi} .
$$




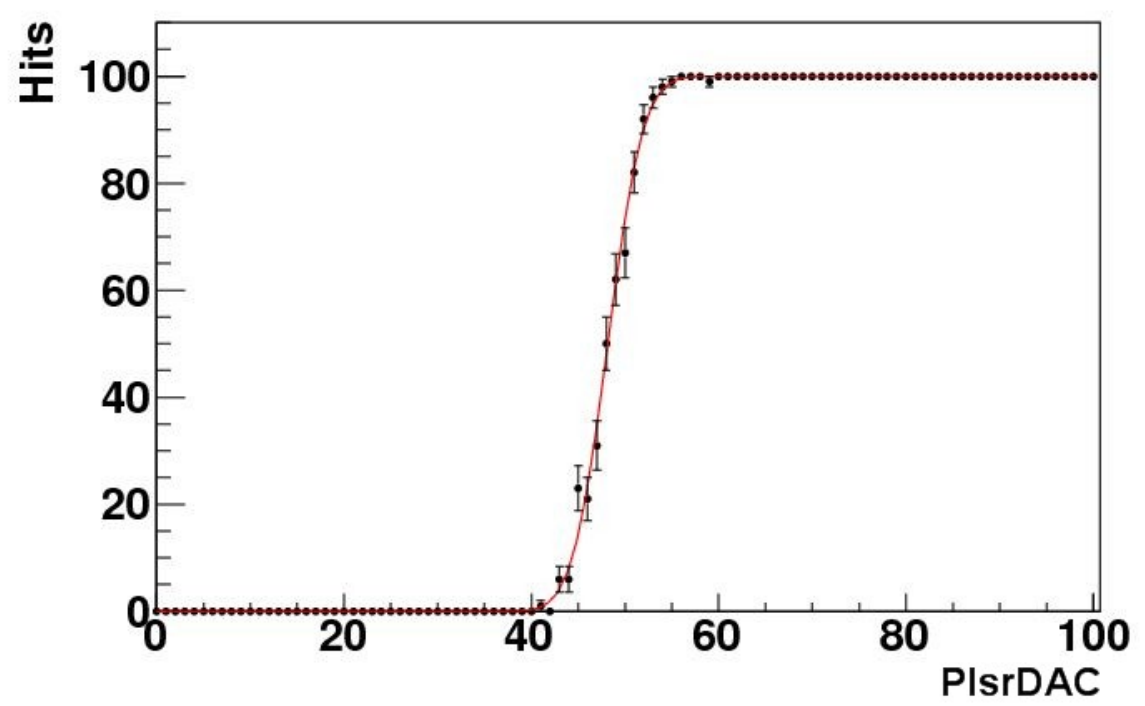

Figure 5.2: Result of a Threshold Scan. Number of hits versus the PIrsDAC value, parametrising the injected charge and fitted with Equation 5.1.

This function is referred to as S-curve.

To convert the threshold charge value from DAC units into a real charge, the charge injection mechanism is calibrated. Calibration scans are implemented in STcontrol to determine the conversion factor $V_{\text {Grad }}$ and the calibration offset $V_{\text {offset }}$. The injected charge $Q_{\text {inj }}$ is

$$
Q_{\text {inj }}=C_{\text {inj }}\left(P l s r D A C \cdot V_{\text {Grad }}+V_{\text {offset }}\right),
$$

with the chosen injection capacitance $C_{\text {inj }}$, which vary over the chip due to production. The values $C_{\text {inj }}$ of the capacitors, which are entered into the configuration are taken from simulations of the chip layout leading to a large uncertainty on the injected charge.

The global threshold value is adjusted with a global DAC (GDAC) tuning procedure. The parameters Vthin_Alt_Coarse and Vthin_Alt_Fine influence the global threshold setting. A charge corresponding to the target threshold is injected and the hit occupancy is monitored during the scan. If the percentage of injections that result in a hit is less than $50 \%$ the parameters are decreased by a certain step size and if it is more than $50 \%$ they are increased. After a fixed number of iterations with decreasing step sizes, the final Vthin_Alt_Coarse and Vthin_Alt_Fine values are entered into the FE configuration. To compensate for differences among the pixels, an in-pixel adjustment is done by a trim DAC (TDAC) tuning procedure, which works similarly to the adjustment of the global parameter.

Like the threshold, the feedback current is adjusted globally by the PrmpVbpf parameter and with the FDAC per pixel. The purpose of the tuning of the feedback current is an adjustment of the $T o T$ response to a certain charge, for example $T o T=7$ at a charge 
of 14000e. For in-pixel adjustment, the FDAC is scanned and the ToT response to the fixed charge injection is measured. The best matching value for each pixel is chosen and entered into the FE configuration.

\subsection{CMOS Sensor Integration into STcontrol}

In contrast to a passive sensor, the active CMOS sensor needs a configuration and several bias voltages. Thus, during this thesis, the USBpix readout system and its software were expanded to provide the required CMOS functionalities. The General Purpose Adapter Card (GPAC) provides the additional bias voltages and an external injection mechanism. Consequently, it is possible to operate the CMOS module without further external power supplies. All the FE-I4 readout chip functionalities were ported to the usage with the GPAC. In addition, the CMOS sensor specific functionalities, like the handling of the configuration, were implemented in STcontrol. With this extension, it is possible to perform scans with simultaneous setting of CMOS sensor and readout chip parameters.

Figure 5.3 shows the set-up used for the operation with CMOS sensor-readout chip modules, the USBpix Multi-IO board (MIO), the GPAC and the CCPD printed circuit board (PCB), which carries the prototype module. In the following, the implementation of the

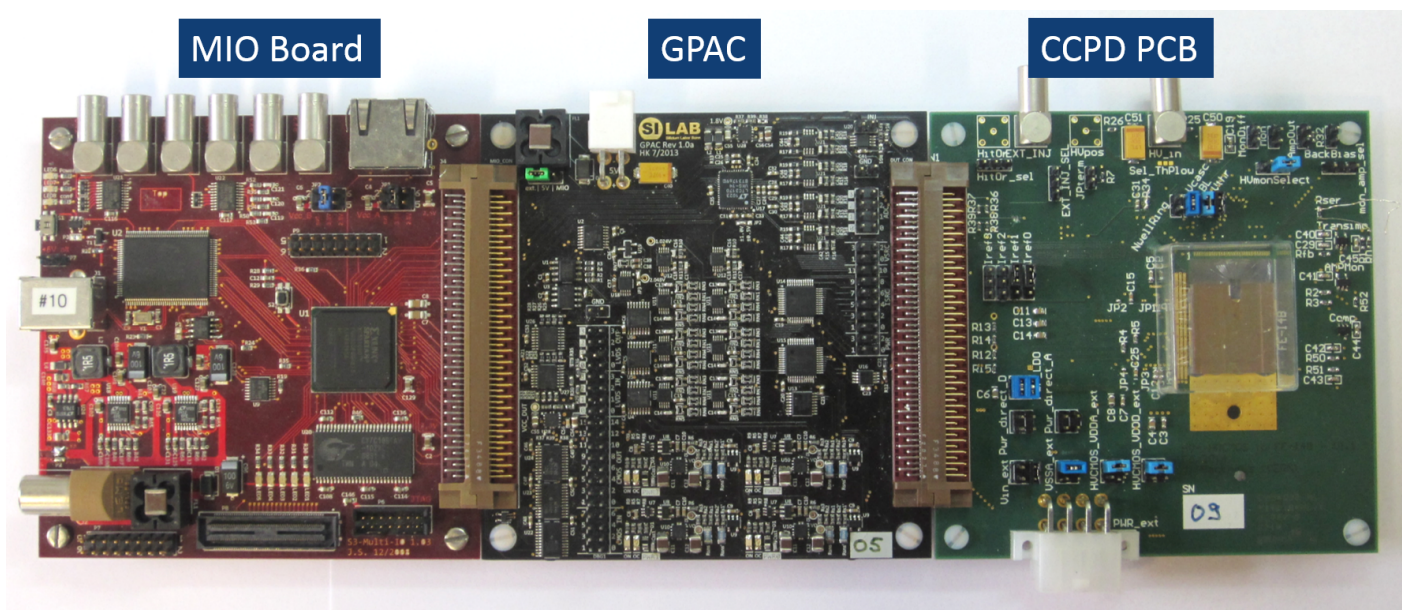

Figure 5.3: Picture of a USBpix readout system with Multi-IO (MIO) board, General Purpose Adapter Card (GPAC) und CCPD PCB.

CMOS sensor specific functionalities in STcontrol and the general structure is described according to [57]. STcontrol is a modular software with three main components: PixModule, PixController and USBpixdll (see Figure 5.4), which will be described in the following.

\subsubsection{PixModule}

PixModule contains object classes, which represent the hardware components, e.g. PixFE for the different versions of readout chips or PixMCC for the Module Control Chip chips. These classes are abstract base classes and provide generic functionalities, e.g. read and 


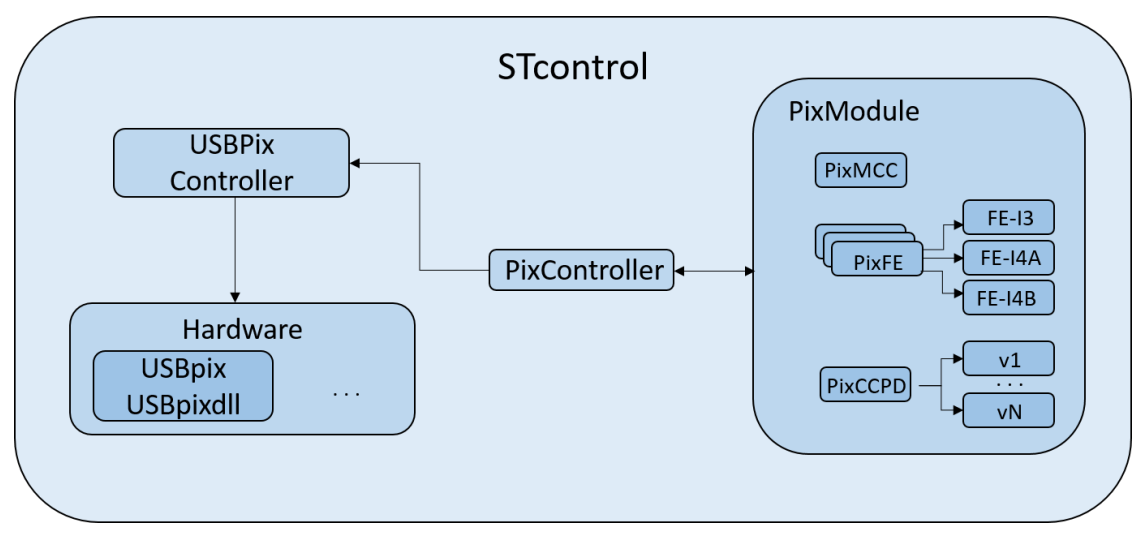

Figure 5.4: Schematic drawing of the modular structure of STcontrol.

write commands. The PixCCPD base class was added to manage the requirements for the active sensor flavours. The implementation of the individual versions and flavours is contained in the derived classes, e.g. PixFE-I4A and PixCCPDv2.

The various configuration parameters are listed and accessed by the higher level classes and the GUI via plain-text labels, allowing dynamic access to the configuration items of different chip versions. The version specific classes contain the actual register configuration. As there are different sensor designs in the CPIX demonstrator programme, this implementation was chosen to easily add support for new sensor layouts. When a CCPD version is added to PixCCPD, it inherits from the abstract class. Thus, the translation into the GUI is done automatically and it is possible to use all configuration parameters as scan variables.

\subsubsection{PixController}

The communication between software and hardware is taken care of by the PixController. This class is another polymorphic class, which handles different hardware interfaces, like USBpix, ATLAS readout hardware or other test systems. The scan processing and the main scan settings are handled here. The advantage is that any PixCCPD register as well as parameters of connected power supplies or any GPAC parameters are available as scan parameter.

The structure allows a seamless integration of further features in addition to the already implemented readout chip operations without changing them and using them together with the new features. The PixController structure enables to inherit much existing functionality for the CCPD because of the dynamic configuration access.

\subsubsection{USBpixdII}

The third component is the interface library USBpixdll, which is the hardware interface. It handles the communication between the controller and the hardware by generating and sending bit streams, synchronous command management and trigger control. To support the CCPD, two main components were added. On the one hand the handling of the sensor specific bit streams and on the other hand the handling of the GPAC injection. 
The USBpixdll has one main control interface, which receives commands from the PixController and executes these commands. Furthermore, the USBpixdll generates the bit streams, which are hardware specific. Therefore, the hardware specific interface classes, e.g ConfigFEMemory and ConfigCCPDMemory, contain the information about the different command and register structures. During the integration of the CCPD support, this specific class for the CCPD was added. This class receives the parameter values coming via the PixController from the GUI. It distinguishes between the various sensor flavours and constructs the dedicated register structure. The pixel information from the PixController is translated to the correct bit sequence and is stored in a bit array.

The actual bit array data is sent to the CCPD along with generating the corresponding clock depending on bit stream type (pixel or global). This sequence is followed by the load signal.

The GPAC injection is time-critical and thus it was implemented in the FPGA. The injection period, duration and the number of injections is submitted by the USBpixdll to the FPGA.

\subsubsection{Newly Implemented Scans}

With the integration of the CMOS sensor in the USBpix readout system, it is possible to simultaneously access the configuration parameters of the readout chip and the active sensor during one scan. Using this functionality, it is possible to tune the readout chip response to the signal of the active sensor.

To test the active sensor a so-called Analog Test CCPD was implemented, which is similar to the Analog Test of the readout chip. A well-defined charge is injected directly into the analogue part of all pixels of the active sensor at the same time via the GPAC injection circuit. The generated signal is then read out by the readout chip. With this Analog Test $C C P D$ the sensor, the capacitive coupling and the readout chip can be tested.

In addition, a new so-called Threshold Scan CCPD is available to obtain the threshold voltage and the equivalent noise voltage of the active sensor. The injection voltage provided by the GPAC is controlled automatically during the scan and thus the injected charge is varied. Similar to the normal Threshold Scan (see Section 5.1.2), the number of hits per injection voltage is measured and fitted with an S-curve.

A first tuning of the readout chip parameters to a signal from the active sensor is the new FDAC Tune CCPD. By tuning the FDAC of the readout chip, the ToT response is adjusted to a certain signal from the active sensor. This procedure is important for a successful subpixel mapping.

\subsection{Test Beam Set-Up}

To study the prototype module performance under realistic conditions, test beam measurements are one of the most important techniques. A particle beam is provided by an accelerator and a test beam telescope, which consists of several well known pixel sensors, is used to reconstruct the particle tracks. Consequently, the point of impact on the prototype module is interpolated. Using this information, key quantities like the hit efficiency are determined for the device under test (DUT).

The test beam measurements for this thesis were performed at the German Electron 
Synchrotron (DESY) and the EUDET telescope [58] was used. The track reconstruction was done with the EUTelescope software and the final data analysis with the TBmonII framework. All these components are described in the following.

\subsubsection{Test Beam Facility}

The German research centre for accelerators, photon science and particle physics, DESY is located in Hamburg and was founded in 1959. Important accelerators are based at this side and many interesting experiments in particle physics took place at DESY.

The starting point of the beam for test beam measurements is the DESY II accelerator [59], which collides electrons or positrons. The particle beam is guided to a primary target (see 5.5), which is a carbon fibre, and Bremsstrahlung is generated. On a secondary target, a

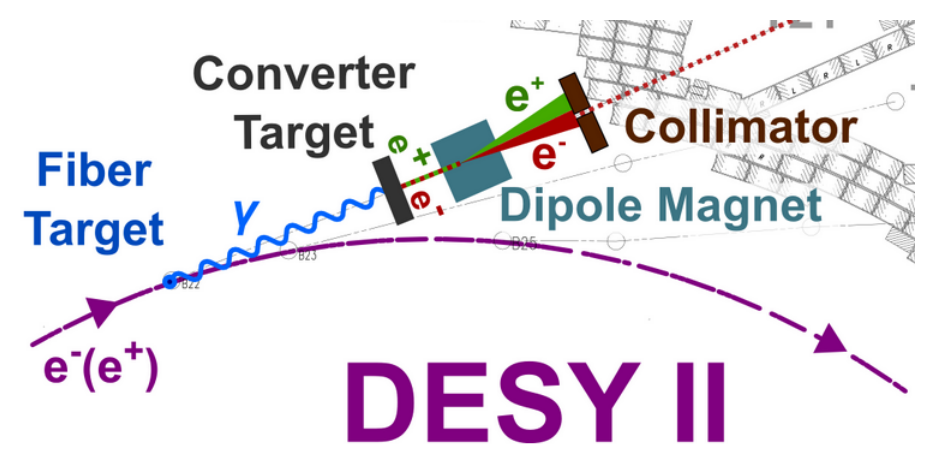

Figure 5.5: Schematic layout of the beam production for test beam measurements.

metal plate, the photons are converted to electrons and positrons, which are separated in energy and charge by a dipole magnet. The energy of the particles used for the test beam measurements can be varied between $1 \mathrm{GeV}$ and $6 \mathrm{GeV}$ by changing the dipole current. Finally, the continuous beam is shaped by a collimator.

\subsubsection{Telescope Sensors}

The EUDET telescope is equipped with six MIMOSA26 [60] sensors. Figure 5.6 shows a photograph of the telescope, which was taken at one of the CCPD test beam measurements. The telescope has six sensor planes on two arms and two DUTs in the middle. The telescope needs to have a very good track resolution to enable subpixel sensor studies. The MIMOSA26 sensors have an intrinsic spatial resolution of $5.3 \mu \mathrm{m}$, determined by the pixel size of $18.4 \times 18.4 \mu \mathrm{m}^{2}$. This resolution is improved by the use of cluster information to below $4 \mu \mathrm{m}$.

A low material budget of the sensors is required to reduce the effect of multiple scattering and achieve a good telescope performance. Monolithic active pixel sensors, like the MIMOSA26, combine the sensitive volume and the readout circuitry on the same substrate. Thus, the thickness is only a few tens of micrometers, including a $14 \mu \mathrm{m}$ thick epitaxial layer and the readout electronics. Every pixel has an amplifier and a correlated double sampling circuit to reduce the noise. It is read out in a column-parallel mode and each column is equipped with a discriminator for analogue to digital conversion. The 


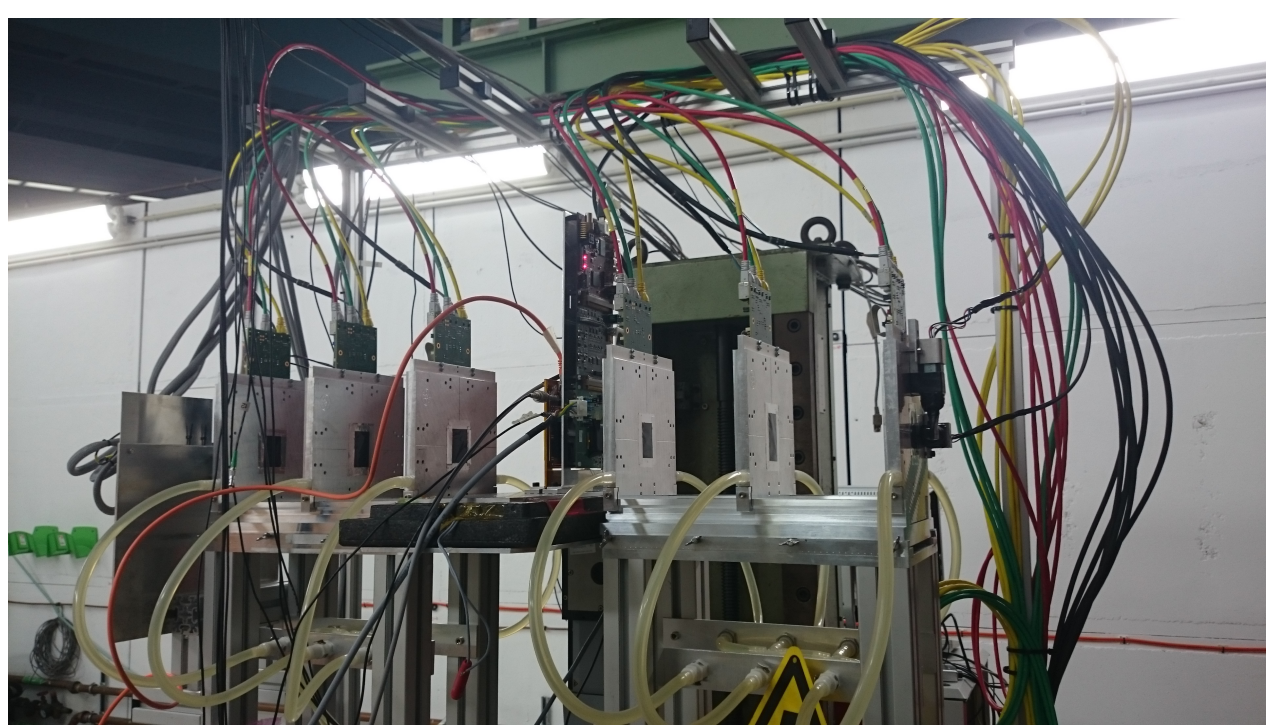

Figure 5.6: Photograph of a EUDET telescope with six MIMOSA26 sensors and two DUTs in the middle. On the right hand side two scintillators for triggering are visible.

integration time is about $112 \mu \mathrm{s}$, which is long compared to the $25 \mathrm{~ns}$ of the ATLAS Pixel Detector modules. Thus, a reference plane is used to improve the timing. The data rate is reduced by zero-suppression and binary readout.

To test prototype modules of different sizes, the sensitive area of the telescope is supposed to be as large as possible. The MIMOSA26 sensors have an active area of $2 \times 1 \mathrm{~cm}^{2}$, which is a reasonable size. A $0.35 \mu \mathrm{m}$ CMOS process is used to produce the sensors, which have a pixel matrix of 1152 columns and 576 rows.

\subsubsection{Triggering and Data Synchronisation}

The control of the triggering and the synchronisation of the data streams is managed by the Trigger Logic Unit (TLU) [61]. It is an FPGA board that receives signals from a triggering device, like four scintillators in front and behind the telescope or a region-ofinterest plane. When a particle passes through the telescope, the triggering device sends a signal to the TLU. The TLU generates a trigger signal and distributes it to the readout system of the telescope and the DUT. The TLU inputs can be checked for a coincidence between the signals. If the DUT is smaller than the scintillator area, the region-of-interest plane is used to decrease the triggering area and consequently, to decrease the recorded events without hit in the DUT. An FE-I4 module with a planar sensor that has only a small part of its pixels enabled is an example for a region-of-interest plane.

To synchronise the data streams of the different readout systems, a consecutive trigger number is added by the TLU to all data streams. In addition, the TLU can receive a signal from the readout systems, if a device is busy, e.g. because it is being read out. As long as the busy signal is present, no more triggers are sent to the devices. 


\subsubsection{Data Acquisition Software}

All components involved in test beam measurements are controlled by the EUDET data acquisition system (EUDAQ) [62]. It communicates with all hardware components via TCP/IP connection. The main program Run Control is a graphical user interface, to control the data taking and display the hardware statuses. It allows the user to initiate configurations and start and stop the data acquisition. All hardware components, e. g. the telescope and the DUTs have their own program connected to Run Control, which is referred to as a Producer (see Figure 5.7). To configure and interface the TLU, the TLU

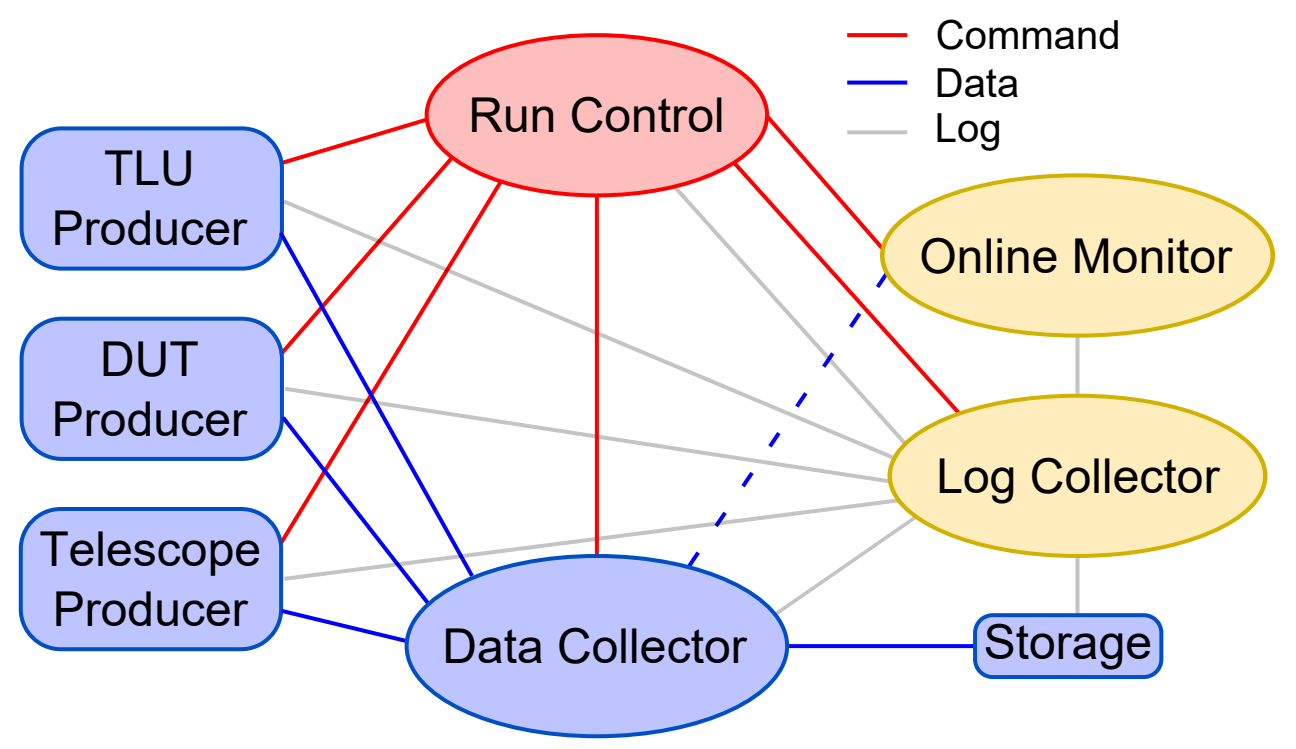

Figure 5.7: Schematic drawing of the components of the EUDAQ system. The red lines represent command actions, the blue lines data transfer and the grey lines log information. The drawing is adapted from [63].

Producer is used, which sends the time-stamps to the Data Collector. All data streams are collected from the devices by the Data Collector, merging them into one file, which is written to the hard disc. Additional programs monitor log messages and provide online plots of the different planes. The configuration of all components is done via one central configuration file.

\subsubsection{Track Reconstruction}

The track reconstruction is done in the EUTelescope software framework, which calculates the tracks passing the telescope from the raw telescope data. It processes the data in five steps: data conversion, clustering, hit making, alignment and track fitting. The basic principle of these steps are described in the following. For a detailed description see Reference [64].

In the first step, the different raw data from the readout systems are converted into a uniform format. In addition, noisy pixels are identified and marked for the further steps. In the clustering step, for every plane, hit pixels that are caused by the same incident 
particle are grouped. The global hit positions of the identified clusters are obtained in the hit making step. Furthermore, a prealignment is based on the correlations between hits on neighbouring planes as a starting point for the fine alignment. The alignment is necessary, because the positions of the sensors have to be known with a better precision than the pixel size, which cannot be determined with a mechanical measurement alone. The MillepedeII [65] tool is used for the alignment and a preliminary track fit is done. The final step of the track reconstruction is the track fitting. All results from the previous steps are used and the Deterministic Annealing Filter [66] concept is used for the track fitting.

The output of the track reconstruction process is a file that contains all raw data of the DUTs as well as the reconstructed hit positions from the fitted tracks.

\subsubsection{Analysis Framework}

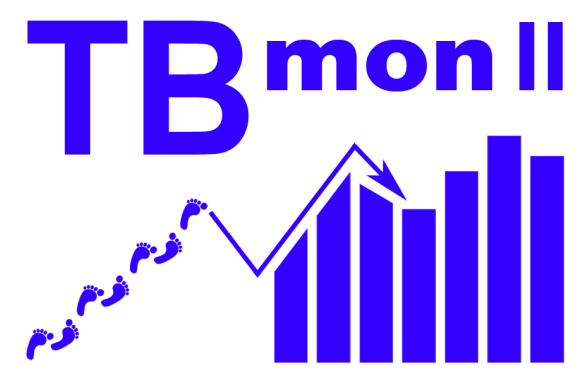

Figure 5.8: TBmonll Logo.

To further analyse the output of the track reconstruction, TBmonII is used, which is a standalone $\mathrm{C}++$ based analysis framework. It is a successive development of TBmon, which was the standard tool for the analysis of IBL test beam campaigns. TBmonII has a more user friendly structure, while most analyses were ported from the previous version. One of the most important changes is that general parameter settings, DUT geometry and analysis settings are read in from configuration files and are not hard-coded as in the previous version.

In addition, TBmonII offers the possibility to enter special DUT geometries for every DUT individually. Each DUT configuration can contain an unlimited number of different pixel types, which can be repeated with a given periodicity and can have different initial coordinates. For further information on the TBmonII implementation of arbitrary pixel geometries see Reference [67]. This possibility of the usage of multiple pixel geometries is necessary for studies of the subpixel structure of the HV2FEI4.

Before an analysis is performed, the raw track data input is preprocessed. Noisy and their neighbouring pixels are masked, as well as dead pixels. In a second step, the raw hits are grouped into clusters that are caused by one incident particle. Furthermore, the alignment of the track reconstruction is reviewed. In the process, the offset between the residuals and the hit positions is calculated and consequently the tracks are shifted by this offset value. 
Finally, several selection criteria are assigned to the tracks. One important criterion is that the track is matched. This term means that the interpolated track position is associated with a hit in a pixel within a certain matching radius to this position. In addition to this requirement, a matched track in the reference plane is requested. The matching radius for the reference plane can be selected independently. The additional hit in the reference plane is necessary, to consider only tracks that are within the active time window of the FE-I4 readout chip. After a trigger signal the active time spans of the DUTs and telescope planes are different. The readout time of the telescope planes is about $112 \mu \mathrm{s}$, whereas the FE-I4 is only active for $16 \times 25 \mathrm{~ns}$ after a trigger signal. With the criterion of a matched track in the reference plane only tracks within the active time of the FE-I4 are considered for further analyses.

Further requirements on the tracks are set, which are described in the following list.

- A matched track in the reference plane is required to only consider tracks within the active time span of the FE-I4 readout chip.

- The $\chi^{2}$ of the track fit is supposed to be below a certain value to assure a good track fit quality. The default value is 25 .

- The track is not allowed to pass an edge pixel, which are considered in a special analysis, but it needs to be in the central region.

- The track does not hit a masked pixel to not influence the total cluster charge.

A track is defined as good, if all these selection criteria are passed.

The raw hit information of the DUT, which includes the hit position (in column and row), the time-stamp and the ToT, and the positions of the fitted tracks are evaluated in several analyses. Most analyses offer results with different selection criteria applied, e.g. before and after matching. One of the most important tasks is the calculation of the DUT hit efficiency, which is given by the number of good tracks with a matched DUT hit divided by the total number of good tracks. The hit efficiency is calculated per pixel and as in-pixel efficiency. For the latter, all pixels with the same geometry are mapped to one pixel to increase the statistics under the assumption that pixels of the same geometry behave in the same way. Furthermore, analyses about the timing, the cluster size and the ToT are available, as well as the residuals, which provide information about the telescope pointing resolution and the single pixel resolution. All analyses are explained in [64] and [67]. 


\section{HV2FEI4 Characterisation}

The characterisation of a hybrid module consisting of an HV2FEI4v2 sensor and an FE-I4B readout chip is described in this chapter. Some basic properties of the sensor can be found in Section 4.4 and information on the readout chip in Section 4.2.2.

The pixel matrix of the HV2FEI4v2 is based on unit cells that contain six sensor pixels. The entire pixel matrix is produced by copying twelve unit cells in $x$-direction and 20 unit cells in $y$-direction. Figure 6.1 (left) shows the described structure. It should be noted that every second cell is mirrored on the $y$-axis. The 24 HV2FEI4 rows are counted in negative $x$-direction (long pixel dimension) and the 60 columns in positive $y$-direction. The investigated version of the HV2FEI4 contains three types of pixels (see Figure 6.1 right top). Type A is designed to be radiation hard by using circular feedback transistors. Their gain is supposed to be smaller due to a higher feedback capacitance. Most of the pixels (36 columns / 12 unit cells) are type A2, which is called partially radiation hard.
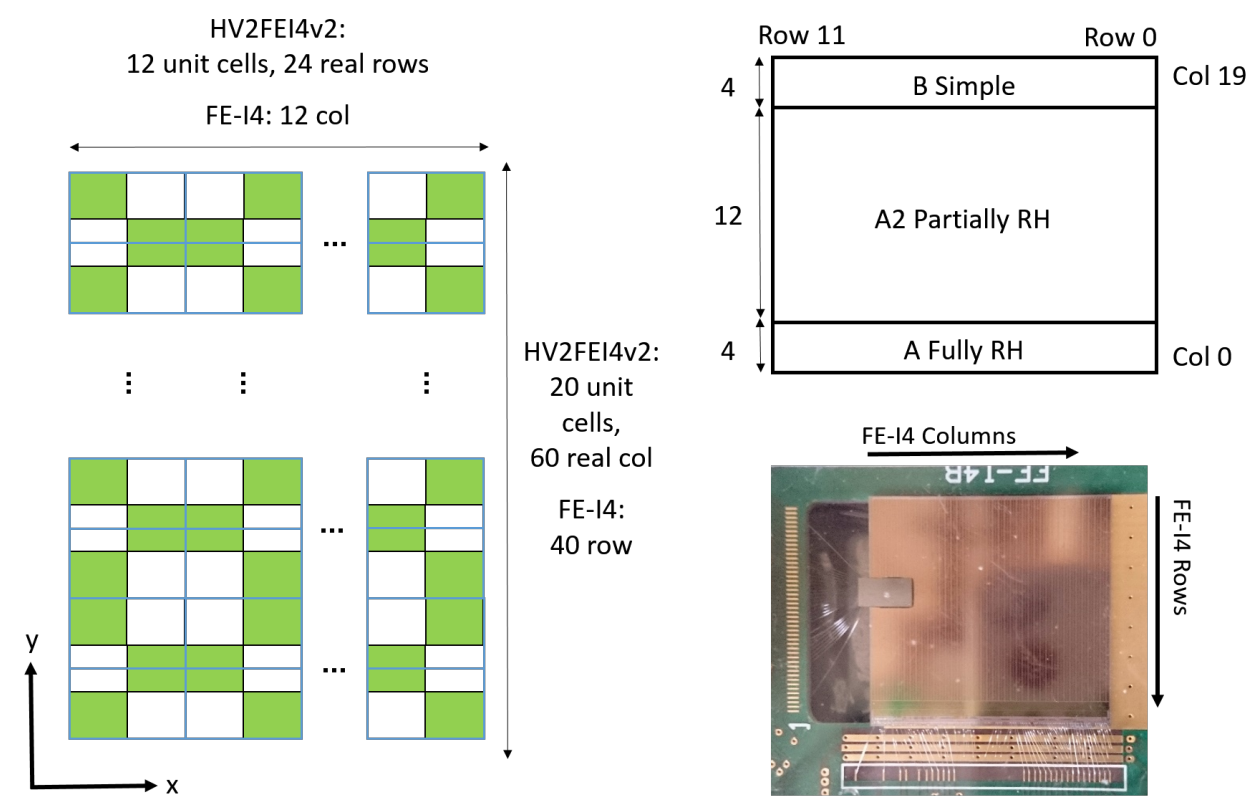

Figure 6.1: Schematic drawing of the HV2FEI4V2 sensor layout and the orientation in the FE-14 readout chip coordinate system. The left drawing shows the 20 unit cells in $x$-direction and 12 unit cells in $y$-direction and the pixel orientations. The right-hand top drawing shows the different pixel types that are contained in the HV2FEI4V2. The right-hand bottom picture shows the sensor glued to the FE-I4B, whose column and row orientation is marked. 
Type B pixels, which are called simple pixels, contain simplified electronics. Simulation showed a smaller detector capacitance and a higher bias current. They need a different configuration sequence and cannot be configured together with the other pixel types. To simplify the testing procedure, they are disabled in all further measurements.

The six pixels of a unit cell are connected to two FE-I4 readout chip pixels. The schematic drawing in Figure 6.2 shows the subpixel mapping for one unit cell. The output signals

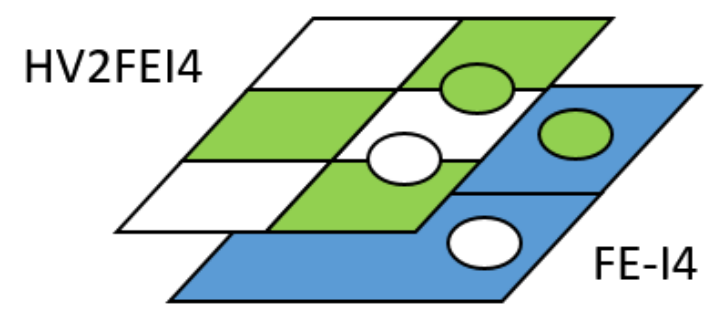

Figure 6.2: Schematic drawing of the HV2FEI4 subpixel structure of one unit cell. The three white (light) sensor pixels are connected to the FE-14 readout chip pixel with the white pad and the green (dark) ones to the FE-14 pixel with the green pad.

of the three green and the three white pixels are summed and connected to the corresponding green or white readout pixel contacts. The signal amplitude of each subpixel can be adjusted via the parameter VNOut $1 / 2 / 3$ for subpixel $1 / 2 / 3$. The FE-I4 readout chip measures the amplitude of the subpixels output signals as ToT. This measurement allows the assignment of a hit to a specific subpixel.

\subsection{Configuration}

The schematics of the electronics of one standard pixel cell are presented in Figure 6.3. The components form a readout cell consisting of an amplifier, the corresponding feedback circuit and a comparator.

The working points of the transistors are set by external bias voltages. These voltages are generated outside the chip on the GPAC and connected through wire bonds directly to the sensor. The most important voltages are the external threshold voltage, $T h$, and the baseline voltage, $B L$. An overview over the standard voltages is given in Table 6.1. Other bias voltages are generated in the bias block, which is placed in the chip periphery. The generation is controlled by the sensor configuration. As described in Section 5.2, the writing of the sensor configuration was implemented in STcontrol. Using shift registers, the global and pixel DACs are written, which control the bias block.

\begin{tabular}{l|c|c|c|c|c} 
Parameter & BL & Th & VGate & Vcasc & ThPlow \\
\hline Standard Value [V] & 0.8 & 0.89 & 2.1 & 1.2 & 0.8
\end{tabular}

Table 6.1: Standard values for the external bias voltages of the HV2FEI4v2 sensor. 


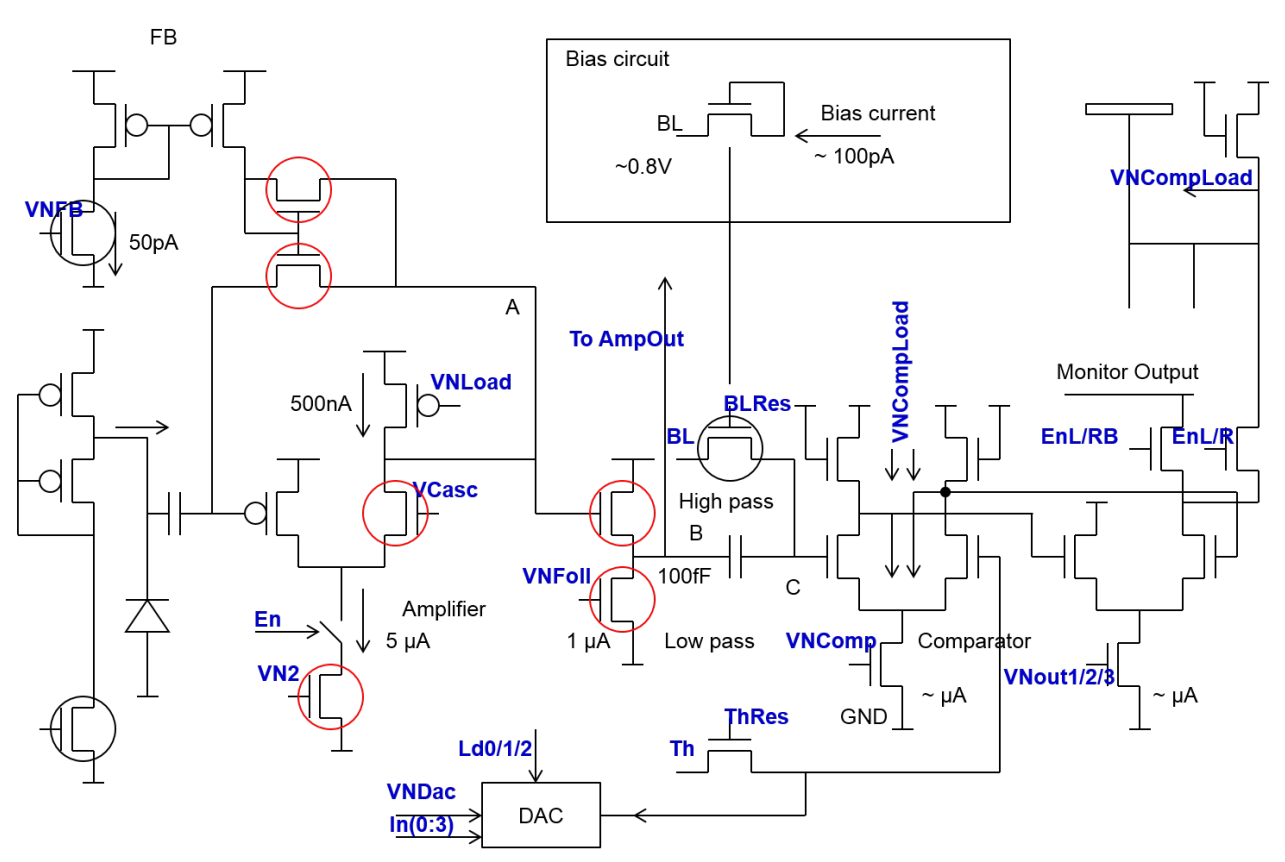

Figure 6.3: Schematic drawing of the pixel electronics of a HV2FEI4v2 type A2 cell [68].

The global register consists of 19 6-bit DACs and six switches. The adjustment of the feedback current is controlled by the VNFB DAC. The threshold step size is influenced by the ThRes DAC. Furthermore, the parameters VNOut1, VNOut2 and VNOut3, which

\begin{tabular}{l|c|c|c|c|c} 
Parameter & VNFB & ThRes & VNOut1 & VNOut2 & VNOut3 \\
\hline Standard Value [DAC] & 1 & 20 & 60 & 60 & 60
\end{tabular}

Table 6.2: Standard values for the global DACs of the HV2FEI4v2 sensor.

were already mentioned, adjust the amplitude of the output signal of the three different subpixels. The standard global DAC settinges are presented in Table 6.2.

Figure 6.4 shows the sequence of the total pixel shift register, which is separated into various parts for the unit cell rows and columns. Every unit cell row sequence (row 0 to row 11) consists of 16 bits (see Figure 6.5). The 4-bit InR/L DACs adjust the comparator threshold for the pixel row and are comparable to the TDAC for the FE-I4. The EnR/L DACs connect the comparator output signal to the monitor output of the sensor or to the FE-I4 readout chip. Furthermore, the En DACs enable or disable certain pixels in the whole row.

SRin $\longrightarrow$\begin{tabular}{|l|l|l|l|l|l|}
\hline row0 & row1 \\
\hline
\end{tabular}$.$\begin{tabular}{|l|l|l|l|}
\hline row10 & row11 & col0 & col1 \\
\hline
\end{tabular}

Figure 6.4: Schematic drawing of the sequence of the pixel shift register. 


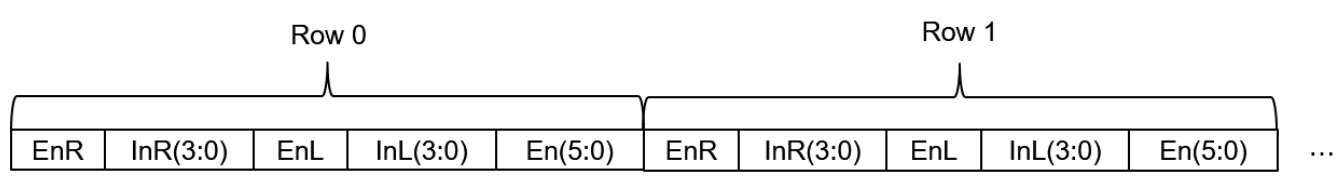

Figure 6.5: Schematic drawing of the sequence of the pixel shift register for two unit cell rows.

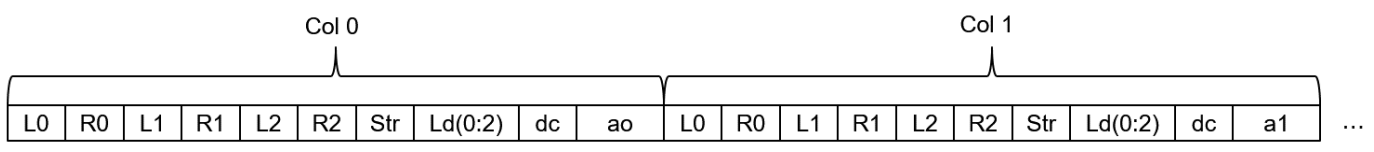

Figure 6.6: Schematic drawing of the sequence of the pixel shift register for two unit cell columns.

Figure 6.6 shows the column shift register sequence for two unit cells. The $L 1 / 2 / 3$ and $R 1 / 2 / 3$ DACs determine, which pixel output is connected to the monitor output together with the EnR/L DACs. Furthermore, the Str DAC handles the possible strip readout mode and the $d c$ DAC the direct coupling option. Both parameters are not used in the following. The $a_{i}$ DAC connects the amplifier output of the i-th unit cell column to an output pad. The same parameter is available for every unit cell column. This special output is only possible for one column at the time.

Finally, the $L d$ DACs control, which column the InR and InL DACs are written to. To assign a different InDAC value to every pixel, the whole shift register needs to be written 60 times. For example, if the unit cell in row 0 and col 0 is supposed to be written, InR and $I n L$ in row 0 are set to the desired value. Only the $L d$ DAC in column 0 is allowed to be non-zero, otherwise the unit cells in all columns in row 0 would get these $I n R$ and $I n L$ values.

At this point, two pixels of the unit cell in row 0 and column 0 are set to their InDAC value. The procedure needs to be repeated twice to set all six pixels of one unit cell to the desired value. To set all unit cells in the other columns to the specific InDAC values, the procedure needs to be repeated 20 times in total.

This configuration sequence was developed to allow to set different $I n D A C$ values for all pixels of the HV2FEI4v2 for the first time. In Figure 6.7a an oscilloscope screenshot of the datastream being sent to the shift register can be seen. The HV2FEI4v2 has four wire bond pads for the configuration inputs signals: global clock, pixel clock, data in and load. The different clock signals determine, which of the shift registers data is sent to. The load signal loads the shift register content into memory cells. Figure $6.7 \mathrm{~b}$ shows the complete sequence of 60 shift register write accesses. 


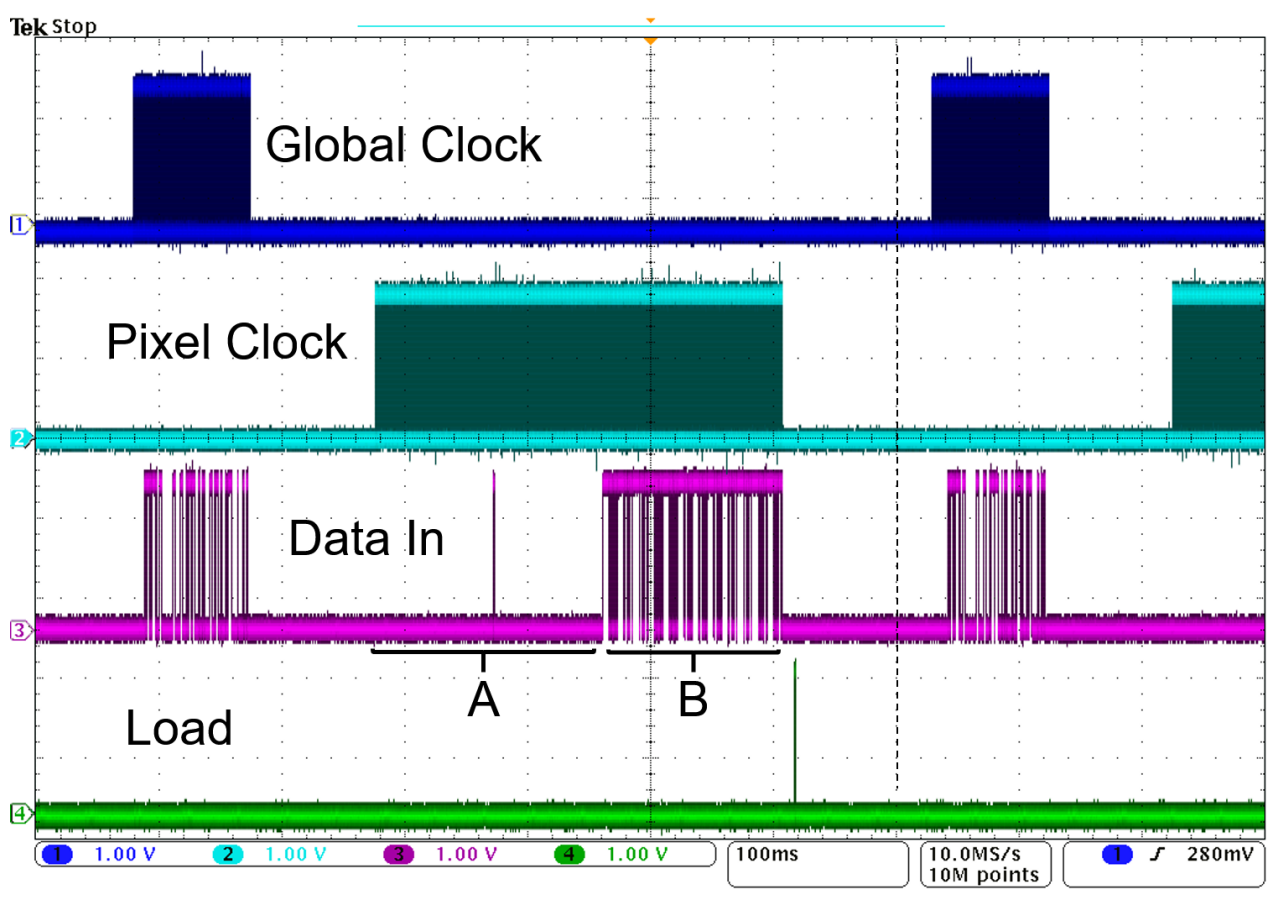

(a) Writing of one global and one pixel register.

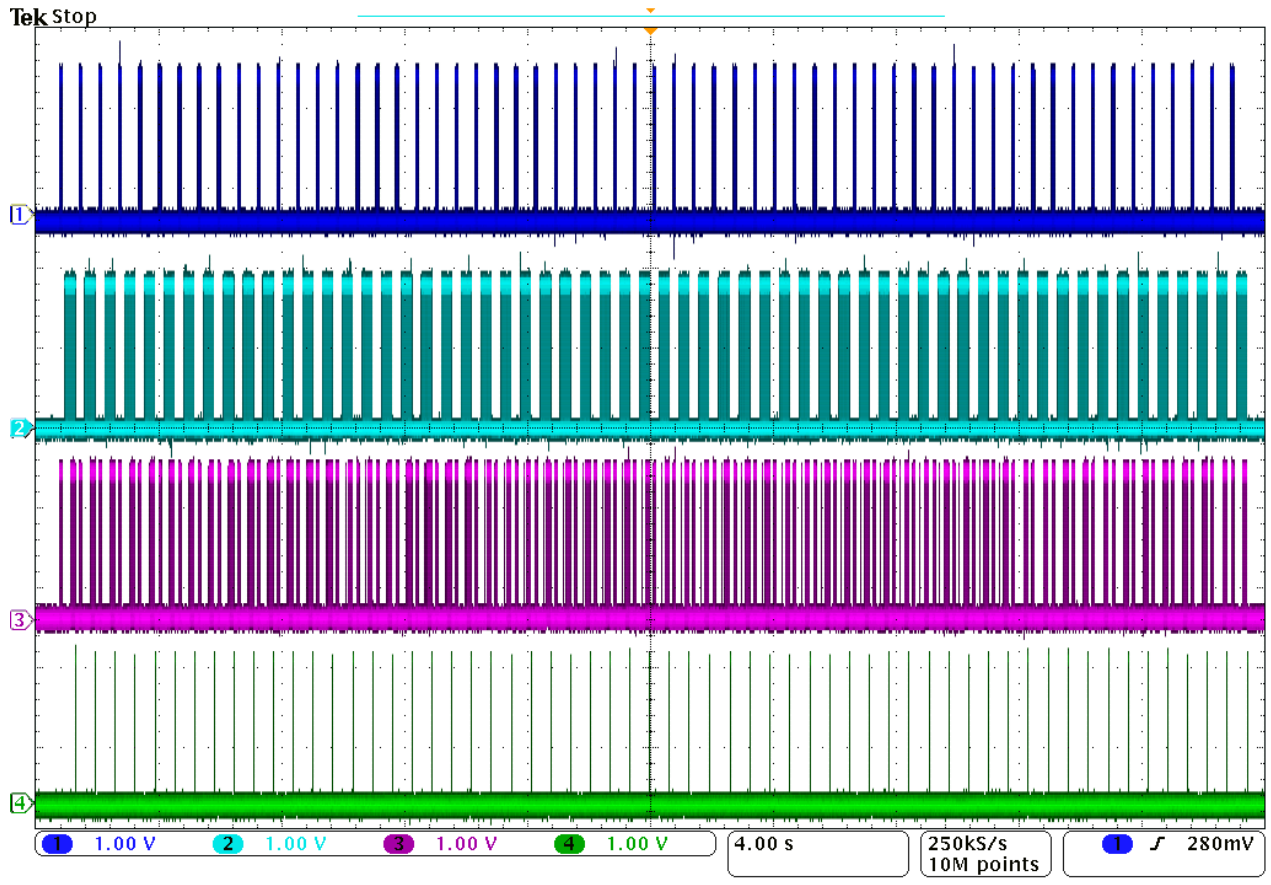

(b) 60 shift register write accesses to configure the whole pixel matrix.

Figure 6.7: Oscilloscope picture of the shift register write procedure. The global clock signal is shown in blue (top), the pixel clock signal in cyan (second line from the top), the data in signal in pink (third line) and the load signal in green (bottom). The data sequence for the 20 columns with one $L d$ signal is marked with $A$ and the sequence for the 12 row with $B$. 


\subsection{Parameter Studies}

\subsubsection{HV2FEl4 Amplifier Characterisation}

To characterise the HV2FEI4v2 sensor and confirm the correct writing of the configuration, the amplifier and the comparator output signals were investigated. Only one sensor column can be connected to the amplifier output pad at the same time. The number of pixels that are connected to the monitor output can be chosen by the configuration.

In Figure 6.8 the amplifier (blue) and comparator (cyan) response is displayed after a charge injection by the GPAC (green) into all pixels. The total comparator output signal

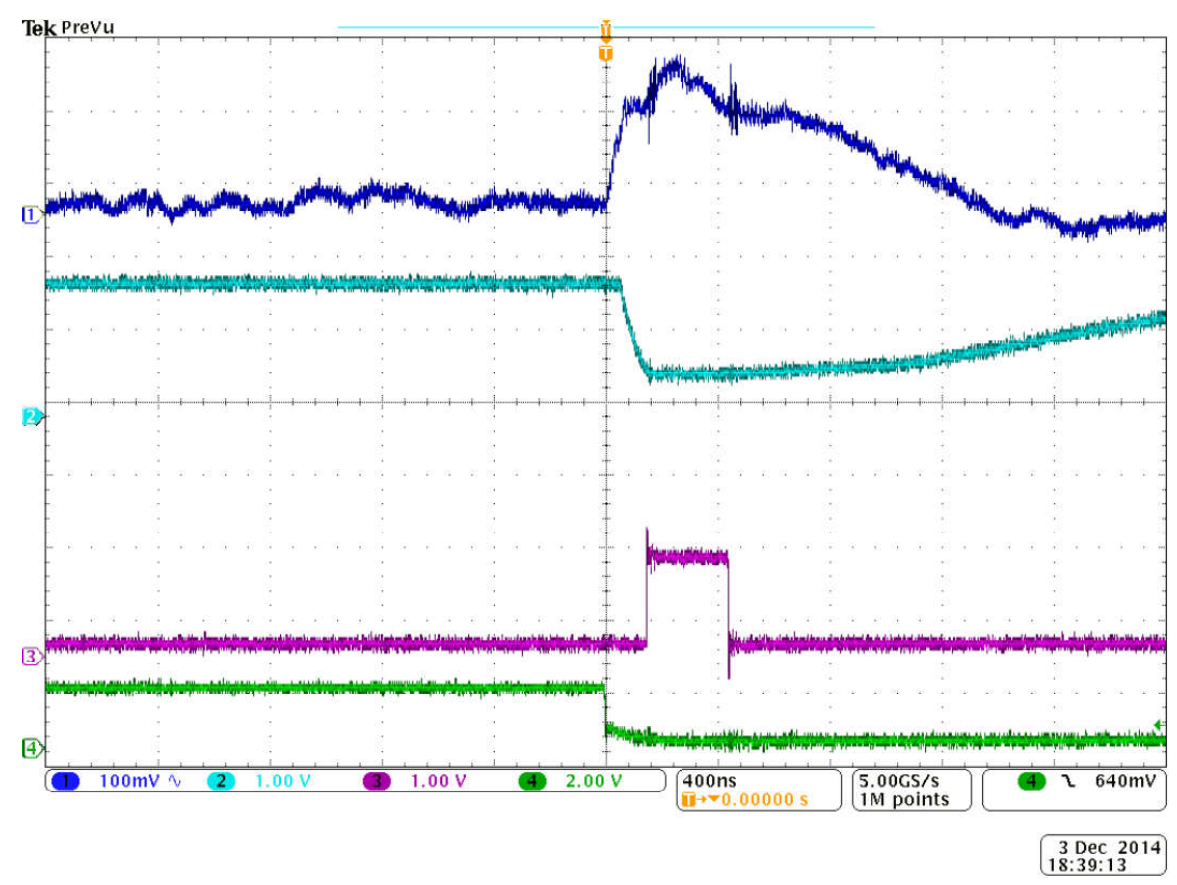

Figure 6.8: Oscilloscope picture of amplifier (blue, top) and comparator output signals (green, second line from the top) of the HV2FEI4. The FE-14 hitbus signal is shown in pink (third line) and the injection signal from the GPAC in green (bottom).

is the sum of the output signal of all connected pixels. Thus, it increases with the number of pixels that are connected to the monitor output. This effect was confirmed and the measured values can be found in Table 6.3. In addition, the hitbus signal of the FE-I4 (pink) indicates, that the output signal of the HV2FEI4v2 is detected by the FE-I4.

\begin{tabular}{l|c|c|c|c|c} 
Fraction of pixels & 1 & 0.5 & 0.25 & $\frac{1}{12}$ & 0 \\
\hline Monitor output amplitude [V] & 1 & 0.8 & 0.4 & 0.3 & 0
\end{tabular}

Table 6.3: Amplitude of the monitor output signal for different fractions of pixels that are directed to this output. 


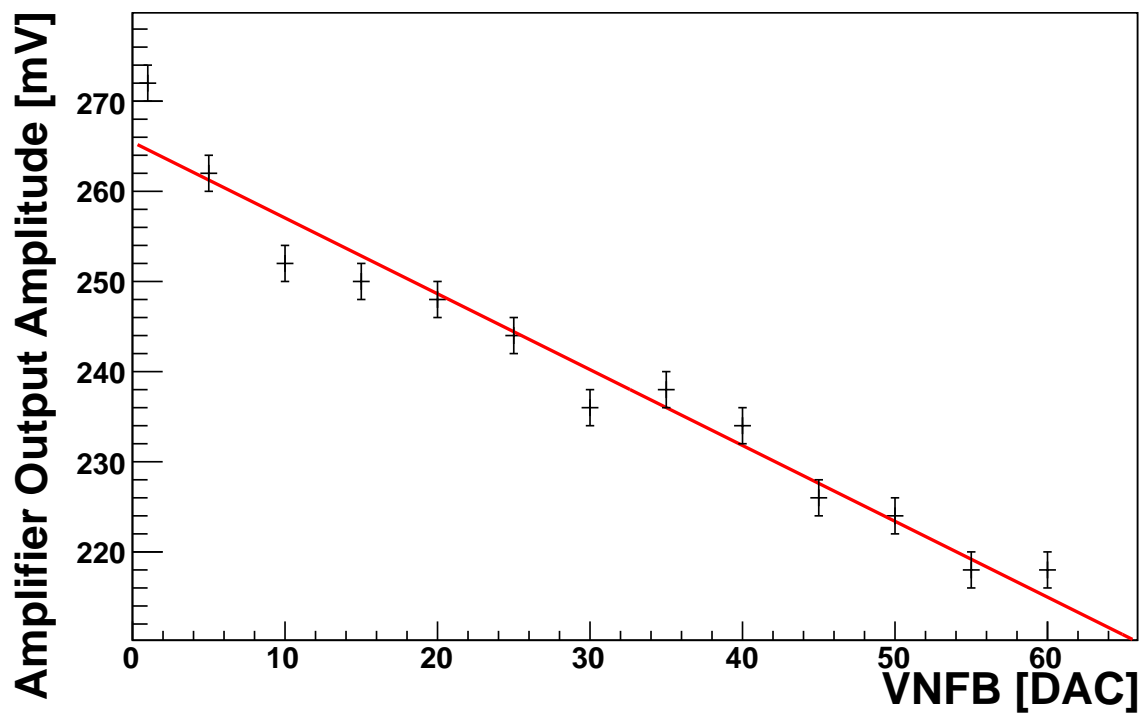

(a) Amplitude.

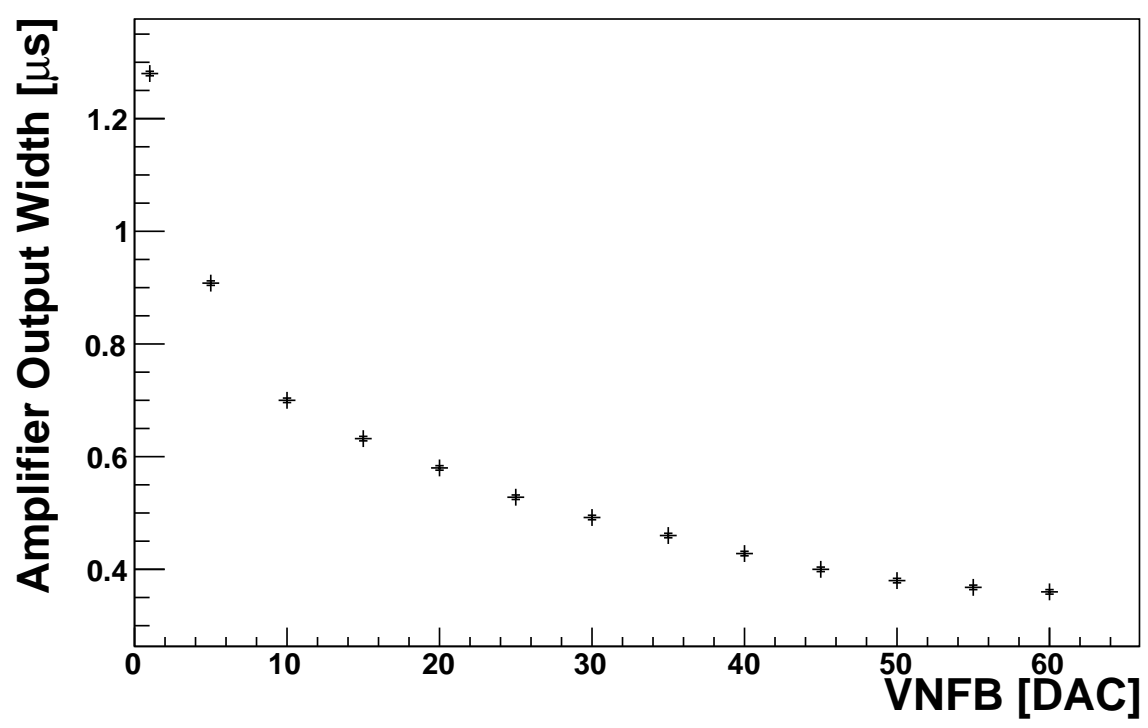

(b) Width.

Figure 6.9: Amplifier output signal of the HV2FEI4v2 for different VNFB DACs settings.

The shape of the amplifier output signal was investigated in more detail. The signal shape is influenced by the feedback parameter VNFB. On an oscilloscope the amplitude and the width of the amplifier output signal were investigated and measured for different $V N F B$ DACs. The injection voltage was $1.0 \mathrm{~V}$, which is expected to correspond to a charge significantly above threshold [68]. The result is shown in Figure 6.9. The amplitude (see 
Figure 6.9a) decreases linearly with increasing VNFB, whereas the width (see Figure 6.9b) decreases faster than linear. From this measurement it follows that the signal shape is not triangular, as already seen in Figure 6.8. Consequently, the relation between amplifier output amplitude and the charge from the initial particle is not linear, but more complex (see Section 3.4).

Subsequently, the time walk, which is the time between injection pulse and amplifier output signal of the HV2FEI4v2 sensor, is determined for various injection voltages. The delay caused by time walk is determined by the time between the falling edge of the injection signal and rising edge of the amplifier output signal over a certain trigger condition, as it is illustrated in Figure 6.10. The delay was measured for injection voltages between

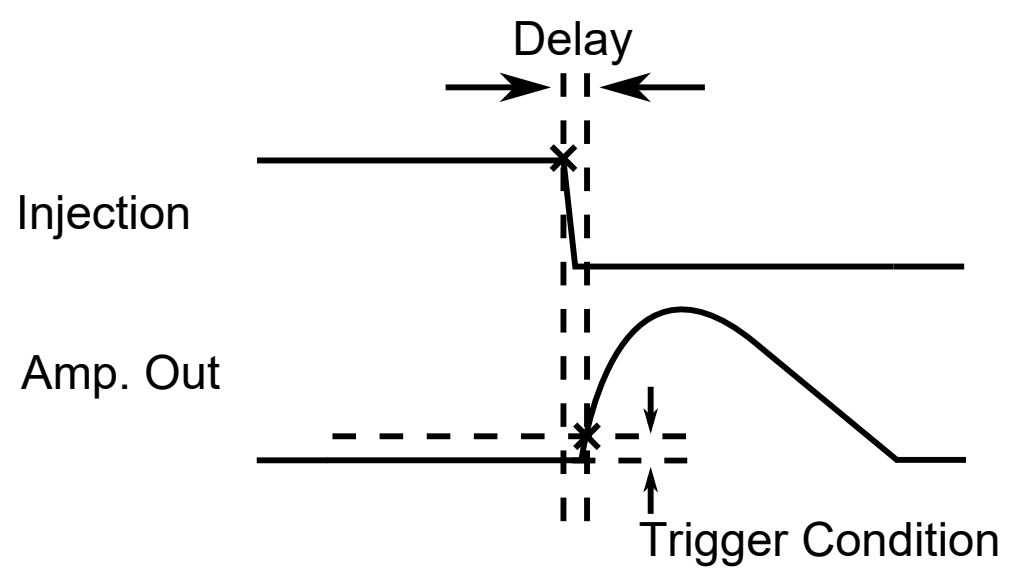

Figure 6.10: Schematic drawing of the time walk measurement of the HV2FEI4V2 amplifier output. The delay is the time between the fall of the injection signal and the rise of the amplifier output signal over a certain trigger condition.

$0.2 \mathrm{~V}$ and $1.0 \mathrm{~V}$. The trigger condition for the amplifier output signal was chosen to be $50 \mathrm{mV}$ to be well above the fluctuation of the baseline signal. Figure 6.11 shows the result of the time walk measurement. The delay decreases with increasing injection voltage. The largest injection voltage has a delay of approximately $35 \mathrm{~ns}$. The red line is at a delay of $60 \mathrm{~ns}$, which marks the $25 \mathrm{~ns}$ time window with respect to the largest injection voltage. Consequently, all injection voltages between $0.3 \mathrm{~V}$ and $1 \mathrm{~V}$ induce an amplifier output within 25 ns.

\subsubsection{Validation of the STcontrol Implementation}

To further test the STcontrol implementation of the functionalities of the CCPD module, consisting of the HV2FEI4v2 sensor and the FE-I4B readout chip, the result of the Analog Test CCPD (see Section 5.2.4) was investigated. Many parameters of the sensor and the readout chip needed to be adjusted, such as timing parameters (trigger delay and latency), sensor parameters, injection parameters and mask settings. Only if the combination of all parameters is correct, the result of the Analog Test CCPD is correct. 


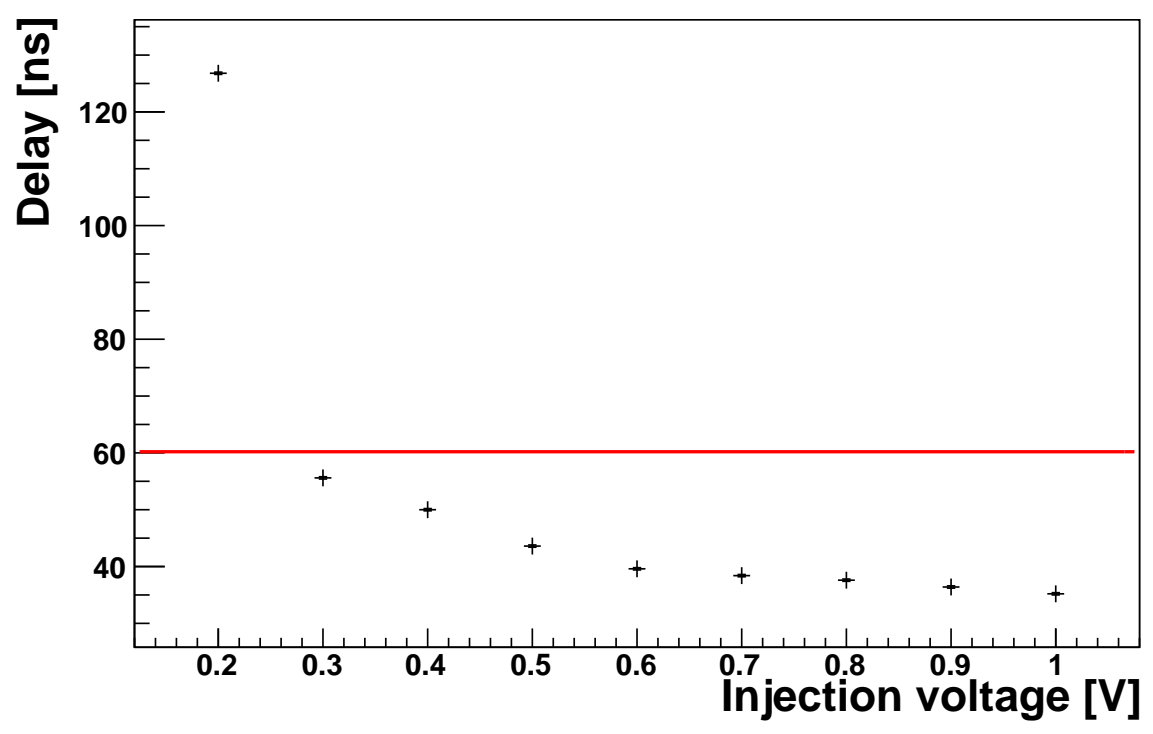

Figure 6.11: Delay between the injection signal and the amplifier output signal to estimate the time walk of the HV2FEI4v2 amplifier. The red line marks a delay of $60 \mathrm{~ns}$.

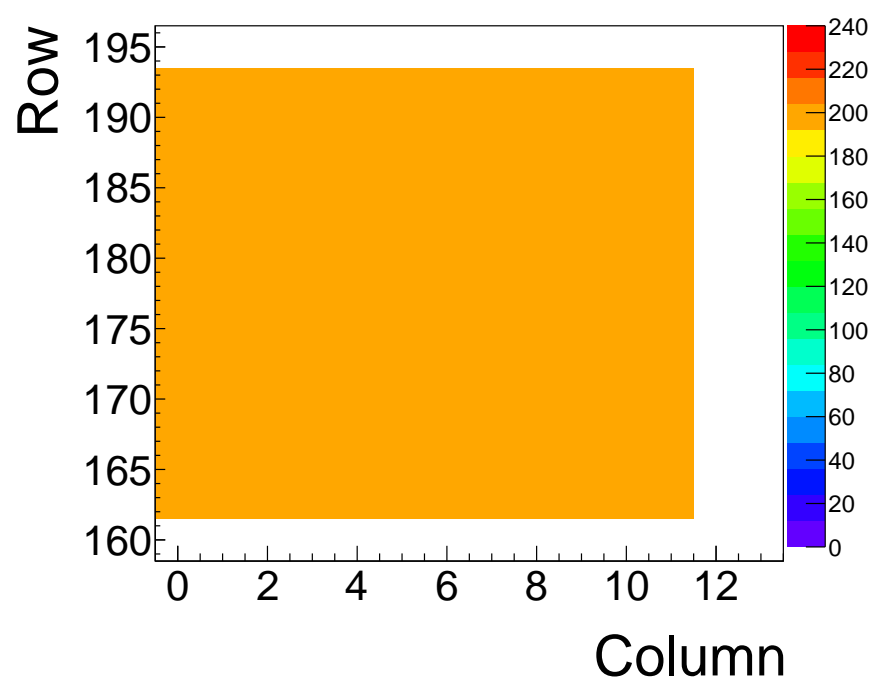

Figure 6.12: Result of an Analog Test CCPD. All pixels register 200 hits as expected.

Figure 6.12 shows the successful result of an Analog Test CCPD with 200 injections by the GPAC. The injection voltage was $1.0 \mathrm{~V}$, which is again expected to correspond to a charge significantly above threshold [68]. All FE-I4 pixels in columns 0 to 11 and rows 162 to 193 show exactly 200 hits. This result confirms, that the implementation of the HV2FEI4v2 sensor functionalities into STcontrol was successful, because it is the basis of 
all further calibration scans. All parameters are in a reasonable range, the interconnection via capacitive coupling is working and the charge that is injected into the sensor can be read out by the FE-I4.

In the previous test the injection voltage was high enough to produce a hit with a signal amplitude well above the HV2FEI4 threshold. In Figure 6.13, the result of the measurement of the occupancy for different injection voltages is shown. Figure 6.13a shows a pixel map with the accumulated occupancy for all injection voltages. It is clearly visible in the pixel map that the type A2 pixels (red, top) have a higher accumulated occupancy compared with the type A pixels (green, bottom). In Figure 6.13b the occupancy versus the injection voltage can be seen for one pixel of type A2. At an injection voltage of approximately $0.55 \mathrm{~V}$ the FE-I4 registers all 100 injections, which were injected into this one pixel of type A2. For one type A pixel (see Figure 6.13c), all 100 injections are seen at an injection voltage of $1.3 \mathrm{~V}$.

The plots of the occupancy versus the injection voltage are similar to Figure 5.2, which shows the measurement of the FE-I4 threshold. The two plots of the example pixels (6.13b and 6.13c) illustrate the principle of the threshold measurement based on the Analog Test $C C P D$. Using an S-curve fit the injection voltage at the HV2FEI4 threshold is determined, which will be investigated in the next section.

\subsubsection{HV2FEI4 Comparator Threshold Studies}

The HV2FEI4v2 comparator threshold is mainly controlled by the external threshold voltage $T h$. In this section, the influence of other parameters on the threshold is studied, using the Threshold Scan CCPD (see Section 5.2.4).

The result of the Threshold Scan CCPD is shown in Figure 6.14. During the scan, the injection voltage was varied from $0 \mathrm{~V}$ to $1.5 \mathrm{~V}$ in 61 steps. For every step, the occupancy per pixel was determined, and the resulting points were fitted with an S-curve fit. From the fit the injection voltage at the comparator threshold, the so-called threshold equivalent voltage $\left(\mathrm{Th}_{\mathrm{EV}}\right)$ and its equivalent noise threshold (ENV) are determined. The distribution of the $\mathrm{Th}_{\mathrm{EV}}$ can be seen in Figure 6.14a and its ENV distribution in Figure 6.14b. For the standard parameters (see Tables 6.1 and 6.2), the $\mathrm{Th}_{\mathrm{EV}}$ for the type A2 pixels is approximately $0.25 \mathrm{~V}$ with a dispersion of $0.03 \mathrm{~V}$ and the ENV is $0.02 \mathrm{~V}$ with a spread of $5 \mathrm{mV}$. It can be seen that the type A pixels have a higher $\mathrm{Th}_{\mathrm{EV}}$ and ENV voltage. The threshold-to-noise ratio is 6.25 , which yields a small possibility to measure a hit caused by noise.

So far, the threshold is given in units of voltage and not as it is common in units of elementary charge. A rough estimate for a calibration of these voltages into a charge was suggested by the chip designer [68]. The signal amplitude of an Fe-55 source was measured to be approximately $V_{\mathrm{Fe}}=150 \mathrm{mV}$. The gamma energy of Fe-55 is ca. $6 \mathrm{keV}$ [69], which corresponds to a charge of $Q_{\mathrm{Fe}}=1660 \mathrm{e}$ in silicon. The calibration of the threshold 


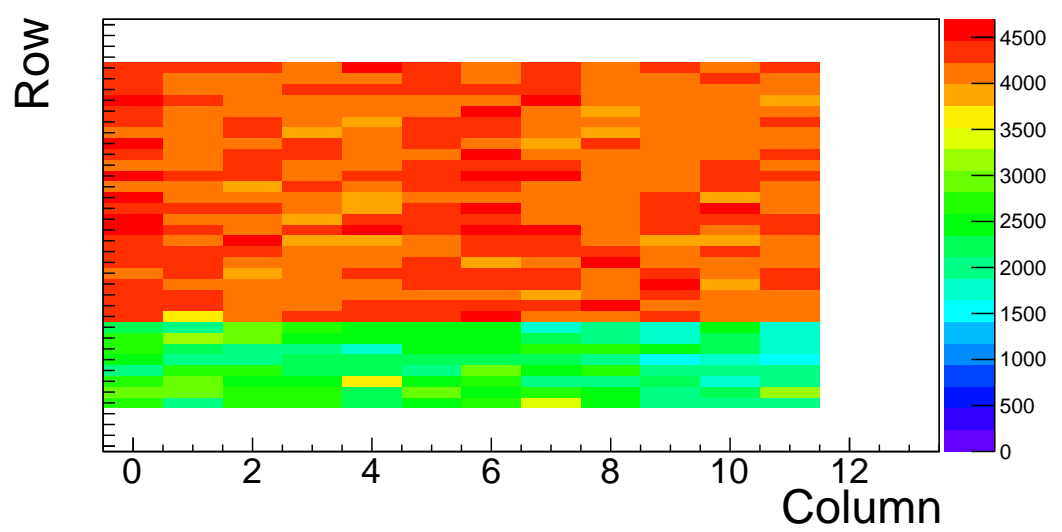

(a) Accumulated occupancy.

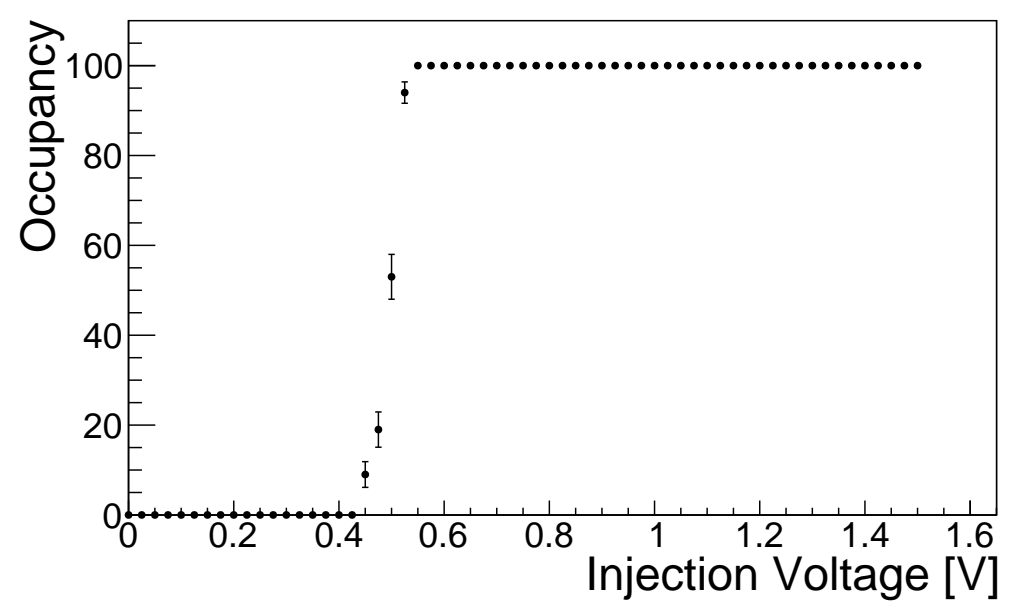

(b) Type A2.

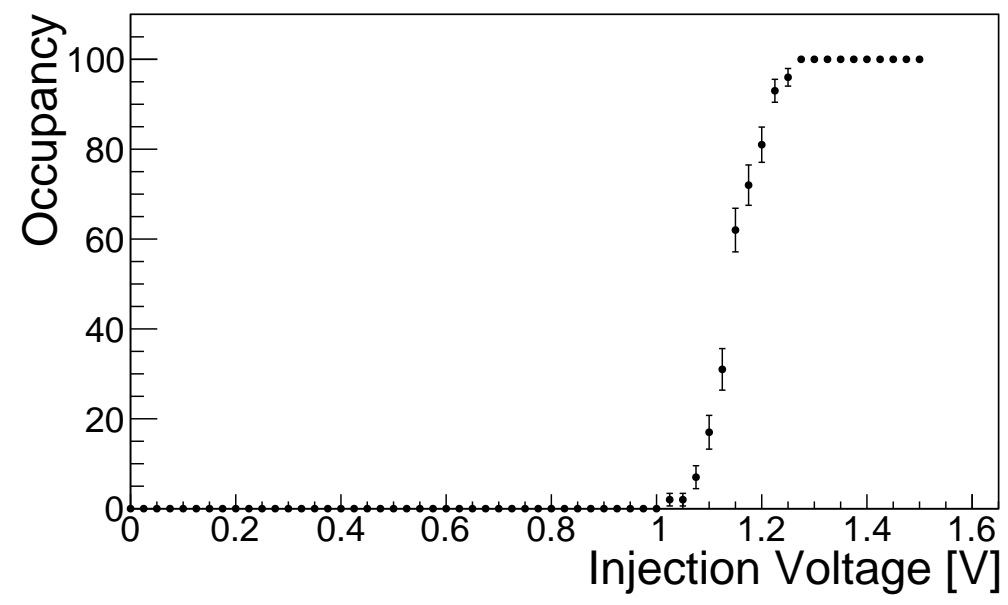

(c) Type A.

Figure 6.13: Hit occupancy for different injection voltages. The top plot shows a pixel map with the accumulated occupancy for all injection voltages. The middle and bottom plots show the occupancy versus the injection voltage for one pixel. 

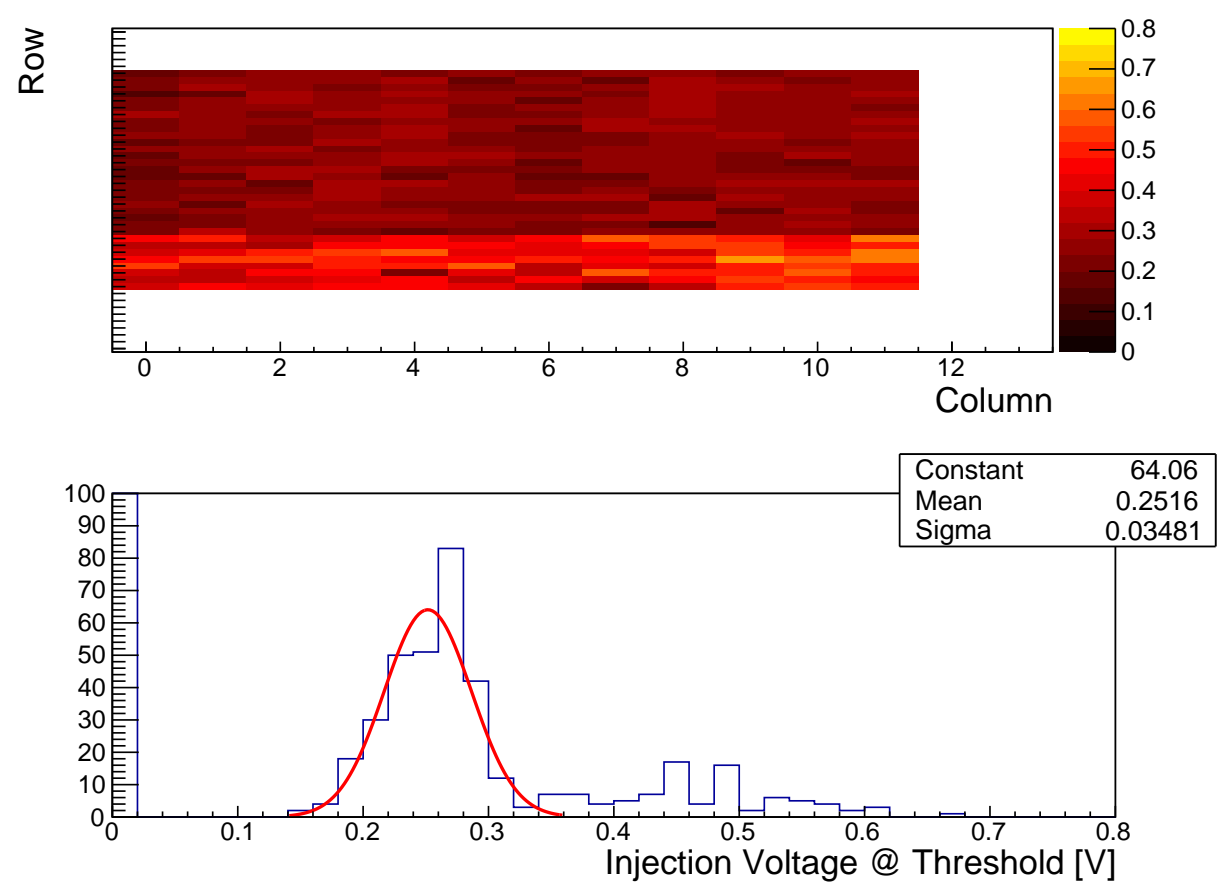

(a) Equivalent threshold voltage.
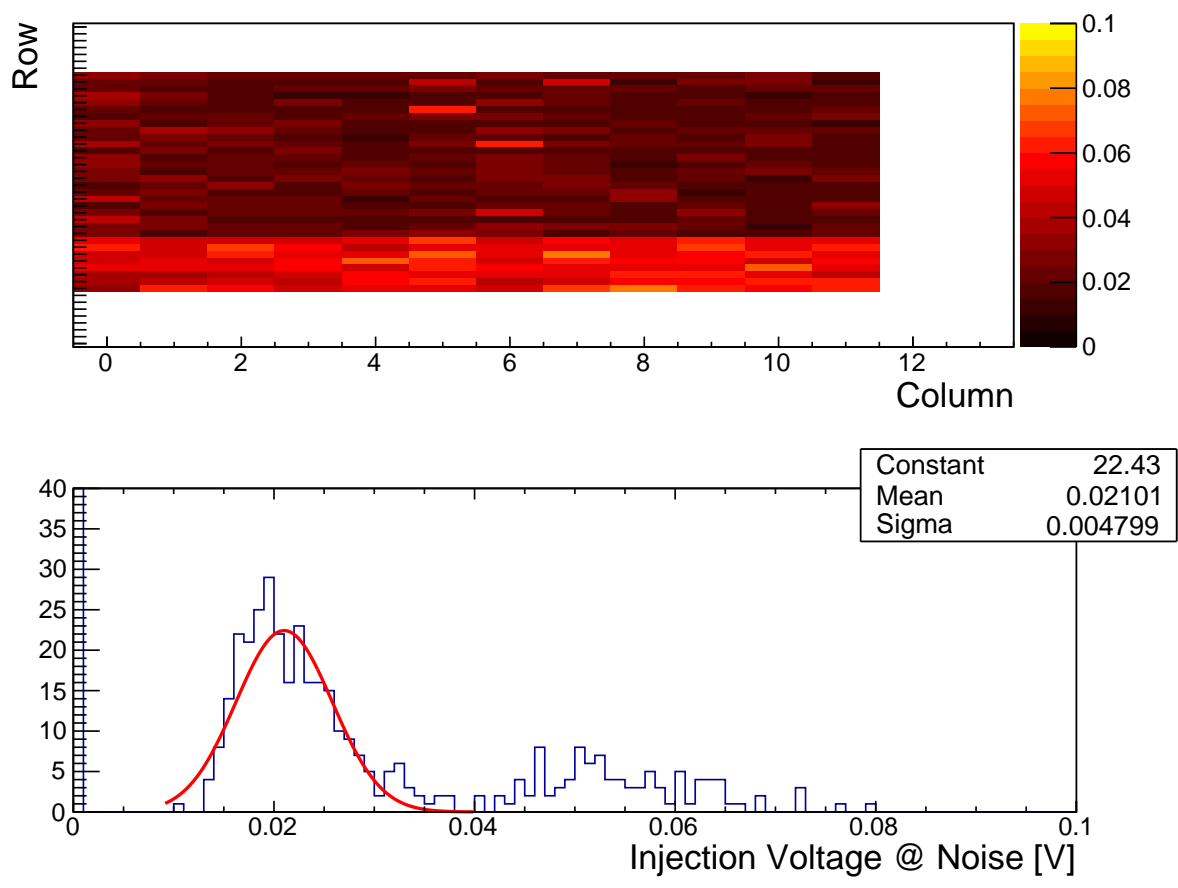

(b) Equivalent noise voltage.

Figure 6.14: Distribution of the injection voltages at the comparator threshold for the HV2FEI4V2 and the equivalent noise voltage. The top plots show a pixel map and the bottom plots all pixel values in one histogram. 


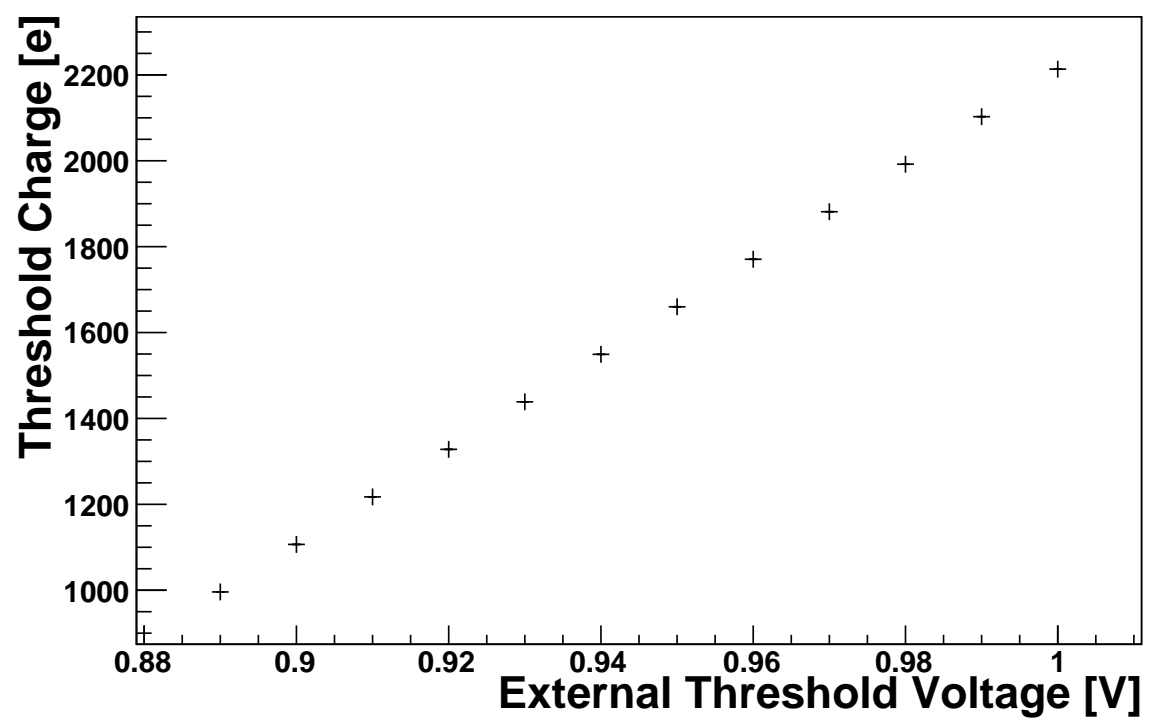

Figure 6.15: Calibrated threshold charge of different external threshold voltages, assuming a linear relation.

in equivalent charge $Q_{\mathrm{Th}}$ is

$$
Q_{\mathrm{Th}}=\frac{T h-B L}{V_{\mathrm{Fe}}} Q_{\mathrm{Fe}}
$$

Figure 6.15 shows the resulting threshold charge as a function of the external threshold voltage. This calibration assumes a linear behaviour of the charge as a function of the voltage, which is not correct as described in Section 6.2.2. Therefore, this calibration can only be used as a rough estimate of the charge. In the following, the threshold will be given in units of the injection voltage $\left(\mathrm{Th}_{\mathrm{EV}}\right)$.

The external threshold voltage is the parameter that influences the comparator threshold the most. Figure 6.16 shows the results of different Threshold Scans CCPD for external threshold voltages between $0.89 \mathrm{~V}$ and $1 \mathrm{~V}$. For voltages below $0.89 \mathrm{~V}$ no measurement was possible, because for some pixels the threshold was so low that only noise hits were registered. The $\mathrm{Th}_{\mathrm{EV}}$ increases with increasing external threshold voltages. The dispersion of the threshold increases with the external threshold voltage. In addition, the $\mathrm{Th}_{\mathrm{EV}}$ is measured for three values of the ThRes DAC. This parameter was not found to have the expected influence on the $\mathrm{Th}_{\mathrm{EV}}$.

As a next step the influence of the feedback parameter VNFB was studied. The measurement of the injection voltage at the comparator threshold for different external voltages and three VNFB DAC settings is shown in Figure 6.17. VNFB has a stronger influence on $\mathrm{Th}_{\mathrm{EV}}$ than the external threshold voltage. For large feedback currents the amplitude of the amplifier output signal is reduced, because part of the signal charge is compensated. This effect is known as ballistic deficit and leads to an increase of the effective comparator 


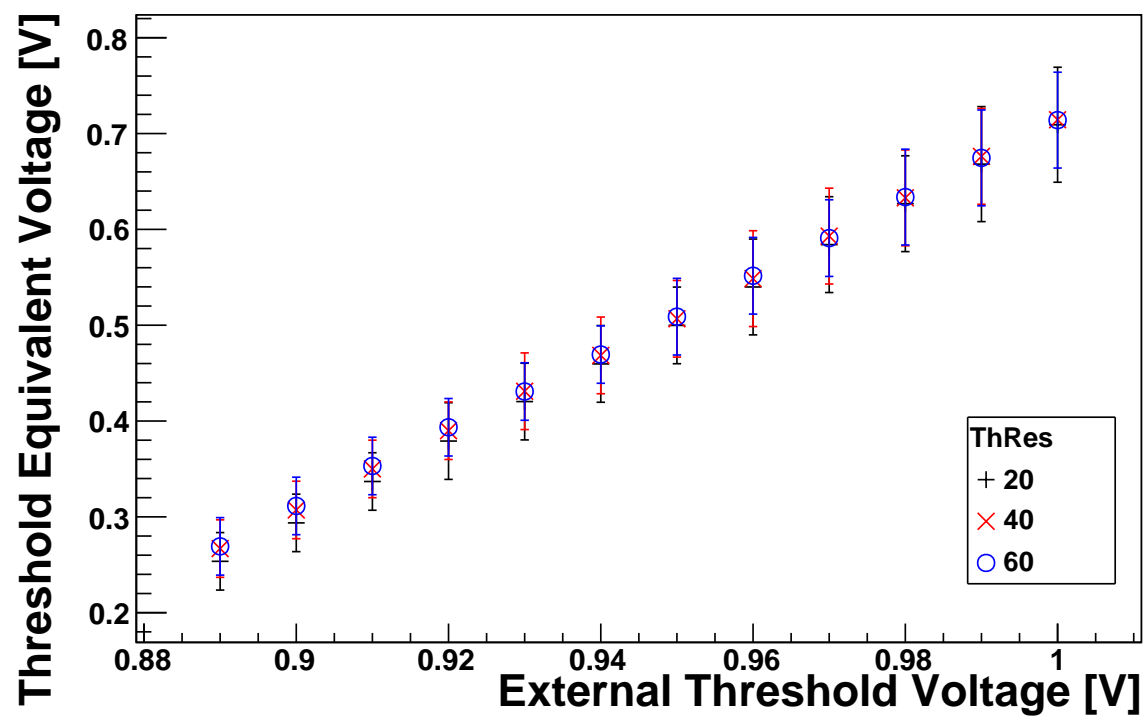

Figure 6.16: Injection voltage at the comparator threshold of the HV2FEI4v2 for different external threshold voltages and ThRes DACs values. The error bars show the pixel dispersion.

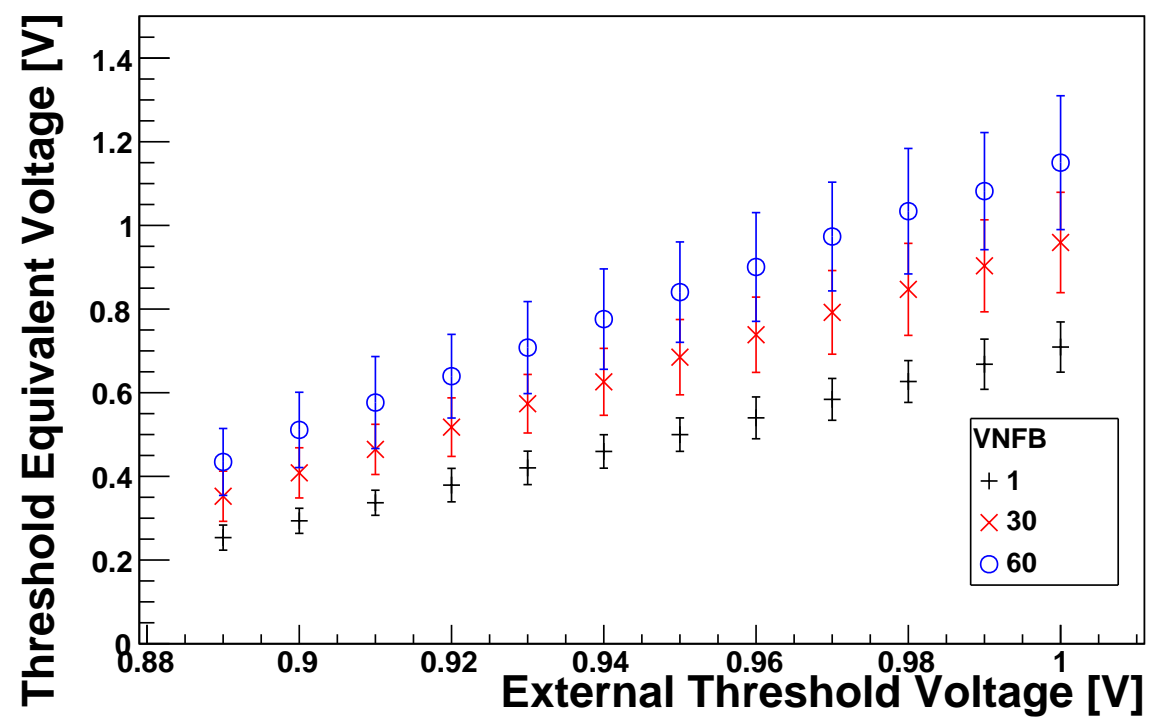

Figure 6.17: Injection voltage at the comparator threshold of the HV2FEI4v2 for different external threshold voltages and VNFB DAC settings. The error bars show the pixel dispersion. 
threshold at high values of $V N F B$.

Furthermore, the dispersion of the distribution increases with VNFB. This effect hints at the fact that the feedback transistors suffer more from process fluctuations than e.g. the comparator transistors.

Because the VNOut1/2/3 DACs will play an important role in the following studies, their influence on the comparator threshold was investigated. Figure 6.18 shows the measurement of the injection voltage at the threshold as function of the VNOut DAC values for all three subpixels. The error bars again indicate the pixel dispersion. The external threshold

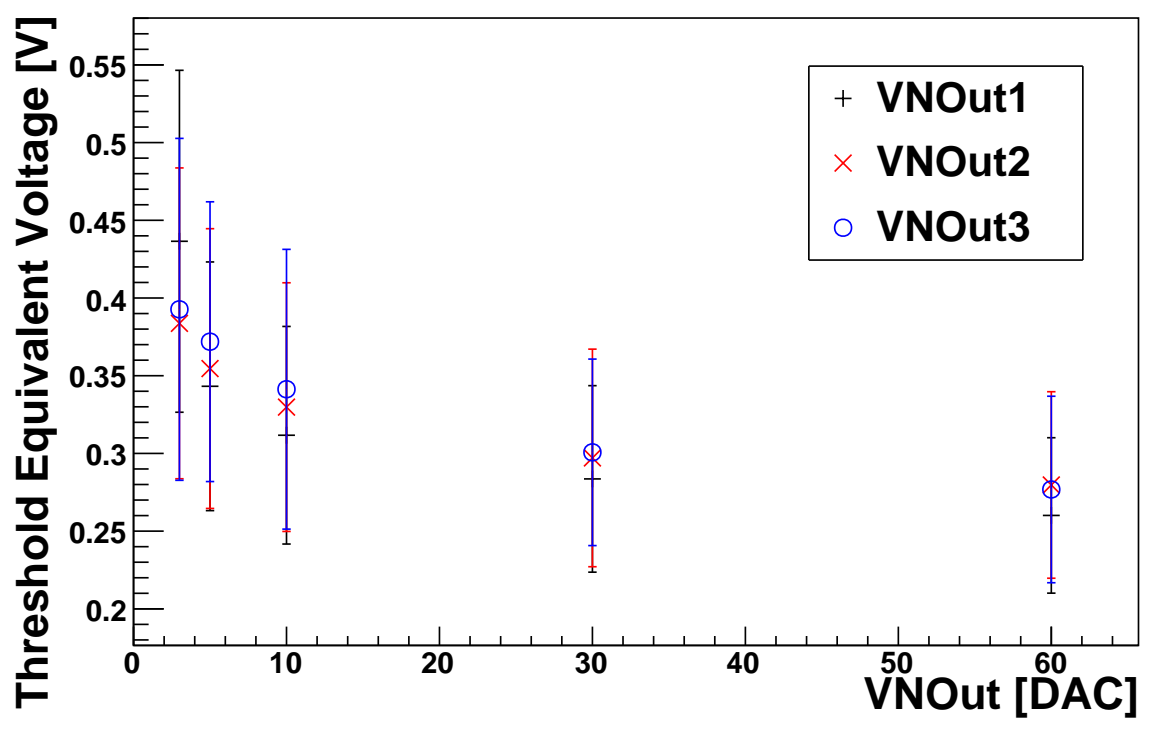

Figure 6.18: Injection voltage at the comparator threshold of the HV2FEI4v2 for different VNOut DAC settings. The error bars show the pixel dispersion.

voltage during the measurement was $0.89 \mathrm{~V}$. In the measurement for VNOut1 only the subpixel type 1 was enabled, as for the measurements with VNOut2 and VNOut3 only the subpixel types 2 and 3, respectively.

The VNOut1/2/3 DACs influence the threshold equivalent voltage. This effect was not expected, because the VNOut1/2/3 parameters are supposed to only influence the signal amplitude after the comparator (see Figure 6.3). No difference among the three subpixels was measured.

So far, only global parameters were studied for an influence on the comparator threshold. Using STcontrol, the InDAC, which adjusts the threshold per pixel, can be set for every pixel individually.

Figure 6.19 shows the result of the measurement of $\mathrm{Th}_{\mathrm{EV}}$ for different InDAC values. The error bars show again the pixel dispersion. The external threshold voltage is $1.0 \mathrm{~V}$. Two independent measurements (A and B) produce the same results.

For the measurement all pixels were set to the same InDAC value and a Threshold Scan 


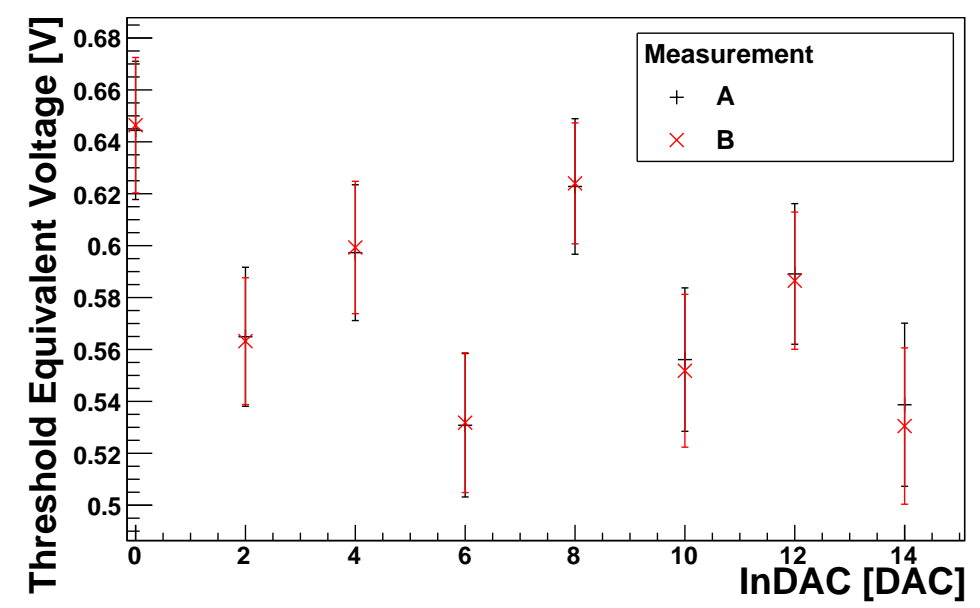

Figure 6.19: Injection voltage at the comparator threshold of the HV2FEI4v2 for different InDACs settings. The error bars show the pixel dispersion. Two independent measurements ( $A$ and $B$ ) produce the same results.

$C C P D$ was performed. The measurement shows that the InDAC does not influence the threshold in a well-defined way, which makes in-pixel tuning very difficult. With this result it was decided not to perform an in-pixel adjustment for the comparator threshold, which was supposed to reduce the dispersion, since no generally applicable tuning procedure could be defined.

\subsubsection{ToT Studies towards Subpixel Decoding}

For a successful subpixel decoding, the ToT response of the FE-I4 needs to be adjusted to the amplitude of the sensor signal. Before tuning the signals of the subpixel types 1,2 and 3, the impact of the VNOut1/2/3 DACs on the ToT is investigated.

Only one subpixel type was enabled at a time and the ToT response to different VNOut settings was measured. The same was repeated for all three subpixel types. Figure 6.20 shows the ToT response as a function of VNOut1/2/3 DAC values. The ToT $T_{\text {Code }}$ saturates for VNOut DAC settings above 12 and hence the output voltage of the HV2FEI4v2. Consequently, the parameter range is small, in which a significant change of the output voltage is possible. In this range the response of the different subpixel types varies up to $2 \mathrm{ToT}$ units. VNOut2 has the highest ToT response. For VNOut1/2/3 DAC settings above 12 the ToT response is at its maximum. Consequently, the available range to separate the subpixels is small.

Considering the result above, the target value of the FDAC Tune CCPD was chosen to be $T o T=3$ for a signal with $V N O u t 1=3$ to get the lowest possible $T o T$ response. During the tuning only the subpixel type 1 was enabled. The FDAC distribution, which is the result of the tuning, is shown in Figure 6.21. The FDAC distribution (see Figure 6.21b) is broad, which means that the differences among the pixels are large and the FDAC setting 


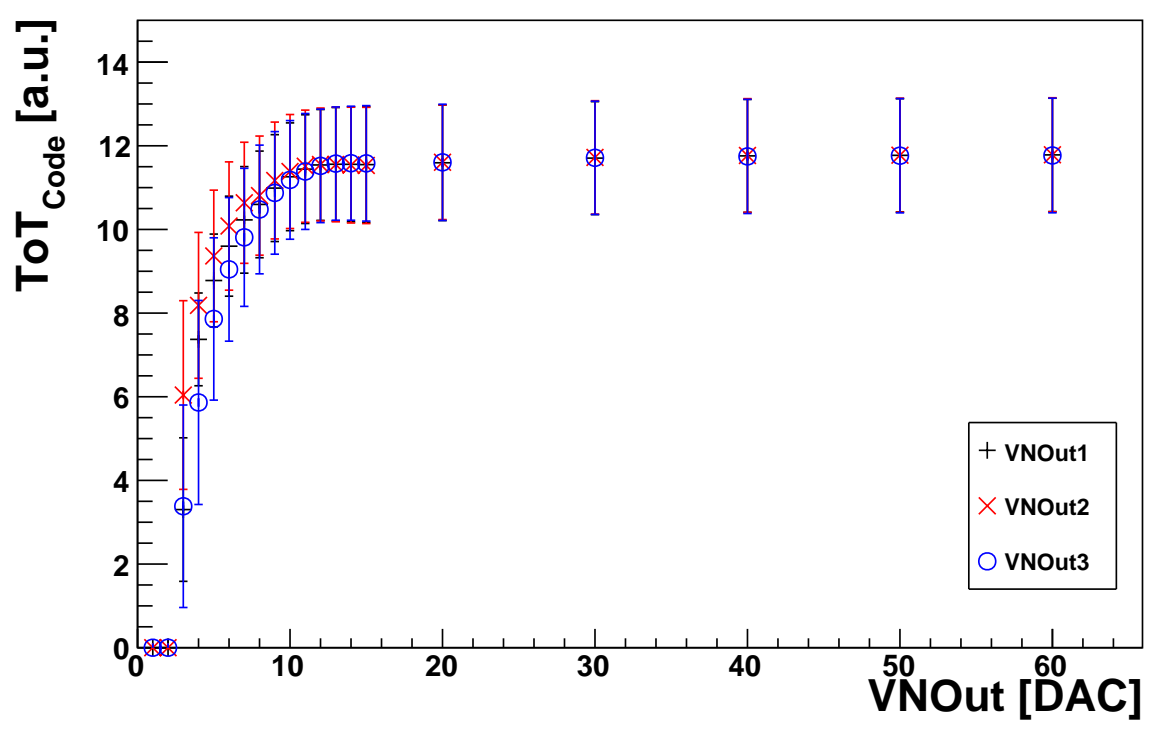

Figure 6.20: TOT response as a function of VNOut1/2/3 DACs for the three subpixel types for a given feedback tuning of the FE pixels. The error bars show the standard deviation of the distribution.

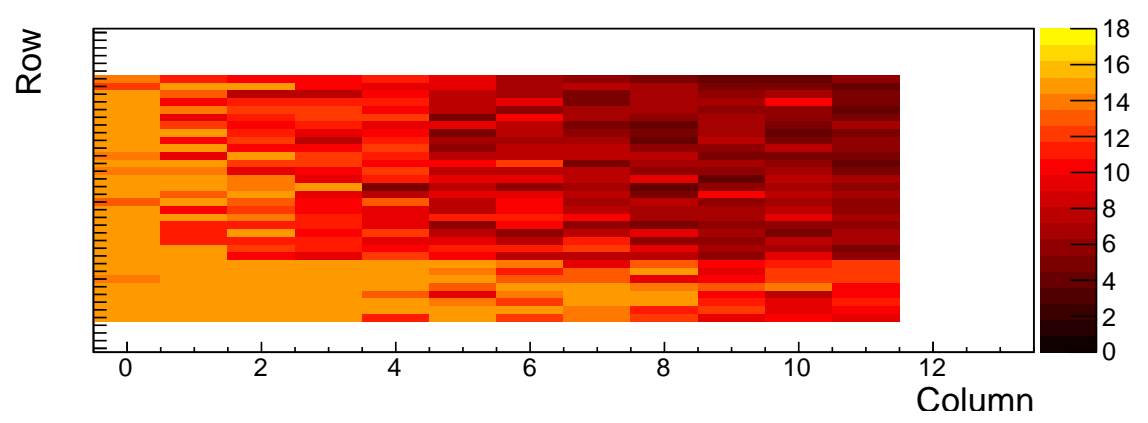

(a) FDAC Pixel Map.

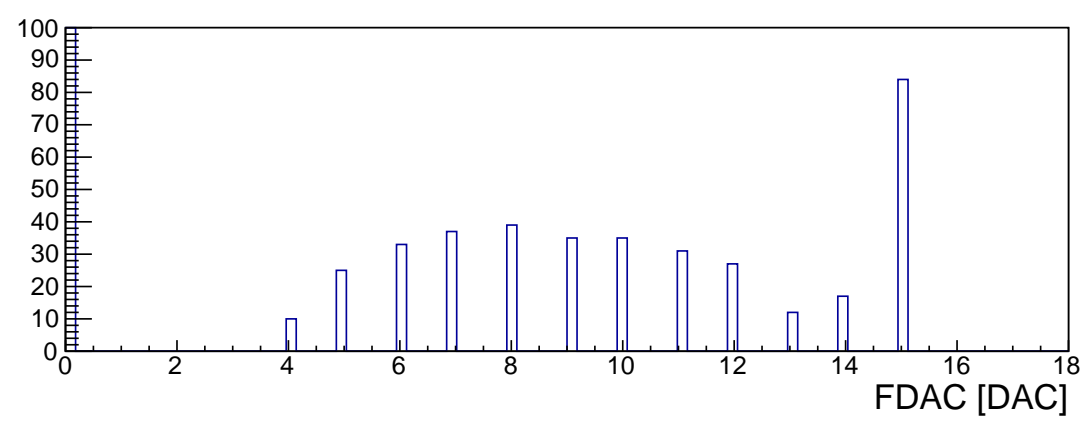

(b) Histogram.

Figure 6.21: Result of the FDAC Tune CCPD. The distribution of the FE-I4 FDAC values is shown as a pixel map (top) and a histogram (bottom). 
compensates this effect. The large peak at 15 contains the pixels, for which the FDAC cannot compensate the differences among the pixels. Furthermore, the FDAC map (see Figure 6.21a) reveals a gradient from the upper right corner to the lower left corner.

A possible explanation for the latter effect is that the glue layer between the readout chip and the sensor is not uniform in its thickness. Consequently, the coupling capacitance is different for the pixels. The signals received by the FE-I4 readout chip have different amplitudes and hence different ToT values.

To test this hypothesis, the height difference between the sensor surface and the readout chip surface was measured for six points on the HV2FEI4 sensor. Figure 6.22 shows the measured height differences for six different positions. The height at every position was measured using a microscope, focussing on the surfaces. The difference between the focus positions yields the height difference. Each measurement was repeated five times. The height differences for position 3 and 4 are significantly smaller compared with the other positions with $258.0 \pm 1.3 \mu \mathrm{m}$ and $260.0 \pm 1.1 \mu \mathrm{m}$, respectively. At position 6 the

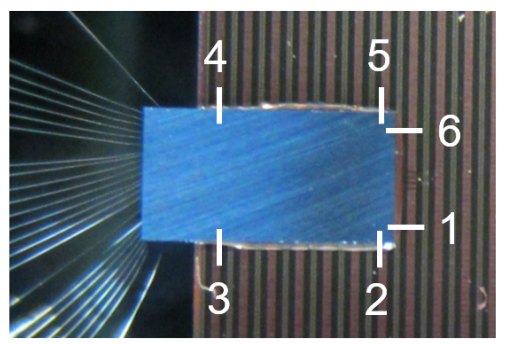

(a) Positions.

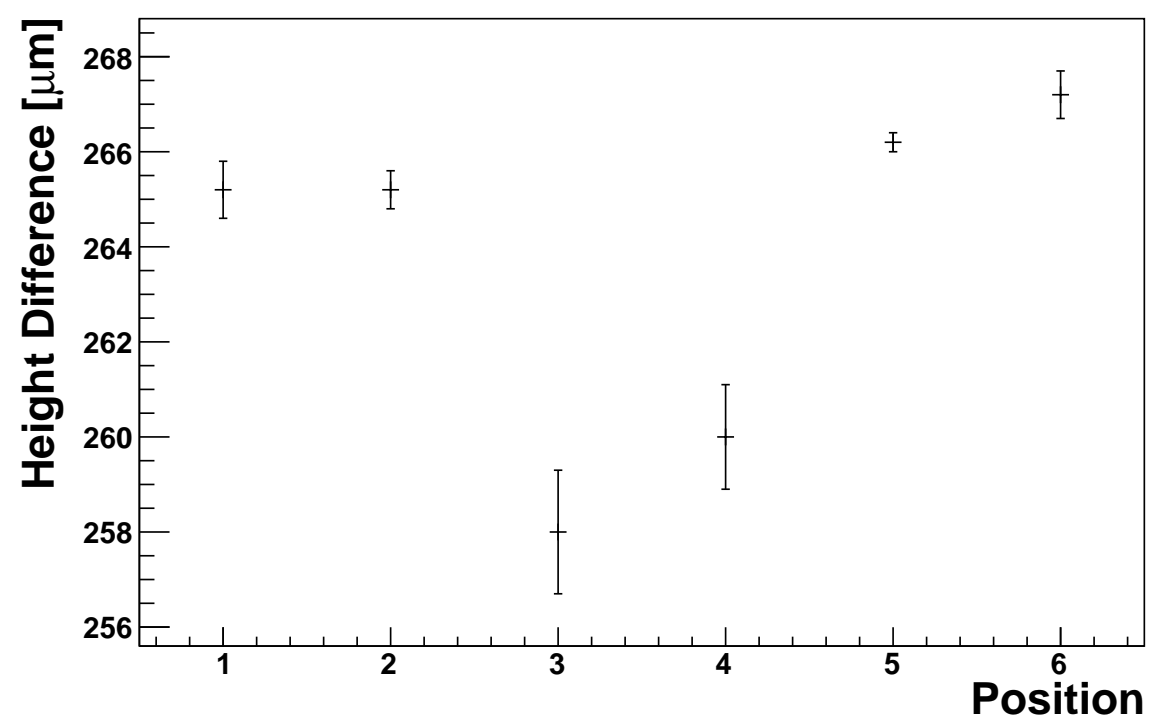

(b) Measurement.

Figure 6.22: Height difference between the sensor and the readout chip surfaces at six positions. 
height difference is $267.2 \pm 0.5 \mu \mathrm{m}$, which is the largest value. A difference in the glue layer thickness is a possible explanation for this effect. Furthermore, the sensor thickness itself can vary by a few micrometers.

Consequently, with the same output voltage, the signal that is received by the readout chip is higher in the lower left corner. Thus, the FDAC is set to higher values by the tuning, to increase the feedback current. But the FDAC reaches its dynamic range, which is visible in Figure 6.21a.

The FDAC values were tuned to $T o T=3$ for a signal with $V N O u t 1=3$ as described before. To get the largest possible separation between the ToT response of the three subpixels, the VNOut2 was set to 60, which results in the highest possible amplitude (see Figure 6.20). The VNOut 3 was set to 5 to get a medium ToT response.

With these VNOut1/2/3 settings an Analog Test CCPD was performed and the average ToT response per pixel was measured. Figure 6.23 shows the ToT distributions for the three subpixels after the tuning. Most of the subpixels can be separated from the others, but there is some overlap between the distributions.

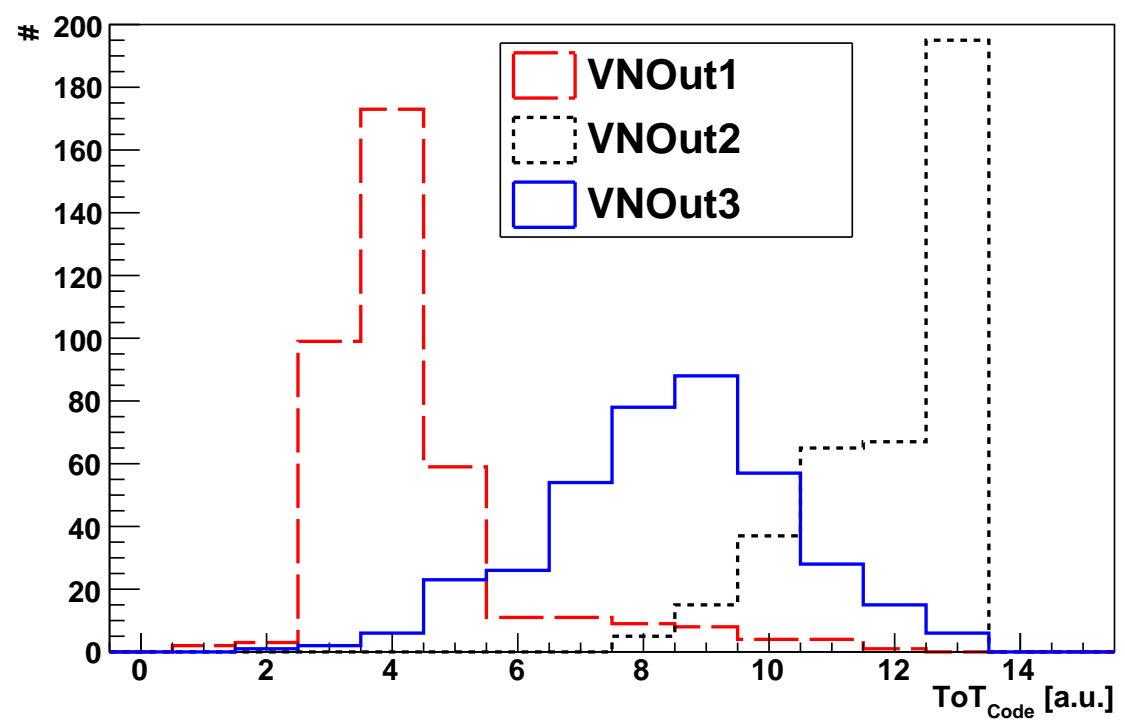

Figure 6.23: TOT distribution of the HV2FEI4V2 module for the three subpixel types measured in three separate Analog Test CCPD scans.

\subsubsection{Measurement}

The current-voltage characteristic of the HV2FEI4v2 sensor is shown in Figure 6.24. The leakage current of the module was investigated down to $-60 \mathrm{~V}$ at room temperature. No breakdown is observed down to this voltage.

The charging time of the capacitance of the sensor is longer than a minute. A stable leakage current is only reached after this minute. To accelerate the charging process a $1 \mathrm{M} \Omega$ resistance is put in parallel to the sensor for the measurement. The current-voltage 


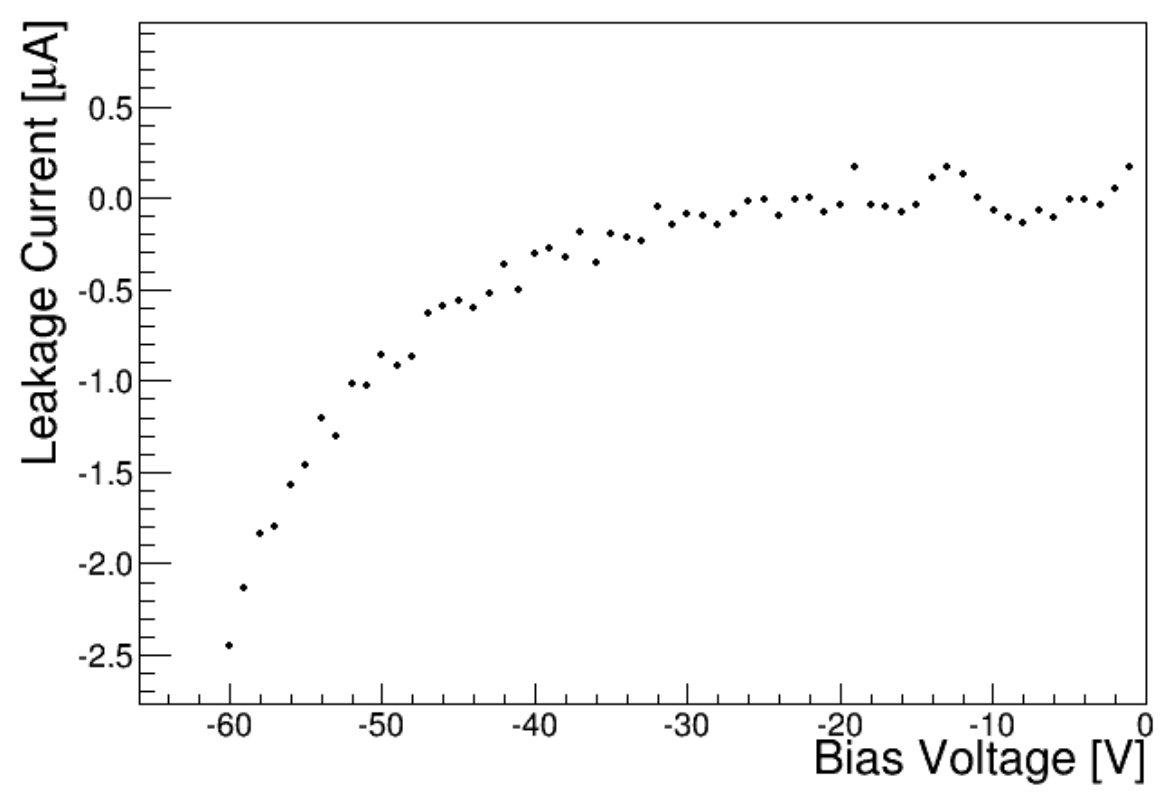

Figure 6.24: Current-voltage characteristic for the HV2FEI4V2 module. The errors bars are too small to be seen.

characteristic of the resistor is subtracted from the combined measurement of sensor and resistance to obtain the characteristic of the sensor only. To estimate the uncertainty on the current, the spread of the voltage drop over the circuit was measured over a time of $10 \mathrm{~ms}$. The uncertainty on the current measurement was calculated to be $3.5 \mathrm{nA}$. The maximum fluctuation range of the current is approximately $0.1 \mu \mathrm{A}$. The IV characteristic was only measured at one time. This measurement method explains the fluctuations in the current visible in 6.24 .

The leakage current is same order of magnitude $(\mathscr{O}(\mu \mathrm{A}))$ as the four-chip module (see Figure 4.9), which was not expected, because the area of the HV2FEI4v2 is less than 1\% of the area of the four-chip module.

\subsection{Hit Efficiency Studies with One Subpixel Type}

\subsubsection{Comparison of the Three Subpixel Types}

To study quantities such as the hit efficiency of the CCPD module, test beam measurements were performed at DESY in November 2015 with $4 \mathrm{GeV}$ electrons. The EUDET telescope was used for the measurements, the track reconstruction was performed in the EUTelescope software framework and track data were analysed with TBmonII (see Section 5.3).

The measurements were performed at room temperature, varying the sensor bias voltage and HV2FEI4 configuration parameters. Approximately $2 \cdot 10^{6}$ triggers were recorded per parameter setting. The statistical uncertainty on the hit efficiencies is less than $0.08 \%$ 
because of the large number of recorded tracks.

An IBL-like FE-I4 sample with a $200 \mu \mathrm{m}$ thick planar n-in-p sensor was used as reference plane. It is known from earlier test beam measurements, that the module has a uniform hit efficiency above 99\%. The CCPD sensor area is small compared to the area of the trigger scintillators. Hence, the reference module is used as region-of-interest trigger plane to only consider tracks in the CCPD sensor area.

At first, the telescope pointing resolution is determined from the residual distributions of the reference plane. The residual is defined as the difference of the hit and the track position, separately in $x$ - and $y$-direction. For the measurement only good tracks (as defined in Section 5.3.6) are considered that result in a hit in exactly one pixel. Figure 6.25 shows the residual distributions in $x$-and $y$-direction for the FE-I4 reference sample. The distributions are fitted with a convolution of a rectangular function and a Gaussian. To determine the telescope pointing resolution, the pixel width of the fit was fixed to $250 \mu \mathrm{m}$ in $x$-direction and $50 \mu \mathrm{m}$ in $y$-direction. The $\sigma$ of the fit is $10.2 \pm 0.2 \mu \mathrm{m}$ for the $x$-direction and $10.4 \pm 0.1 \mu \mathrm{m}$ for the $y$-direction. Consequently, the pointing resolution of the telescope is estimated to be approximately $10 \mu \mathrm{m}$.

The distribution is better described by a fit with a free pixel width. The pixel widths obtained from the fit are smaller than the expected widths. This effect is a result of the discriminator threshold of the readout pixels. Particles that hit a pixel at the edge produce a small fraction of the charge in the neighbouring pixel, which can be below threshold. Consequently, the hit position is considered to be further in the middle of the pixel than it really is.

For the first test beam studies with the CCPD module only one subpixel type was enabled at a time and the differences between the subpixel types were investigated. In the analyses the subpixel positions are identified as described in Section 6.4.1. The external threshold voltage was chosen to be $1.0 \mathrm{~V}$ and all InDAC values were set to 6 . Furthermore, the standard parameter settings (see Table 6.2) were used and a sensor bias voltage of $-40 \mathrm{~V}$ was applied.

For the following analyses only good tracks and matched hits, as defined in Section 5.3.6, were considered. The matching radius, in which the interpolated track position is associated with a hit in a pixel, is set to the pixel pitch plus $10 \mu \mathrm{m}$ to account for the telescope resolution. The matching radius in the reference plane equals twice the pixel pitch to consider effects due to multiple scattering. For the hit efficiency analyses only the type A2 sensor pixels were included in the results. In the other analyses the type A and A2 were studied.

With one subpixel type enabled during the measurements only one third of all pixels are enabled. Consequently, the number of pixels that are in a cluster is dominantly one. Clusters with a size of two pixels were found to be two orders of magnitude less likely.

Residual distributions for cluster size one, which are a measure for the pixel resolution, are presented for the CCPD module in Figure 6.26. The single pixel resolution, not considering threshold and charge sharing effects, is given for homogeneous hit probability across the pixel by $d / \sqrt{12}$ with the pixel pitch $d$. For the pixel sizes of the HV2FEI4v2, 


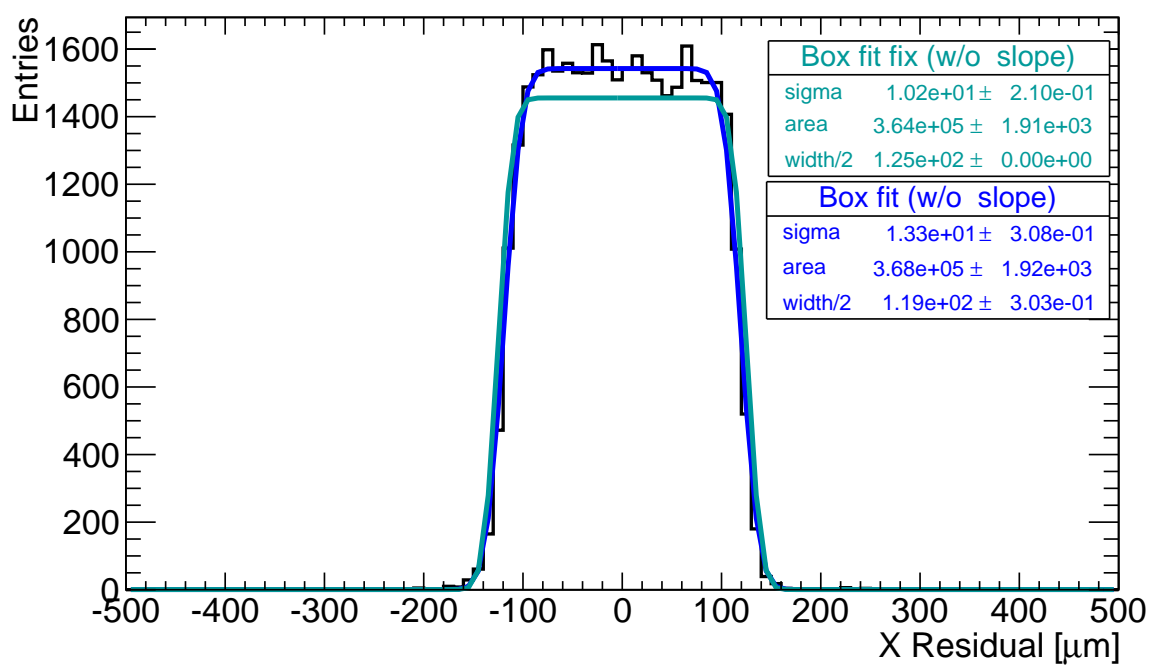

(a) $x$ residual.

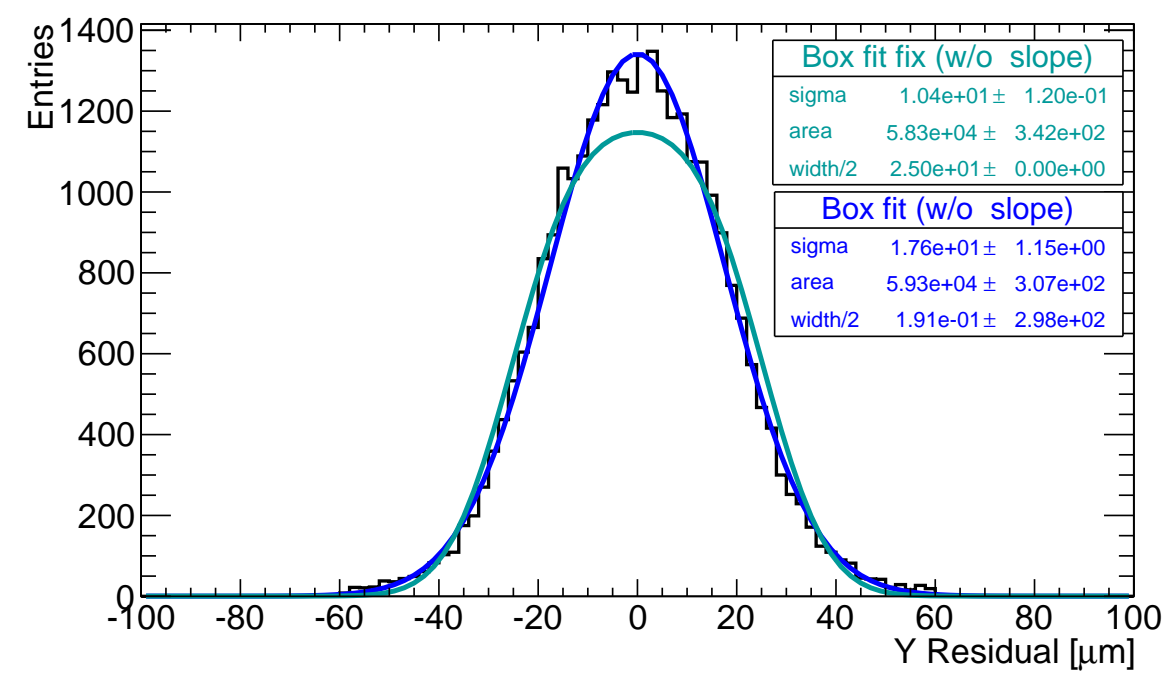

(b) $y$ residual.

Figure 6.25: Residual distributions for $x$ - and $y$-direction for the FE-14 reference sample. The distributions are fitted with a convolution of a rectangular function with a Gaussian. The difference between the fits is the pixel width parameter, which is free in one case (blue) and in the other fixed (green) to determine the telescope pointing resolution. 


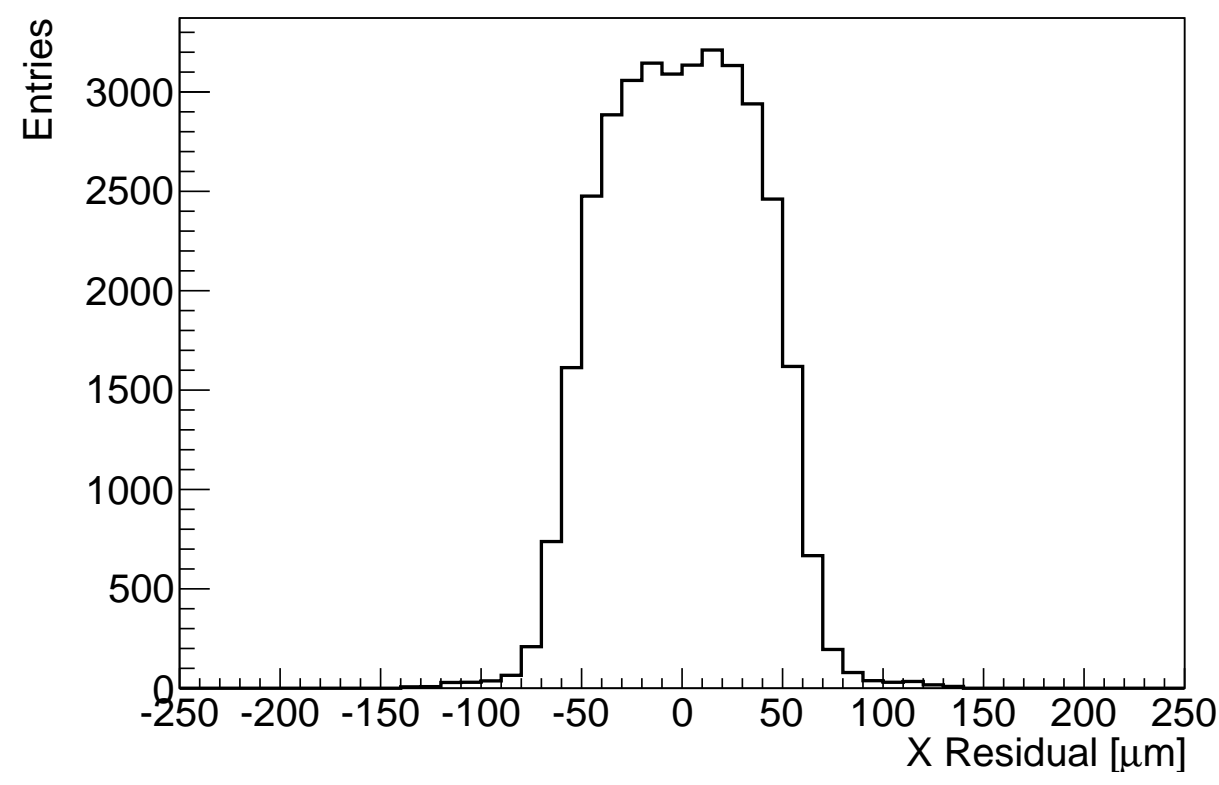

(a) $x$ residual.

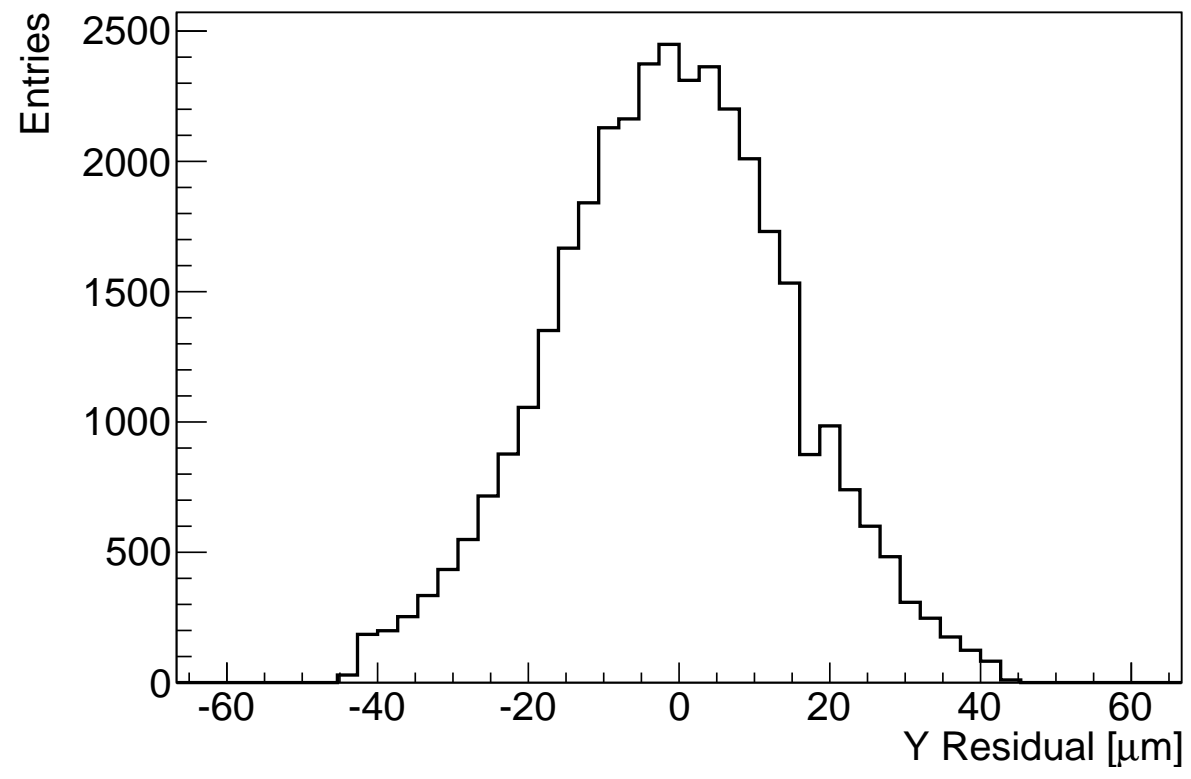

(b) y residual.

Figure 6.26: Residual distributions in $x$ - and $y$-direction of the CCPD module with the HV2FEI4V2 sensor. 
this value corresponds to

$$
125 \mu \mathrm{m} / \sqrt{12}=36.1 \mu \mathrm{m} \text { and } 33 \mu \mathrm{m} / \sqrt{12}=9.5 \mu \mathrm{m} .
$$

The standard deviation of the residual distribution is the quadratic sum of the spatial resolution of the HV2FEI4 and the pointing resolution of the telescope obtained using unbiased tracks, i.e. track fitting does not take the hit on the DUT into account. With the telescope resolution of approximately $10 \mu \mathrm{m}$, the expected values for the standard deviation are $37.5 \mu \mathrm{m}$ and $13.8 \mu \mathrm{m}$ for the $x$ - and $y$-direction, respectively. The standard deviations for the distributions in Figure 6.26 are $35.6 \pm 0.1 \mu \mathrm{m}$ for the $x$-direction and $15.65 \pm 0.06 \mu \mathrm{m}$ for the $y$-direction. These values differ from the expected values. The smaller value for the $x$-direction can be attributed to errors in the cluster position, because hits close to the edge of a subpixel are treated incorrectly, since the neighbouring subpixels are turned off. The larger value for the $y$-direction is caused by a misalignment during track reconstruction.

The ToT response of the subpixel types with the VNOut1/2/3 DAC values set to 60 were investigated. Figure 6.27 shows the normalised ToT distributions for the three subpixel types. No systematic difference is visible between the distributions of the three subpixel types. Because of the high VNOut settings, the ToT response peaks at the overflow value of 14. This bin includes all $T o T=14$ and higher ToT responses. Furthermore, the ToT distribution is very broad and with a tail down to $T o T=1$. As shown in Section 6.2.4, the $T o T$ tuning can be used to separate the ToT distributions of the three subpixel types.

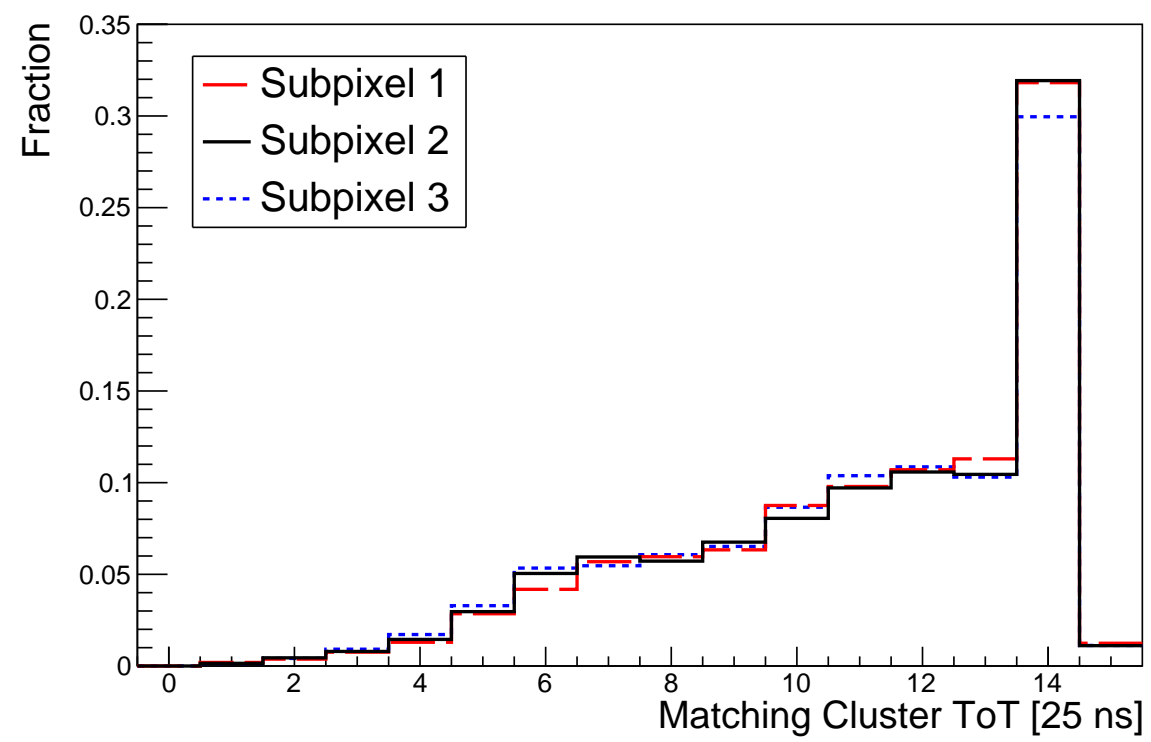

Figure 6.27: TOT distributions for three separate test beam measurements with only one enabled subpixel type, respectively. The VNOut1/2/3 DAC values are set to 60 . 


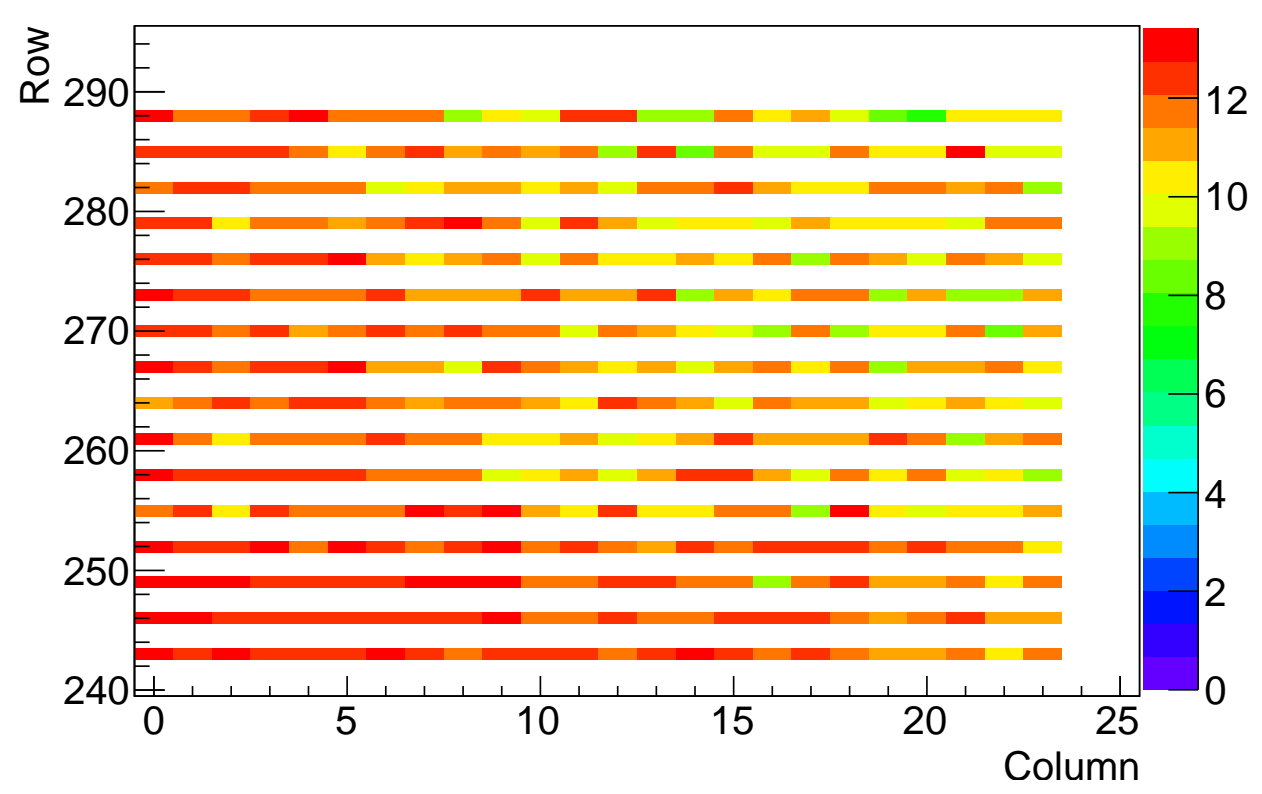

Figure 6.28: Pixel map of average cluster ToT for one enabled subpixel type. Only subpixel type 1 is enabled and the VNOut1 DAC value is set to 60 .

The normalised pixel map with the average ToT response per pixel for subpixel type 1 is shown in Figure 6.28. The ToT maps for the other two subpixel types look similar (see Appendix A.1 and A.2). A ToT gradient from the upper right to the lower left corner is visible. The average ToT response in the lower left corner is the maximum $T o T$ value. This effect is also seen in the FDAC map (see Figure 6.21), because FDAC reached its dynamic range and not all differences were compensated. It is likely caused by the height difference of the glue layer that connects the sensor and the readout chip, which results in a varying coupling capacity across the sensor.

The next studied quantity is the timing. Figure 6.29 shows the normalised Lvl1 distributions for the three subpixels, measured in three separate runs. Again no systematic difference between the three subpixel types is apparent. But the Lvl1 distributions are very broad compared to an FE-I4 module with a planar module (see Figure 4.12b). In the ATLAS Experiment only one Lvl1 bin is read out. Consequently, many hits in the HV2FEI4v2 module would not be registered. This broad timing distribution is further studied in Section 6.3.2.

As mentioned before, the external threshold voltage for these measurements was $1.0 \mathrm{~V}$. According to the charge calibration in Figure 6.15, this voltage corresponds to a threshold charge of approximately $2200 e$. The most probable charge deposited by a MIP-like particle in $10 \mu \mathrm{m}$ of silicon is ca. 1000e. Thus, only particles that deposit energy according to the tail of the Landau distribution can be detected. With a lower threshold charge higher hit efficiencies are expected. 


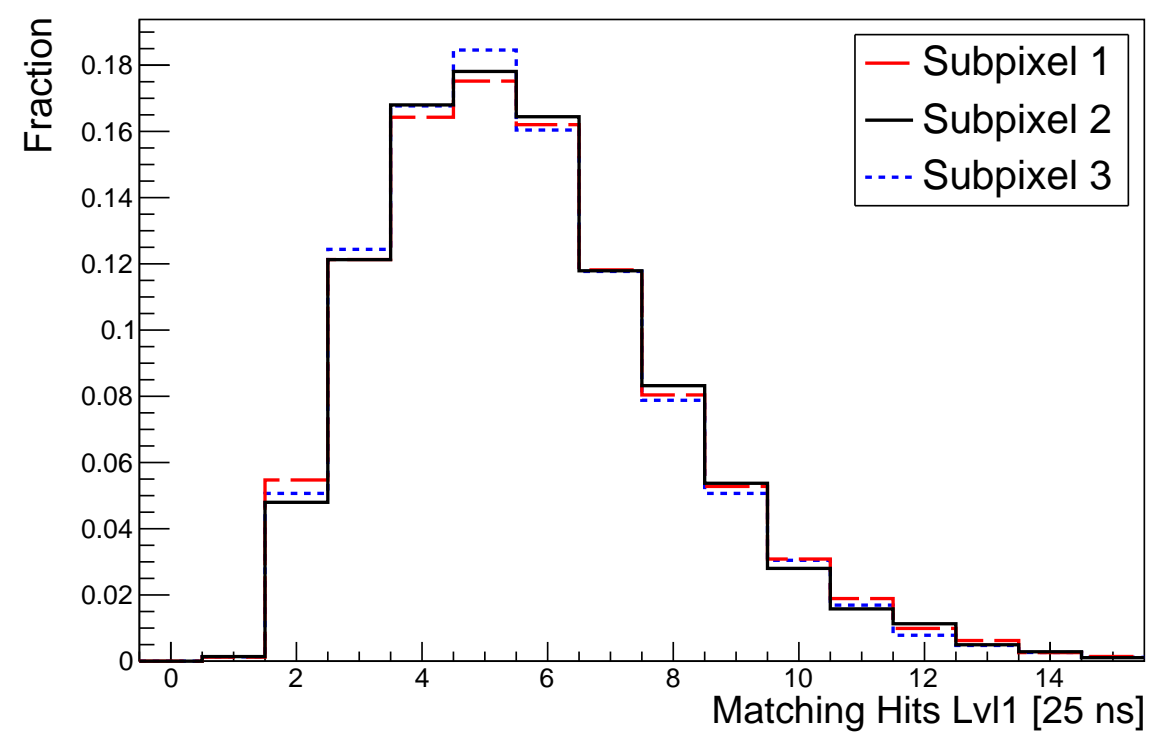

Figure 6.29: $L v / 1$ distributions for three separate test beam measurements with only one enabled subpixel type, respectively. The VNOut1/2/3 DAC values are set to 60 .

\begin{tabular}{c|c|c|c|c|c}
\multicolumn{2}{c|}{ Subpixel 1 } & \multicolumn{2}{c|}{ Subpixel 2 } & \multicolumn{2}{c}{ Subpixel 3 } \\
Efficiency & $\sigma$ & Efficiency & $\sigma$ & Efficiency & $\sigma$ \\
\hline 0.17 & 0.05 & 0.21 & 0.06 & 0.27 & 0.08
\end{tabular}

Table 6.4: Mean hit efficiencies and their standard deviation $\sigma$ for three separate test beam measurements with only one enabled subpixel type, respectively. The high HV2FEI4V2 comparator threshold needs to be taken into account, while looking at the hit efficiency values. See text for details.

Figure 6.30 shows the hit efficiency distributions for the three subpixel types from separate measurements. Differences in the three hit efficiency distributions are evident. Table 6.4 shows the mean values of the hit efficiency distributions and their standard deviation. Subpixel type 1 has the lowest mean hit efficiency, even with the same parameter settings as the other subpixel types. The upper limit of the hit efficiency distribution is 0.3 for subpixel type 1 . For subpixel type 2 the upper limit is at 0.4 . Subpixel type 3 has the highest mean hit efficiency and the upper limit of the hit efficiency of 0.55 , even though the standard deviation of subpixel type 3 is the largest. These low hit efficiencies are due to the high threshold charge of the comparator.

Figure 6.31 shows the hit efficiency pixel map for subpixel type 1. A uniform hit efficiency over the sensor area can be seen. The pixel maps for the other pixel types also show a uniform hit efficiency (see Appendix A.3 and A.4).

The signal amplitudes of the three subpixel types are identical (see Figure 6.20) as well 


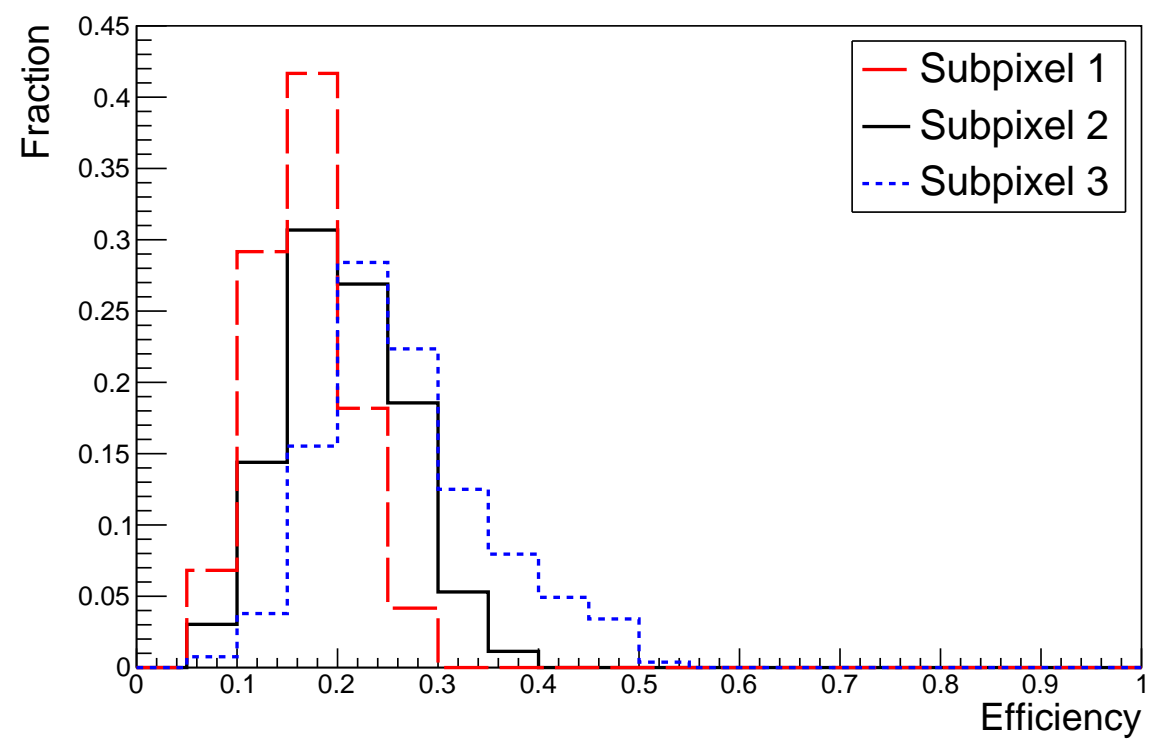

Figure 6.30: Hit efficiency distributions for three separate test beam measurements with only one enabled subpixel type, respectively. The external threshold voltage is $1.0 \mathrm{~V}$.

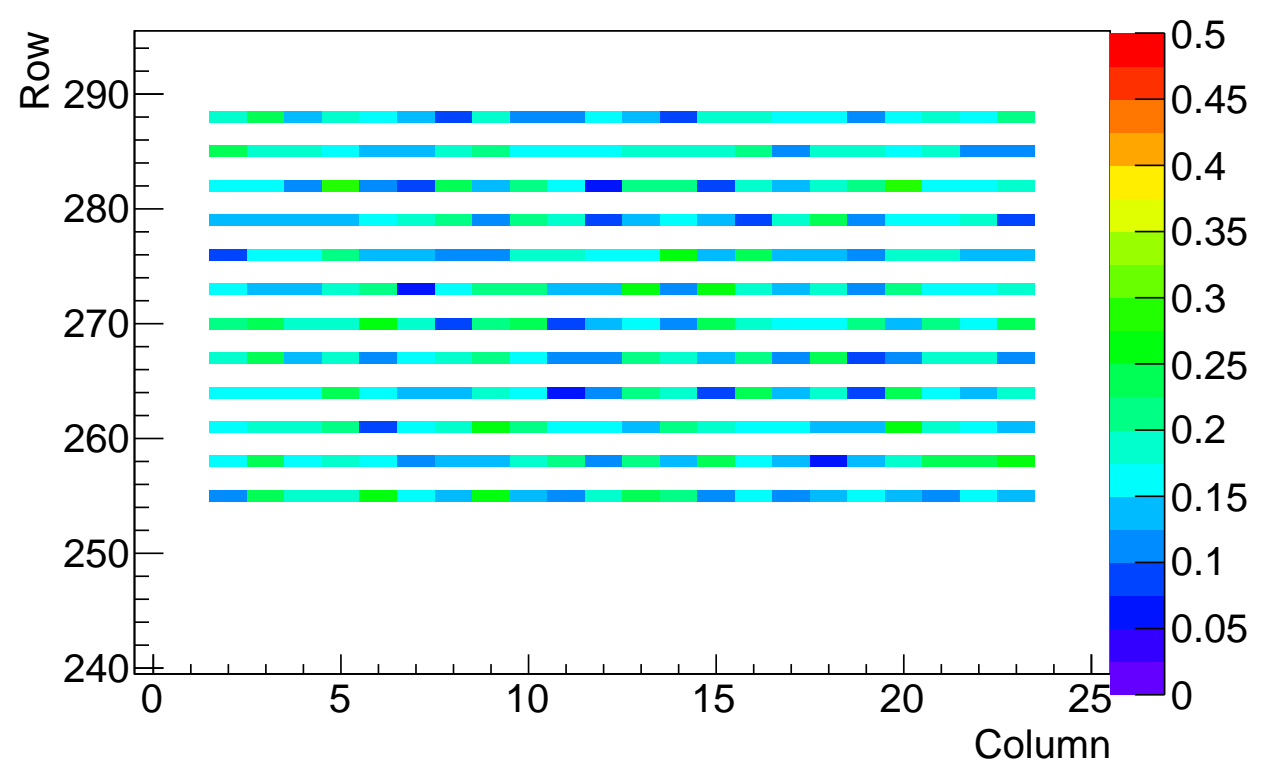

Figure 6.31: Hit efficiency pixel map for one enabled subpixel type. Only subpixel type 1 is enabled. The external threshold voltage is $1.0 \mathrm{~V}$. The colour scale ranges from 0 to 0.5 . 


\section{FE row

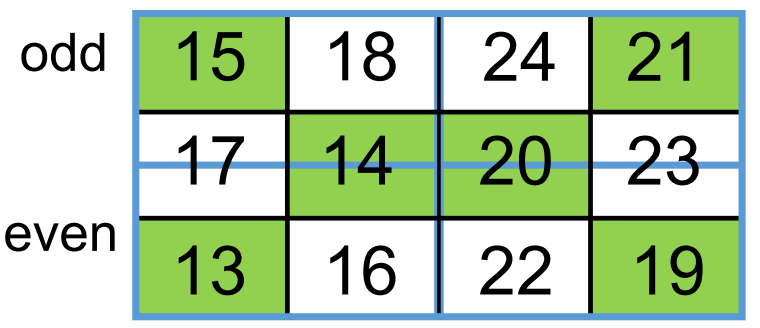 \\ even odd FE col}

Figure 6.32: Schematic drawing to illustrate the geometry layout in a region of two unit cells. The FE-I4 pixels are outlined in blue and the HV2FEI4 pixels are coloured in green and white.

as the threshold equivalent voltage (see Figure 6.18). Thus, the difference in the hit efficiency cannot be explained by these quantities. Furthermore, all parameter settings for the measurements with the three subpixel types are identical. Thus, the difference in the efficiency seems to be inherent in the design of the chip.

To study the in-pixel efficiency of the subpixel types, the subpixels were categorised into several geometries. Figure 6.32 illustrates the location of the 12 geometries in an area of two unit cells. The FE-I4 pixels are outlined in blue and the HV2FEI4 pixels are coloured in green and white. All sensor pixels that are in the bottom left corner of a readout chip pixel with even column and row number are shifted to the position of geometry 13 . The other pixels are shifted accordingly.

The geometries 1-12 are for the type A pixels, which are not discussed here, as well as all edge pixels of the HV2FEI4 sensor. The geometries 13-24 belong to the type A2 pixels. Their in-pixel efficiency maps are shown in Figure 6.33 with the same geometry numbers as in Figure 6.32. Figures 6.33a - 6.33d show that the pixels of geometries 15, 18, 24, and 21 have the highest efficiency as it is expected from the hit efficiency distributions. The centre parts of the subpixels are more efficient than the edges due to charge sharing. In Figure $6.33 \mathrm{e}-6.33 \mathrm{~h}$ a small misalignment is visible, because the area with the most efficient bins are not in the centre as it would expect if the layout of all subpixel types is the same. No difference between the pixels in one row is noted. Thus, pixels that belong to the same subpixel type behave alike.

In summary, the comparison of the three subpixel types showed, that no systematic difference in the ToT and $L v l 1$ distributions is evident for subpixels with the same configuration parameters. Only the hit efficiency differs for the three subpixel types due to a chip feature. 

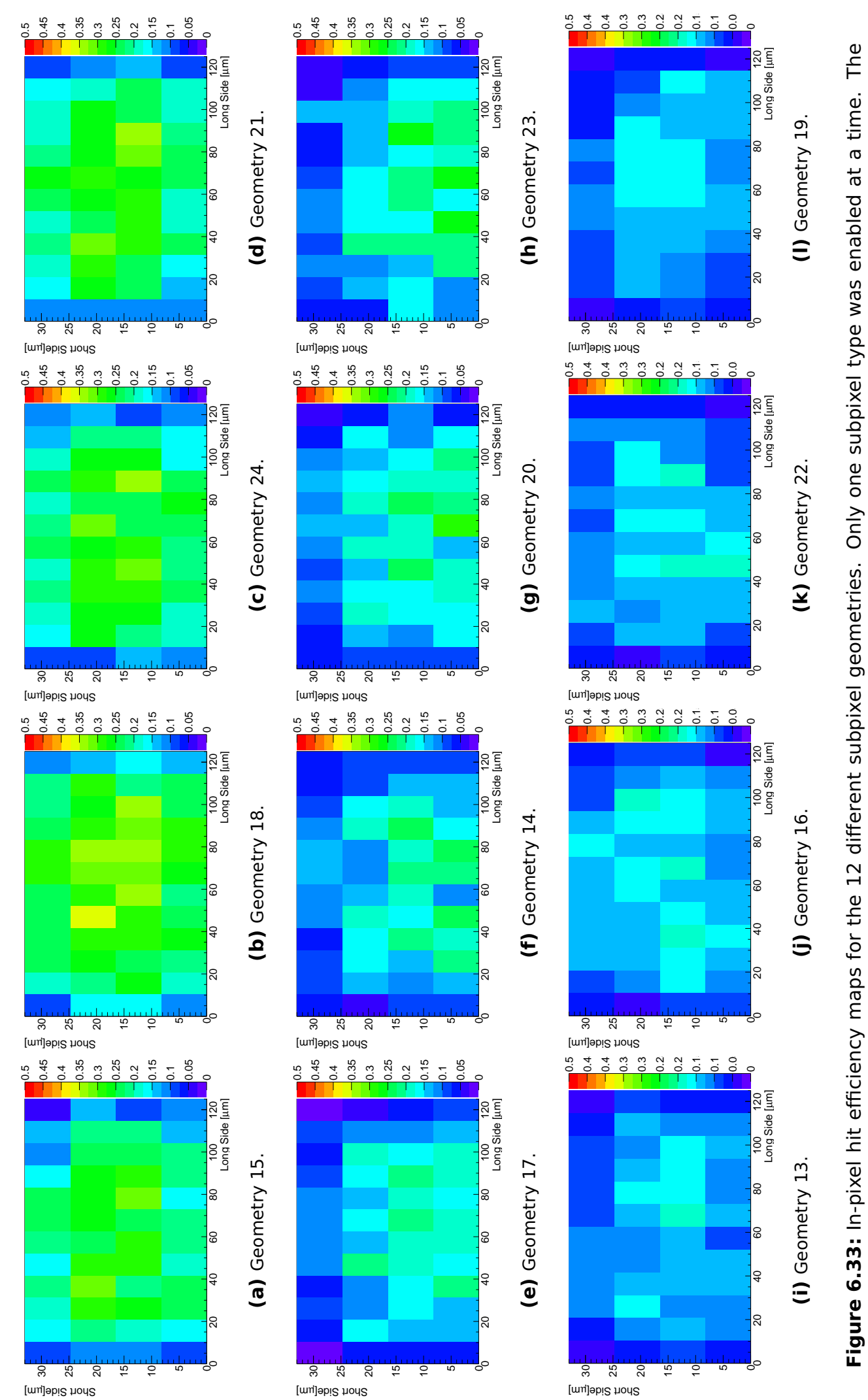


\subsubsection{Studies with Subpixel Type 1}

To investigate the CCPD module in more detail, test beam measurements were performed with only subpixel type 1 enabled as a representative for all subpixel types. The effect of different parameter settings on the module performance was studied. The parameter setting and conditions are the same as in the previous section unless stated otherwise.

A very broad $L v l 1$ distribution was observed in the previous section. For the usage in the ATLAS Experiment the width of the $L v l 1$ distribution needs to be reduced to one bin, because only one bin is read out. The influence of the feedback parameter VNFB on the Lvl1 distribution is shown in Figure 6.34 for two external threshold voltages. It can be seen that the width of the $L v l 1$ distribution decreases with increasing VNFB value. This effect is less pronounced for a smaller external threshold voltage. A similar behaviour was observed in the influence of the VNFB setting on the threshold equivalent voltage (see Figure 6.17). The influence is larger for a high external threshold voltage. Consequently, the VNFB setting has an influence on the width of the Lvl1 distribution.

Furthermore, the $L v l 1$ distribution for several external threshold voltages is presented in Figure 6.35 for $V N F B=1$. The peak position of the $L v l 1$ distribution is shifted to lower $L v l 1$ values with decreasing Th. Furthermore, the width of the distribution decreases with the external threshold voltage. Hence, the external threshold voltage has also an influence on the $L v l 1$ distribution. It cannot be identified, which quantity has the larger impact on the Lvl1 distribution, but the width and the peak position are influenced by a combination of the VNFB setting and the external threshold voltage.

The hit efficiency of the CCPD module is one of the key quantities in the suitability studies for the ITk. The influence of the external threshold voltage and the sensor bias voltage on the hit efficiency was investigated. Figure 6.36 shows the hit efficiency as a function of the external threshold voltage at a sensor bias voltage of -50 V. Again, the measurements indicate, that the threshold charge of the comparator is high compared to the expected MIP signal. It can be seen, that the efficiency increases with decreasing $T h$, as expected. The standard deviation of the hit efficiency distribution, which is displayed as error bar, also decreases. The mean hit efficiency at the minimal stable external threshold voltage of $0.89 \mathrm{~V}$ is 0.79 and the upper limit of the hit efficiency distribution is 0.95 (see Appendix Figure A.6e). Consequently, with the optimal parameter settings a high hit efficiency is achievable.

Furthermore, the hit efficiency was measured for sensor bias voltages between $-10 \mathrm{~V}$ and $-60 \mathrm{~V}$. All hit efficiency versus Th graphs and the hit efficiency distributions can be found in the Appendix in Figure A.5 and A.6. Similar results as for a sensor bias voltage of -50 V are observed with some fluctuations. To summarise these measurements, Figure 6.37 shows the hit efficiency as a function of the sensor bias voltage for an external threshold voltage of $0.89 \mathrm{~V}$. No systematic increase of the hit efficiency as a function of the sensor bias voltage is evident, as it was expected. The plots for the other external threshold voltages are in the Appendix in Figure A.7.

The sensor bias voltage has no significant influence on the hit efficiency. A possible explanation is that the width of the depletion zone is not significantly increasing. Consequently, 


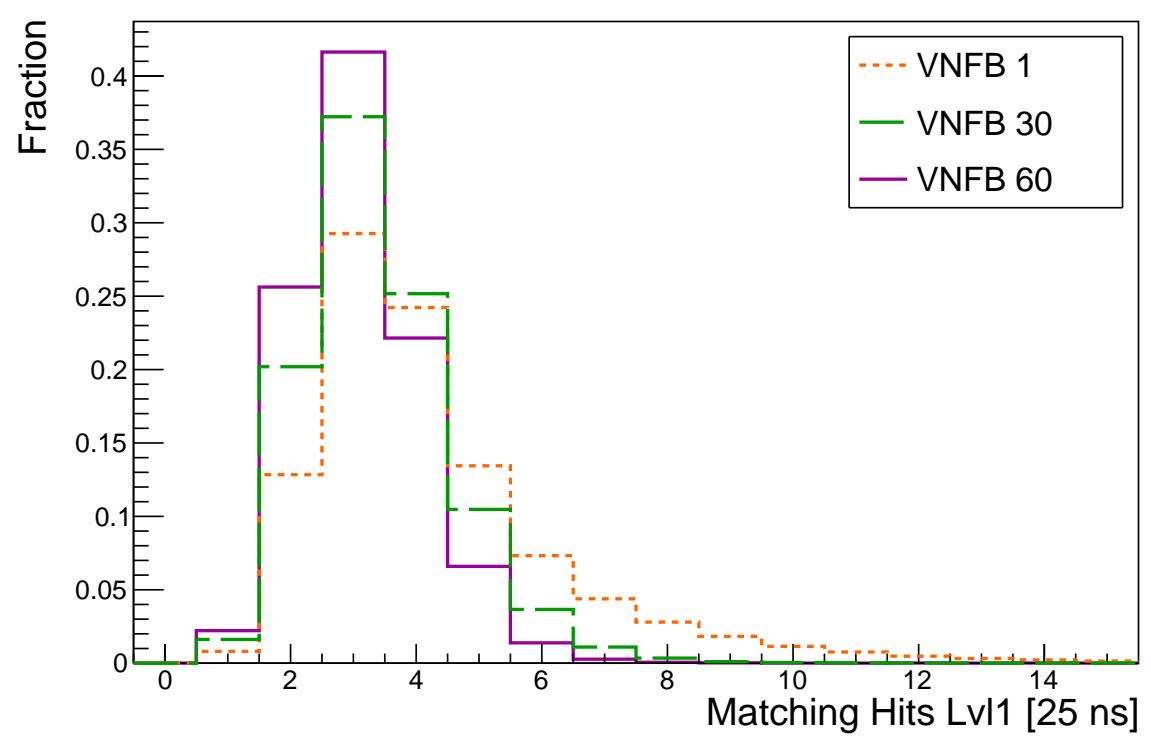

(a) $\mathrm{Th}=0.89 \mathrm{~V}$.

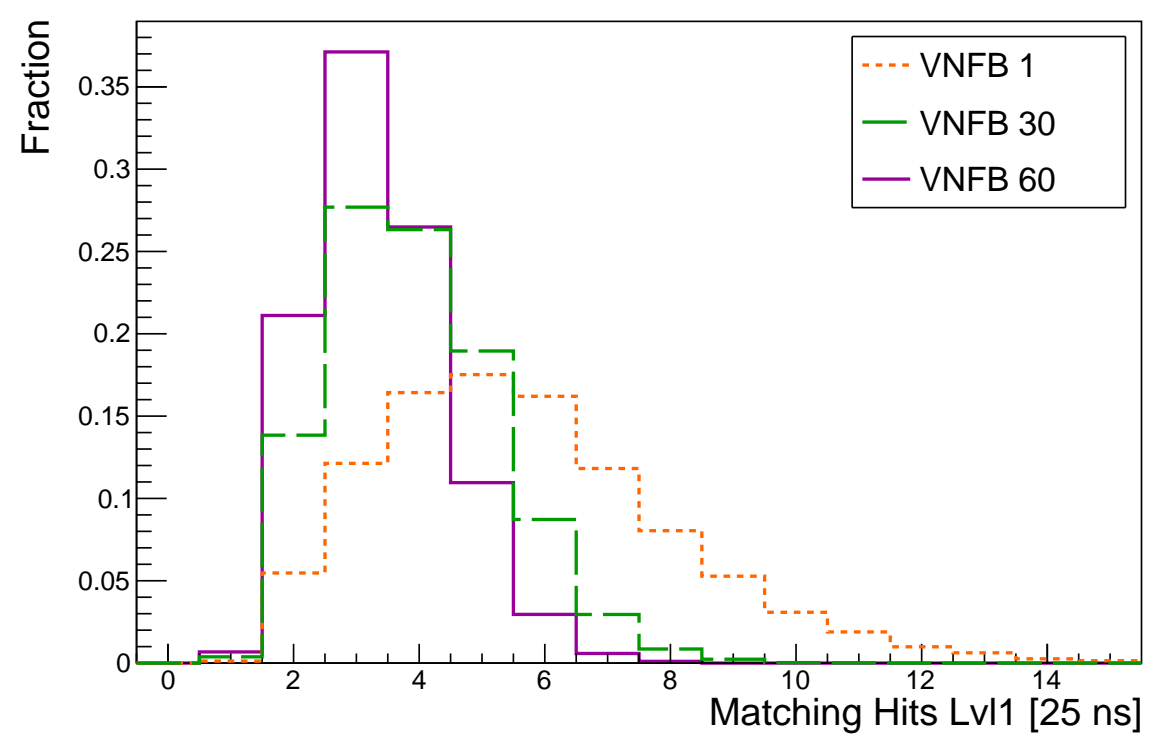

(b) $\mathrm{Th}=1.00 \mathrm{~V}$.

Figure 6.34: $L v / 1$ distributions for three different feedback settings with one subpixel type 1 enabled and for two external threshold voltages.

an increase in the hit efficiency would not be noticed in the broad hit efficiency distribution. Another explanation is that the charge is not only collected by drift, but also by diffusion, which is consistent with the spread of the $L v l 1$ distribution. Figure 6.38 shows the $L v l 1$ distributions for different sensor bias voltages at an external threshold voltage of $0.89 \mathrm{~V}$. The peak of the $L v l 1$ distribution is shifted to smaller $L v l 1$ values for a decreasing 


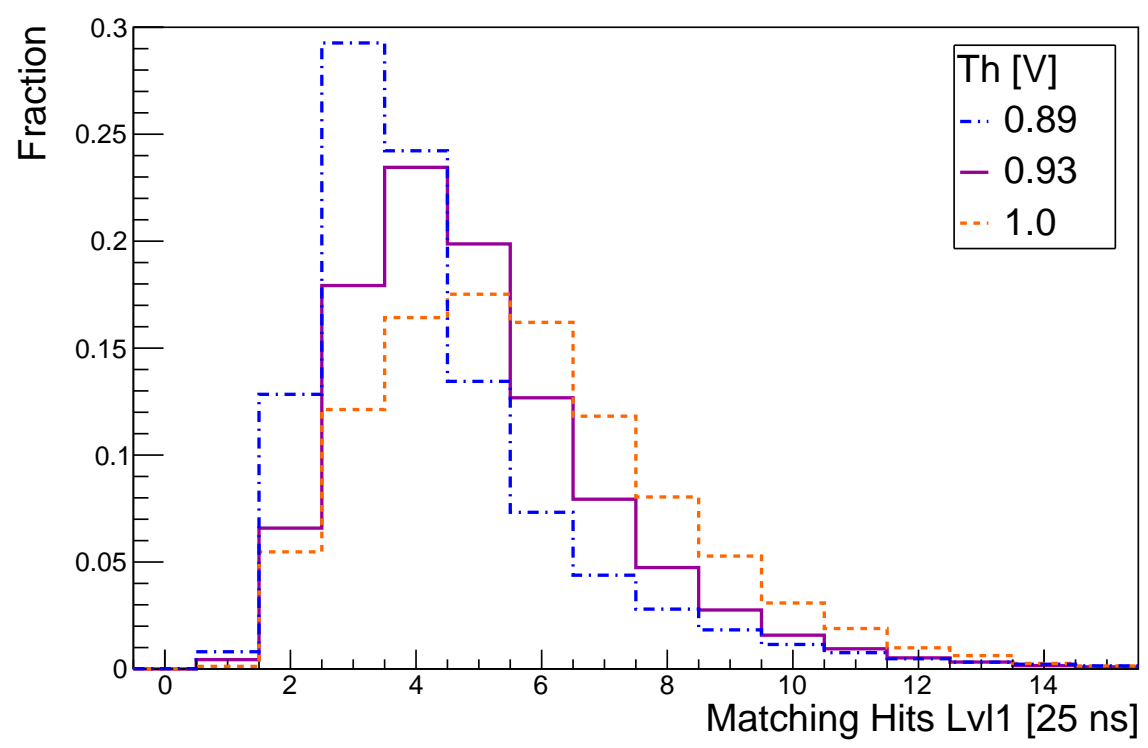

Figure 6.35: $L v l 1$ distributions for several external threshold voltages with one subpixel type 1 enabled at a feedback setting of $V N F B=1$.

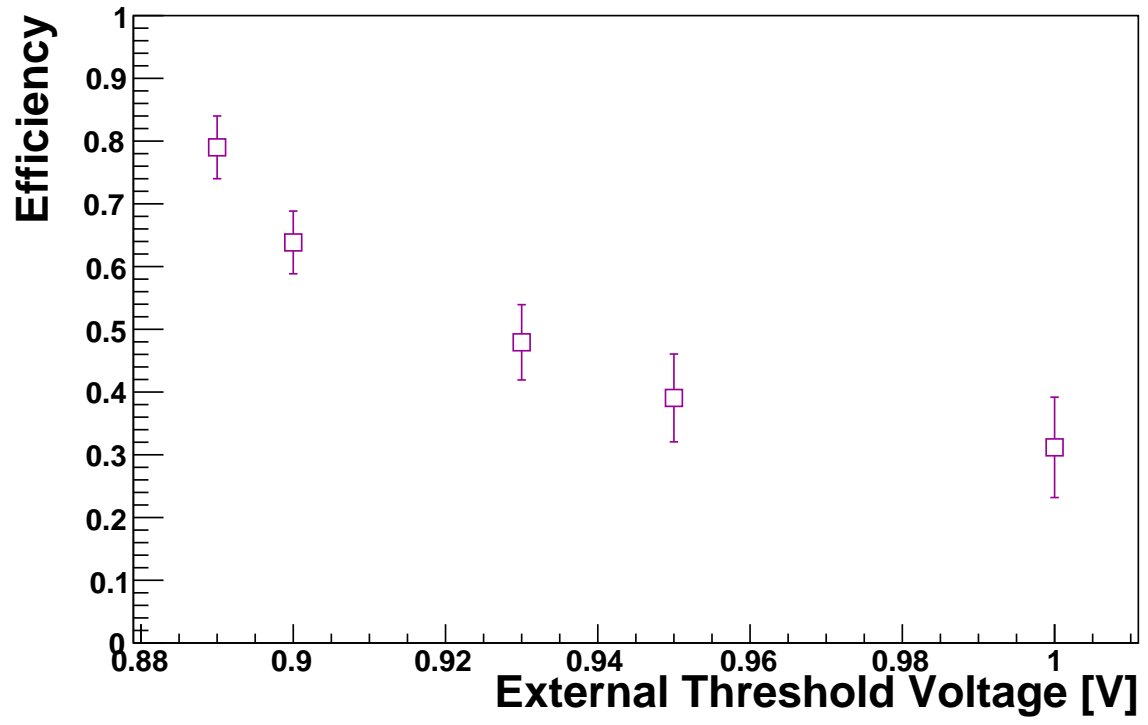

Figure 6.36: Hit efficiency as a function of the external threshold voltage at a sensor bias voltage of $-50 \mathrm{~V}$. Only subpixel type 1 is enabled. The error bar indicates the standard deviation of the efficiency distribution. 


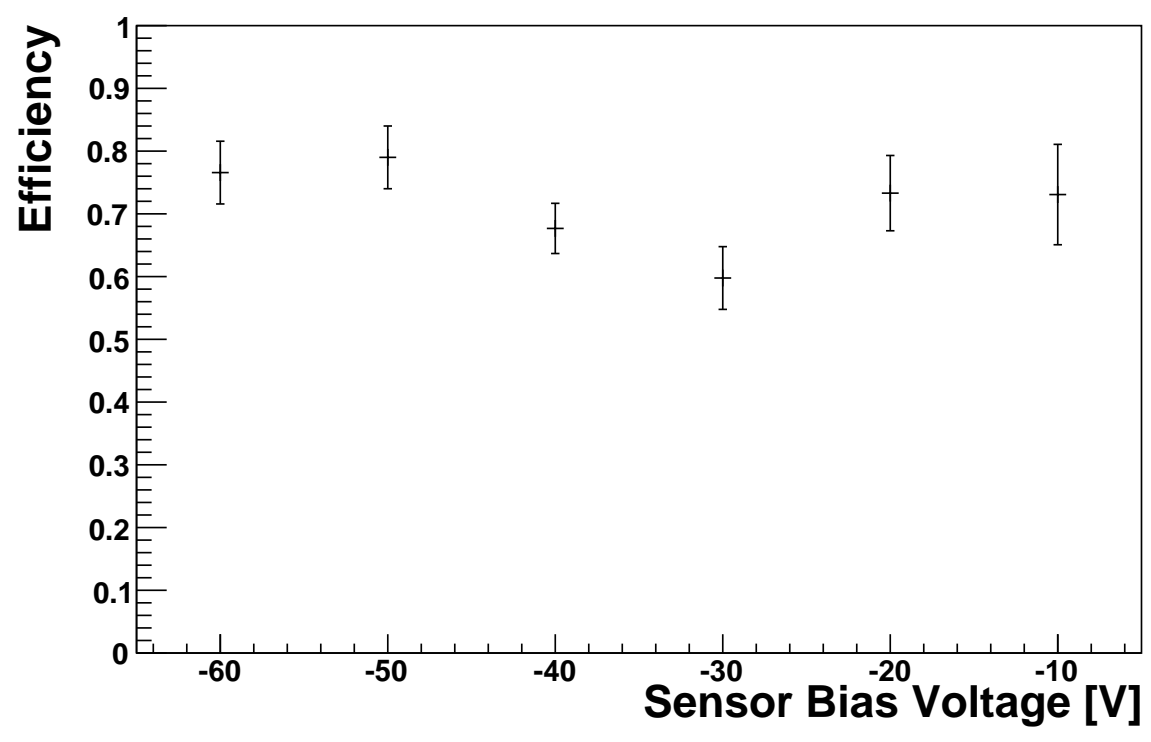

Figure 6.37: Hit efficiency as a function of the sensor bias voltage for an external threshold voltage of $0.89 \mathrm{~V}$. Only subpixel type 1 is enabled. The error bar indicates the standard deviation of the efficiency distribution.

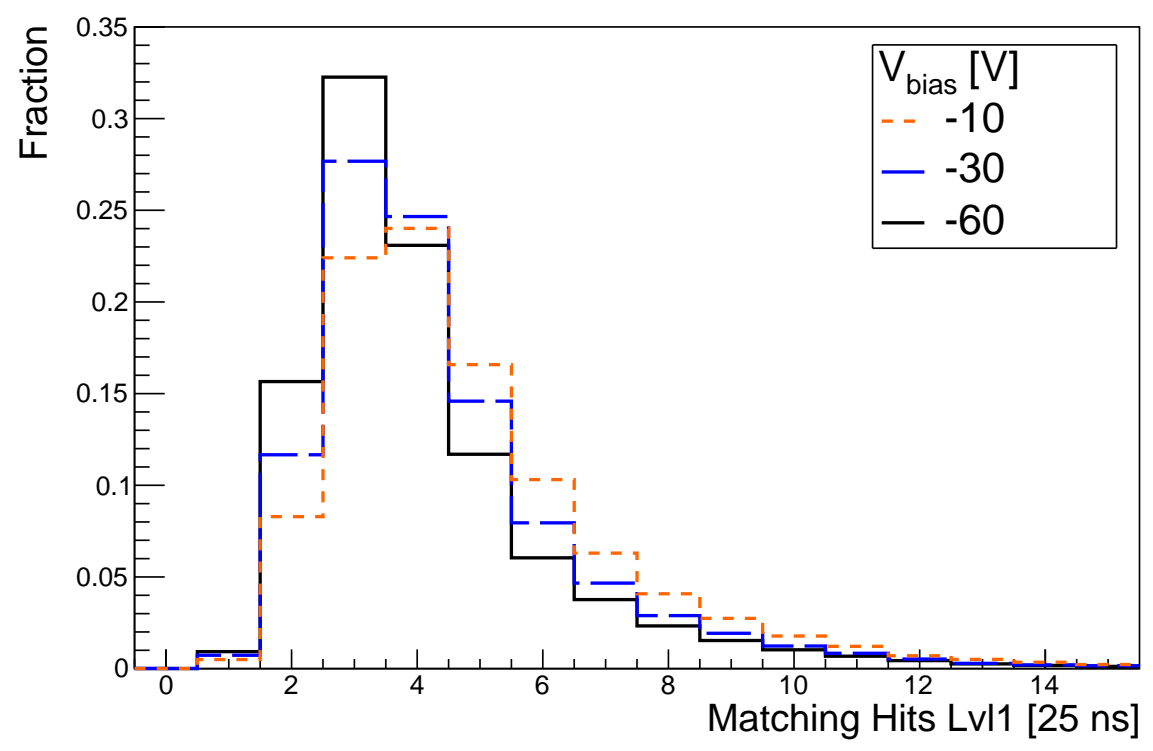

Figure 6.38: $L v / 1$ distributions for several sensor bias voltages at an external threshold of $0.89 \mathrm{~V}$ with one subpixel type 1 enabled. 
sensor bias voltage. Consequently, the charge is collected faster for a lower sensor bias voltage. The charge fraction that is collected via drift increases and the diffusion component decreases. The total effect is explained by a mixture of charge collection by drift and by diffusion.

\subsection{Subpixel Decoding}

\subsubsection{Subpixel Position}

Before any further characterisation, the positions of the respective subpixels in the HV2FEI4 coordinate system have to be verified. It is known, that the parameter VNOut1, VNOut2 and VNOut3 define the output signal amplitude of the three subpixels. Furthermore, the chip description points out that every second column of the HV2FEI4v2 is mirrored along the small pixel side. But it is neither known, which of the six subpixels in a unit cell is connected to which FE-I4 pixels, nor which VNOut1/2/3 parameter controls the output signal height of which subpixel type.

To figure this out, some special runs were taken in test beam measurements at DESY. At first, only one subpixel type was enabled per run. During test beam data reconstruction, all runs were aligned with a fixed set of alignment constants, even for runs with different subpixels. Consequently, the reconstructed tracks get a common coordinate system. The interpolated tracks are all plotted in this coordinate system. Figures 6.39 and 6.40 show the projection of the interpolated hit positions from good tracks (see definition in Section 5.3.6) that caused a hit in the reference module for the $x$-and $y$-direction. For the

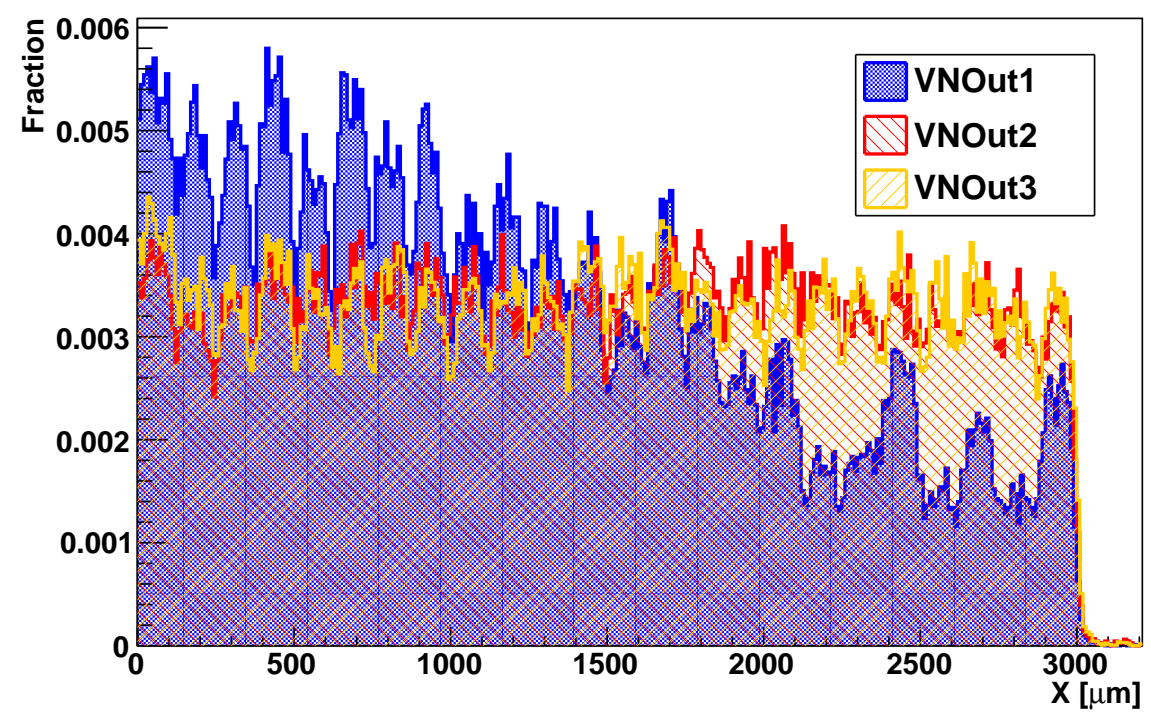

Figure 6.39: Projection of interpolated hit positions from good tracks in $x$-direction that caused a hit in the reference module. Only one subpixel type is enabled at a time. 


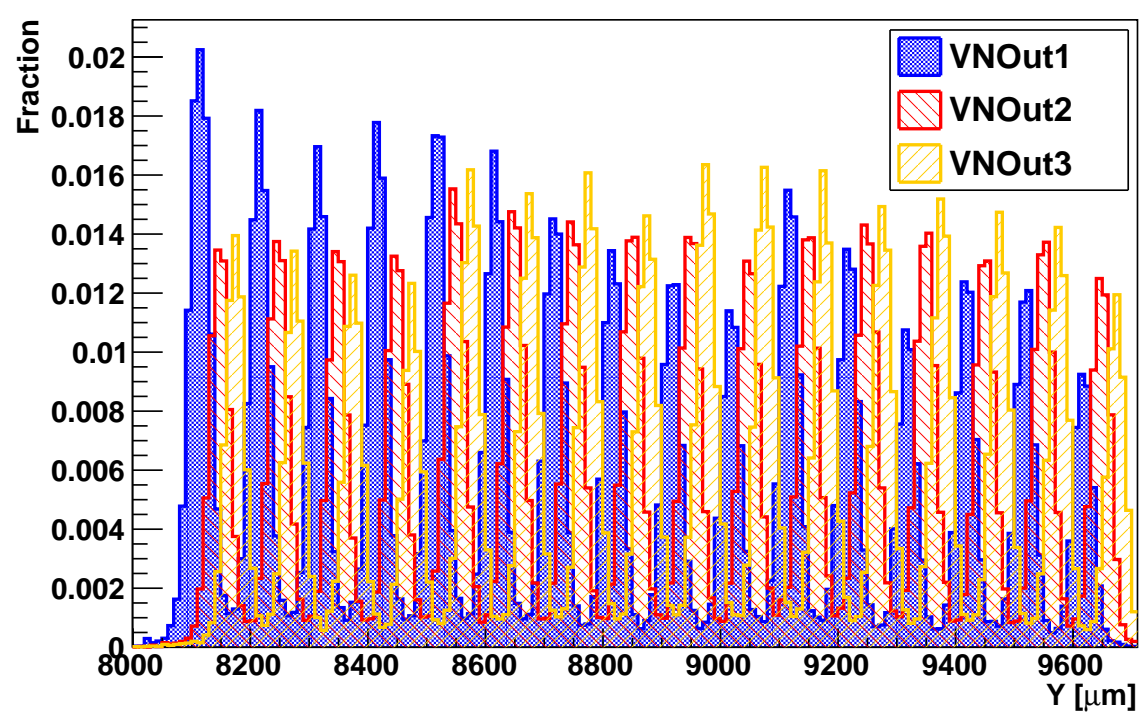

Figure 6.40: Projection of interpolated hit positions from good tracks in $y$-direction that caused a hit in the reference module. Only one subpixel type is enabled at a time.

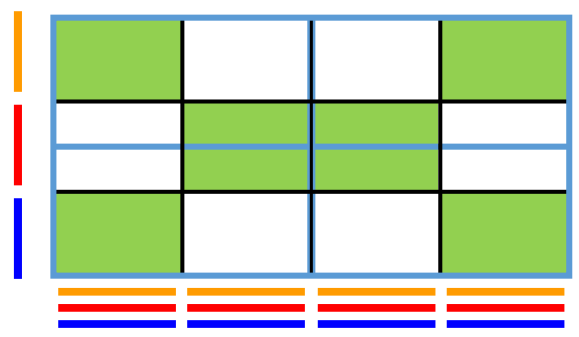

Figure 6.41: Schematic drawing of two unit cell areas illustrating the conclusions, which were drawn from Figure 6.39 and 6.40.

$x$-direction no conclusion can be drawn, because all $x$-positions show histogram entries in Figure 6.39. The decreasing number of tracks with increasing $x$-position for VNOut 1 will be addressed later.

In the $y$-direction the subpixel structure is visible. The histogram 6.40 has only entries in certain positions for the different VNOut DACs. Consequently, the column number $0,3,6, \ldots$ are controlled by VNOut $1,1,4,7, \ldots$ by VNOut 2 and $2,5,8, \ldots$ by VNOut 3 . These column numbers are the HV2FEI4v2 real column numbers as defined in Figure 6.1.

This information on the subpixel position is illustrated in Figure 6.41. It shows the area of two unit cells with 12 HV2FEI4v2 pixels (green and white) and four FE-I4 pixels (blue). The colours at the edges indicate the possibility that this column/row is controlled by VNOut1 (blue), VNOut2 (red) or VNOut3 (orange). The colours are the same as in Figures 6.39 and 6.40 . 


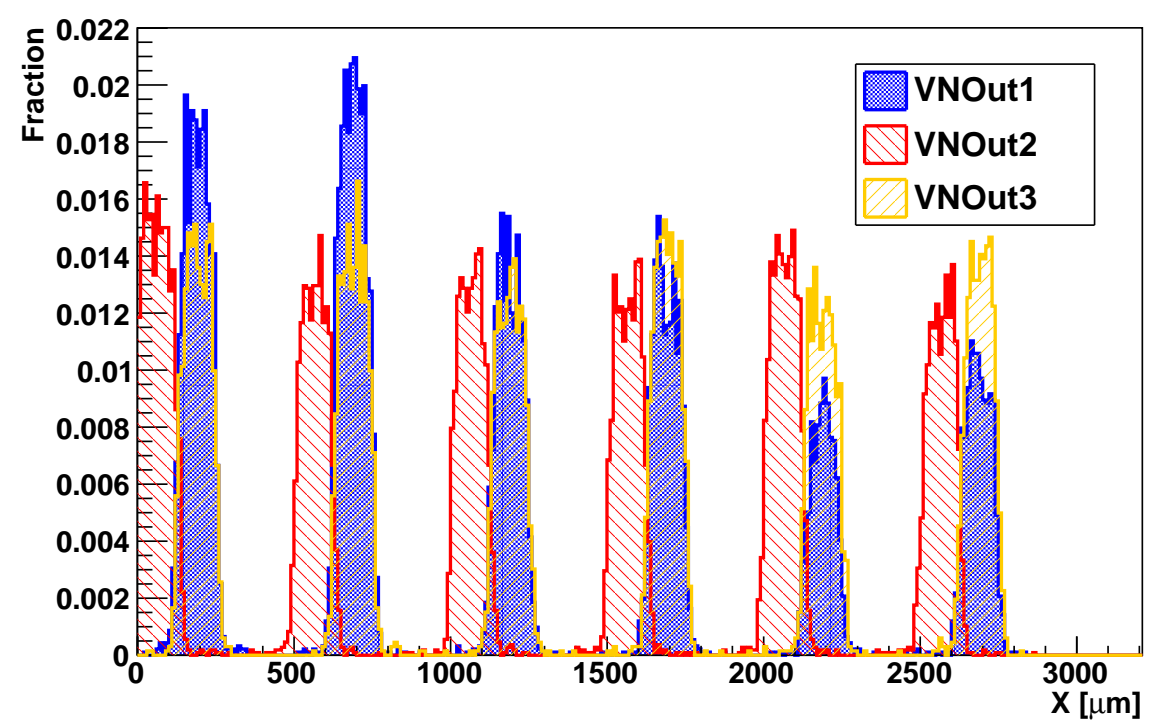

Figure 6.42: Projection of interpolated hit positions from good tracks in $x$-direction that caused a hit in reference module. Only one subpixel type and every second FE-14 column and row are enabled at a time.

To get more information about the subpixel position and the FE-I4 pixel, to which they are connected, measurements were performed enabling only every second FE-I4 column and row.

Figure 6.42 shows the projection of the interpolated hit positions in $x$-direction with this FE-I4 configuration. The projected hit positions in $x$-direction for subpixel type 2 (red) are clearly distinguishable from the projected hit positions of subpixel types 1 and 3 (blue and yellow), which overlap. It can be seen that Figures 6.43 for the $y$-direction looks similar to Figure 6.40. These results allow the identification of the position of each subpixel type in the HV2FEI4v2 pixel matrix, as well as the FE-I4 pixel they are connected to. As illustrated in Figure 6.44, the marked FE-I4 pixel is connected to the three labelled subpixels. The colours at the edges indicate that this column/row is controlled by the VNOut1/2/3 parameter.

\subsubsection{Subpixel Mapping}

With the knowledge of the subpixel positions, the characteristics of the three subpixel types can be investigated. With the ToT tuning, as it is described in Section 6.2.4, test beam measurements were performed at DESY in November 2015. Only one subpixel type was enabled per measurement run.

The normalised ToT distributions of the ToT sum of all hits in a cluster with a matched track for the three subpixel types are shown in Figure 6.45. The subpixel type 1 has the narrowest distribution compared with the other subpixels. The reason is that the FDACs are tuned to a signal amplitude as generated by the VNOut1 settings. In addition, the distribution for the subpixel type 1 has the smallest overlap with the other distributions, 


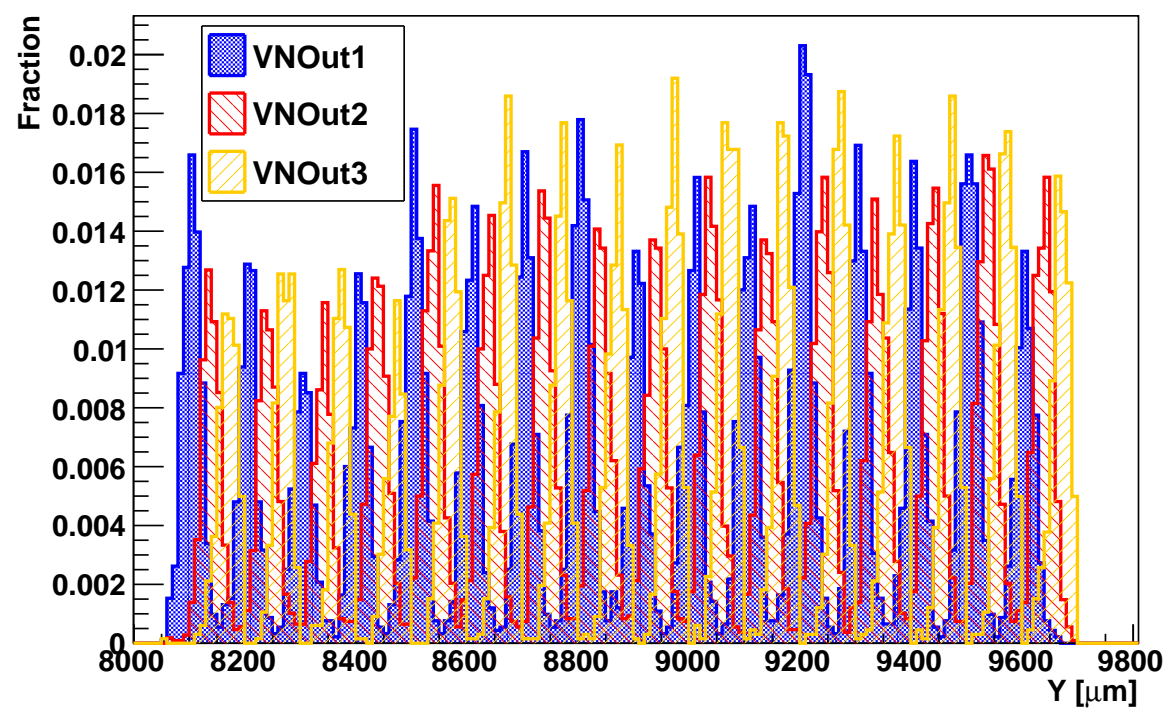

Figure 6.43: Projection of interpolated hit positions from good tracks in $y$-direction that caused a hit in reference module. Only one subpixel type and every second FE-I4 column and row are enabled at a time.

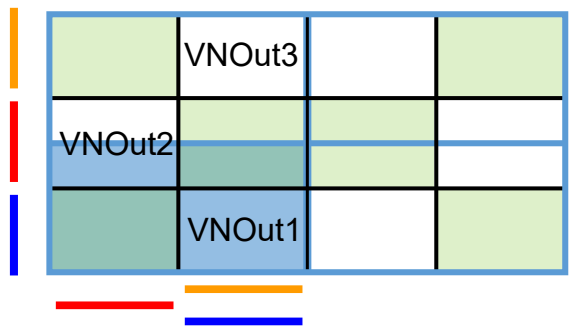

Figure 6.44: Schematic drawing of two unit cell areas to illustrate the position of each subpixel type in the HV2FEI4v2 pixel matrix, as well as the FE-14 pixel they are connected to.

whereas the distributions of subpixel types 2 and 3 have a larger overlap.

With these distributions cuts were defined to separate the three subpixels by their ToT response. In a measurement with all subpixel types enabled, the subpixels are mapped accordingly. If the ToT is below 6, the hits are assigned to the subpixel type 1 . If the ToT is 11 or larger, the hits are mapped to the subpixel type 2 and in between the hits are assigned to the subpixel type 3.

To get a larger separation power, other variables were studied. Figure 6.46 shows the Lvl1 distributions of the three subpixel types. The $L v l 1$ distributions are again very broad. A point to note is that the peaks of the $L v l 1$ distributions of the three subpixels differ by two bins from each other. The subpixel type 2, which has the largest ToT, has the smallest $L v l 1$ and the subpixel type 1 with the smallest ToT accumulated at the higher $L v l 1$. This 


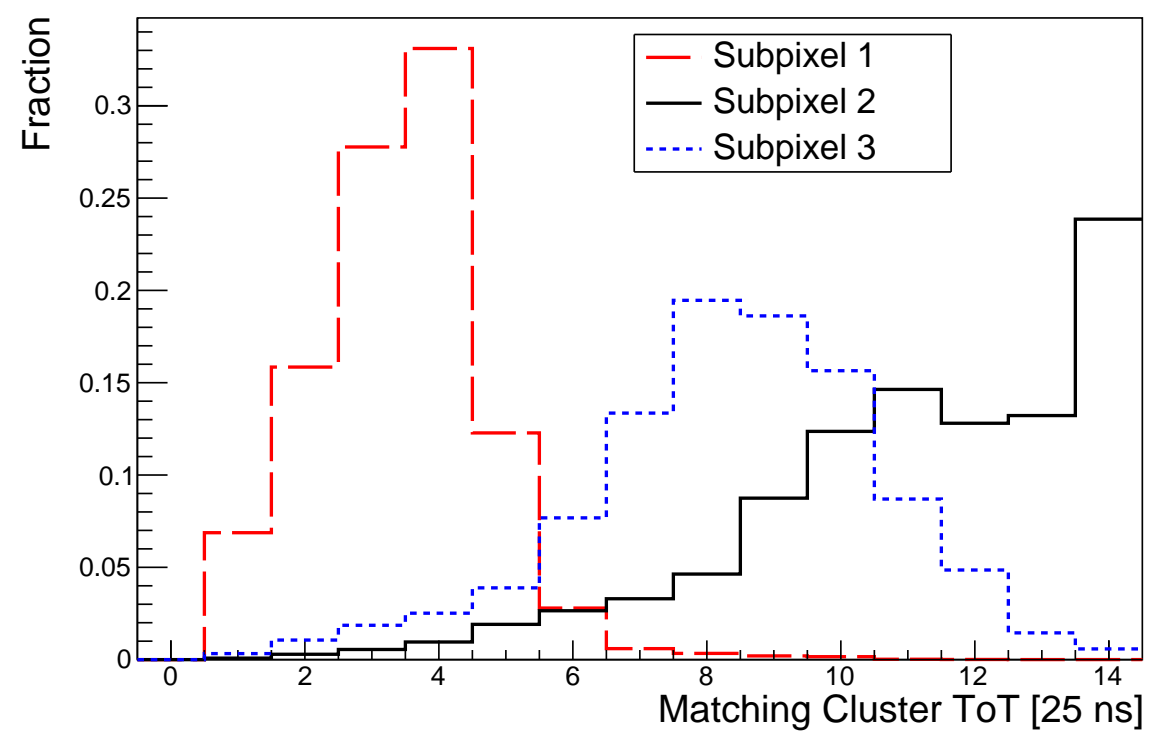

Figure 6.45: TOT distributions for three separate test beam measurements with only one enabled subpixel type at a time.

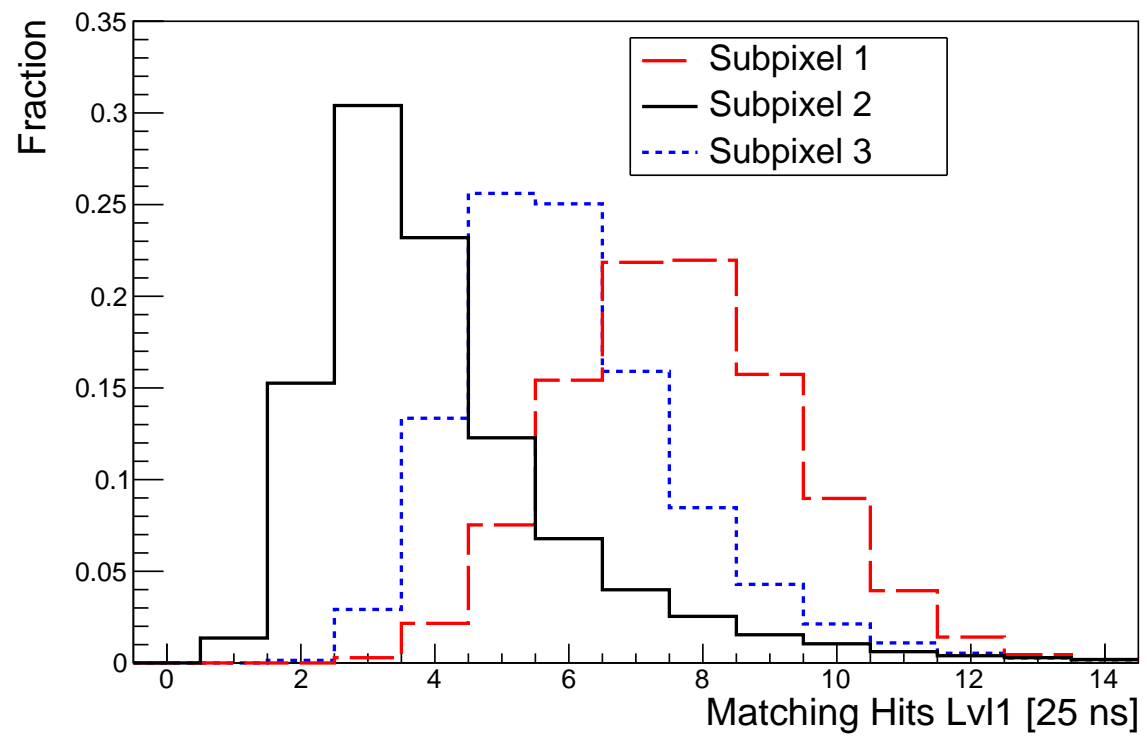

Figure 6.46: $L v l 1$ distributions from three separate test beam measurements with only one enabled subpixel type at a time. 
behaviour can be attributed to a combination of effects of time walk in the HV2FEI4 as well as the FE-I4.

For the analysis of the test beam measurements an additional separation power from the Lvl1 distributions is available to distinguish between the hits from different subpixel types.

To combine the two variables, ToT and $L v l 1$, their values for hits from the test beam measurement with one subpixel type enabled were entered into a 2-dimensional histogram. The three resulting histograms with the ToT and $L v l 1$ information are shown in Figure 6.47. The normalised number of entries, which is colour-coded in the histograms, can be interpreted as probability that this set of ToT and Lvl1 can be assigned to the subpixel type. Thus, every measured variable set

$$
\vec{x}=\left(\begin{array}{c}
T o T \\
L v l 1
\end{array}\right)
$$

is connected to a probability $p(\vec{x})$ for a certain parameter $\theta$, which are the three subpixel types.

These quantities allow the use of a likelihood function to map the subpixels. The likelihood function is used to estimate a parameter $\theta$ from a set of statistics. It gives the joint probability to obtain a data sample consisting of $n$ independent measurements $x_{1}, \ldots, x_{n}$

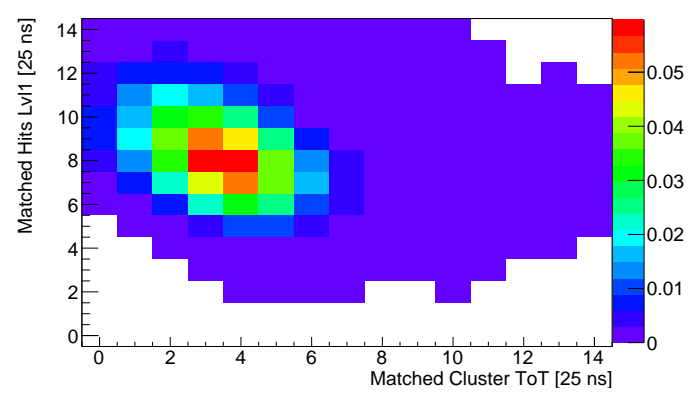

(a) Subpixel 1.

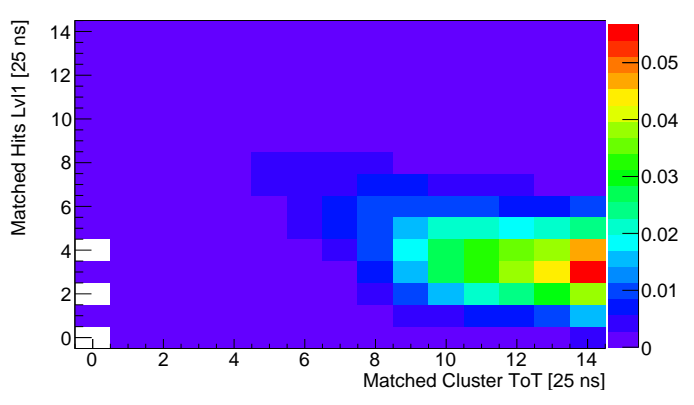

(b) Subpixel 2.

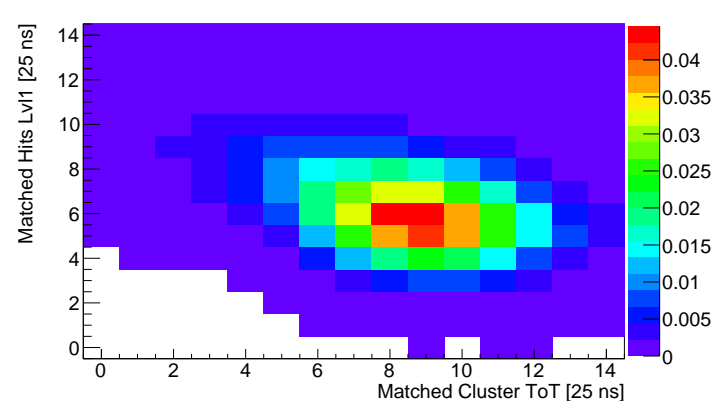

(c) Subpixel 3.

Figure 6.47: $T O T$ and $L v / 1$ distributions from three separate test beam measurements with only one enabled subpixel type at a time. 
given a model with some parameter $\theta$ as

$$
\mathscr{L}(\theta)=p\left(x_{1}, \ldots, x_{n} \mid \theta\right) .
$$

In the test beam data reconstruction, the $T o T$ and $L v l 1$ values are registered for a hit in a FE-I4 pixel. The likelihood values for the three histograms (Figure 6.47) are read in and the subpixel type with the highest value is mapped to the hit.

\subsubsection{Comparison of Cut and Likelihood Method for Subpixel Mapping}

In order to study the differences of the methods for the subpixel mapping, the cut and the likelihood method, the residual and hit efficiency plots were compared. The test beam measurements were performed with the standard parameters (see Table 6.1 and 6.2) and a sensor bias voltage of $-40 \mathrm{~V}$. Only good tracks, as defined in Section 5.3.6, were considered for the analyses. Furthermore, only the type A2 sensor pixels are included in the results.

For the residual distributions in $x$ - and $y$-direction only tracks that result in a hit in exactly one pixel are considered. Consequently, the width of the residual distribution cannot be larger than $125 \mu \mathrm{m}$ in the $x$-direction and $33 \mu \mathrm{m}$ in the $y$-direction, which corresponds to the pixel size. Figure 6.48 shows the residual distributions in $x$-and $y$-direction for the cut method and Figure 6.49 for the likelihood method. As described in Section 6.3.1, the expected standard deviation values are $37.5 \mu \mathrm{m}$ and $13.8 \mu \mathrm{m}$ for the $x$-and $y$-direction, respectively. The standard deviations $\sigma$ of the residuals in Figures 6.48 and 6.49 are given in Table 6.5 for both methods. The measured standard deviations are smaller for

\begin{tabular}{l|c|c} 
& $\sigma_{x}[\mu \mathrm{m}]$ & $\sigma_{y}[\mu \mathrm{m}]$ \\
\hline Cut Method & 43.7 & 16.9 \\
\hline Likelihood Method & 40.9 & 15.3 \\
\hline Expected & 37.5 & 13.8
\end{tabular}

Table 6.5: Standard deviations of the $x$ - and $y$-residual distributions for the cut and the likelihood method as well as the expected values.

the likelihood method compared to the cut method. The obtained values for both methods are larger than expected. A reason for this effect is a mismatch of subpixel position. Because of subpixels that are mapped to the wrong position, the $x$-residual distributions have tails on both sides (see Figures 6.48a and 6.49a). The tails are less pronounced in the residual distribution from the likelihood method, which is a consequence of a better subpixel mapping compared to the cut method.

The number of mismatched hits was estimated from the tails of the $x$-residual distributions. Approximately $1.5 \cdot 10^{5}$ entries are in the tails of the distribution of Figure 6.48a obtained with the cut method compared with approximately $1.7 \cdot 10^{6}$ entries in the centre of the distribution. Therefore, approximately $9 \%$ of all hits are mapped to the wrong subpixel with the cut method. This number compares to approximately $5 \%$ incorrectly mapped subpixels using the likelihood method. The tails of the distribution of Figure 6.49a have approximately $10^{5}$ entries and the centre of the distribution has approximately $2.2 \cdot 10^{6}$ 


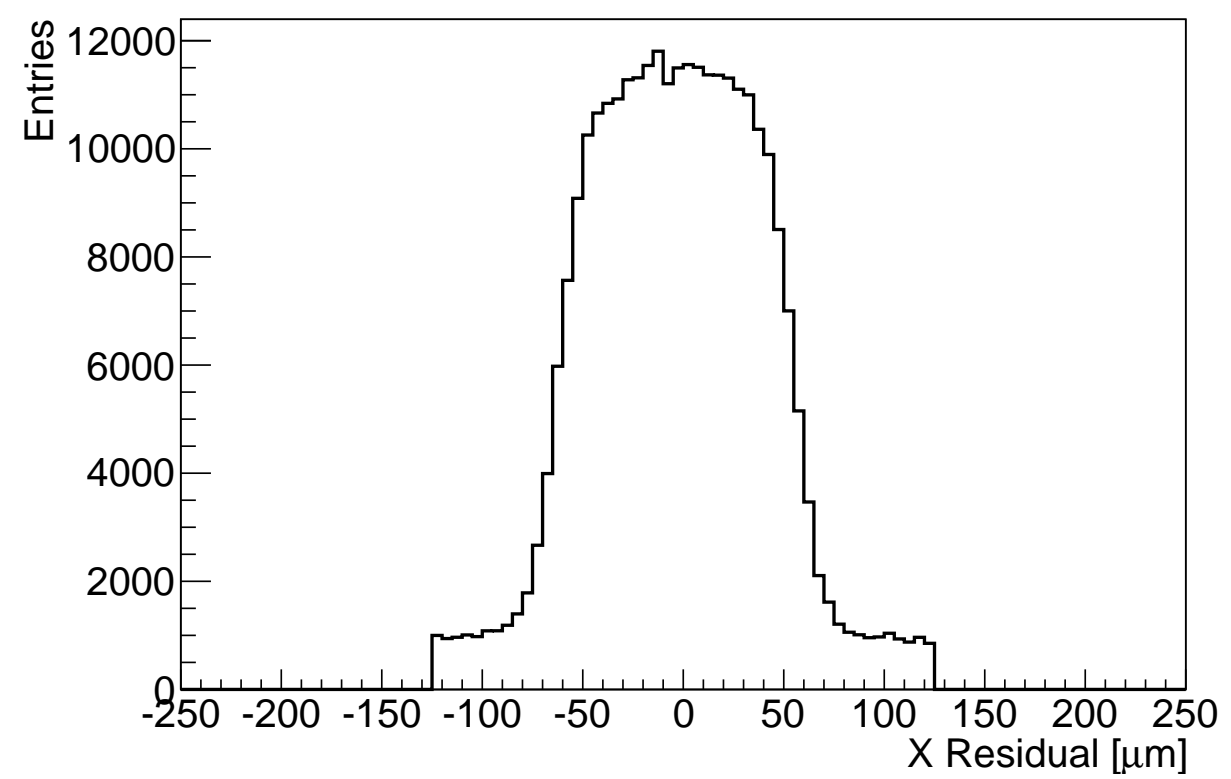

(a) $x$ residual - Cut Method.

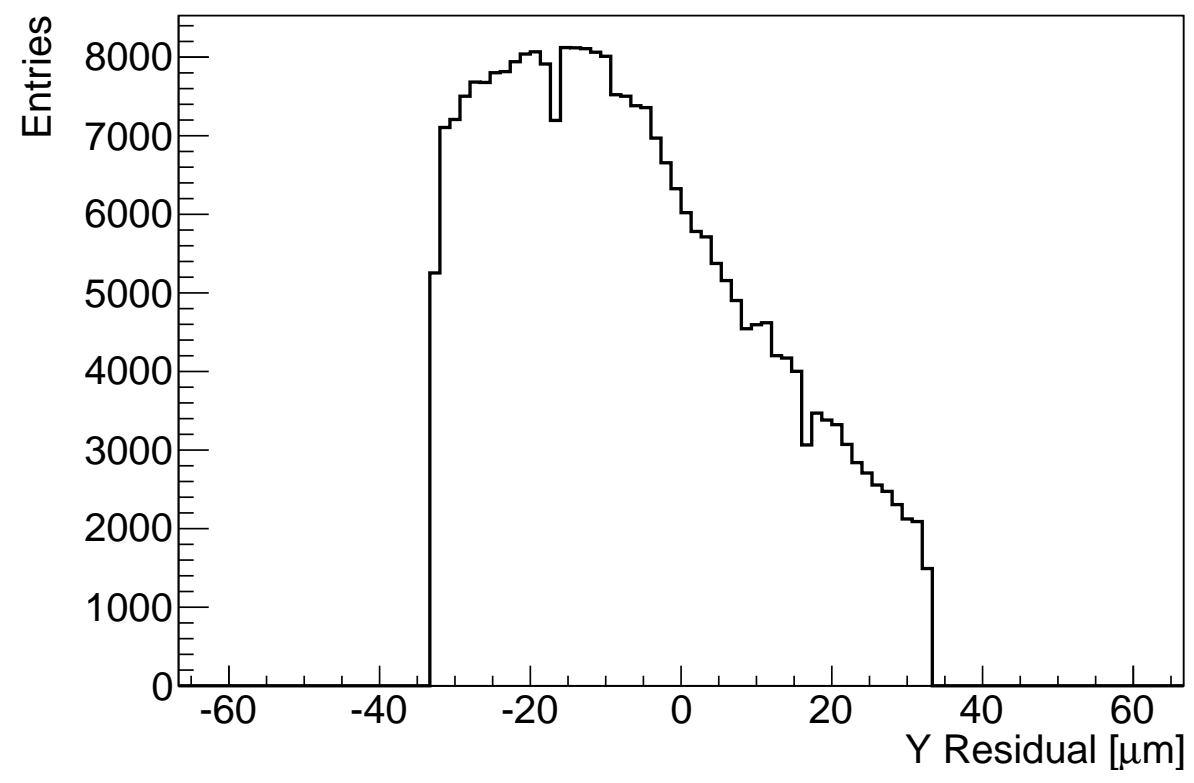

(b) y residual - Cut Method.

Figure 6.48: Residual distributions for $x$ - and $y$-direction with the cut method. 


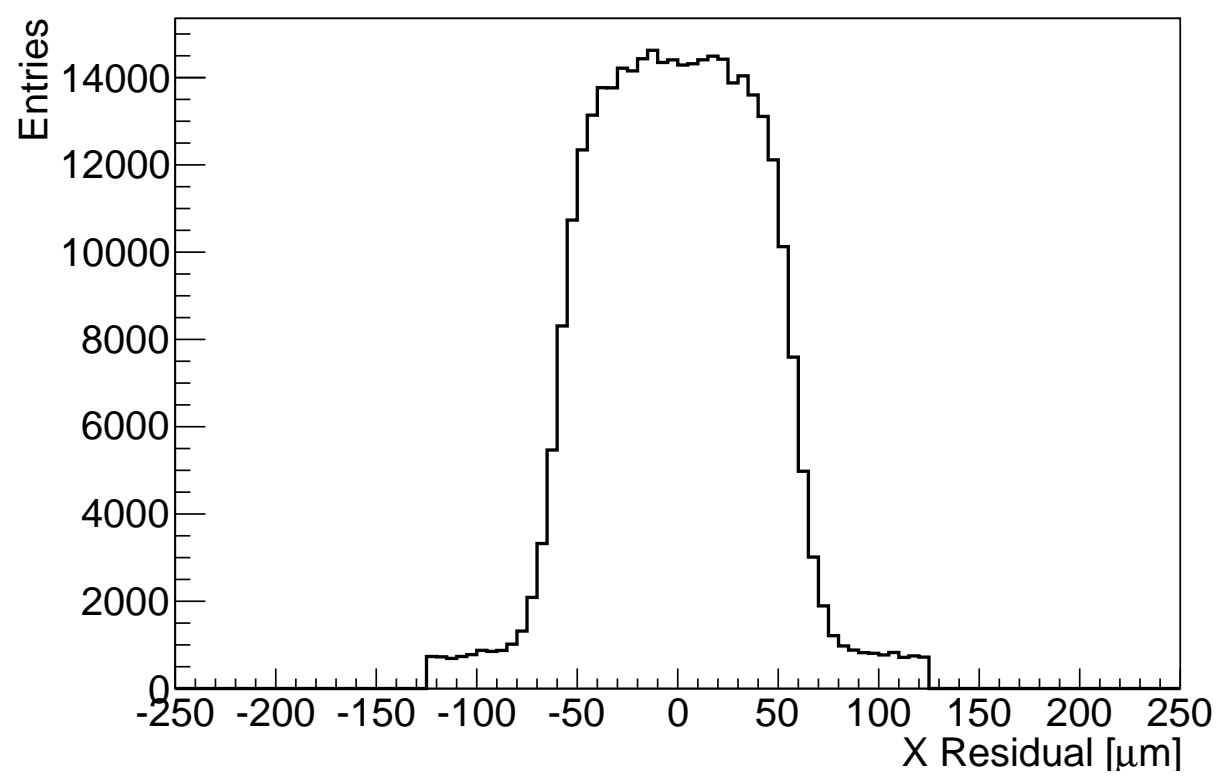

(a) $x$ residual - Likelihood Method.

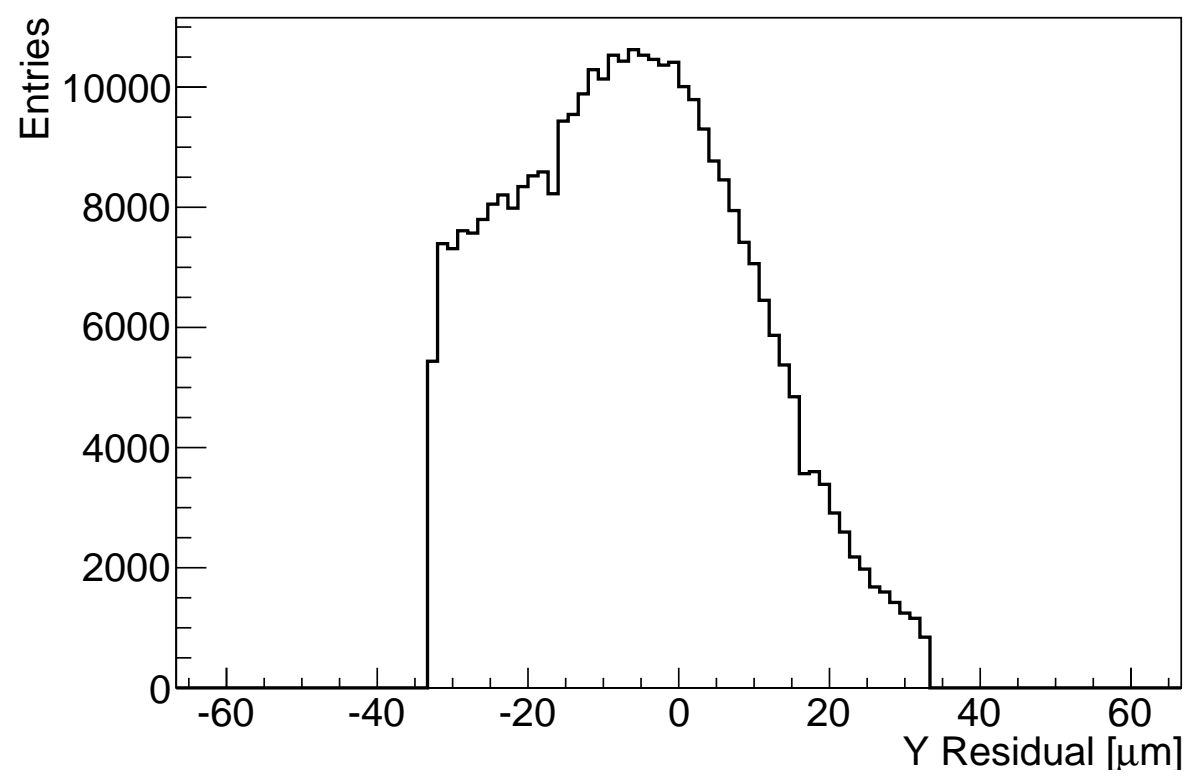

(b) y residual - Likelihood Method.

Figure 6.49: Residual distributions for $x$ - and $y$-direction with the likelihood method. 
entries.

The $y$-residuals are not symmetric around $0 \mu \mathrm{m}$ even if the mean value of the residual distribution from the likelihood method is closer to $0 \mu \mathrm{m}$ than the ones from the cut method. This effect can be explained by a misalignment of the tracks. The analyses with the cut and the likelihood method are independently aligned. The misalignment is more pronounced in the short pixel direction, because a small shift has a larger impact considering the total pixel size.

Furthermore, the subpixel mapping is influenced by particles that cause a hit in two subpixels that are connected to one FE-I4 pixel. In such a diagonal cluster the output signals of the two HV2FEI4 pixels is summed up, which would influence the subpixel mapping. The distribution of the cluster sizes is shown in Figure 6.50. No systematic difference between the cluster size distributions for the cut and the likelihood methods were observed. The number of clusters with one hit subpixel is 10 times larger than the number of clusters with two hit subpixels. The possibility of a diagonal cluster is even lower. Consequently, the effect of an incorrect subpixel mapping by a diagonal cluster is in the order of a few per cent.

The hit efficiency of the module is the next quantity, which was studied for the cut and the likelihood method. It is worth noting that the HV2FEI4v2 comparator threshold is at approximately $1000 e$ (see Figure 6.15) at the minimal stable external threshold voltage of $0.89 \mathrm{~V}$, which is comparable to the expected MIP signal. Consequently, the hit efficiency is not expected to be as high as possible.

Figure 6.51 shows the hit efficiency maps and distributions for the cut method and Figure 6.52 for the likelihood method. The regions without entries in the pixel map are caused by masked and noisy pixels. In the pixel maps (see Figures 6.51a and 6.52a) a difference

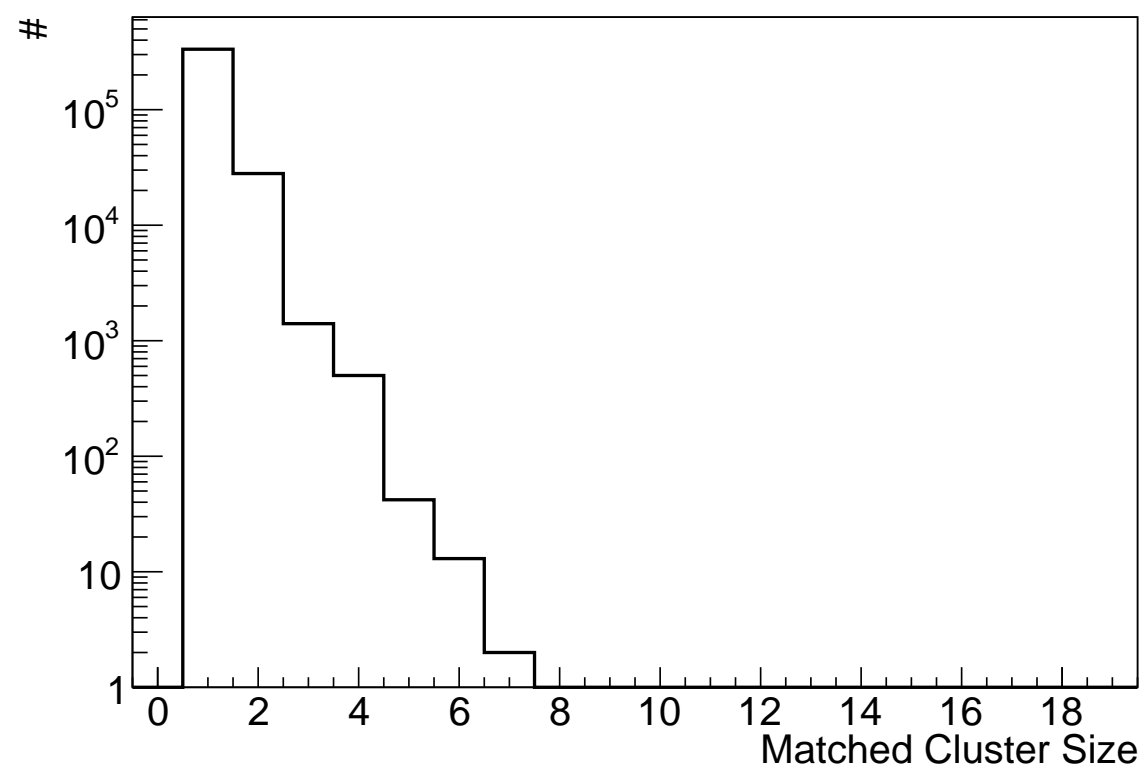

Figure 6.50: Distribution of cluster sizes with all three subpixel types enabled. 


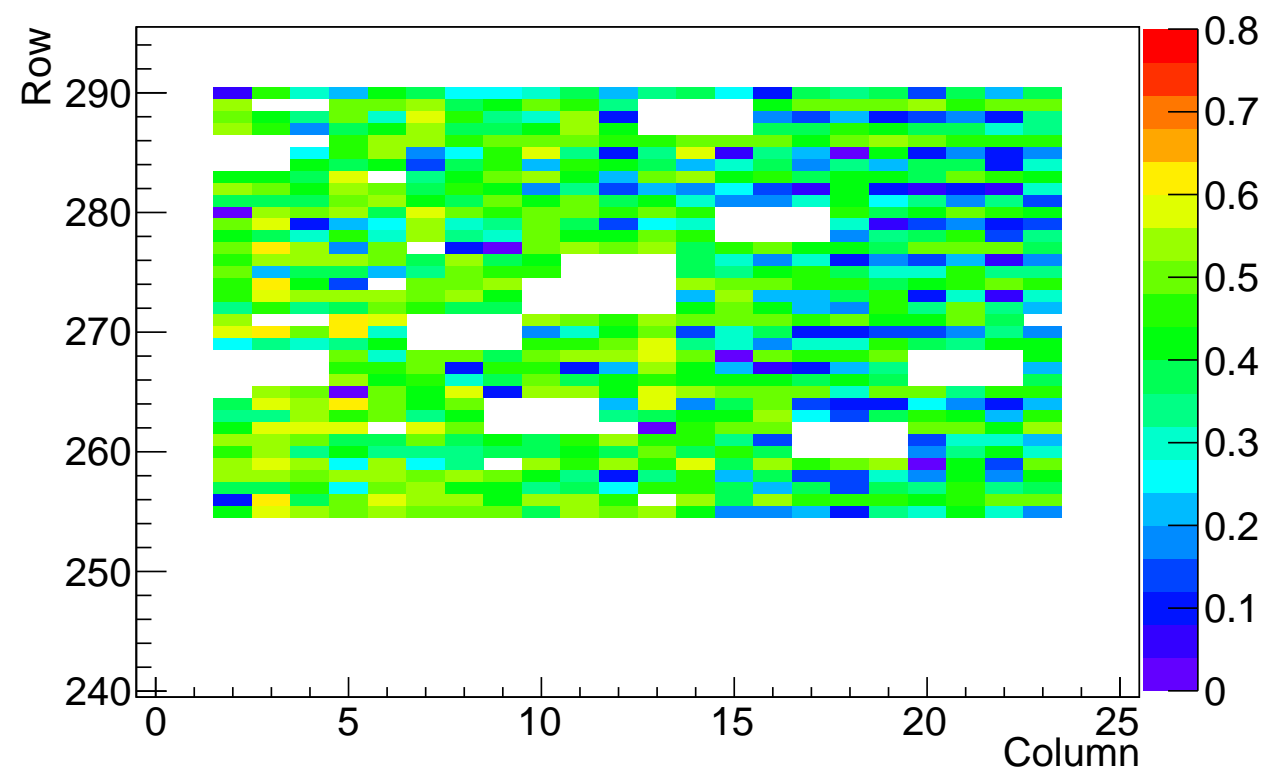

(a) Efficiency map - Cut Method.

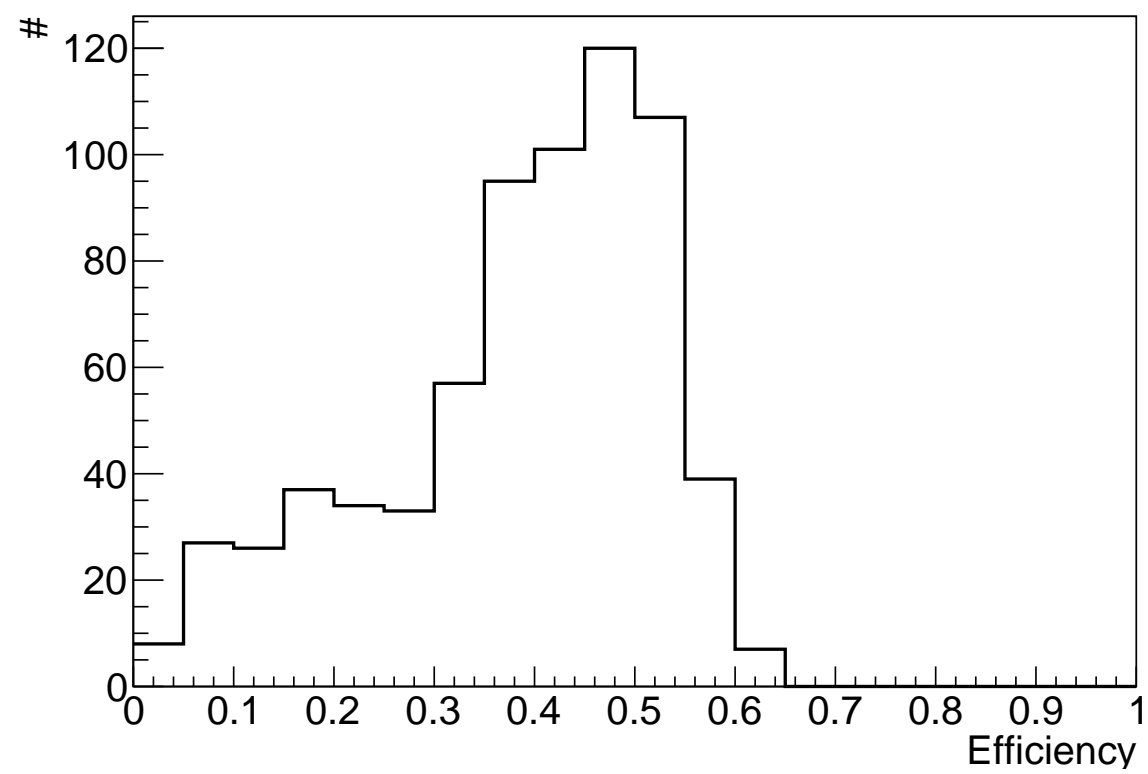

(b) Efficiency distribution - Cut Method.

Figure 6.51: Hit efficiency maps and distributions for the cut method. The pixel map shows the efficiency for every pixel. These values are histogramed in the distribution plots. 


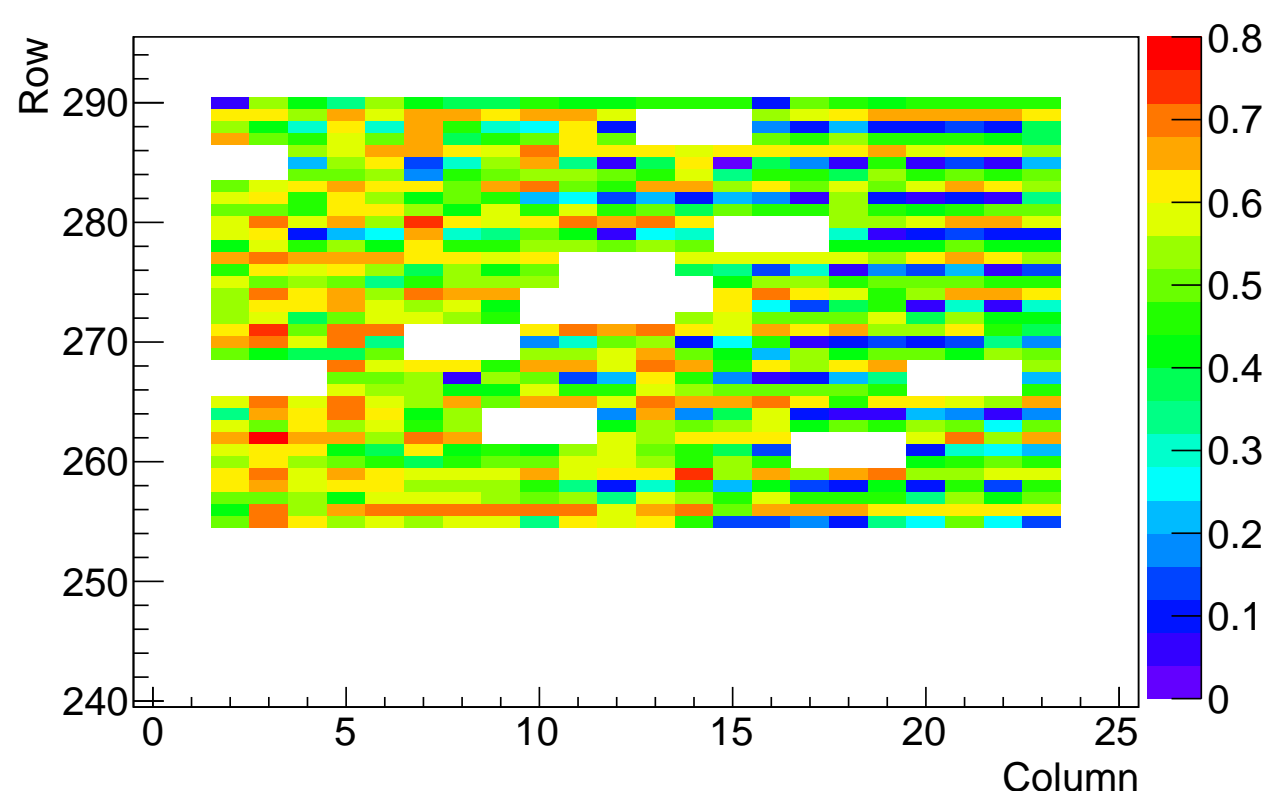

(a) Efficiency map - Likelihood Method.

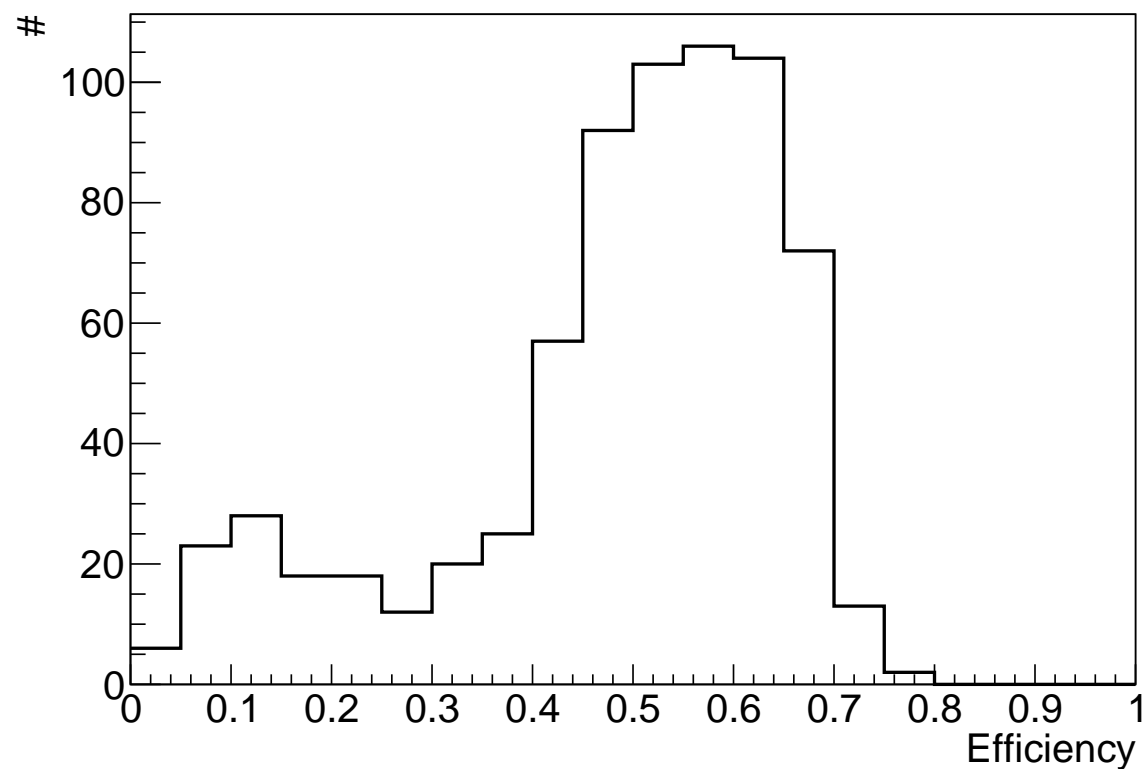

(b) Efficiency distribution - Likelihood Method.

Figure 6.52: Hit efficiency maps and distributions for the likelihood method. The pixel map shows the efficiency for every pixel. These values are histogramed in the distribution plots. 


\begin{tabular}{l|c|c} 
& Efficiency & Standard Deviation \\
\hline Cut Method & 0.39 & 0.14 \\
\hline Likelihood Method & 0.48 & 0.17
\end{tabular}

Table 6.6: Module hit efficiencies for the cut and the likelihood method. The high HV2FEI4V2 comparator threshold needs to be taken into account, while looking at the hit efficiency values. See text for details.

\begin{tabular}{l|c|c|c|c|c|c} 
& \multicolumn{2}{|c|}{ Subpixel 1 } & \multicolumn{2}{c|}{ Subpixel 2 } & \multicolumn{2}{c}{ Subpixel 3 } \\
& Efficiency & $\sigma$ & Efficiency & $\sigma$ & Efficiency & $\sigma$ \\
\hline Cut Method & 0.33 & 0.16 & 0.46 & 0.11 & 0.37 & 0.10 \\
\hline Likelihood Method & 0.35 & 0.20 & 0.62 & 0.06 & 0.49 & 0.08
\end{tabular}

Table 6.7: Hit efficiencies with their standard deviation $\sigma$ of the three subpixel types for the cut and the likelihood method.

in the hit efficiency between the three subpixel types is visible due to their intrinsic difference and the different threshold equivalent voltage (see Figure 6.18). Furthermore, the pixel maps show an efficiency gradient from the lower left to the upper right corner. A difference in the thickness of the glue layer, as already observed in Section 6.2.4, is a possible explanation for this effect. The signal amplitude in the upper right corner is lower and hence the hit efficiency. A higher signal in the upper right corner yields a larger efficiency.

Besides these observations, an increase in the overall hit efficiency from the cut method to the likelihood method is evident, confirming that the likelihood method, which uses ToT and Lvl1 information, improves the subpixel mapping. In Table 6.6 the module efficiencies are listed. The mean hit efficiency increases by $9 \%$ with the likelihood method. The upper limit of the hit efficiency distribution increases from approximately 0.65 to 0.80 .

Finally, the contributions of the three subpixel types to the residual and hit efficiency distribution was investigated. No significant difference is observed in the residual distribution of the three subpixel types (see Figures 6.53 and 6.54).

The hit efficiency distributions of the three subpixel types for the cut and the likelihood method are presented in Figure 6.55. The hit efficiency of subpixel type 2 is the largest, as it also illustrated in Figure 6.51a and 6.52a. A comparison of the efficiencies of the three subpixel types is shown in Table 6.7. The largest improvement is observed for the subpixel type 2 , for which the hit efficiency increases by $16 \%$ comparing the cut method to the likelihood method. Furthermore, the standard deviation of the hit efficiencies is smaller for the subpixel types 2 and 3 due to the better subpixel mapping. Due to the broad and flat distribution of subpixel type 1 there is no improvement other than an increase in the maximum pixel efficiency. 


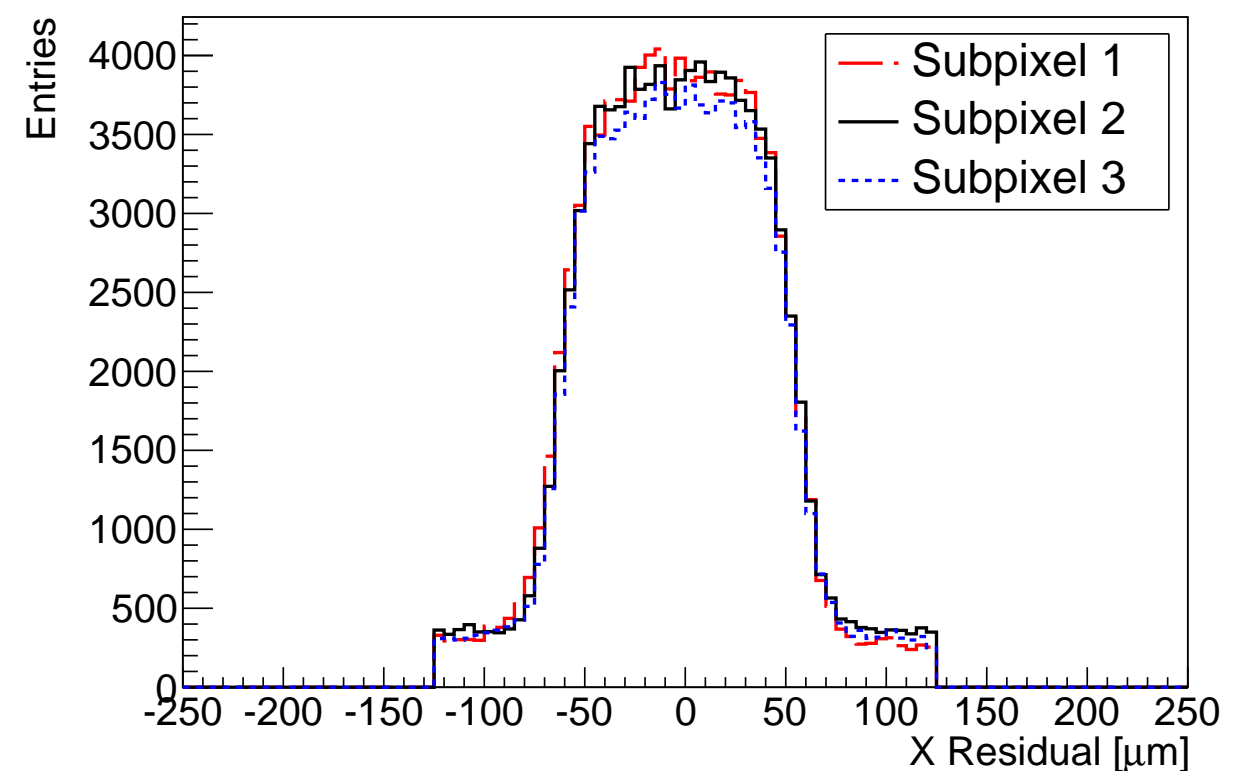

(a) $x$ residual - Cut Method.

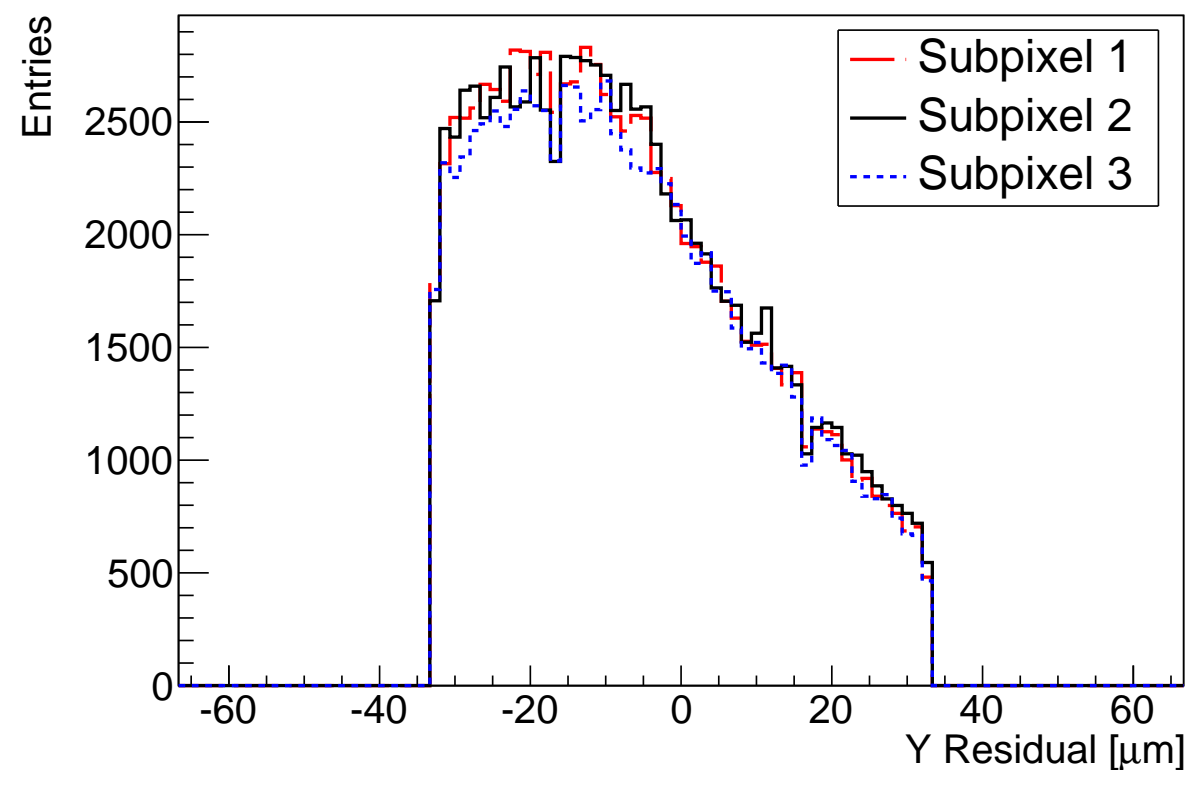

(b) y residual - Cut Method.

Figure 6.53: Contributions to the residual distributions of the three subpixel types for $x$ - and $y$-direction with the cut method. 


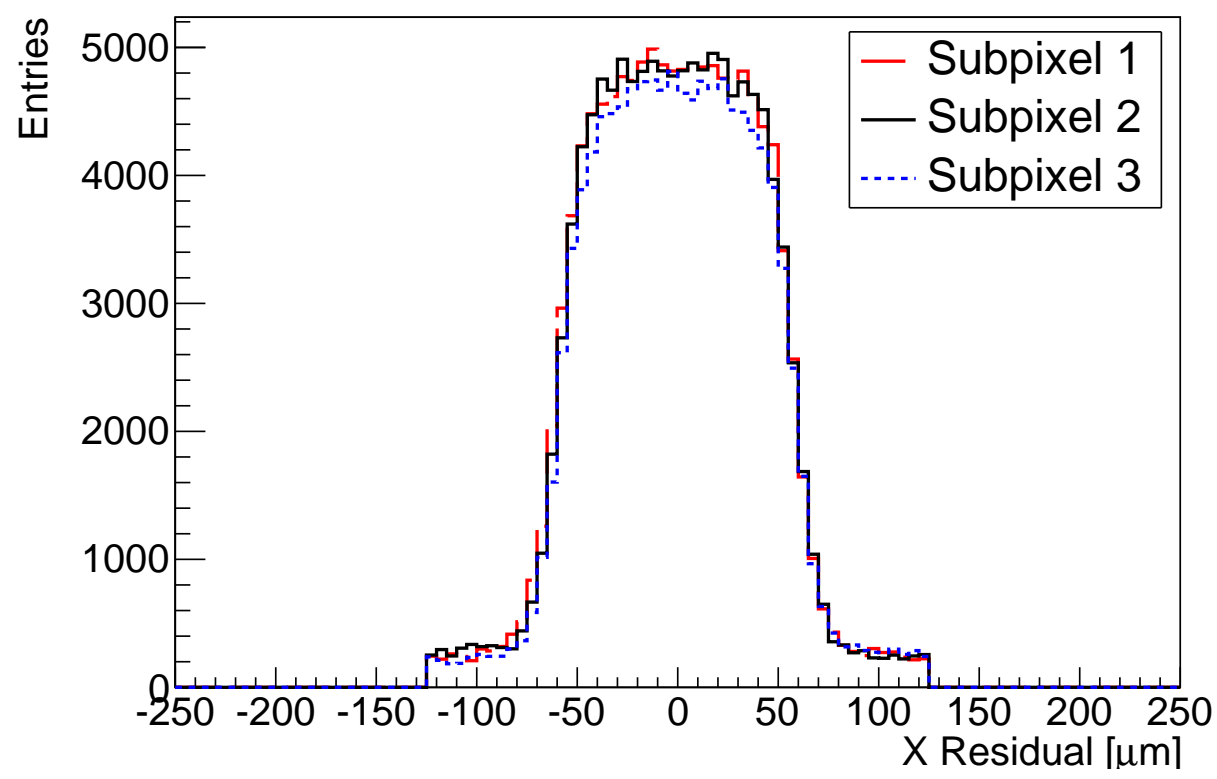

(a) $x$ residual - Likelihood Method.

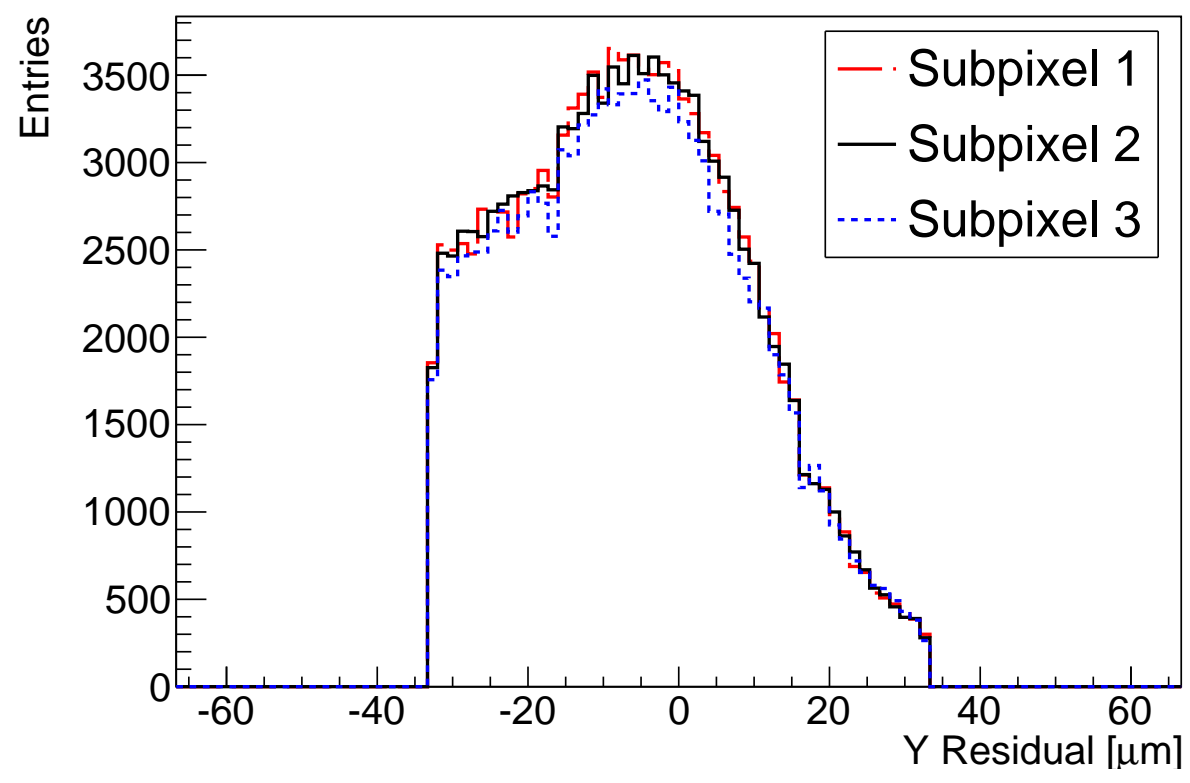

(b) y residual - Likelihood Method.

Figure 6.54: Contributions to the residual distributions of the three subpixel types for $x$ - and $y$-direction with the likelihood method. 


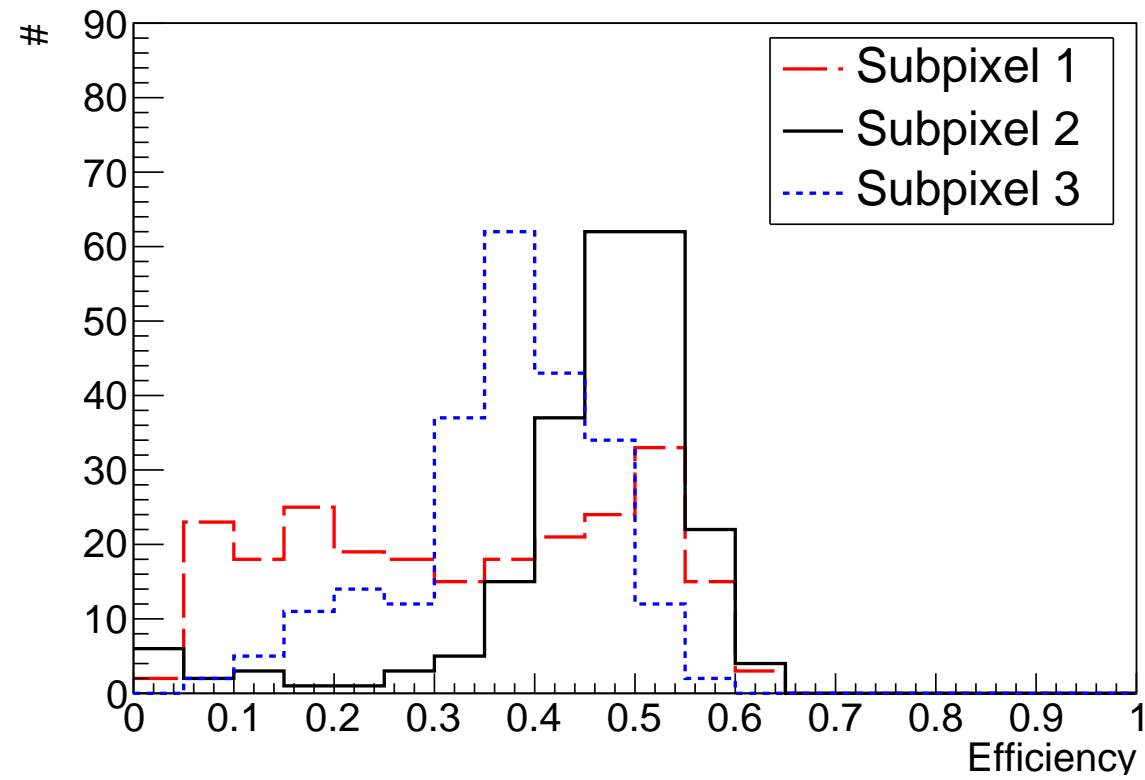

(a) Efficiency distribution - Cut Method.

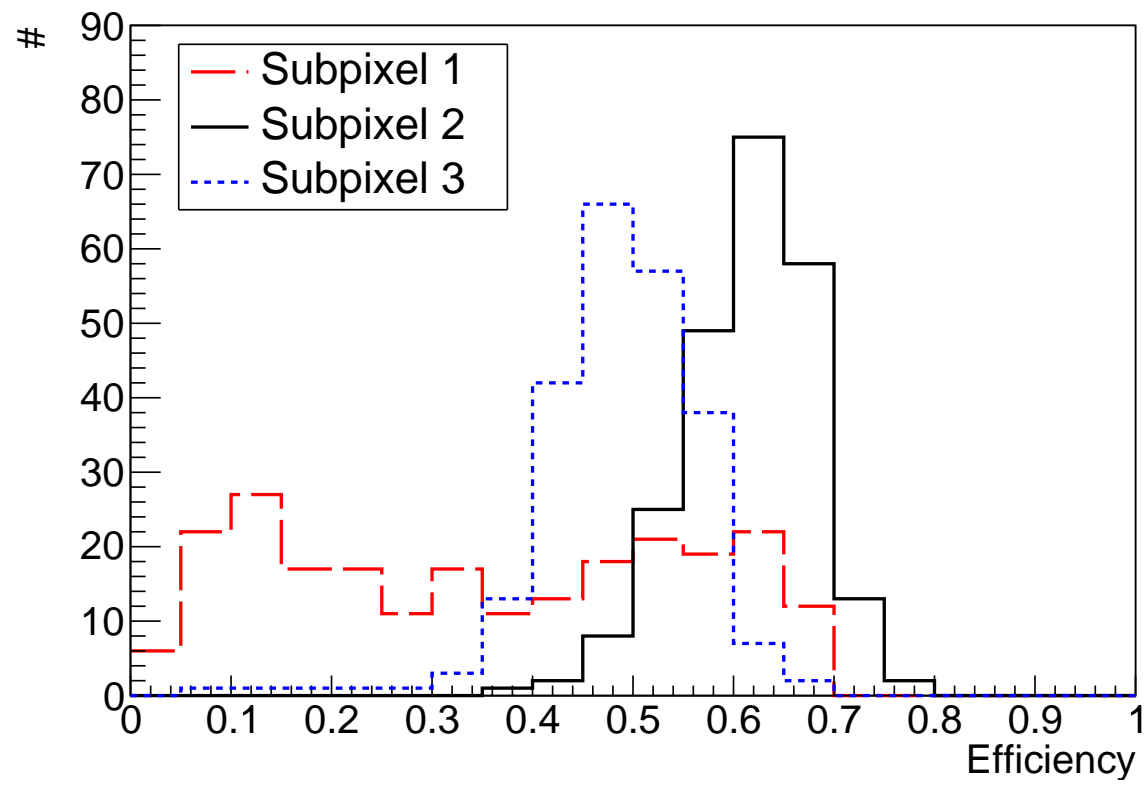

(b) Efficiency distribution - Likelihood Method.

Figure 6.55: Hit efficiency distributions for the three subpixel types for the cut (top) and the likelihood (bottom) method. 


\subsection{Hit Efficiency Studies with Complete Sensor}

In a second test beam period at DESY in March 2016, measurements with all subpixel types enabled were performed at an electron energy of $5 \mathrm{GeV}$. The set-up was the same as for the measurements with only one subpixel type enabled. For the HV2FEI4v2 the standard parameter configuration is used, but with the tuned VNOut1/2/3 DAC and FDAC setting. A sensor bias voltage of $-60 \mathrm{~V}$ is applied. The settings for the track reconstruction and the analyses are also the same as for the previous analyses. The cluster size is dominantly one. Approximately $2 \cdot 10^{6}$ triggers were recorded per parameter setting. The measurements were performed at room temperature.

For the subpixel mapping the likelihood method is used. As described in Section 6.4.3, approximately $5 \%$ of the hits are mapped to the wrong subpixel type. This error propagates to the hit efficiency. Hence, the relative uncertainty on the hit efficiency is dominated by the likelihood error.

The ToT and Lvl1 distributions of a test beam measurement with all subpixel types enabled are shown in Figures 6.56 and 6.57. In the ToT distribution (see Figure 6.56) the three

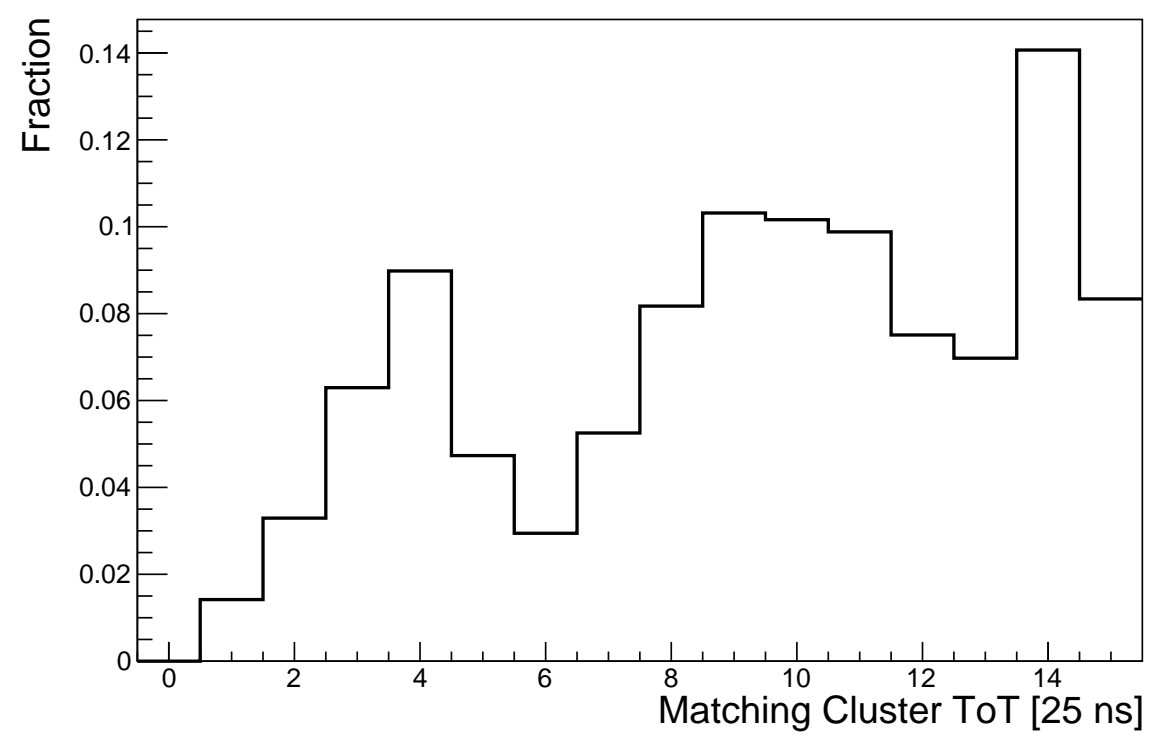

Figure 6.56: TOT distribution for a test beam measurement with all subpixel types enabled. The external threshold voltage is $0.89 \mathrm{~V}$ and the sensor bias voltage $-60 \mathrm{~V}$.

peaks for the three subpixel types are visible. The $L v l 1$ distribution (see Figure 6.57) is very broad, as expected, because it is composed of the contributions from the three subpixel types.

An external threshold voltage of $0.89 \mathrm{~V}$ is applied, which is the minimal stable voltage without further tuning, but still yields a high threshold with respect to the expected MIP 


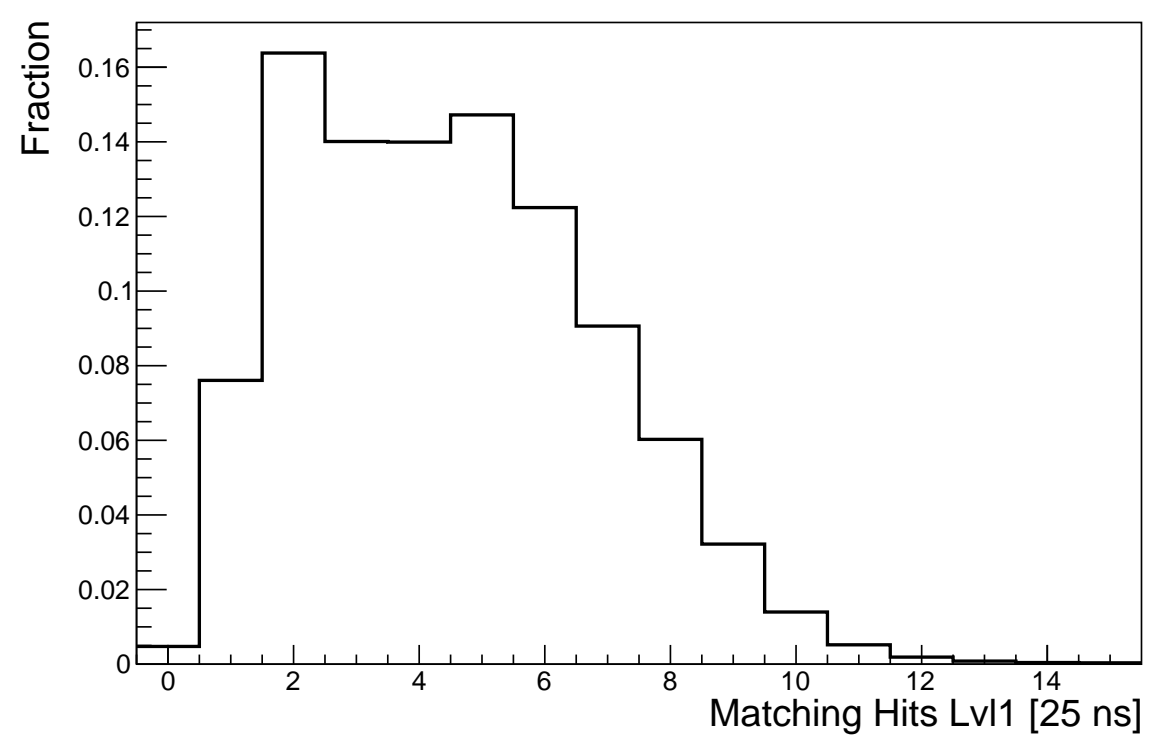

Figure 6.57: $L v / 1$ distribution for a test beam measurement with all subpixel types enabled. The external threshold voltage is $0.89 \mathrm{~V}$ and the sensor bias voltage $-60 \mathrm{~V}$.

signal.

In the pixel map (see Figure 6.58a) the different hit efficiencies of the three subpixel types are evident. Furthermore, a gradient in the hit efficiency from the left to the right side is visible. This gradient corresponds to the distribution of the average cluster ToT as seen in the measurements with only one subpixel type enabled (see Figure 6.28). On the right side the ToT signal is smaller and hence the efficiency.

The mean hit efficiencies and the standard deviation $\sigma$ are presented in Table 6.8. The

\begin{tabular}{c|c|c|c|c|c|c|c} 
Module average & \multicolumn{2}{|c|}{ Subpixel 1 } & \multicolumn{2}{c|}{ Subpixel 2 } & \multicolumn{2}{c}{ Subpixel 3 } \\
Efficiency & $\sigma$ & Efficiency & $\sigma$ & Efficiency & $\sigma$ & Efficiency & $\sigma$ \\
\hline 0.72 & 0.21 & 0.55 & 0.28 & 0.81 & 0.09 & 0.80 & 0.09
\end{tabular}

Table 6.8: Mean hit efficiencies and their standard deviation $\sigma$ for a test beam measurement with all subpixel types enabled at a sensor bias voltage of $-60 \mathrm{~V}$.

mean hit efficiency of subpixel type 2 and 3 is approximately 0.8 . The maximum pixel efficiency is contained in the bin between 0.95 to 1 . The total mean hit efficiency is decreased by the performance of subpixel type 1 . The hit efficiency distribution of subpixel type 1 (see Figure 6.58b) is very broad and the mean hit efficiency is lower than for the other subpixel types. This effect can be explained by two effects. First, the hit efficiency of subpixel type 1 was measured to be intrinsically lower compared to the others (see Section 6.3.1). Second, the low ToT response of subpixel type 1 together with the high threshold further reduces the efficiency. 


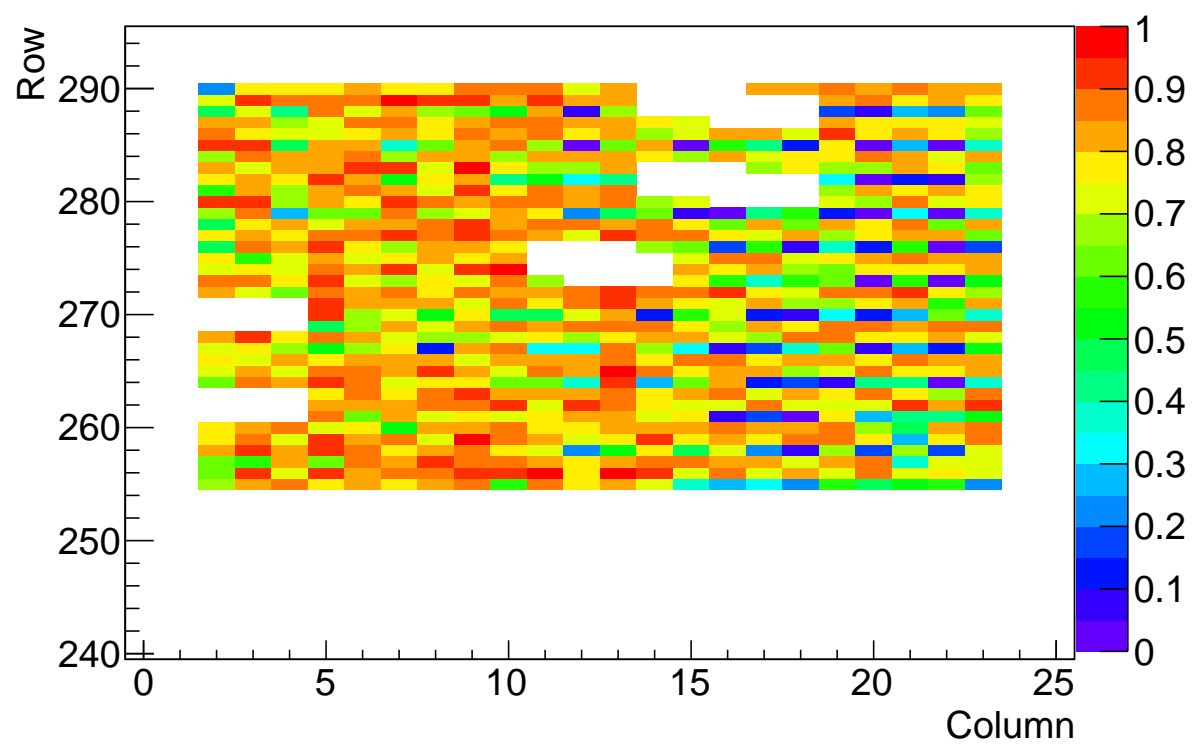

(a) Efficiency map.

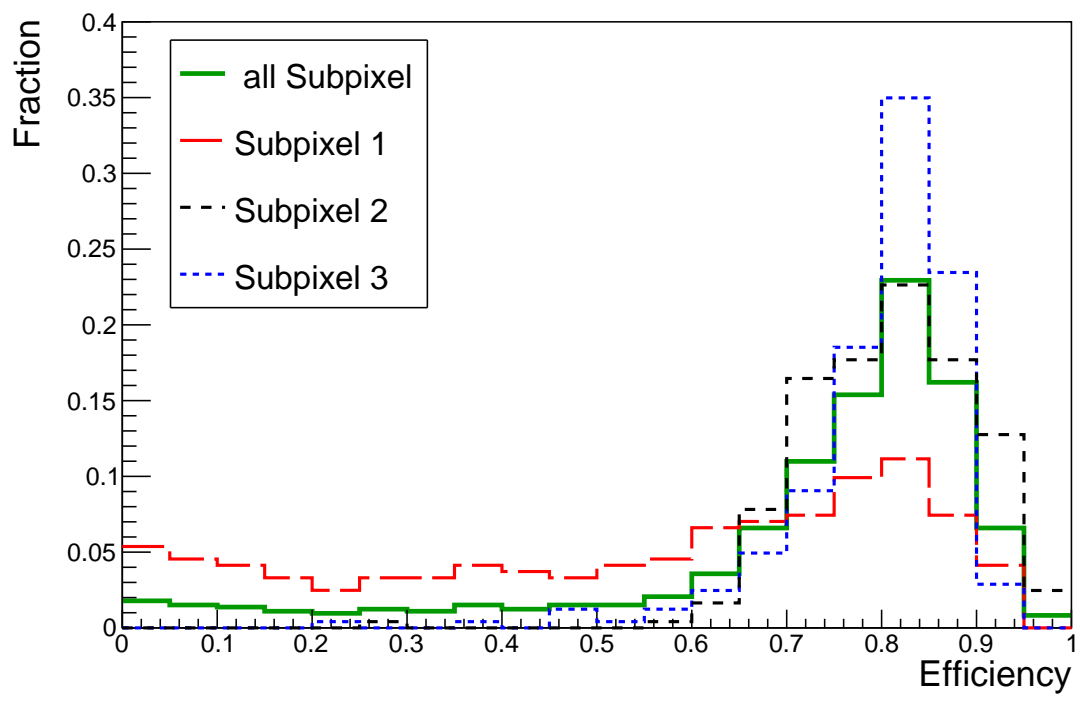

(b) Efficiency distribution.

Figure 6.58: Hit efficiency map and distribution for a test beam measurement with all subpixel types enabled. The external threshold voltage is $0.89 \mathrm{~V}$ and the sensor bias voltage $-60 \mathrm{~V}$.

The in-pixel efficiency plots, which are in the same geometrical orientation as described in Figure 6.32, are shown in Figure 6.59. The hit efficiency in the centre of the pixel is higher than at the edges due to charge sharing. Furthermore, the efficiency in the centre of the pixel is above 0.9 for subpixel type 2 and 3 . 


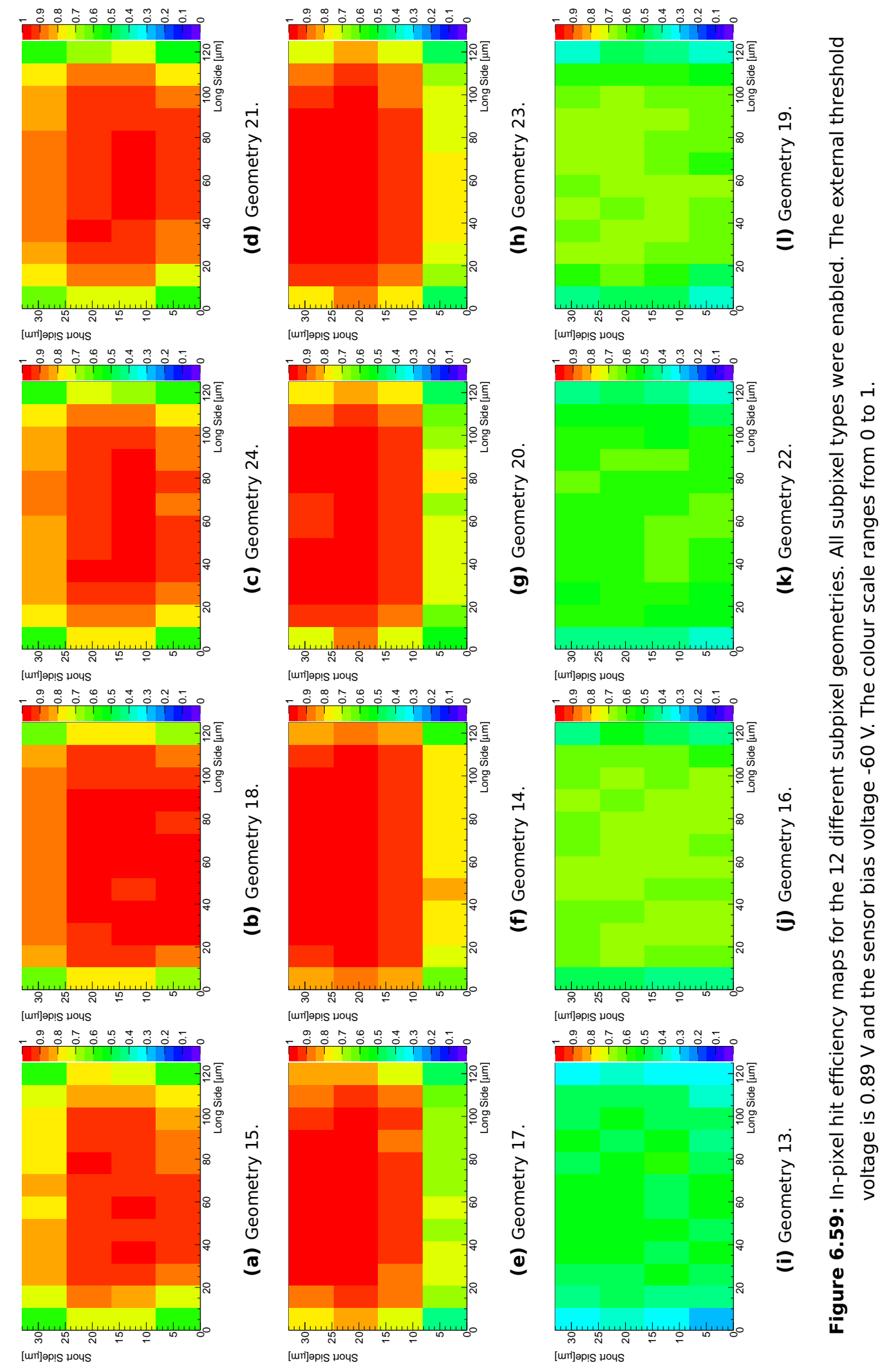




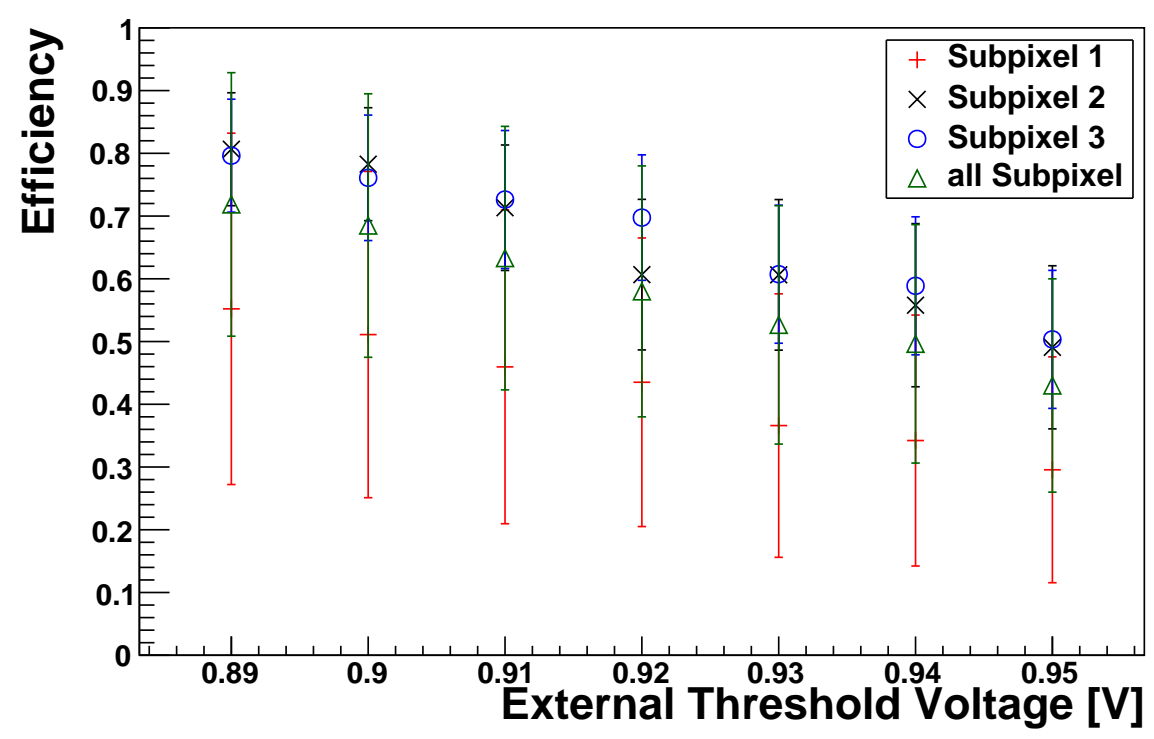

Figure 6.60: Hit efficiency as a function of the external threshold voltage at a sensor bias voltage of $-60 \mathrm{~V}$. All subpixel types are enabled. The error bar shows the standard deviation of the efficiency distribution.

Finally, the hit efficiency as a function of the external threshold voltage and the sensor bias voltage was investigated for the CCPD module. Figure 6.60 shows the hit efficiency for external threshold voltages between $0.89 \mathrm{~V}$ and $0.95 \mathrm{~V}$. As observed in the measurements with only one subpixel type enabled, the hit efficiency decreases with increasing external threshold voltage. The error bars show the standard deviation of the efficiency distribution. The large width of the distribution for subpixel type 1 can be seen. Separate plots per subpixel can be found in the Appendix (see Figure A.8).

Hit efficiency as a function of the sensor bias voltage is presented in Figure 6.61 for sensor bias voltages between $-30 \mathrm{~V}$ and $-80 \mathrm{~V}$. Again the large standard deviation of subpixel type 1 can be seen. No significant increase in the mean hit efficiency for a decreasing sensor bias voltage is seen. But if the hit efficiency distributions of -60 V (see Figure 6.58) and $-80 \mathrm{~V}$ (see Figure 6.62) are compared, the maximum bin of the distribution with $-80 \mathrm{~V}$ has more entries. In the Appendix (see Figure A.9) separate plots per subpixel can be found.

In summary, the behaviour of the hit efficiency as a function of the external threshold voltage and the sensor bias voltage is comparable with the previous measurements with only one enabled subpixel type. 


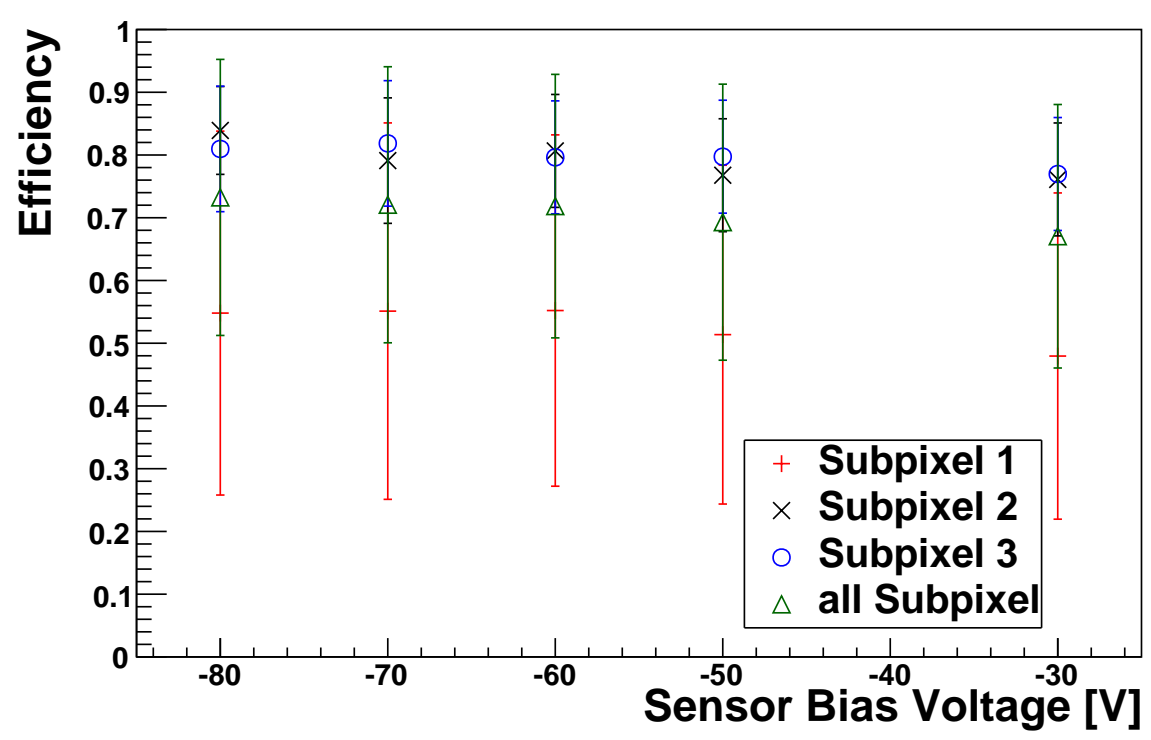

Figure 6.61: Hit efficiency as a function of the sensor bias voltage for an external threshold voltage of $0.89 \mathrm{~V}$. All subpixel types are enabled. The error bar shows the standard deviation of the efficiency distribution.

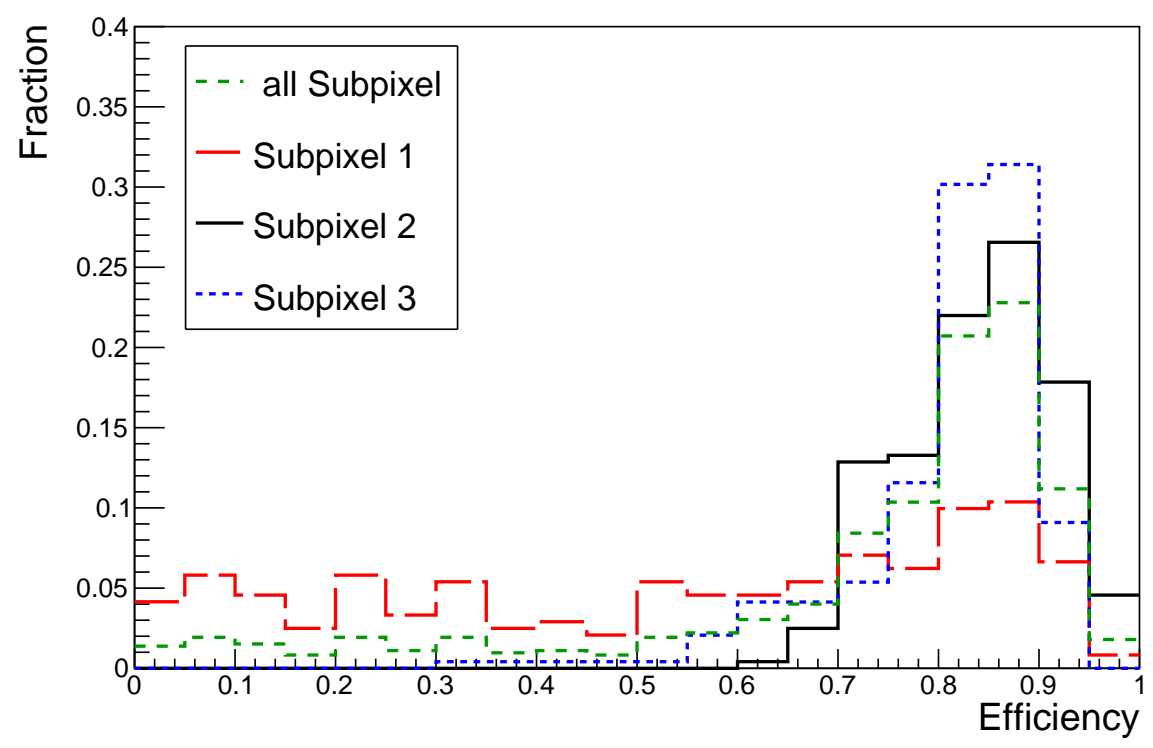

Figure 6.62: Hit efficiency map and distribution for a test beam measurement with all subpixel types enabled. The external threshold voltage is $0.89 \mathrm{~V}$ and the sensor bias voltage $-80 \mathrm{~V}$. 



\section{Conclusion and Outlook}

\subsection{Conclusion}

With the upgrade of the LHC to the HL-LHC, the luminosity will increase and it will be possible to get answers to questions left open in the Standard Model of particle physics. The physics goals include the electroweak and the Higgs boson physics, as well as the search of Supersymmetry and new physics processes.

A new ATLAS Inner Tracker will replace the current ATLAS Inner Detector to cope with the challenges arising from the estimated high luminosity of $7.5 \times 10^{34} \mathrm{~cm}^{-2} \mathrm{~s}^{-1}$ and the extremely high particle flux from up to 200 inelastic proton-proton collisions per bunch crossing. A finer granularity of the detectors is required to cope with the high occupancy and the trigger system needs input from the tracking system to identify key signatures of processes.

The detector environment changes with the distance to the interaction point and so do the detector requirements. In the outer pixel detector layer of ITk, a cost effective detector technology is needed due to the large area of $\mathscr{O}\left(10 \mathrm{~m}^{2}\right)$. In the context of this thesis two approaches to address this requirement were investigated and characterised: a four-chip module with a planar n-in-p sensor bump-bonded to four FE-I4 readout chips and a CCPD module consisting of an active HV2FEI4v2 CMOS sensor capacitively coupled to an FE-I4.

\subsubsection{Four-Chip Module}

In the outer layers of the future ITk pixel detector, the environment in terms of particle flux will be comparable to the current IBL environment. Consequently, the same detector technology is applicable, but a larger area needs to be covered. A four-chip module is read out by four FE-I4 readout chips, which were used for IBL and can cope with up to 2.5 MGy total ionising dose. The larger modules require less manual handling during production than modules with smaller area sensors and thus they are more cost effective. The studies in the framework of this thesis showed, that the performance of the four-chip modules is comparable with the IBL module performance. Even for a four times larger module, the discriminator threshold is tunable with a pixel dispersion approximately 50e at a desired threshold of 2000e. The threshold-to-noise ratio is 13.4, which yields a good separation.

The bump-bond quality of the module with a $200 \mu \mathrm{m}$ thick sensor was found to be good with only $0.1 \%$ bad connections. Together with a hit efficiency of above $98 \%$ four-chip modules are applicable for the requirements in the outer layers. 


\subsubsection{CCPD Module with HV2FEI4v2 Sensor}

In this thesis the CCPD module with the HV2FEI4v2 sensor, which is implemented in the AMS $180 \mathrm{~nm}$ high-voltage process, was characterised for the usage in the outer layers of the ITk. In the investigated CCPD module, every FE-I4 pixel is connected to three HV2FEI4 subpixels, whose position is encoded in the output signal amplitude. Due to the industrial production of the CMOS sensors, the CCPD module production is cost effective. The cost per square centimetre can be reduced by a factor of two compared with passive planar modules.

In the context of this thesis, the USBpix readout system and its software STcontrol were expanded to provide the CMOS sensor specific functionalities. The simultaneous access of the configuration parameters of the readout chip and HV2FEI4v2 sensor during one scan and a tuning of the readout chip response to the signal of the HV2FEI4v2 was implemented.

Several HV2FEI4v2 parameter studies were preformed with a focus on the comparator threshold, which was found to be mainly influenced by the external threshold voltage and the feedback parameter VNFB. The threshold-to-noise was found to be 6.25 , which is half the ratio of the four-chip module, but still sufficient for separation. The minimum stable external threshold voltage was found to correspond to a threshold charge of approximately $1000 e$, which is about the MIP signal in $10 \mu \mathrm{m}$ of silicon.

With the configuration sequence in STcontrol it is possible to set a different in-pixel threshold setting to the HV2FEI4v2 pixels for the first time. But the measurements showed that the in-pixel parameter does not influence the threshold in a well-defined way, which makes in-pixel tuning very difficult.

The second focus of the HV2FEI4v2 parameter studies was the adjustment of the ToT response of the FE-I4 to the sensor signal for a successful subpixel decoding. The parameter range, in which a significant change of the output voltage is possible, was found to be small, which makes subpixel decoding a complicated task.

A gradient in the FE-I4 feedback tuning result and the subsequent measurement of the height difference between the sensor surface and the FEI4 revealed a possible inhomogeneous thickness of the glue layer and thus a different coupling capacitance. For the application in the ATLAS ITk a homogeneous coupling capacitance needs to be achieved.

Furthermore, test beam measurements were performed with only one subpixel type enabled and with all three subpixel types enabled. A maximum likelihood method was introduced for subpixel decoding, which was found to yield less wrongly mapped hits and an improved efficiency compared to a cut based method.

Due to the subpixel structure the spatial resolution of $35.6 \pm 0.1 \mu \mathrm{m}$ for the x-direction and $15.65 \pm 0.06 \mu \mathrm{m}$ for the $\mathrm{y}$-direction was achieved.

The timing of the CCPD module was investigated and a very broad Lvl1 distribution was evident. For the usage in the ATLAS Experiment the width of the $L v l 1$ distribution needs to be reduced. The external threshold voltage and the feedback parameter VNFB influence the $L v l 1$ distribution, but the required width of one bin cannot be achieved.

As a representative of the three subpixel types, subpixel type 1 was characterised and the 
mean hit efficiency was found to be $79 \%$ with a standard deviation of $5 \%$. The mean hit efficiency decreases with an increasing external threshold voltage, but the expected increase with a decreasing sensor bias voltage was not observed. A possible explanation is that the charge is collected by a mixture of drift and diffusion.

Finally, the mean hit efficiency with all subpixel types enabled was investigated and found to be $72 \%$ with a large standard deviation of $21 \%$. The mean hit efficiency of subpixel types 2 and 3 is approximately $80 \%$. The measurement of the in-pixel efficiency showed, that the hit efficiency in the centre of the pixel is higher than at the edges due to charge sharing.

The HV2FEI4v2 is a very early prototype towards a CCPD module for the ITk. The concept of the subpixel de- and encoding works and the pixel resolution can be improved even with a readout chip with larger pixels. The mean hit efficiency of the module is with $72 \%$ too low, but a lower HV2FEI4 comparator threshold can solve this problem. Furthermore, a homogeneous glue layer thickness is necessary for a uniform signal transmission. The largest improvements are required in the timing of the chip. With the current timing resolution, the HV2FEI4v2 is not suitable for ITk.

\subsection{Outlook}

The HV2FEI4v2 is an early version of several prototypes and newer designs address the problems of the timing and the tuning possibilities. Studies investigating the concept of the interconnection of readout chip and sensor are ongoing. Extensive irradiation campaigns are performed to demonstrate the radiation hardness of the active CMOS sensors and the CCPD modules. Furthermore, the transient current technique is used to investigate the development of the depletion zone in more detail. The in-time efficiency must be studied in measurements with laser injection. These measurements could not be performed in the context of this thesis, because the sensor backside was completely metallised.

Active CMOS sensors as monolithic pixel detectors are also a possible option for ITk. They have the advantage of a smaller material budget, but the fast readout speed needs to be demonstrated for the upcoming Level 0 trigger rate of $500 \mathrm{kHz}$.

The decision on a baseline sensor technology for the pixel detector of the ATLAS Inner Tracker needs to be made in 2017 at the time of the Technical Design Report. The active CMOS sensor technology has to show the suitability for the ITk to become an option by 2017, which is a very challenging task. 



\section{A Additional Plots}

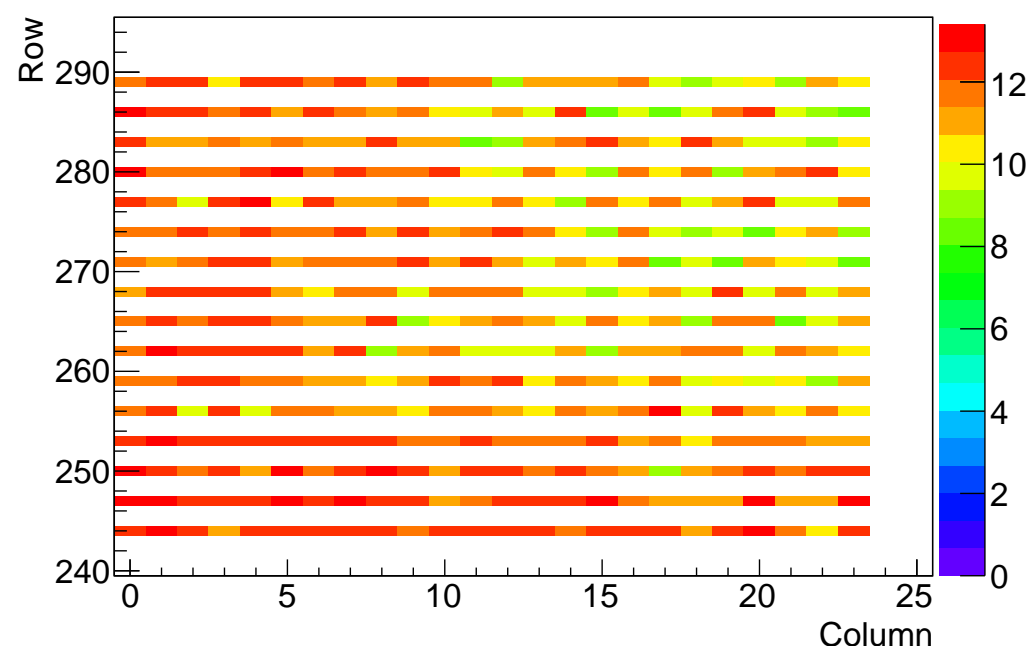

Figure A.1: Pixel map of average cluster ToT for one enabled subpixel type. Only subpixel type 2 is enabled and the VNOut2 DAC value is set to 60.

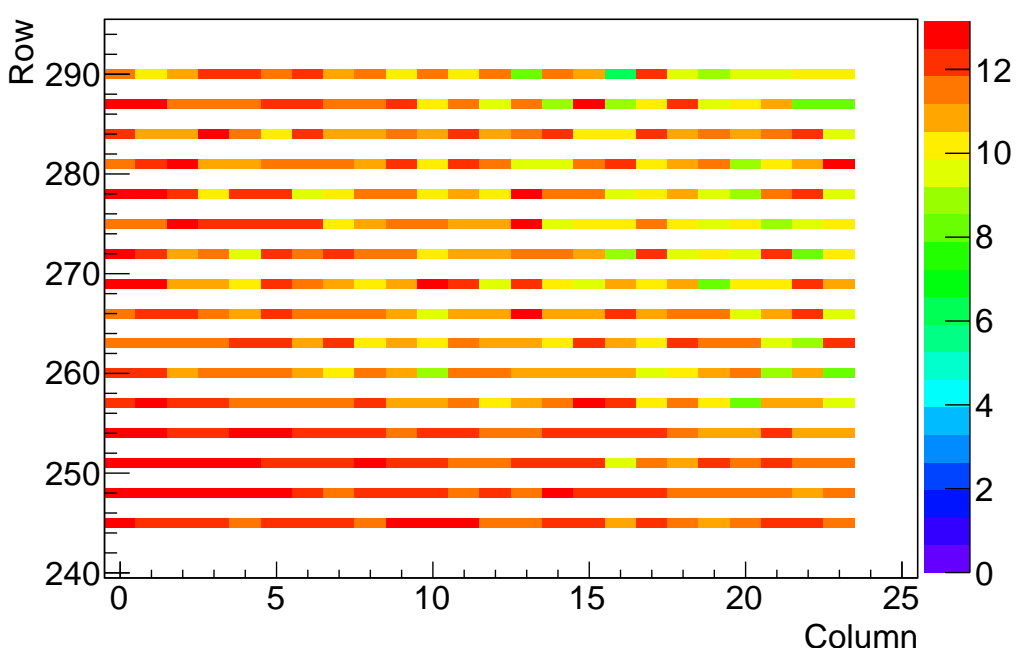

Figure A.2: Pixel map of average cluster ToT for one enabled subpixel type. Only subpixel type 3 is enabled and the VNOut3 DAC value is set to 60 . 


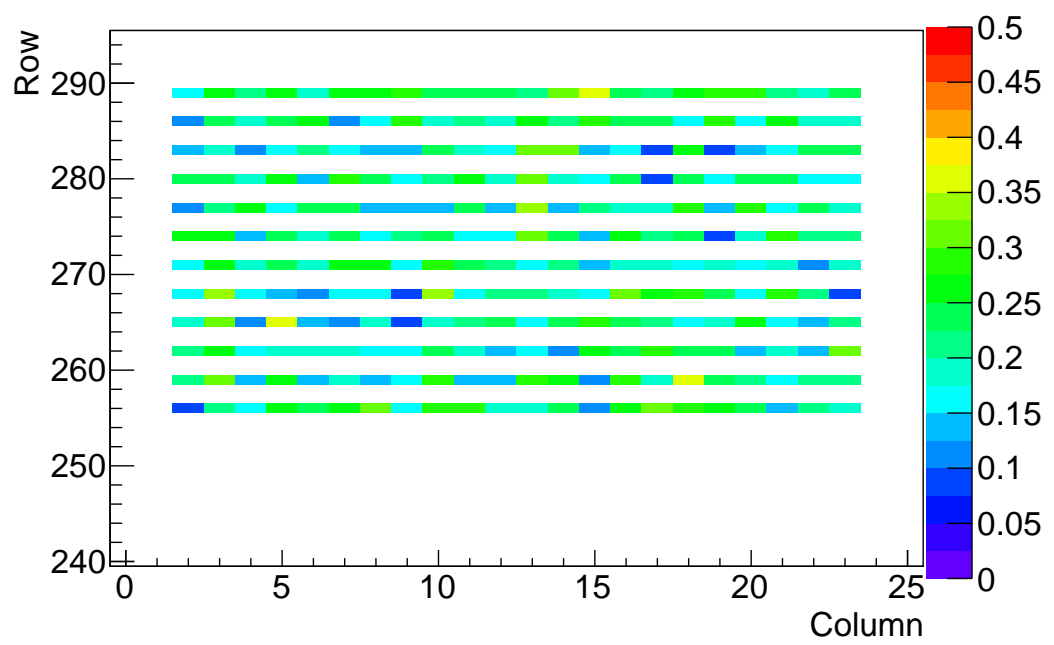

Figure A.3: Hit efficiency pixel map for one enabled subpixel type. Only subpixel type 2 is enabled. The efficiency scale ranges from 0 to 0.5 .

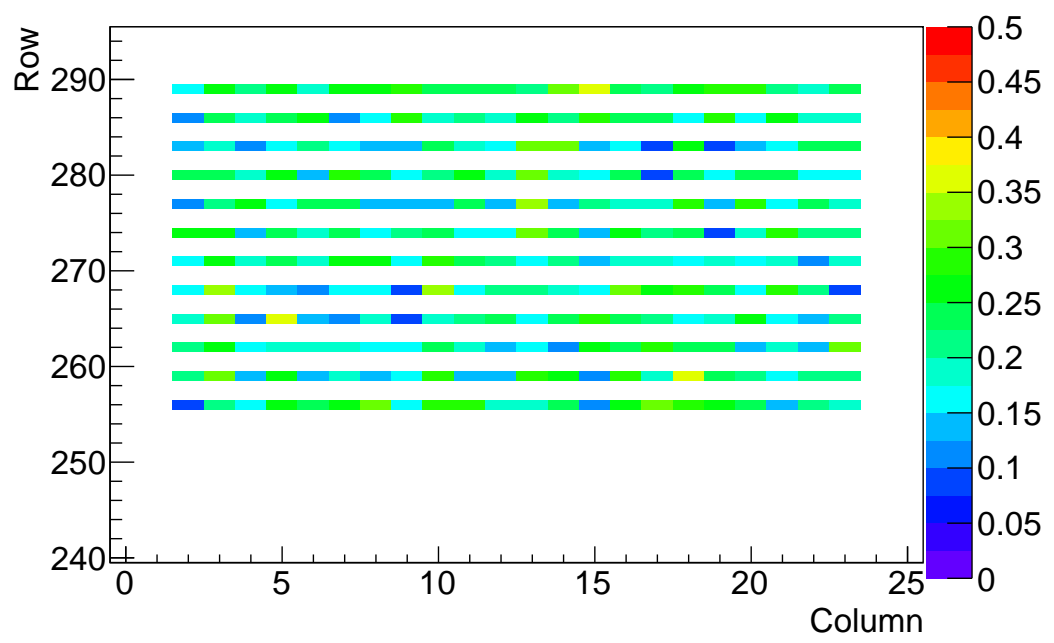

Figure A.4: Hit efficiency pixel map for one enabled subpixel type. Only subpixel type 3 is enabled. The efficiency scale ranges from 0 to 0.5 . 


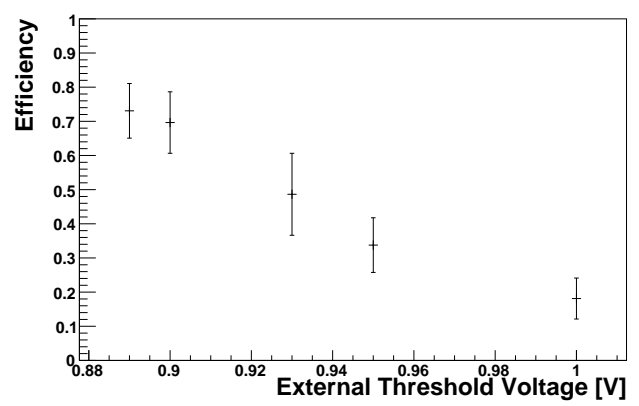

(a) $V_{\text {bias }}=-10 \mathrm{~V}$.

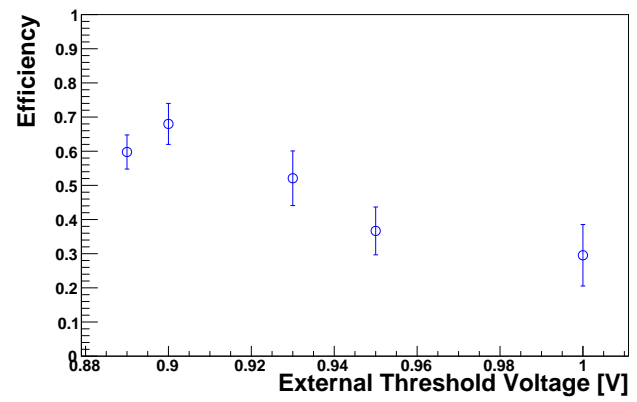

(c) $V_{\text {bias }}=-30 \mathrm{~V}$.

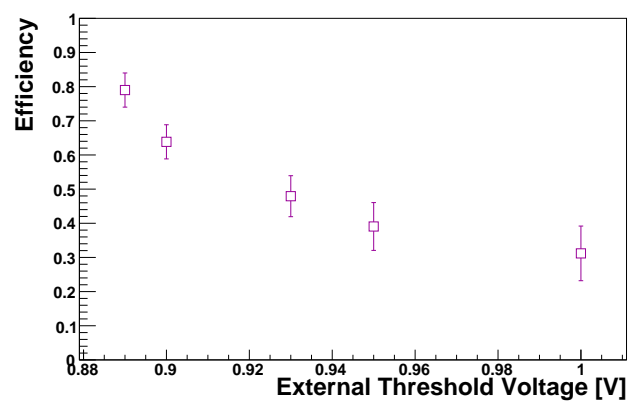

(e) $V_{\text {bias }}=-50 \mathrm{~V}$.

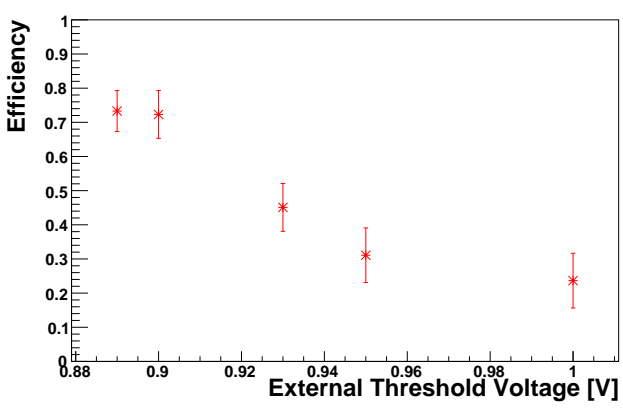

(b) $V_{\text {bias }}=-20 \mathrm{~V}$.

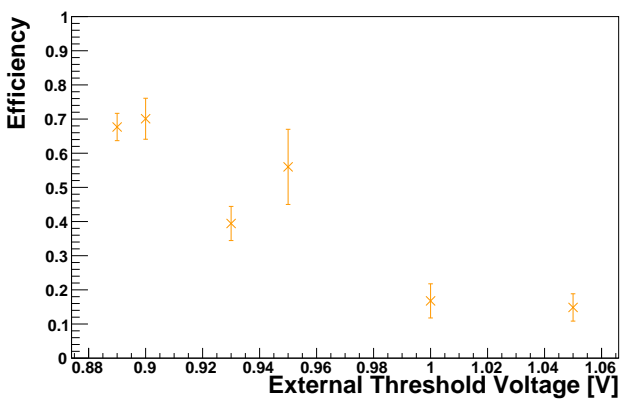

(d) $V_{\text {bias }}=-40 \mathrm{~V}$.

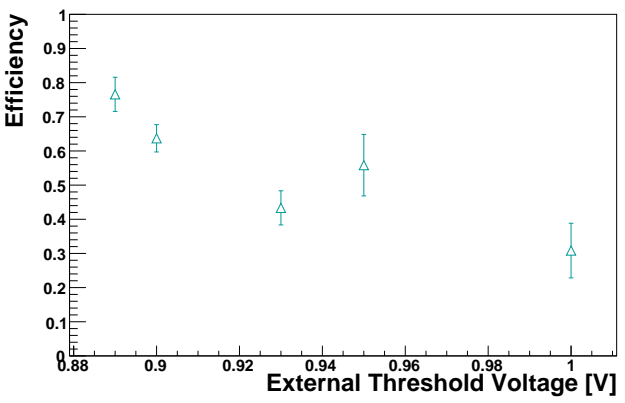

(f) $V_{\text {bias }}=-60 \mathrm{~V}$.

Figure A.5: Hit efficiency as a function of the external threshold voltage for sensor bias voltages between $-10 \mathrm{~V}$ and $-60 \mathrm{~V}$. Only subpixel type 1 is enabled. 


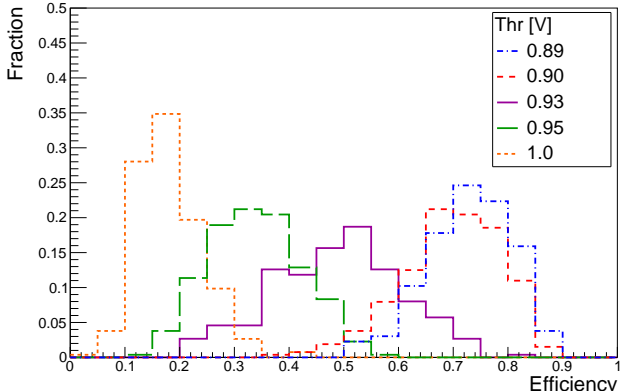

(a) $V_{\text {bias }}=-10 \mathrm{~V}$.

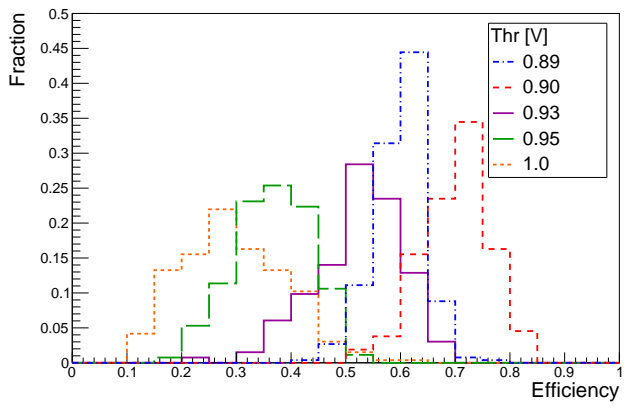

(c) $V_{\text {bias }}=-30 \mathrm{~V}$.

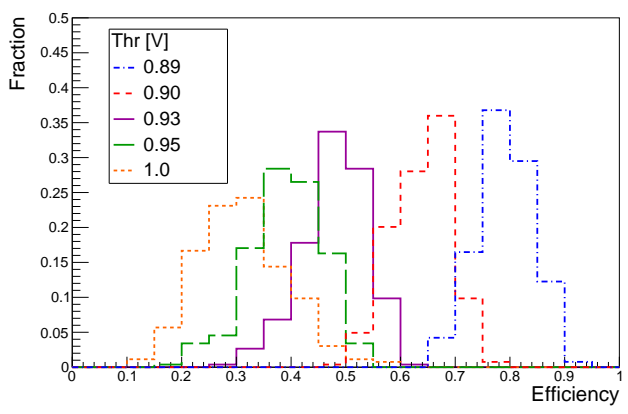

(e) $V_{\text {bias }}=-50 \mathrm{~V}$.

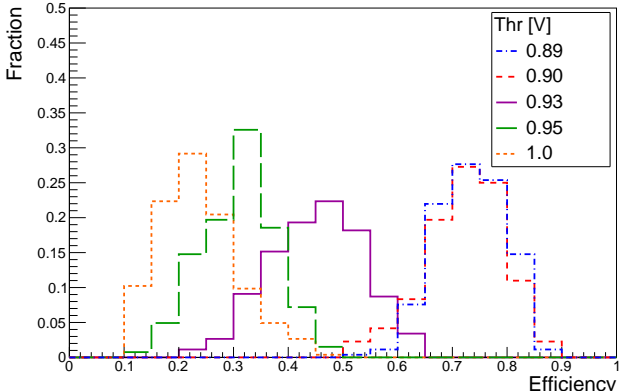

(b) $V_{\text {bias }}=-20 \mathrm{~V}$.

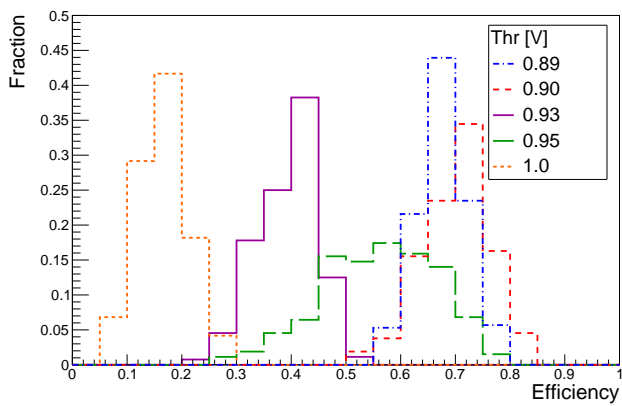

(d) $V_{\text {bias }}=-40 \mathrm{~V}$

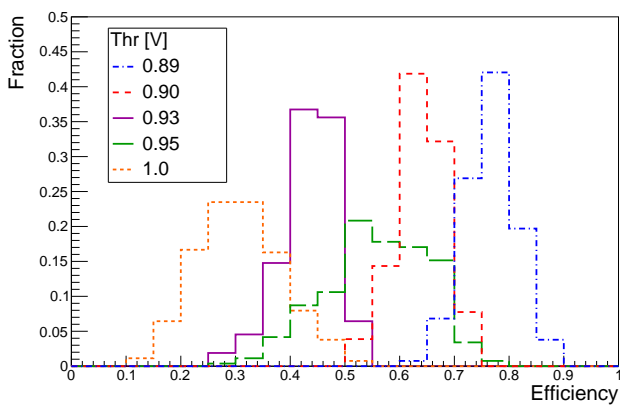

(f) $V_{\text {bias }}=-60 \mathrm{~V}$.

Figure A.6: Hit efficiency distributions for different external threshold voltages for sensor bias voltages between $-10 \mathrm{~V}$ and $-60 \mathrm{~V}$. Only subpixel type 1 is enabled. 


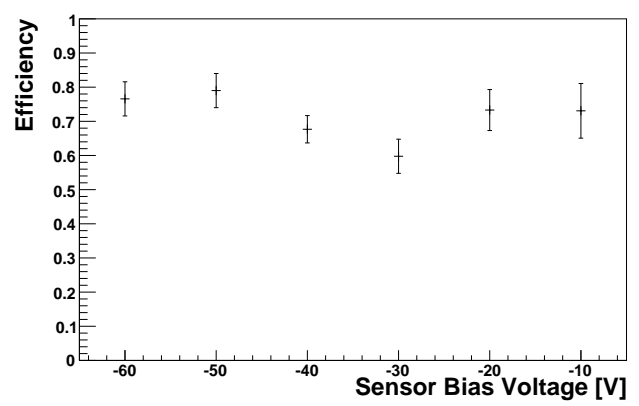

(a) $\mathrm{Thr}=0.89 \mathrm{~V}$.

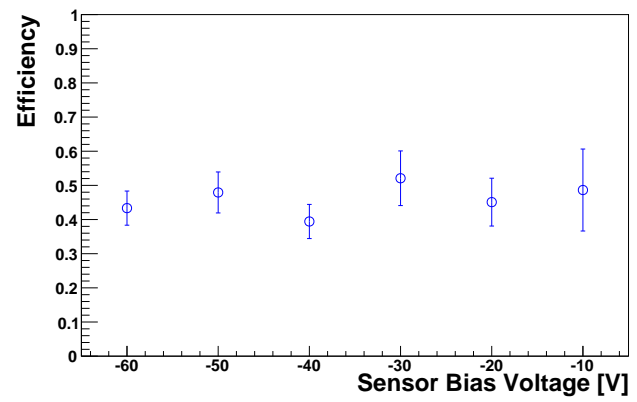

(c) $\mathrm{Thr}=0.93 \mathrm{~V}$.

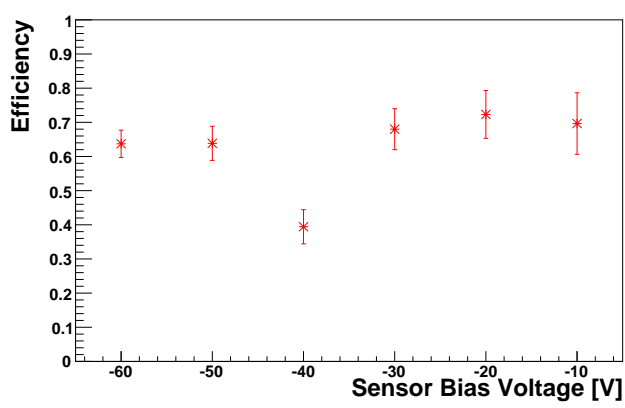

(b) $\mathrm{Thr}=0.90 \mathrm{~V}$.

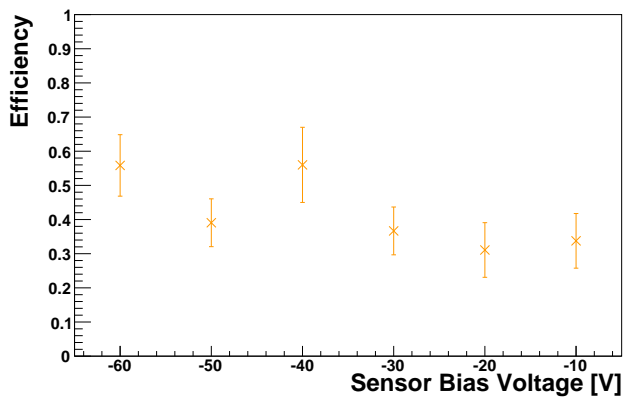

(d) $\mathrm{Thr}=0.95 \mathrm{~V}$.

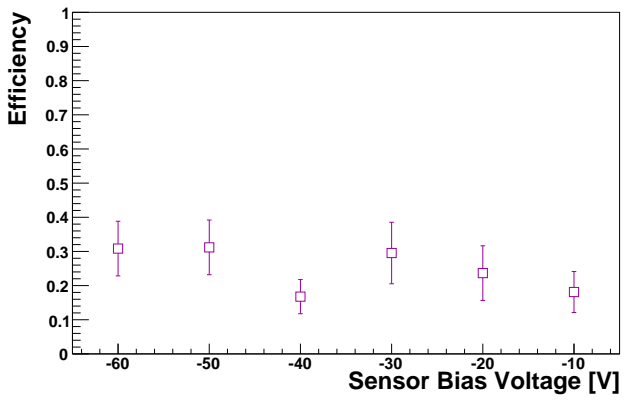

(e) $\mathrm{Thr}=1.0 \mathrm{~V}$.

Figure A.7: Hit efficiency as a function of the sensor bias voltage for external threshold voltages between $0.89 \mathrm{~V}$ and $1.0 \mathrm{~V}$. Only subpixel type 1 is enabled. 


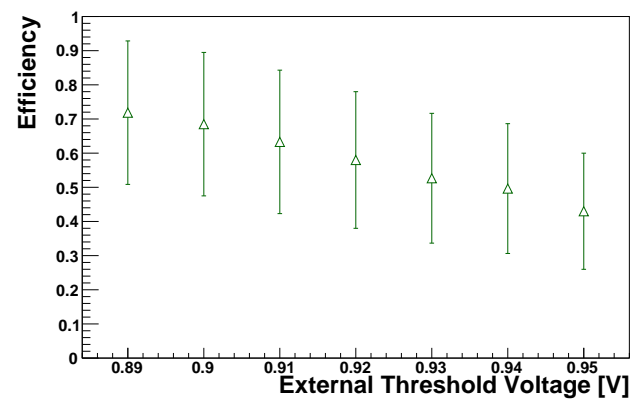

(a) All subpixels.

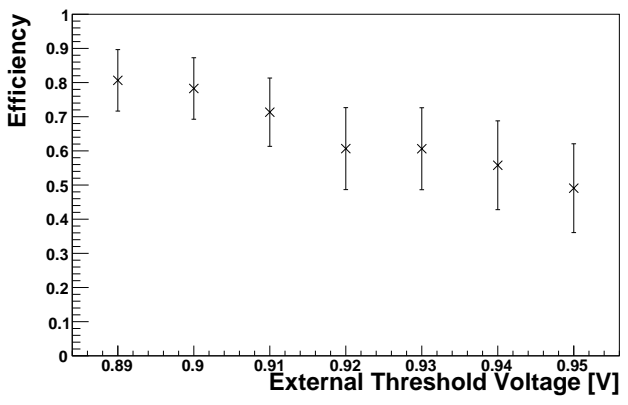

(c) Subpixel 2.

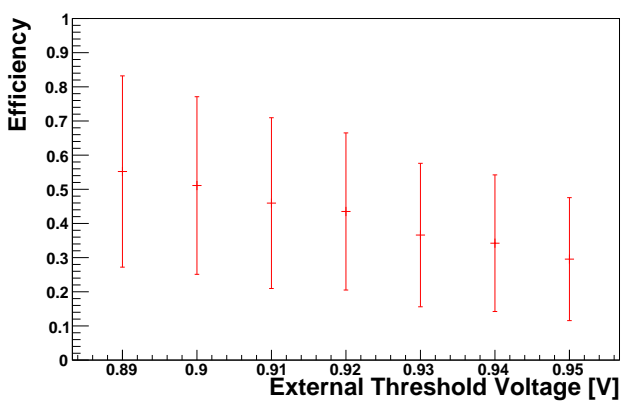

(b) Subpixel 1 .

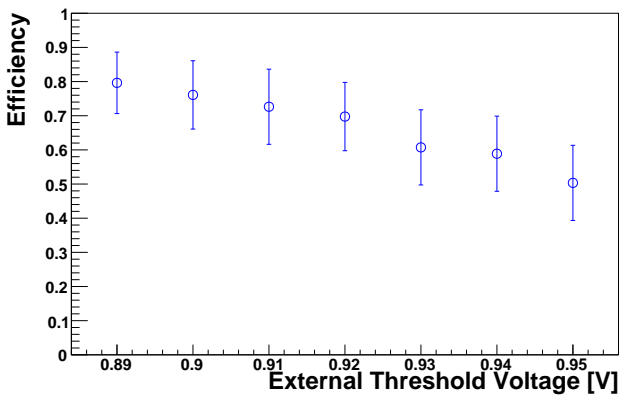

(d) Subpixel 3.

Figure A.8: Hit efficiency as a function of the external threshold voltage at a sensor bias voltage of $-60 \mathrm{~V}$. All subpixel types are enabled. The error bar states the standard deviation of the efficiency distribution. 


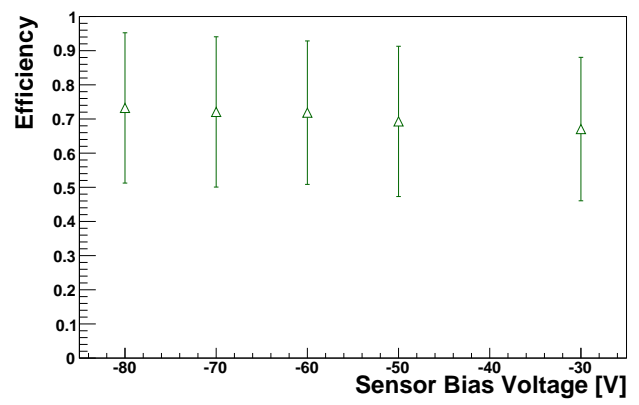

(a) All subpixels

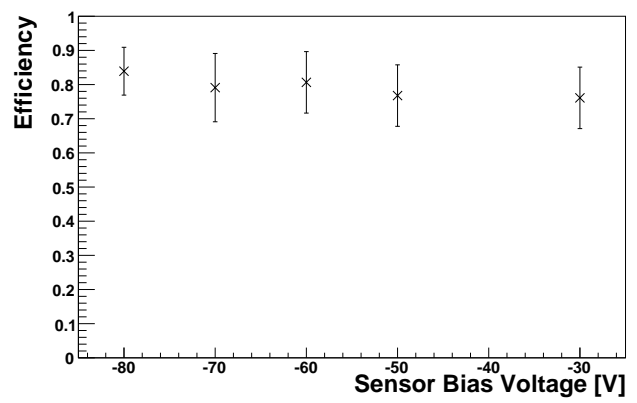

(c) Subpixel 2 .

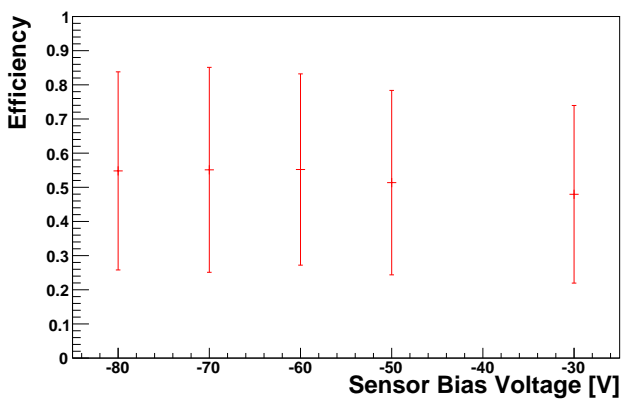

(b) Subpixel 1.

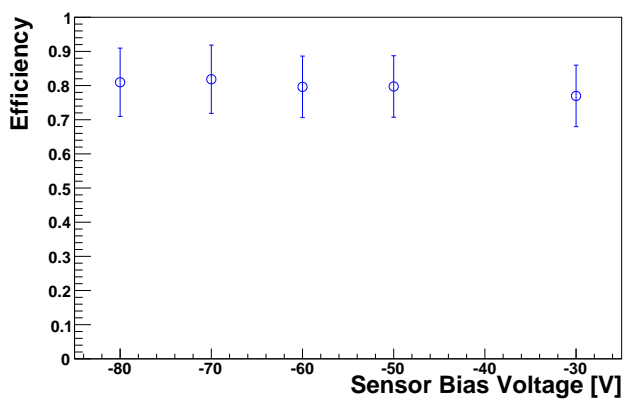

(d) Subpixel 3.

Figure A.9: Hit efficiency as a function of the sensor bias voltage at an external threshold voltage of $0.89 \mathrm{~V}$. All subpixel types are enabled. The error bars state the standard deviation of the efficiency distribution. 



\section{Bibliography}

[1] Nobelprize.org, C.T.R. Wilson - Facts, http://www.nobelprize.org/nobel_ prizes/physics/laureates/1927/wilson-facts.html. website, visited on June 13th, 2016.

[2] S. L. Glashow, Partial Symmetries of Weak Interactions, Nucl. Phys. 22 (1961) 579.

[3] S. Weinberg, A Model of Leptons, Phys. Rev. Lett. 19 (1967) 1264.

[4] A. Salam, Weak and Electromagnetic Interactions, Conf. Proc. C680519 (1968) 367.

[5] D. J. Gross and F. Wilczek, Ultraviolet Behavior of Non-Abelian Gauge Theories, Phys. Rev. Lett. 30 (1973) 1343.

[6] Particle Data Group Collaboration, K. A. Olive et al., Review of Particle Physics, Chin. Phys. C38 (2014) 090001.

[7] ATLAS Collaboration, Observation of a new particle in the search for the Standard Model Higgs boson with the ATLAS detector at the LHC, Phys. Lett. B 716 (2012) no. 1,1 .

[8] CMS Collaboration, Observation of a new boson at a mass of $125 \mathrm{GeV}$ with the CMS experiment at the LHC, Phys. Lett. B 716 (2012) no. 1, 30.

[9] P. W. Higgs, Broken Symmetries and the Masses of Gauge Bosons, Phys. Rev. Lett. 13 (1964) 508.

[10] F. Englert and R. Brout, Broken Symmetry and the Mass of Gauge Vector Mesons, Phys. Rev. Lett. 13 (1964) 321.

[11] G. S. Guralnik, C. R. Hagen, and T. W. B. Kibble, Global Conservation Laws and Massless Particles, Phys. Rev. Lett. 13 (1964) 585.

[12] SNO Collaboration, Q. R. Ahmad et al., Direct Evidence for Neutrino Flavor Transformation from Neutral-Current Interactions in the Sudbury Neutrino Observatory, Phys. Rev. Lett. 89 (2002) 011301.

[13] Super-Kamiokande Collaboration, Y. Fukuda et al., Evidence for Oscillation of Atmospheric Neutrinos, Phys. Rev. Lett. 81 (1998) 1562.

[14] V. C. Rubin and W. K. Ford, Jr., Rotation of the Andromeda Nebula from a Spectroscopic Survey of Emission Regions, Astrophys. J. 159 (1970) 379.

[15] D. Clowe et al., A Direct Empirical Proof of the Existence of Dark Matter, Astrophys. J. Lett. 648 (2006) L109. 
[16] D. N. Spergel et al., First-Year Wilkinson Microwave Anisotropy Probe (WMAP) Observations: Determination of Cosmological Parameters, Astrophys. J. Suppl. S. 148 (2003) no. 1, 175.

[17] O. S. Brüning et al., LHC Design Report. CERN, Geneva, 2004.

[18] ATLAS Collaboration, Luminosity Public Results, https://twiki.cern.ch/ twiki/bin/view/AtlasPublic/LuminosityPublicResults. website, visited on February 16th, 2016.

[19] ATLAS Collaboration, Luminosity Public Results Run 2, https ://twiki.cern.ch/ twiki/bin/view/AtlasPublic/LuminosityPublicResultsRun2. website, visited on June 23rd, 2016.

[20] ATLAS Collaboration, The ATLAS Experiment at the CERN Large Hadron Collider, JINST 3 (2008) S08003.

[21] ATLAS Collaboration, ATLAS Phase-II Upgrade Scoping Document, Tech. Rep. CERN-LHCC-2015-020. LHCC-G-166, CERN, Geneva, 2015.

[22] High Luminosity Large Hadron Collider Collaboration, The HL-LHC project, http://hilumilhc.web.cern.ch/about/hl-lhc-project. website, visited on February 24th, 2016.

[23] ATLAS Collaboration, Letter of Intent for the Phase-II Upgrade of the ATLAS Experiment, Tech. Rep. CERN-LHCC-2012-022. LHCC-I-023, CERN, Geneva, 2012.

[24] H. Bethe, Zur Theorie des Durchgangs schneller Korpuskularstrahlen durch Materie, Ann. Phys. 397 (1930) 325.

[25] F. Bloch, Bremsvermögen von Atomen mit mehreren Elektronen, Z. Phys. 81 (1933) 363.

[26] L. Landau, On the energy loss of fast particles by ionization, J. Phys.(USSR) 8 (1944) 201.

[27] E. Rutherford, The scattering of alpha and beta particles by matter and the structure of the atom, Phil. Mag. 21 (1911) 669.

[28] J. Große-Knetter, Vertex Measurement at a Hadron Collider - The ATLAS Pixel Detector. Habilitation, Universität Bonn, 2008.

[29] W. Leo, Techniques for nuclear and particle physics experiments: a how-to approach. Springer, 1994.

[30] P. P. Altermatt et al., Reassessment of the intrinsic carrier density in crystalline silicon in view of band-gap narrowing, J. Appl. Phys. 93 (2003) no. 3, 1598.

[31] H. Kolanoski and N. Wermes, Teilchendetektoren - Grundlagen und Anwendungen. Springer Spektrum, 2016. 
[32] S. Ramo, Currents Induced by Electron Motion, Proc. IRE 27 (1939) no. 9, 584.

[33] J. Srour, C. Marshall, and P. Marshall, Review of displacement damage effects in silicon devices, IEEE Trans. Nucl. Sci. 50 (2003) 653.

[34] M. Moll, Radiation damage in silicon particle detectors: microscopic defects and macroscopic properties. PhD thesis, DESY-THESIS-1999-040, Universität Hamburg, 1999.

[35] A. L. Schorlemmer, Monitoring Radiation Damage in the ATLAS Pixel Detector. $\mathrm{PhD}$ thesis, II.Physik-UniGö-Diss-2014/03, Georg-August-Universität Göttingen, 2014.

[36] H. E. Boesch et al., Saturation of Threshold Voltage Shift in MOSFET's at High Total Dose, IEEE Trans. Nucl. Sci. 33 (1986) no. 6, 1191.

[37] G. Aad et al., ATLAS pixel detector electronics and sensors, JINST 3 (2008) no. 07, P07007.

[38] ROSE Collaboration, G. Lindström, S. Watts, and F. Lemeilleur, 3rd RD48 status report: the ROSE collaboration ( $R \& D$ on silicon for future experiments), Tech. Rep. CERN-LHCC-2000-009, CERN, 1999.

[39] I. Perić et al., The FEI3 readout chip for the ATLAS pixel detector, Nucl. Instrum. Met. A 565 (2006) no. 1, 178.

[40] ATLAS Collaboration, ATLAS Insertable B-Layer Technical Design Report, Tech. Rep. CERN-LHCC-2010-013. ATLAS-TDR-19, CERN, Geneva, 2010.

[41] C. Marcelloni De Oliveira, IBL installation into the inner detector of the ATLAS Experiment side C, General Photo, 2014.

[42] C. Gößling et al., Evaluation of the breakdown behaviour of ATLAS silicon pixel sensors after partial guard-ring removal, Nucl. Instr. and Meth. A 624 (2010) no. 2, 410.

[43] IBL Collaboration, Prototype ATLAS IBL modules using the FE-I4A front-end readout chip, JINST 7 (2012) no. 11, P11010.

[44] A. Macchiolo. Private communication.

[45] J. Rieger, Comparison of Thin n- and p-type Bulk Silicon Pixel Sensors, Master's thesis, II.Physik-UniGö-MSc-2012/08, Georg-August-Universitat Göttingen, 2012.

[46] S. Terzo, Development of Radiation Hard Pixel Modules Employing Planar n-in-p Silicon Sensors with Active Edges for the ATLAS Detector at HL-LHC. PhD thesis, MPP-2015-291, TU Münich, 2015.

[47] J. Schambach et al., A MAPS Based Micro-Vertex Detector for the STAR Experiment, Phys. Procedia 66 (2015) 514. 
[48] I. Perić, A novel monolithic pixelated particle detector implemented in high-voltage CMOS technology, Nucl. Instr. and Meth. A 582 (2007) no. 3, 876.

[49] F. Hügging. Private communication.

[50] I. Perić, C. Kreidl, and P. Fischer, Hybrid pixel detector based on capacitive chip to chip signal-transmission, Nucl. Instr. and Meth. A 617 (2010) no. 1-3, 576.

[51] I. Peric, Active pixel sensors in high-voltage CMOS technologies for ATLAS, JINST 7 (2012) no. 08, C08002.

[52] M. Backhaus, High bandwidth pixel detector modules for the ATLAS Insertable B-Layer. PhD thesis, BONN-IR-2014-02, Universität Bonn, 2014.

[53] USBpix - USB based readout system for ATLAS FE-I3 and FE-I4, http://icwiki.physik. uni-bonn.de/twiki/bin/view/Systems/UsbPix. website, visited on April 6th, 2016.

[54] M. Backhaus et al., Development of a versatile and modular test system for ATLAS hybrid pixel detectors, Nucl. Inst. and Meth. A 650 (2011) no. 1, 37.

[55] J. Agricola, Development Of A Faster Test System For ATLAS Pixel Front End Electronics, Master's thesis, II.Physik-UniGo-MSc-2014/05, Georg-August-Universitat Göttingen, 2014.

[56] I. Rubinskiy, An EUDET/AIDA Pixel Beam Telescope for Detector Development, Phys. Procedia 37 (2012) 923.

[57] J. Rieger, Development of a Standardised Readout System for Active Pixel Sensors in HV/HR-CMOS Technologies for ATLAS Inner Detector Upgrades, JINST 11 (2016) no. 02, C02075.

[58] J. Behr, Jets at High $Q^{2}$ at HERA and Test Beam Measurements with the EUDET Pixel Telescope. PhD thesis, DESY-THESIS-2010-038, Universität Hamburg, 2010.

[59] T. Behnke et al., Test Beams at DESY, EUDET-Memo-2007-11 (2007) .

[60] C. Hu-Guo et al., First reticule size MAPS with digital output and integrated zero suppression for the EUDET-JRA1 beam telescope, Nucl. Instrum. Met. A 623 (2010) no. $1,480$.

[61] D. Cussans, Description of the JRA1 Trigger Logic Unit (TLU), v0.2, EUDET-Memo-2008-50 (2008) .

[62] E. Corrin, EUDAQ Software User Manual, EUDET-Memo-2010-01 (2010) .

[63] D.Haas, The EUDET High Resolution Pixel Telescope - Towards the Final Telescope, EUDET-Report-2008-02 (2008) .

[64] M. George, Testbeam Measurements with Pixel Sensors for the ATLAS Insertable b-Layer Project. PhD thesis, II.Physik-UniGö-Diss-2014/01, Georg-August-Universität Göttingen, 2014. 
[65] V. Blobel and C. Kleinwort, A New Method for the High-Precision Alignment of Track Detectors, DESY-02-077. DESY-2002-077 (2002) .

[66] R. Frühwirth and A. Strandlie, Track fitting with ambiguities and noise: A study of elastic tracking and nonlinear filters, Comput.Phys.Commun. 120 (1999) no. 2-3, 197.

[67] K. Lehmann, Implementation of the support of arbitrary pixel geometries in an existing testbeam analysis framework, Bachelor's thesis, II.Physik-UniGö-BSc-2013/04, Georg-August-Universitat Göttingen, 2013.

[68] I. Perić. Private communication.

[69] M.-M. Bé et al., Table of Radionuclides, vol. 3 of Monographie BIPM-5. Bureau International des Poids et Mesures, 2006. 



\section{Danksagung}

First of all I would like to thank everyone who helped and supported me during my time as a physics student. Without all of you this study would not have been possible. Physics is teamwork.

Ich danke Prof. Dr. Arnulf Quadt für die Möglichkeit im II. Physikalischen Institut meine Doktorarbeit anzufertigen, die Betreuung und den Blick über den Tellerand.

Vielen Dank auch an Prof. Dr. Claus Gößling, der sich bereit erklärt hat der Koreferent für diese Arbeit zu sein.

Danken möchte ich auch der Konrad-Adenauer-Stiftung, die mich während meiner Studienund Promotionszeit gefördert haben. Ohne diese Förderung wäre das Studium nicht in dieser Form möglich gewesen.

The measurements leading to these results have been performed at the Test Beam Facility at DESY Hamburg (Germany), a member of the Helmholtz Association (HGF).

I would like to thank all the current and former members of the II. Institute of Physics. It was a really nice and friendly atmosphere. It really was working with friends.

Ein besonderer Dank geht auch an Jens und Jörn für die geduldige Einführung in die faszinierende Welt der Teilchendetektoren, die Unterstützung und die Antworten auf alle Fragen.

Des Weiteren möchte ich besonders für die tollen Zeiten im Büro, Labor und beim Kickern danken: Boris, Enrico, Helge, Johannes, Knut, Konstantin, Lars, Matze, Tobias und alle anderen, die mich begleitet haben. Es wird für immer eine unvergessliche Zeit bleiben.

Für die Hilfe und Unterstützung mit dem Papierkram und anderen Verwaltungsaufgaben möchte ich den aktuellen und ehemaligen Mitarbeiterinnen des Sekretariats danken. Des Weiteren, wäre diese Arbeit ohne die schnelle und gute Hilfe der beiden Werkstätten nicht möglich gewesen, wofür ich mich bedanke.

Ein Dankeschön geht an Knut für die Erstellung dieses wunderschönen LaTeX-Layouts und die Beratung.

Teststrahlmessungen sind nicht möglich ohne Hilfe von lieben Kollegen. Besonders möchte ich Tobias für die vielen Nachtschichten und seine Unterschützung danken.

Schließlich ging es ans Schreiben der Arbeit. Ich danke euch: Boris, Carina, Jens, Johannes, Jörn und Matze, die ihr so fleißig, geduldig und konstruktiv meine Arbeit Korrektur gelesen habt. For the proofreading with focus on the English grammar I would like to thank Brendon.

Ohne einen Ausgleich zur Arbeit kommt man nicht weit. Deshalb geht auch ein Dankeschön an meine lieben Freunde, für die gemeinsamen Mittagessen, die Geburtstagsfeiern, Kochund Spieleabende und dass ihr immer für mich da seid. 
Einen ganz großer Dank geht an meine Eltern, die mich unterstützen, motivieren, mir den Rücken freihalten und immer für mich da sind. Und Alex, vielen Dank für deine Unterstützung und dass du mich immer zum Lachen bringen kannst. Ohne euch hätte ich den langen Weg nicht meistern können. 


\section{Julia Rieger}

\section{Persönliche Daten}

Name

Julia Rieger

Geburtsdatum

Staatsangehörigkeit

Familienstand

10.09.1987 in Münden

deutsch

ledig

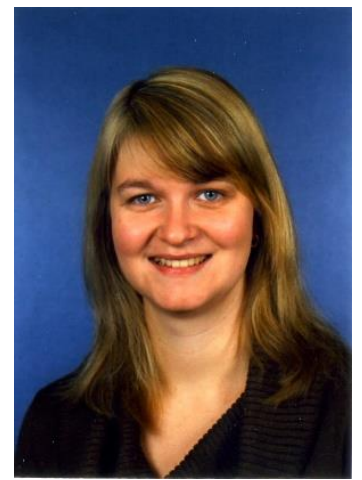

\section{Akademische Laufbahn}

Seit $01 / 2013$

$10 / 2010-10 / 2012$

$10 / 2007-08 / 2010$

$06 / 2007$

\section{Auslandsaufenthalte}

10 / $2014-12$ / 2014

$08 / 2011-10 / 2011$

$08 / 2010-01 / 2011$

\section{Praktische Erfahrung}

02 / $2014-11 / 2015$
Doktorarbeit am II. Physikalischen Institut, Georg-August-Universität Göttingen Schwerpunkt: Detektorphysik, Mikroelektronikentwicklung, Teilchenphysik Titel Detector Development for the High Luminosity Large Hardon Collider Abschluss: Dr. rer. nat. (voraussichtlich August 2016)

Physikstudium (Master) an der Georg-August-Universität Göttingen Schwerpunkt: Kern- und Teilchenphysik, Detektorphysik Masterarbeit: Comparison of Thin n-and p-type Bulk Silicon Pixel Sensors Abschluss: $\quad$ Master of Science (Note: 1,2)

Physikstudium (Bachelor) an der Georg-August-Universität Göttingen Bachelorarbeit: Measurement of Operational Stability of the ATLAS Pixel Read-Out Chip at low Threshold

Abschluss: $\quad$ Bachelor of Science (Note: 1,7)

Abitur am Grotefend - Gymnasium Münden (Note: 1,3)

Forschungsaufenthalt am Lawrence Berkeley National Laboratory, USA Thema: Entwicklung von aktiven CMOS-Sensoren

Forschungspraktikum bei der Europäische Organisation für Kernforschung, CERN Thema: Datenanalyse zum ATLAS-Pixeldetektor

ERASMUS-Semester an der Universität Stockholm, Schweden Thema: Effizienzstudien zum ATLAS-Triggersystem

Tagungsorganisation der Deutsche Physikerinnentagung 2015, Deutsche Physikalische Gesellschaft e. V.

- Koordination der Tagungsorganisation

- Finanzplanung und Kontakt zu Mitveranstaltern

- Konzeption und Organisation des Programms

04 / 2011 - 09 / 2015 Lehre an der Georg-August-Universität Göttingen

- Leitung von Übungsgruppen

- Betreuung von Bachelor- und Masterstudierenden 


\section{Stipendien}

$06 / 2013-05 / 2016$

$10 / 2007-09 / 2012$

Seit 02 / 2014

2016
Stipendium der Promotionsförderung der Konrad-Adenauer Stiftung

Stipendium der Studienförderung der Konrad-Adenauer Stiftung

- Stipendiatensprecherin der Göttinger Gruppe 2 (10/ 2009 - 10 / 2010)

- Organisation von Initiativseminaren

\section{Veröffentlichungen}

Development of a Standardised Readout System for Active Pixel Sensors in HV/HR-CMOS Technologies for ATLAS Inner Detector Upgrades [Journal of Instrumentation 11, Nr. 02, S. C02075]

Autorin der ATLAS Kollaboration (Ko-Autorin von 189 Publikationen)

\section{Kenntnisse \& Fähigkeiten}

$\begin{array}{ll}\begin{array}{l}\text { Englisch } \\ \text { Französisch } \\ \text { Schwedisch }\end{array} & \begin{array}{l}\text { Fließend } \\ \text { Grundkenntnisse } \\ \text { Grundkenntnisse }\end{array} \\ \text { Programmierung } & \text { C++ und HTML (Grundkenntnisse) } \\ \text { Präsentationserstellung } & \text { LaTeX Beamer und Microsoft Powerpoint (sehr gute Kenntnisse) } \\ \text { Textverarbeitung } & \text { LaTeX, Microsoft Word und LibreOffice Writer (sehr gute Kenntnisse) } \\ \text { Tabellenkalkulation } & \text { Microsoft Excel und LibreOffice Calc (sehr gute Kenntnisse) }\end{array}$

\section{Besonderes Engagement}

$08 / 2013-04 / 2016$

Seit 2011

$09 / 2011$

Seit $10 / 2008$
Gleichstellungsbeauftragte am II. Physikalischen Institut, Göttingen

Öffentlichkeitsarbeit im Netzwerk Teilchenwelt (Rent-a-Scientist Programm) Durchführung von Schüler-Projekttagen zum Thema Teilchenphysik

Erhalt der Niedersächsischen Ehrenamtskarte

Mitglied der Freiwilligen Feuerwehr Lutterberg

Betreuerin der Jugendfeuerwehr 